\title{
WestVirginiaUniversity
}

THE RESEARCH REPOSITORY @ WVU

Graduate Theses, Dissertations, and Problem Reports

2004

\section{Evaluation of novel cross -linking agents for gelatin/collagen matrices}

Brenda J. Schuler

West Virginia University

Follow this and additional works at: https://researchrepository.wvu.edu/etd

\section{Recommended Citation}

Schuler, Brenda J., "Evaluation of novel cross -linking agents for gelatin/collagen matrices" (2004). Graduate Theses, Dissertations, and Problem Reports. 2092.

https://researchrepository.wvu.edu/etd/2092

This Dissertation is protected by copyright and/or related rights. It has been brought to you by the The Research Repository @ WVU with permission from the rights-holder(s). You are free to use this Dissertation in any way that is permitted by the copyright and related rights legislation that applies to your use. For other uses you must obtain permission from the rights-holder(s) directly, unless additional rights are indicated by a Creative Commons license in the record and/ or on the work itself. This Dissertation has been accepted for inclusion in WVU Graduate Theses, Dissertations, and Problem Reports collection by an authorized administrator of The Research Repository @ WVU.

For more information, please contact researchrepository@mail.wvu.edu. 


\title{
Evaluation of Novel Cross-linking Agents for Gelatin/Collagen Matrices
}

\author{
Brenda J. Schuler
}

\author{
DISSERTATION \\ Submitted to the School of Pharmacy \\ at West Virginia University
}

In partial fulfillment of the requirements

for the degree of

\section{Doctor of Philosophy}

in

Pharmaceutics

\author{
Paula Jo Meyer-Stout, Ph.D., Chair \\ William J. Addicks, Ph.D. \\ Michael Bagby, DDS, Ph.D. \\ Yongyut Rojanasakul, Ph.D. \\ Timothy S. Tracy, Ph.D. \\ Department of Basic Pharmaceutical Sciences \\ Morgantown, West Virginia
}

Keywords: gelatin, sucrose, fructose, localized drug delivery

Copyright 2004 Brenda J. Schuler 


\section{ABSTRACT \\ Evaluation of Novel Cross-linking Agents for Gelatin/Collagen Matrices Brenda J. Schuler}

Useful for drug delivery systems, natural polymer matrix systems, such as gelatin, have been formulated for localized drug delivery. The main limitations of gelatin are its extensive swelling, rapid dissolution and drug release. To slow drug release, chemical cross-linking agents have been used to form relatively non-soluble networks. Sucrose and fructose were used as cross-linking agents in a gelatin-based matrix. Additional studies evaluated two process variables: casting and curing (ambient or refrigerated temperatures, vacuum drying and lyophilization) methods. The optimized gelatin/sugar matrices were then compared to varying concentrations of gelatin/formaldehyde matrices. Matrix erosion studies were completed to monitor percent swelling and maintenance of physical integrity. Thermal analysis studies were performed to thermally characterize the matrices. Optimized formulations were loaded with drug and the release profiles of these matrices were characterized and compared. Qualitative and quantitative studies were completed, under sink and non-sink conditions. Diffusion based models were applied for identification of the mechanisms controlling drug release from these matrices.

The use of native sugars as cross-linking agents enhances matrix performance and overall stability while decreasing the risks associated with aldehydes. It was determined that lyophilization provided optimum matrix curing. The use of ethanol as a dispersing agent offered little advantage over water. Comparison of gelatin/sugar and gelatin/formaldehyde matrices demonstrated that the sugars decrease the swelling of the matrix, but do not maintain the prolonged erosion time seen with the gelatin/formaldehyde matrices. Visual release studies revealed that an initial burst followed by a gradual release is seen until there is no dye in the matrix. Visual studies also demonstrated that two different solutes transport out of the matrices at different rates. In vitro release and modeling studies revealed that the dye release appears to be diffusion-controlled, with minimal affect of swelling on release kinetics.

These studies demonstrate the viability of the use of sucrose and fructose, in combination with lyophilization, as cross-linking agents for gelatin-based systems. While these sugars could not replace formaldehyde in formulations needed for extended sustained release delivery ( $>2$ day), the sugars offer a better alternative in formulations requiring an extended release of drug over 12-48 hours. 


\section{Dedication}

This work is dedicated to my parents

Barry and Kathy Schuler 


\section{ACKNOWLEDGEMENTS}

It gives me great pleasure to acknowledge the support of every individual who has contributed to this dissertation. First, I wish to thank my research advisor, Dr. Paula Jo Stout, for her advice and expert opinion on the dissertation project. I greatly appreciate her guidance throughout the course of this work. Her patience and understanding have been invaluable during my years in graduate school.

I would like to extend my sincerest appreciation to the other members of my advisory committee: Dr. William J Addicks, Dr. Michael Bagby, Dr. Yongyut Rojanasakul, and Dr. Timothy Tracy, for their timely input and helpful advice on the research project.

A special thanks goes out to Dr. James Mahaney for the many hours of help with the lyophilization portion of this research. I would also like to thank Dr. Terry Gullion for all of his help and expertise on solid-state NMR.

I wish to thank my parents for their unconditional love and support, this has been the constant source of my strength. They deserve all of the credit for my achievements. Thank you to my sister, Jamie, and brother, Michael, for always being there for me. I would also like to thank Jamie for the countless hours she spent proof reading my reports.

I would like to thank Charles for all of his patience and understanding. He has been a constant source of inspiration and his support has enabled me to reach my goals. I cannot thank him enough for the countless hours he spent helping me and for always being there for me.

I extend my thanks to the entire faculty, staff, and graduate students in the School of Pharmacy for their help and advice. I wish to thank Penny Dailey for all of her support. I would also like to thank Tina Bland for all of her support, ideas, and suggestions. 


\section{TABLE OF CONTENTS}

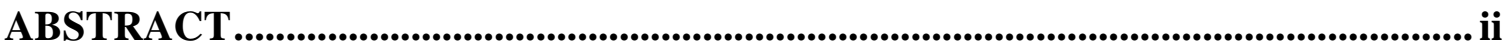

ACKNOWLEDGEMENTS .............................................................................................. iv

LIST OF TABLES .................................................................................................................. viii

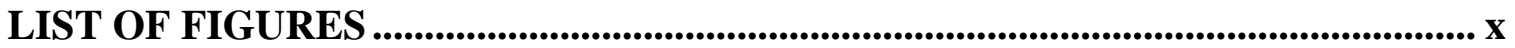

LIST OF APPENDICES .................................................................................................xviii

Chapter I..................................................................................................................................... 1

INTRODUCTION AND LITERATURE REVIEW .......................................................... 1

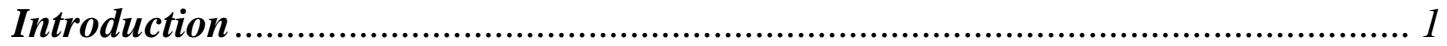

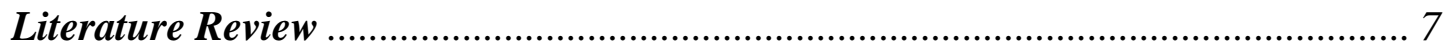

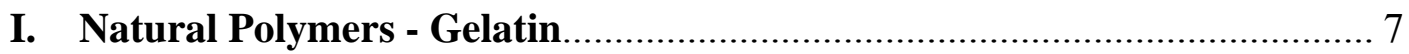

II. Gelatin Matrix - Formation and Behavior............................................ 11

III. Gelatin Matrix - Delivery Applications ………….............................. 22

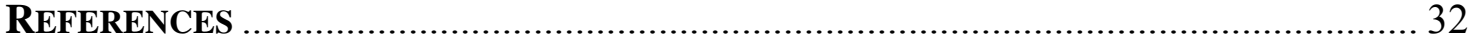

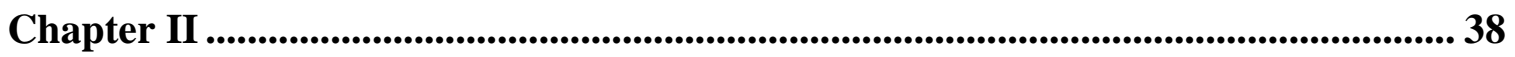

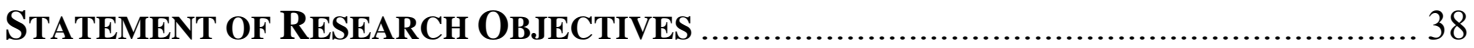

Chapter III.................................................................................................................................. 40

FORMULATION DEVELOPMENT AND OPTIMIZATION …………................................ 40

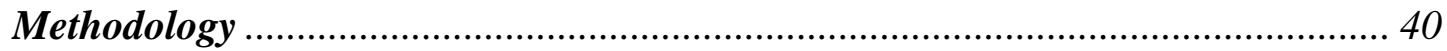

I. Evaluation of Cross-linking Agent - Sugars ……………………............... 41

II. Evaluation of the Efficacy of the Sugars............................................... 41

III. Experimental Design for Cross-linking Optimization.............................. 44

IV. Evaluation of Excipients - Dispersion Agent …………………............. 45

V. Evaluation of the Efficacy of the Dispersion Agent ................................. 46

VI. Experimental Design for Excipient Optimization...................................... 46

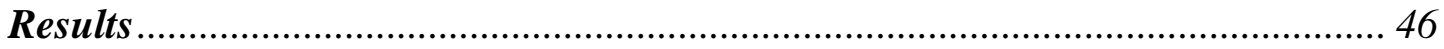

I. Visual Observations .............................................................................. 47

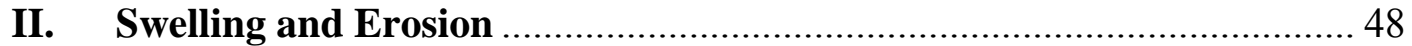

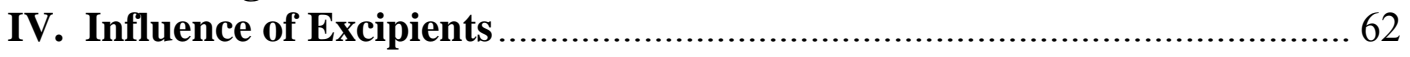

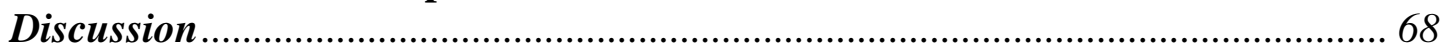

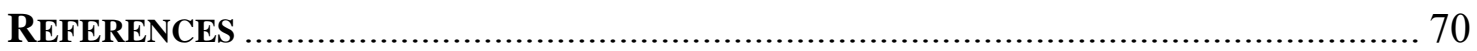


Chapter IV .................................................................................................................... 71

PROCESS DESIGN AND OPTIMIZATION …………….............................................. 71

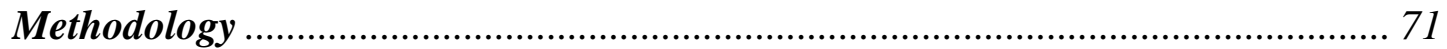

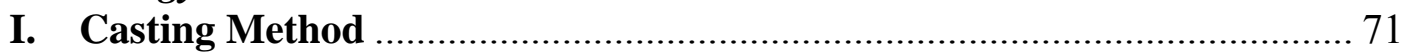

II. Curing Method ................................................................................. 72

III. Experimental Design for Manufacturing Optimization Studies ............ 74

Results ................................................................................................... 75

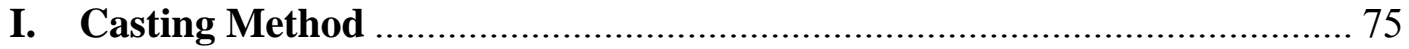

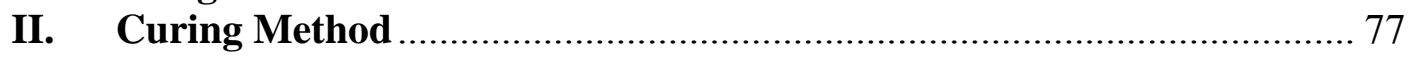

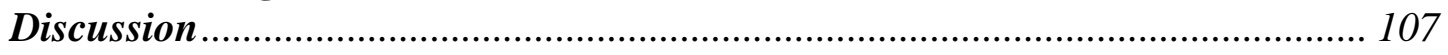

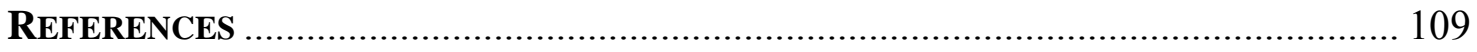

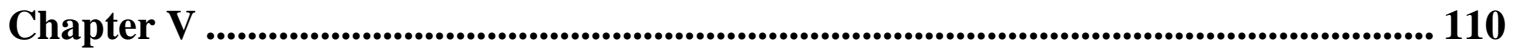

COMPARISON OF OPTIMIZED NOVEL GELATIN/SUGAR MATRICES WITH

TRADITIONAL GELATIN/FORMALDEHYDE MATRICES ........................................... 110

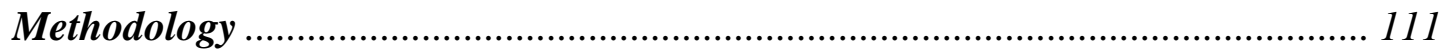

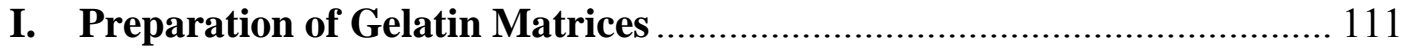

II. Evaluation of swelling and bioerosion ........................................... 112

III. Experimental Design for Cross-linking Agent Comparison Studies.... 113

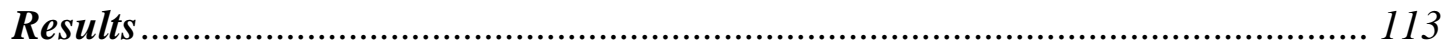

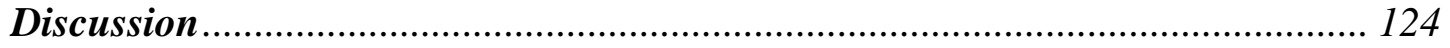

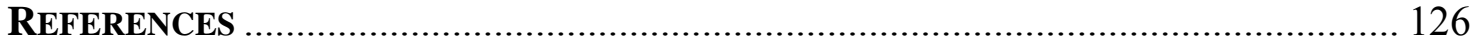

Chapter VI....................................................................................................................... 127

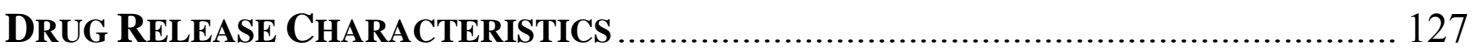

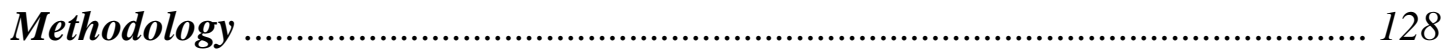

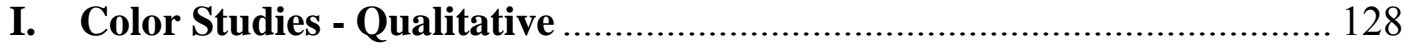

II. Drug Loading Studies - Quantitative …………….............................. 133

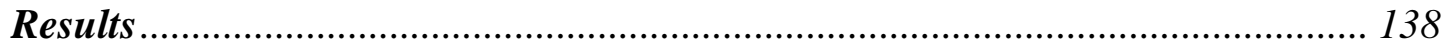

I. Content Analysis of the Matrices........................................................ 138

II. Dye Studies - Qualitative Visual ........................................................ 139

III. Dye Studies - Quantitative Release ..................................................... 149

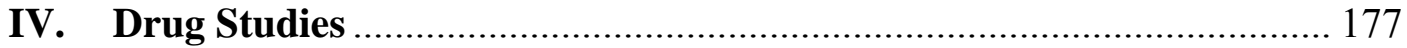

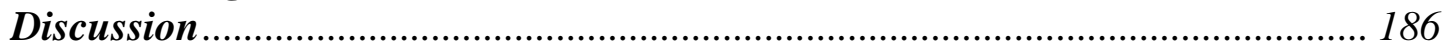

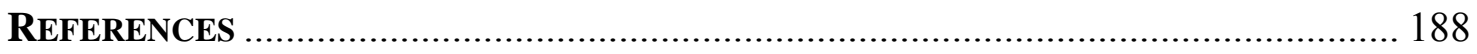


Chapter VII ............................................................................................................................ 189

DELIVERY SYSTEM MODELING ............................................................................ 189

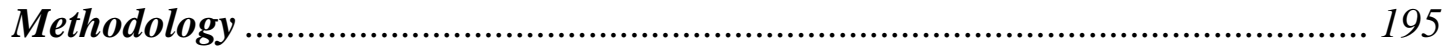

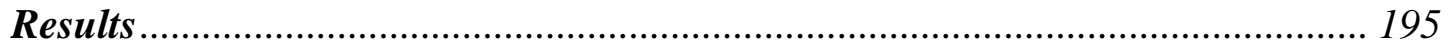

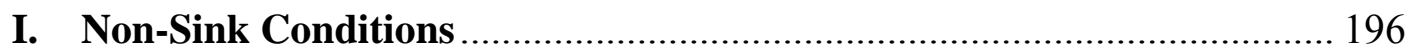

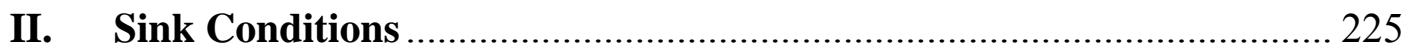

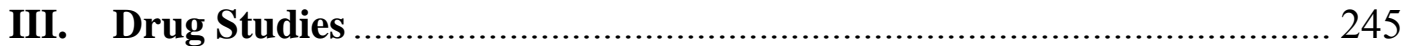

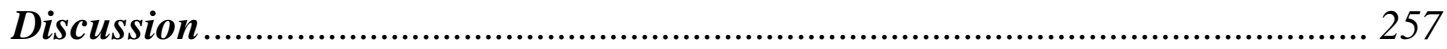

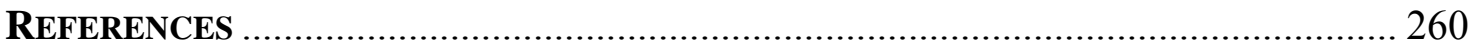

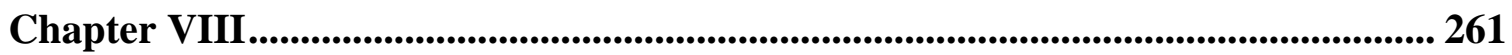

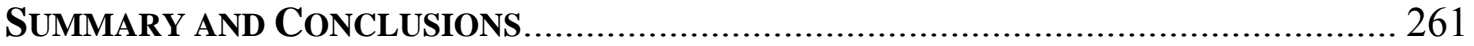

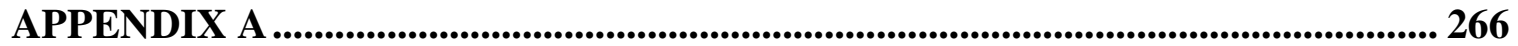

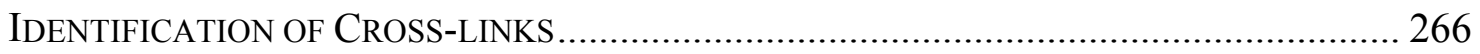

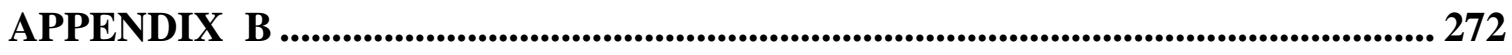

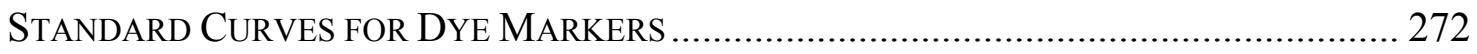

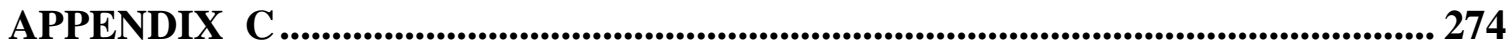

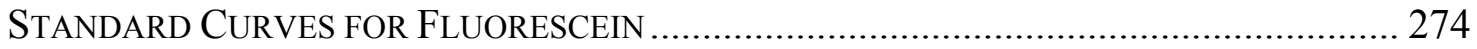




\section{LIST OF TABLES}

TABLE 1. A COMPARISON OF THE INITIAL WEIGHTS FOR THE GELATIN, GELATIN/30\% SUCROSE AND GELATIN/15.8\% FRUCTOSE MATRICES. .......................................... 50

TABLE 2. COMPARISON OF THE VARIABILITY OF SWELLING PROFILES............................. 52

TABLE 3. COMPARISON OF $\mathrm{T}_{\mathrm{M}}$ AND PEAK AREA OF THE GELATIN/WATER AND GELATIN/70\% ETHANOL/30\% WATER FORMULATIONS BEFORE AND AFTER VACUUM DRYING FOR 30 MINUTES.

TABLE 4. COMPARISON OF THE PERCENT WATER LOSS ( \pm SD) FOR THE THREE FORMULATIONS AFTER THE VACUUM DRYING PROCESS WAS APPLIED FOR 30 MINUTES OVER A 7 DAY REFRIGERATION PERIOD.

TABLE 5. COMPARISON OF WATER LOST AFTER SAMPLES WERE REFRIGERATED FOR 24 HOURS FOLLOWED BY EITHER 24 OR 36 HOURS OF LYOPHILIZATION.

TABLE 6. COMPARISON OF PROCESS EFFECTS OF THE OPTIMIZED FORMULATIONS 105

TABLE 7. COMPARISON OF $\mathrm{T}_{\mathrm{M}}$ AMONG THE FIVE FORMULATIONS.

TABLE 8. COMPARISON OF $\mathrm{T}_{\mathrm{M}}$ AND $\%$ WEIGHT LOSS OF MATRICES WITH AND WITHOUT DYE. 176

TABLE 9. COMPARISON OF THE VARIABILITY OF FLUORESCEIN RELEASE.

TABLE 10. CORRELATION COEFFICIENTS OF DIFFERENT MATHEMATICAL MODELS FOR GREEN DYE.

TABLE 11. CORRELATION COEFFICIENTS OF DIFFERENT MATHEMATICAL MODELS FOR RED DYE.

TABLE 12. COMPARISON OF THE REGRESSION DATA FOR THREE DIFFUSIONAL PHASES OF THE GREEN DYE.

TABLE 13. COMPARISON OF THE REGRESSION DATA FOR THREE DIFFUSIONAL PHASES OF THE RED DYE.

TABLE 14. CORRELATION COEFFICIENTS OF DIFFERENT MATHEMATICAL MODELS FOR GREEN DYE.

TABLE 15. CORRELATION COEFFICIENTS OF DIFFERENT MATHEMATICAL MODELS FOR RED DYE. 
TABLE 16. COMPARISON OF THE REGRESSION DATA FOR THREE DIFFUSIONAL PHASES OF

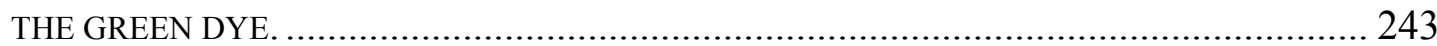

TABLE 17. COMPARISON OF THE REGRESSION DATA FOR THREE DIFFUSIONAL PHASES OF THE RED DYE.

TABLE 18. CORRELATION COEFFICIENTS AND LINEAR REGRESSION DATA FOR FLUORESCEIN

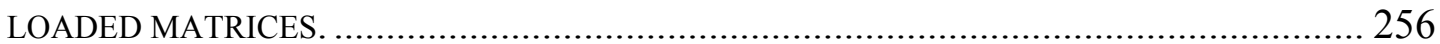




\section{LIST OF FIGURES}

Figure 1. PRoteIn BACKBONE OF GELATIN WHERE R IS ANY AMINO ACID. ...................... 10

FIGURE 2. FISHNET STRUCTURE OF GELATIN WITH STRONG POINTS SEPARATED BY FLEXIBLE

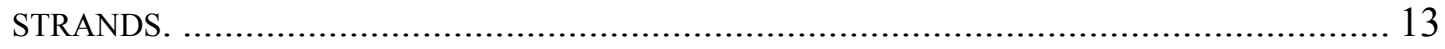

FIGURE 3. INTER- AND INTRASTRAND CROSS-LINKING WITHIN THE GELATIN. ................... 14

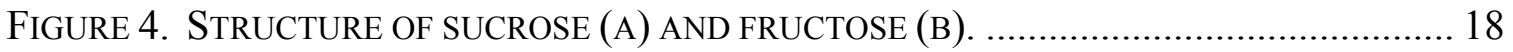

FIGURE 5. SCHEMATIC REPRESENTATION OF THE CROSS-LINKING REACTION MECHANISM BETWEEN THE SUGARS AND GELATIN. ............................................................... 20

FIGURE 6. SWELLING AND EROSION PROFILES COMPARING GELATIN AND EQUAL MOLAR GELATIN/30\% SUCROSE, AND GELATIN/15.8\% FRUCTOSE MATRICES. ........................ 49

FIGURE 7. SWELLING AND EROSION PROFILES COMPARING EQUAL CONCENTRATIONS OF GELATIN/30\% SUCROSE AND GELATIN/30\% FRUCTOSE MATRICES. ........................... 53

FIGURE 8. SWELLING AND EROSION PROFILE OF VARYING CONCENTRATIONS OF SUCROSE IN

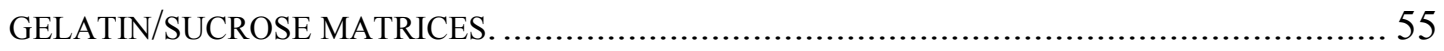

FIGURE 9. SWELLING AND EROSION PROFILE OF VARYING CONCENTRATIONS OF FRUCTOSE

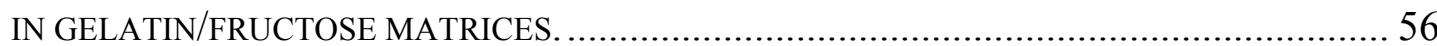

Figure 10. THERMAL ANALYSIS SCANS OF THE GELATIN (A), GELATIN/30\% SUCROSE (B), AND GELATIN/15.8\% FRUCTOSE (C) MATRICES........................................................ 58

FIGURE 11. THERMAL ANALYSIS SCAN OF THE MELTING TEMPERATURE OF SUCROSE. ...... 60

FIGURE 12. THERMAL ANALYSIS SCAN OF THE MELTING TEMPERATURE OF FRUCTOSE. .... 61

FIGURE 13. SWELLING AND EROSION PROFILES OF WATER-BASED GELATIN AND ETHANOL-

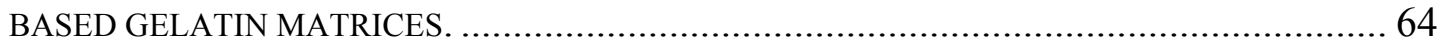

FigurE 14. THERMAL ANALYSIS SCANS OF ETHANOL-BASED GELATIN (A), GELATIN/30\% SUCROSE (B), AND GELATIN/15.8\% FRUCTOSE (C) MATRICES. ................................. 65

FIGURE 15. SWELLING AND EROSION PROFILE OF GELATIN MATRICES OVER A 7 DAY

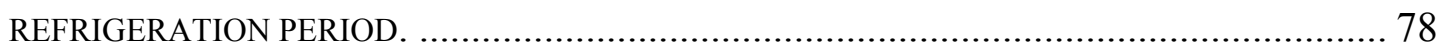

FIGURE 16. SWELLING AND EROSION PROFILE OF GELATIN/30\% SUCROSE MATRICES OVER A 7 DAY REFRIGERATION PERIOD. 
FIGURE 17. SWELLING AND EROSION PROFILE OF GELATIN/15.8\% FRUCTOSE MATRICES

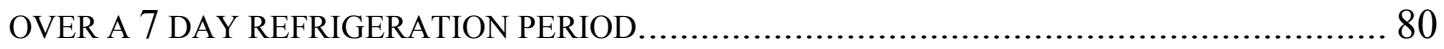

FigURE 18. SWELLING AND EROSION PROFILE OF GELATIN, GELATIN/30\% SUCROSE, AND GELATIN/15.8\% FRUCTOSE MATRICES REFRIGERATED FOR 7 DAYS (NON-CROSS-LINKED

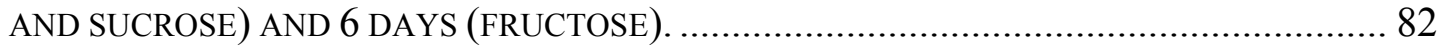

FIGURE 19. THERMAL ANALYSIS SCANS OF THE GELATIN MATRIX. .................................. 83

FIGURE 20. THERMAL ANALYSIS SCANS OF THE GELATIN/30\% SUCROSE MATRIX.............. 84

FigURE 21. THERMAL ANALYSIS SCANS OF THE GELATIN/15.8\% FRUCTOSE MATRIX........ 85

FigURE 22. THERMAL ANALYSIS SCANS OF A GELATIN MATRIX BEFORE (A) AND AFTER (B)

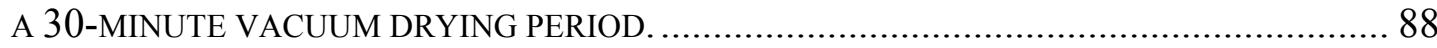

FigURE 23. THERMAL ANALYSIS SCANS OF A GELATIN/30\% SUCROSE MATRIX BEFORE (A) AND AFTER (B) A 30-MINUTE VACUUM DRYING PERIOD.

FigURE 24. THERMAL ANALYSIS OF A GELATIN/15.8\% FRUCTOSE MATRIX BEFORE (A) AND AFTER (B) A 30-MINUTE VACUUM DRYING PERIOD. 90

FIGURE 25. SWELLING AND EROSION PROFILE OF GELATIN MATRICES REFRIGERATED, R, FOR 2, 24, OR 48 HOURS FOLLOWED BY 24 HOURS OF LYOPHILIZATION, L.................... 93

FigURE 26. SWELLING AND EROSION PROFILE OF GELATIN/30\% SUCROSE MATRICES REFRIGERATED, R, FOR 2, 24, OR 48 HOURS FOLLOWED BY 24 HOURS OF LYOPHILIZATION, L

FIGURE 27. SWELLING AND EROSION PROFILE OF GELATIN/15.8\% FRUCTOSE MATRICES REFRIGERATED, R, FOR 2, 24, OR 48 HOURS FOLLOWED BY 24 HOURS OF LYOPHILIZATION, L.

FIGURE 28. SWELLING AND EROSION PROFILE OF GELATIN MATRICES REFRIGERATED, R, FOR 24 HOURS AND LYOPHILIZED, L, FOR EITHER 24 OR 36 HOURS.

FigURE 29. SWELLING AND EROSION PROFILE OF GELATIN $/ 30 \%$ SUCROSE MATRICES REFRIGERATED FOR 24 HOURS AND LYOPHILIZED FOR EITHER 24 OR 36 HOURS.

FigURE 30. SWELLING AND EROSION PROFILE OF GELATIN/15.8\% FRUCTOSE MATRICES REFRIGERATED FOR 24 HOURS AND LYOPHILIZED FOR EITHER 24 OR 36 HOURS.

FigURE 31. SWELLING AND EROSION PROFILES OF GELATIN MATRICES REFRIGERATED AND LYOPHILIZED FOR SHORT, MEDIUM, AND LONG CURING TIMES. 101 
FigURE 32. SWELLING AND EROSION PROFILES OF GELATIN/30\% SUCROSE MATRICES REFRIGERATED AND LYOPHILIZED FOR SHORT, MEDIUM, AND LONG CURING TIMES. . 102

FIGURE 33. SWELLING AND EROSION PROFILES OF GELATIN/15.8\% FRUCTOSE MATRICES REFRIGERATED AND LYOPHILIZED FOR SHORT, MEDIUM, AND LONG CURING TIMES. . 103

FIGURE 34. SWELLING AND EROSION PROFILE FOR EQUAL WEIGHT SAMPLES PRE- AND POSTLYOPHILIZATION.

FigURE 35. SWELLING AND EROSION PROFILE OF GELATIN/0.074\% FORMALDEHYDE MATRICES OVER A 7 DAY REFRIGERATION PERIOD.

FiguRE 36. SWELLING AND EROSION PROFILE OF GELATIN/0.148\% FORMALDEHYDE MATRICES OVER A 7 DAY REFRIGERATION PERIOD.

FIGURE 37. SWELLING AND EROSION PROFILE OF VARYING CONCENTRATIONS OF FORMALDEHYDE IN GELATIN/FORMALDEHYDE MATRICES.

FigURE 38. THERMAL ANALYSIS SCANS OF THE GELATIN/0.037\% FORMALDEHYDE MATRIX. 120

FigURE 39. SWELLING AND EROSION PROFILE OF GELATIN, GELATIN/SUCROSE, GELATIN/FRUCTOSE, AND GELATIN/FORMALDEHYDE.

Figure 40. Structures of Red Dye: Allura Red AC AND ERythrosine.

Figure 41. Structures of GReEn dye: Brilliant Blue FCF AND TARTRAZINE....... 130

FigURE 42. STRUCTURE OF FLUORESCEIN. 134

Figure 43. OVERVIEW OF Visual STUdies OF Gelatin (G), GELATIN/SUCROSE (S), GELATIN/FRUCTOSE (F), GELATIN/FORMALDEHYDE (H) MATRICES LOADED WITH GREEN OR RED DYE.

FigURE 44. VISUAL STUDIES OF GELATIN AND GELATIN/SUCROSE MATRICES LOADED WITH $10 \%$ GREEN DYE.

FIGURE 45. VISUAL STUDIES OF GELATIN AND GELATIN/SUCROSE MATRICES LOADED WITH $10 \%$ RED DYE. 144

FigURE 46. VISUAL STUDIES OF GELATIN AND GELATIN/SUCROSE MATRICES LOADED WITH $10 \%$ GREEN AND $10 \%$ RED DYE.

FIGURE 47. RELEASE PROFILE OF NON-CROSS-LINKED GELATIN MATRICES LOADED WITH RED, GREEN, OR A COMBINATION OF RED \& GREEN DYE. 150 
FIGURE 48. RELEASE PROFILE OF GELATIN/30\% SUCROSE MATRICES LOADED WITH RED, GREEN, OR A COMBINATION OF RED \& GREEN DYE. ............................................. 152

FIGURE 49. RELEASE PROFILE OF GELATIN/15.8\% FRUCTOSE MATRICES LOADED WITH RED, GREEN, OR A COMBINATION OF RED \& GREEN DYE. 153

FIGURE 50. RELEASE PROFILE OF GELATIN/0.037\% FORMALDEHYDE MATRICES LOADED WITH RED, GREEN, OR A COMBINATION OF RED \& GREEN DYE.

FIGURE 51. COMPARISON OF THE RELEASE PROFILE TO THE SWELLING/EROSION PROFILE OF GELATIN MATRICES. 158

FIGURE 52. COMPARISON OF THE RELEASE PROFILE TO THE SWELLING/EROSION PROFILE OF GELATIN $/ 30 \%$ SUCROSE MATRICES. 159

FIGURE 53. COMPARISON OF THE RELEASE PROFILE TO THE SWELLING/EROSION PROFILE OF GELATIN/15.8\% FRUCTOSE MATRICES. 160

FIGURE 54. COMPARISON OF THE RELEASE PROFILE TO THE SWELLING/EROSION PROFILE OF GELATIN $/ 0.037 \%$ FORMALDEHYDE MATRICES. 161

FIGURE 55. RELEASE PROFILE OF NON-CROSS-LINKED GELATIN MATRICES LOADED WITH RED, GREEN, OR A COMBINATION OF RED \& GREEN DYE.

FIGURE 56. RELEASE PROFILE OF NON-CROSS-LINKED GELATIN/30\% SUCROSE MATRICES LOADED WITH RED, GREEN, OR A COMBINATION OF RED \& GREEN DYE. 165

FIGURE 57. RELEASE PROFILE OF NON-CROSS-LINKED GELATIN/15.8\% FRUCTOSE MATRICES LOADED WITH RED, GREEN, OR A COMBINATION OF RED \& GREEN DYE. ... 166

FIGURE 58. RELEASE PROFILE OF NON-CROSS-LINKED GELATIN/0.037\% FORMALDEHYDE MATRICES LOADED WITH RED, GREEN, OR A COMBINATION OF RED \& GREEN DYE. ... 168

FIGURE 59. COMPARISON OF THE RELEASE PROFILE TO THE SWELLING/EROSION PROFILE FOR GELATIN MATRICES.

FIGURE 60. COMPARISON OF THE RELEASE PROFILE TO THE SWELLING/EROSION PROFILE FOR GELATIN $/ 30 \%$ SUCROSE MATRICES.

FIGURE 61. COMPARISON OF THE RELEASE PROFILE TO THE SWELLING/EROSION PROFILE FOR GELATIN/15.8\% FRUCTOSE MATRICES.

FIGURE 62. COMPARISON OF THE RELEASE PROFILE TO THE SWELLING/EROSION PROFILE FOR GELATIN/0.037\% FORMALDEHYDE MATRICES. 
FIGURE 63. RELEASE PROFILES OF GELATIN, GELATIN/30\% SUCROSE, GELATIN/ $15.8 \%$ FRUCTOSE AND GELATIN/0.037\% FORMALDEHYDE MATRICES LOADED WITH 0.1\% FLUORESCEIN SODIUM.

FIGURE 64. COMPARISON OF THE RELEASE PROFILE TO THE SWELLING/EROSION PROFILE FOR GELATIN MATRICES LOADED WITH FLUORESCEIN.

FIGURE 65. COMPARISON OF THE RELEASE PROFILE TO THE SWELLING/EROSION PROFILE FOR GELATIN $/ 30 \%$ SUCROSE MATRICES LOADED WITH FLUORESCEIN.

FIGURE 66. COMPARISON OF THE RELEASE PROFILE TO THE SWELLING/EROSION PROFILE FOR GELATIN/15.8\% FRUCTOSE MATRICES LOADED WITH FLUORESCEIN.

FIGURE 67. COMPARISON OF THE RELEASE PROFILE TO THE SWELLING/EROSION PROFILE FOR GELATIN/0.037\% FORMALDEHYDE MATRICES LOADED WITH FLUORESCEIN....... 184

FIGURE 68. RELEASE PROFILE OF GELATIN, GELATIN/30\% SUCROSE, GELATIN/15.8\% FRUCTOSE, AND GELATIN/0.037\% FORMALDEHYDE MATRICES LOADED WITH GREEN DYE.

FIGURE 69. RELEASE PROFILE OF GELATIN, GELATIN/30\% SUCROSE, GELATIN/15.8\% FRUCTOSE, AND GELATIN/0.037\% FORMALDEHYDE MATRICES LOADED WITH RED DYE.

FIGURE 70. LINEAR REGRESSION FOR THE FIRST ORDER EQUATION OVER THE SECOND RELEASE PHASE (HOURS 4-12) FOR MATRICES LOADED WITH GREEN DYE.

FIGURE 71. LINEAR REGRESSION FOR THE FIRST ORDER EQUATION OVER THE THIRD RELEASE PHASE (HOURS 24-64) FOR MATRICES LOADED WITH GREEN DYE.

FIGURE 72. LINEAR REGRESSION FOR THE HIGUCHI SQUARE ROOT EQUATION OVER THE SECOND RELEASE PHASE (HOURS 4-12) FOR MATRICES LOADED WITH GREEN DYE.... 204

FIGURE 73. LINEAR REGRESSION FOR THE HIGUCHI SQUARE ROOT EQUATION OVER THE THIRD RELEASE PHASE (HOURS 24-64) FOR MATRICES LOADED WITH GREEN DYE..... 205

FIGURE 74. LINEAR REGRESSION FOR THE HIXSON-CROWELL CUBE ROOT EQUATION OVER THE SECOND RELEASE PHASE (HOURS 4-12) FOR MATRICES LOADED WITH GREEN DYE.

FIGURE 75. LINEAR REGRESSION FOR THE HIXSON-CROWELL CUBE ROOT EQUATION OVER THE THIRD RELEASE PHASE (HOURS 24-64) FOR MATRICES LOADED WITH GREEN DYE.

FIGURE 76. LINEAR REGRESSION FOR THE FIRST ORDER EQUATION OVER THE SECOND RELEASE PHASE (HOURS 4-12) FOR MATRICES LOADED WITH RED DYE. 
FIGURE 77. LINEAR REGRESSION FOR THE FIRST ORDER EQUATION OVER THE THIRD RELEASE PHASE (HOURS 24-64) FOR MATRICES LOADED WITH RED DYE.

FIGURE 78. LINEAR REGRESSION FOR THE HIGUCHI SQUARE ROOT EQUATION OVER THE SECOND RELEASE PHASE (HOURS 4-12) FOR MATRICES LOADED WITH RED DYE........ 210

FIGURE 79. LINEAR REGRESSION FOR THE HIGUCHI SQUARE ROOT EQUATION OVER THE THIRD RELEASE PHASE (HOURS 24-64) FOR MATRICES LOADED WITH RED DYE......... 211

FigURE 80. LINEAR REGRESSION FOR THE HIXSON-CROWELL CUBE ROOT EQUATION OVER THE SECOND RELEASE PHASE (HOURS 4-12) FOR MATRICES LOADED WITH RED DYE. 212

FIGURE 81. LINEAR REGRESSION FOR THE HIXSON-CROWELL CUBE ROOT EQUATION OVER THE THIRD RELEASE PHASE (HOURS 12-64) FOR MATRICES LOADED WITH RED DYE. . 213

Figure 82. Plot OF $\mathrm{D}\left(\mathrm{M}_{\mathrm{T}} / \mathrm{M}_{\infty}\right)$ Versus $1 /\left(\mathrm{M}_{\mathrm{T}} / \mathrm{M}_{\infty}\right)$ FOR THE Gelatin MATRICES LOADED WITH GREEN DYE.

Figure 83 . Plot OF $\mathrm{D}\left(\mathrm{M}_{\mathrm{T}} / \mathrm{M}_{\infty}\right)$ VERSUS $1 /\left(\mathrm{M}_{\mathrm{T}} / \mathrm{M}_{\infty}\right)$ FOR THE GELATIN $/ 30 \%$ SUCROSE MATRICES LOADED WITH GREEN DYE.

Figure 84 . Plot OF $\mathrm{D}\left(\mathrm{M}_{\mathrm{T}} / \mathrm{M}_{\infty}\right)$ Versus $1 /\left(\mathrm{M}_{\mathrm{T}} / \mathrm{M}_{\infty}\right)$ FOR THE GELATIN $/ 15.8 \%$ FRUCTOSE MATRICES LOADED WITH GREEN DYE.

Figure 85 . Plot Of $\mathrm{D}\left(\mathrm{M}_{\mathrm{T}} / \mathrm{M}_{\infty}\right)$ Versus $1 /\left(\mathrm{M}_{\mathrm{T}} / \mathrm{M}_{\infty}\right)$ FOR THE GELATIN $/ 0.037 \%$ FORMALDEHYDE MATRICES LOADED WITH GREEN DYE.

Figure 86 . Plot OF $\mathrm{D}\left(\mathrm{M}_{\mathrm{T}} / \mathrm{M}_{\infty}\right)$ Versus $1 /\left(\mathrm{M}_{\mathrm{T}} / \mathrm{M}_{\infty}\right)$ FOR THE Gelatin MATRICES LOADED WITH RED DYE.

Figure 87. PLOT OF $\mathrm{D}\left(\mathrm{M}_{\mathrm{T}} / \mathrm{M}_{\infty}\right)$ VERSUS $1 /\left(\mathrm{M}_{\mathrm{T}} / \mathrm{M}_{\infty}\right)$ FOR THE GELATIN $/ 30 \%$ SUCROSE MATRICES LOADED WITH RED DYE.

Figure 88 . Plot OF $\mathrm{D}\left(\mathrm{M}_{\mathrm{T}} / \mathrm{M}_{\infty}\right)$ VERSUS $1 /\left(\mathrm{M}_{\mathrm{T}} / \mathrm{M}_{\infty}\right)$ FOR THE GELATIN $/ 15.8 \%$ FRUCTOSE MATRICES LOADED WITH RED DYE.

Figure 89 . Plot OF $D\left(\mathrm{M}_{\mathrm{T}} / \mathrm{M}_{\infty}\right)$ VERSUS $1 /\left(\mathrm{M}_{\mathrm{T}} / \mathrm{M}_{\infty}\right)$ FOR THE GELATIN $/ 0.037 \%$ FORMALDEHYDE MATRICES LOADED WITH RED DYE

FigURE 90. RELEASE PROFILE OF GELATIN, GELATIN/30\% SUCROSE, GELATIN $/ 15.8 \%$ FRUCTOSE, AND GELATIN $/ 0.037 \%$ FORMALDEHYDE MATRICES LOADED WITH GREEN DYE.

FigURE 91. RELEASE PROFILE OF GELATIN, GELATIN/30\% SUCROSE, GELATIN/15.8\% FRUCTOSE, AND GELATIN/0.037\% FORMALDEHYDE MATRICES LOADED WITH RED DYE. 
FIGURE 92. LINEAR REGRESSION FOR THE FIRST ORDER EQUATION OVER THE SECOND RELEASE PHASE (HOURS 12-32) FOR MATRICES LOADED WITH GREEN DYE.

FIGURE 93. LINEAR REGRESSION FOR THE FIRST ORDER EQUATION OVER THE THIRD RELEASE PHASE (HOURS 36-52) FOR MATRICES LOADED WITH GREEN DYE.

FIGURE 94. LINEAR REGRESSION FOR THE HIGUCHI SQUARE ROOT EQUATION OVER THE SECOND RELEASE PHASE (HOURS 12-32) FOR MATRICES LOADED WITH GREEN DYE. 232

FIGURE 95. LINEAR REGRESSION FOR THE HIGUCHI SQUARE ROOT EQUATION OVER THE THIRD RELEASE PHASE (HOURS 36-52) FOR MATRICES LOADED WITH GREEN DYE..... 233

FIGURE 96. LINEAR REGRESSION FOR THE HIXSON-CROWELL CUBE ROOT EQUATION OVER THE SECOND RELEASE PHASE (HOURS 12-32) FOR MATRICES LOADED WITH GREEN DYE.

FIGURE 97. LINEAR REGRESSION FOR THE HIXSON-CROWELL CUBE ROOT EQUATION OVER THE THIRD RELEASE PHASE (HOURS 36-52) FOR MATRICES LOADED WITH GREEN DYE.

FIGURE 98. LINEAR REGRESSION FOR THE FIRST ORDER EQUATION OVER THE SECOND RELEASE PHASE (HOURS 16-24) FOR MATRICES LOADED WITH RED DYE.

FIGURE 99. LINEAR REGRESSION FOR THE FIRST ORDER EQUATION OVER THE THIRD RELEASE PHASE (HOURS 28-72) FOR MATRICES LOADED WITH RED DYE.

FIGURE 100. LINEAR REGRESSION FOR THE HIGUCHI SQUARE ROOT EQUATION OVER THE SECOND RELEASE PHASE (HOURS 16-24) FOR MATRICES LOADED WITH RED DYE...... 238

FIGURE 101. LINEAR REGRESSION FOR THE HIGUCHI SQUARE ROOT EQUATION OVER THE THIRD RELEASE PHASE (HOURS 28-72) FOR MATRICES LOADED WITH RED DYE 239

FIGURE 102. LINEAR REGRESSION FOR THE HIXSON-CROWELL CUBE ROOT EQUATION OVER THE SECOND RELEASE PHASE (HOURS 16-24) FOR MATRICES LOADED WITH RED DYE.

FIGURE 103. LINEAR REGRESSION FOR THE HIXSON-CROWELL CUBE ROOT EQUATION OVER THE THIRD RELEASE PHASE (HOURS 28-72) FOR MATRICES LOADED WITH RED DYE. . 241

Figure 104. Plot OF D( $\left(\mathrm{M}_{\mathrm{T}} / \mathrm{M}_{\infty}\right)$ Versus $1 /\left(\mathrm{M}_{\mathrm{T}} / \mathrm{M}_{\infty}\right)$ FOR THE GeLATIN MATRICES LOADED WITH GREEN DYE.

Figure 105. Plot OF $\mathrm{D}\left(\mathrm{M}_{\mathrm{T}} / \mathrm{M}_{\infty}\right)$ VERSUS $1 /\left(\mathrm{M}_{\mathrm{T}} / \mathrm{M}_{\infty}\right)$ FOR THE GELATIN $/ 30 \%$ SUCROSE MATRICES LOADED WITH GREEN DYE. 
Figure 106. Plot of $\mathrm{D}\left(\mathrm{M}_{\mathrm{T}} / \mathrm{M}_{\infty}\right)$ Versus $1 /\left(\mathrm{M}_{\mathrm{T}} / \mathrm{M}_{\infty}\right)$ FOR THE Gelatin $/ 15.8 \%$ FRUCTOSE MATRICES LOADED WITH GREEN DYE.

Figure 107. Plot OF $\mathrm{D}\left(\mathrm{M}_{\mathrm{T}} / \mathrm{M}_{\infty}\right)$ VERSUs $1 /\left(\mathrm{M}_{\mathrm{T}} / \mathrm{M}_{\infty}\right)$ FOR THE GELATIN $/ 0.037 \%$ FORMALDEHYDE MATRICES LOADED WITH GREEN DYE.

Figure 108. Plot OF D( $\left(\mathrm{M}_{\mathrm{T}} / \mathrm{M}_{\infty}\right)$ Versus $1 /\left(\mathrm{M}_{\mathrm{T}} / \mathrm{M}_{\infty}\right)$ FOR THE GELATIN MATRICES LOADED WITH RED DYE.

Figure 109. Plot OF $\mathrm{D}\left(\mathrm{M}_{\mathrm{T}} / \mathrm{M}_{\infty}\right)$ VERSUS $1 /\left(\mathrm{M}_{\mathrm{T}} / \mathrm{M}_{\infty}\right)$ FOR THE GELATIN $/ 30 \%$ SUCROSE MATRICES LOADED WITH RED DYE.

Figure 110. Plot Of $\mathrm{D}\left(\mathrm{M}_{\mathrm{T}} / \mathrm{M}_{\infty}\right)$ VERSUS $1 /\left(\mathrm{M}_{\mathrm{T}} / \mathrm{M}_{\infty}\right)$ FOR THE GELATIN $/ 15.8 \%$ FRUCTOSE MATRICES LOADED WITH RED DYE

Figure 111 . Plot OF $\mathrm{D}\left(\mathrm{M}_{\mathrm{T}} / \mathrm{M}_{\infty}\right)$ Versus $1 /\left(\mathrm{M}_{\mathrm{T}} / \mathrm{M}_{\infty}\right)$ FOR THE Gelatin $/ 0.037 \%$ FORMALDEHYDE MATRICES LOADED WITH RED DYE

FIGURE 112. LINEAR REGRESSION FOR THE HIGUCHI SQUARE ROOT EQUATION OVER THE FIRST PHASE (HOURS 4-20) FOR MATRICES LOADED WITH FLUORESCEIN 254

FIGURE 113. LINEAR REGRESSION FOR THE HIGUCHI SQUARE ROOT EQUATION OVER THE SECOND PHASE (HOURS 24-136) FOR MATRICES LOADED WITH FLUORESCEIN. 


\section{LIST OF APPENDICES}

\section{APPENDIX A}

APPENDIX 1 NMR SPECTRA OF NON-CROSS-LINKED GELATIN (A), GELATIN/15.8\%

FRUCTOSE (B), AND DIFFERENCE OF THE GELATIN AND GELATIN/15.8\% FRUCTOSE (C).

APPENDIX 2. NMR SPECTRA OF GELATIN/0.037\% FORMALDEHYDE MATRICES

APPENDIX B

APPENDIX 3. STANDARD CURVE FOR GREEN DYE.

APPENDIX 4. STANDARD CURVE FOR RED DYE.

APPENDIX C

APPENDIX 5. STANDARD CURVE FOR HIGH CONCENTRATIONS OF FLUORESCEIN.

APPENDIX 6. STANDARD CURVE FOR LOW CONCENTRATION OF FLUORESCEIN.

APPENDIX 7. SAMPLE CHROMATOGRAMS FOR A 30 NG STANDARD (A), A 750 NG STANDARD

(B), AND AN UNKNOWN SAMPLE (C) OF FLUORESCEIN.

276 


\section{Chapter I}

\section{Introduction and Literature Review}

\section{Introduction}

Traditional therapies, such as oral and intravenous routes, have been used to deliver many different drugs. However, these routes have limitations that make it difficult to safely and effectively deliver certain agents while maintaining patient convenience. Some limitations include insufficient plasma concentrations to be effective due to degradation by enzymes in the gastrointestinal tract and/or unpredictable, erratic absorption. The administration of the therapeutic agent by a medical professional is another limitation of traditional therapies. In order for an orally administered drug to reach the appropriate site of action, the drug must be absorbed in the gastrointestinal tract. For the drug component of a tablet to become fully available for absorption from the gastrointestinal tract, the tablet must first disintegrate and discharge the drug to the body fluids for dissolution [1]. Once dissolved in the gastrointestinal fluids, the drug must pass through a number of membrane barriers of the stomach and/or small and large intestine, gain entrance into the general circulation, and pass to the organ or tissue with which it exerts its desired effects. Once again, the drug must pass through a number of membrane barriers found in the heart, lung, or brain in order to advance into the tissue and enter into its individual cells. While the drug is in the gastrointestinal tract, the drug may be subjected to enzymes that can degrade the drug into either an active or inactive component, or, once in circulation, be changed by first-pass metabolism of the liver. 
The therapeutic agents also have limitations that can hinder their delivery into the body. Such limitations include physicochemical properties, undesirable side effects, and inconvenient dosage regimens. Physicochemical properties which can affect the dissolution of the drug include the surface area of the drug particle, drug solubility, the crystal or amorphous drug form, the salt form of the drug, the state of hydration, and molecular size. A structured and crystalline drug is less soluble than an amorphous drug. The salt form of the drug directly affects the solubility of the drug. For example, hydrochloride salts of weak bases and sodium and potassium salts of weak acids are more soluble than their parent compounds.

A localized delivery system could be designed to overcome these limitations. For example, solubility issues can be resolved by slowing release for highly soluble drugs. Drugs with high solubility are rapidly converted from the solid form to a molecular solution of the drug. A localized delivery system can control release by designing a matrix that is tightly cross-linked with the drug embedded within the matrix. On the other hand, low solubility drugs can be incorporated into a delivery system that provides release of drug with maximum exposure of the drug to the solvent. The placement of drug at a direct site may also offer the advantage of a favorable $\mathrm{pH}$ which can enhance the solubility of a poorly soluble drug. Drug release from these systems can be controlled by the properties of the matrix and is not totally dependent on the solubility of the drug. A localized delivery system can overcome unwanted side effects by reducing the amount of drug loaded into the delivery system by as much as 1,000 fold [5]. Patient compliance can also be greatly increased with a localized delivery system due to the ability to design 
a system that can release drug over a prolonged period. The ability to overcome these limitations has led to increased research in the area of localized or targeted drug delivery.

The goals for an ideal localized drug delivery system include predictable drug release throughout the desired time frame (likely sustained release), retention of delivery system intact during drug release followed by bioerosion, and little effect of the delivery system material on soft tissue at the site. Delivery systems intended for localized drug placement require specific design criteria in order to optimize treatment efficacy. The delivery system should be easy to administer and biodegrade so that the removal of the delivery system at a later time is not necessary. The delivery system should allow for delivery of various therapeutic agents to ensure the treatment of various disease processes that may be occurring at the site. The system should be equally, if not more, effective than conventional treatment methods.

In addition, varying manufacturing criteria need to be considered when designing a localized delivery system. The system should be easy to formulate, scale-up, and have manufacture reproducibility. The system should also be robust such that slight manufacturing deviations will not affect the delivery system quality and performance, and it should be cost effective. Potential applications for localized drug delivery include targeting mucosal tissues such as ophthalmic, nasal, gastrointestinal, urinary epithelia, mouth and skin [2-5]. 
A localized delivery system allows the drug to be targeted to a direct site while improving the efficacy of the drug, reducing the amount of drug needed, and lowering the incidence of side effects. The treatment of cancer is an area of interest for the utilization of a localized delivery system. The ability to deliver a lower concentration of a therapeutic agent directly to the tumor site while reducing the more toxic side effects (such as cardiotoxicity) offers a great advantage over conventional therapy. An implantable gelatin matrix cross-linked with glutaraldehyde was developed to target doxorubicin directly at the tumor site for the treatment of bone sarcoma [5]. The authors demonstrated that the biodegradable matrix had a substantially lower release rate of the doxorubicin due to cross-linking of the drug. Hanes, et al [6] developed gelatin and chondroitin sulfate microspheres that delivered interleukin- 2 for up to 21 days after being intratumorally injected into the brain and liver. The use of polymeric microspheres can eliminate the need to transfect each patient's cells with the cytokine gene, ultimately leading to a simplified therapeutic regimen.

Another area of interest for locally delivering chemotherapeutic agents is in the eradication of malignant tissues that cannot be completely removed after surgery, even in radical surgery. A localized delivery system that slowly releases drug directly to the tissue site where the malignant cells remain can be a highly effective post-surgery treatment. Okino, et al [7] developed a visible-light-driven photocurable tissue adhesive drug release system with a potential application for post-operative cancer chemotherapy. The authors focused on the fundamental aspects of styrene-derivatized gelatin and in vitro performance of the gel as a tissue-adhesive, drug releasable matrix. The use of a 
photo-curing or photopolymerization technique offers an advantage over conventional chemical cross-linking in that these methods have superior control over the gelation process [7].

Traditionally, periodontal disease has been treated with systemically administered antibiotics. However, in order for systemically administered drugs to reach the periodontal pocket these must pass through two barriers: the gut wall and the periodontal pocket mucosa. In order to overcome these disadvantages, local delivery devices, such as fibers, strips, films, injectable systems, microparticles, and gels, were developed to deliver drug directly to the periodontal pocket. Tewari [8] demonstrated the viability of a gelatin-based film, strengthened by formaldehyde cross-linking, for use in a sustained local delivery of clindamycin in the treatment of periodontal disease. Along with formaldehyde, a series of freeze-thaw curing steps were used in the manufacturing of films to give increased film rigidity. The added cross-linked structure of the gelatin matrix slowed its bioerosion and prolonged drug release from the film to 7-8 days [8]. Goodson, et al, [9] developed a hollow fiber of cellulose acetate releasing tetracycline as a first order process. The reservoir did not control the rate of release in which $95 \%$ of the tetracycline was released in the first 2 hours.

Hydrogels are polymers that have the ability to swell in water or aqueous solvent systems and retain a significant fraction of water within their macromolecular structure [10]. Kopeček, et al. [11] developed biodegradable azoaromatic cross-linked comonomer hydrogels that bind to colonic mucosal lectins and release 5-aminosalicylic acid in the 
colon by microbial enzyme degradation present in the colon. Stevens, et al [12] evaluated a three dimensional hydrogel that utilizes photopolymerized polyethylene glycol diacrylate physically entangled with gelatin to make an interpenetrating network (IPNs). The authors also developed a gelatin-based hydrogel entangled with gelatin cross-linked with glutaraldehyde. The hydrogels were loaded with dexamethasone and evaluated for in vivo biocompatability and in vitro/vivo drug release. Einerson, et al [13] developed PEG-dial (polyethylene glycol-dialdehyde) modified gelatin, EDTAD (ethylenediaminetetraacetic anhydride) modified gelatin, PEG-dial-and-EDTAD modified gelatin and evaluated the effect that environmental $\mathrm{pH}$, gelatin modification and cross-linking modality, in vitro swelling/degradation and drug release characteristics, in vivo degradation, inflammatory response and drug release activity. The authors were able to correlate the physicochemical properties of the hydrogels to the in vivo response.

To date, ophthalmic preparations such as solutions and suspensions are highly effective and commonly used delivery systems to treat glaucoma as well as acute and chronic infections of the eye. However, they are rapidly cleared from the eye surface, due to the movements of the eyelid and drainage of the lacrimal fluid, significantly reducing the contact time between the preparation instilled and the conjunctival and corneal epithelia [14, 15]. Hämäläinen, et al. [3] evaluated the use of Gelfoam ${ }^{\circledR}$ and monoisopropyl ester of poly(vinylmethyl ether/maleic anhydride) (PVM/MA) as polymers for ocular inserts. The authors found that drug release from the synthetic polymer (PVM/MA) was erosion controlled while the natural polymer (Gelfoam $\left.{ }^{\circledR}\right)$ demonstrated diffusion controlled release. Jaffe et al [16] demonstrated that a 
fluocinolone acetonide pellet coated with a polyvinyl alcohol crystalline structure and $\gamma$ ray irradiated to sterilize the device can be used to treat patients with uveitis. The authors observed favorable effects on inflammation, preservation or improvement of visual acuity, reversal of cystoid macular edema, and reduction/elimination of topical, periocular, or systemic anti-inflammatory agents.

The oral mucosa provides an alternative way to deliver drugs that are destroyed by the gastric juices or poorly absorbed from the gastrointestinal tract. McQuinn, et al. [17] developed a bioadhesive patch containing buprenorphine for the treatment of chronic or acute pain. The authors found that the patch demonstrated satisfactory tolerance and safety with mild to moderate adverse effects. Utilizing the same patch, Bénès, et al. [18] demonstrated that the transmucosal patch is an effective way to deliver drug while avoiding first pass metabolism.

\section{Literature Review}

\section{Natural Polymers - Gelatin}

Gelatin has been used in pharmaceutical delivery systems as the primary matrix for hard and soft gelatin capsules for immediate release, and as absorbable sponges or powders, plasma expanders and wound dressings. Natural polymers are ideal materials for the delivery of therapeutic agents at a localized site. Natural polymers, such as gelatin, provide a biodegradable sustained release delivery system that is relatively inexpensive and non-toxic. The interest in gelatin arises mainly from the fact that this natural polymer allows for the production of a biocompatible and biodegradable drug delivery system [19]. Gelatin has been shown to cause little to no allergic responses when placed 
in contact with soft tissues and softens upon wetting, factors which contribute to its biocompatibility.

Gelatin is obtained by a controlled hydrolysis of the fibrous insoluble protein, collagen, which is widely found in nature and is the major constituent of skin, bones, and connective tissue [20]. The collagen molecule consists of three polypeptide chains, alpha chains, that spontaneously coil together, zipper fashion, to form a right-handed helix [21, 22]. The strands are held together primarily by hydrogen bonds between adjacent $-\mathrm{CO}$ and -NH groups, but also by covalent bonds [23].

The conversion of collagen to gelatin occurs through one of two processes: an acid process or an alkaline process. The acid process is one in which the collagen is acidified to about $\mathrm{pH} 4$ and then heated stepwise from $50^{\circ} \mathrm{C}$ to boiling to denature and solubilize the collagen $[24,25]$. The collagen or gelatin solution needs to be defatted, filtered to high clarity, and concentrated by vacuum evaporation or membrane ultrafiltration treatment to a reasonably high concentration for gelation and then drying by passing dry air over the gel. The final process is one of grinding and blending. The alkaline process is one in which the collagen is submitted to a lengthy liming process prior to extraction [24]. After the alkaline processing, the collagen is washed free of alkali and treated with acid to the desired extraction $\mathrm{pH}$ (which has a marked effect on the gel strength to viscosity ratio of the final product). The collagen is then denatured and converted to gelatin by heating, as with the acid process. Because of the alkaline treatment, it is often necessary to demineralize the gelatin solution to remove excessive 
amounts of salts using ion-exchange or ultra-filtration. Thereafter the process is the same as for the acid process. The collagen to gelatin transformation generally produces a mixture of single alpha ( $\mathrm{MW}=80,000$ to 125,000 ), double alpha (also known as beta, $\mathrm{MW}=160,000$ to 250,000 ), and triple alpha (also known as gamma, $\mathrm{MW}=240,000$ to $375,000)$ stranded gelatin [25]. All the molecular species resulting from the processing of collagen to gelatin are considered to be gelatin provided that the molecular weight exceeds an arbitrary minimum of approximately 30,000 [26].

The protein backbone of gelatin consists of small percentages of each amino acid with the most frequent sequence being -gly-pro-R- (Figure 1) [27]. In general, gelatin consists of glycine $26.5 \%$, proline $16.2 \%$, hydroxyproline $13.5 \%$, glutamic acid $11.0 \%$, alanine $9.2 \%$, arginine $9.0 \%$, aspartic acid $6.2 \%$, lysine $5.0 \%$, serine $3.5 \%$, leucine $3.4 \%$, valine $2.6 \%$, phenylalanine $2.4 \%$, threonine $2.1 \%$, isoleucine $1.6 \%$, hydroxylysine $1.1 \%$, tyrosine, methionine, and histidine $0.8 \%$, and cysteine $0.2 \%$ [26]. The terminal groups of the gelatin strands have been extensively studied in the 1950's and 1960's. Serine, theronine, aspartic acid, and glutamic acid are predominate at the N-terminal in alkali processed gelatin and alanine in the acid processed gelatin [26]. At the C-terminal, glycine, glutamic acid, and aspartic acid are the predominate amino acids [26]. 


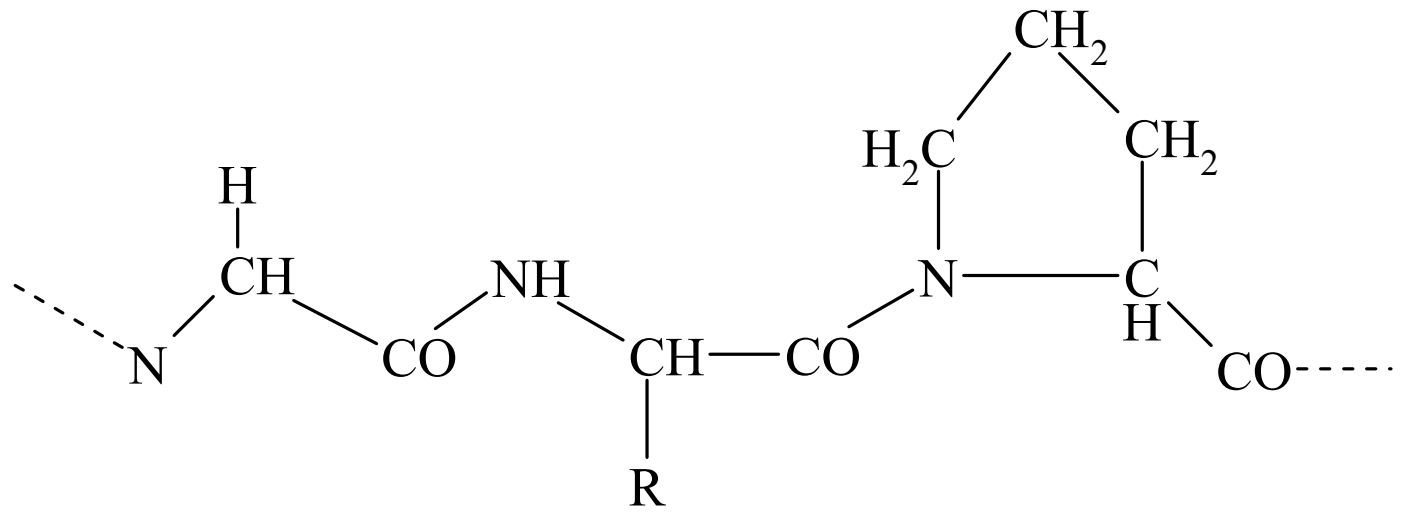

Figure 1. Protein backbone of gelatin where $R$ is any amino acid. 
The processing of collagen can produce two types of gelatin with different isoelectric points (pI). The alkaline process, through hydrolysis of the asparagine to aspartic acid and glutamine to glutamic acid, yields a gelatin (Type B) with a high density of carboxyl groups, which makes the gelatin negatively charged with an isoelectric point of 4.5-5.2 $[25,29]$. The acid process, which is less invasive, produces little change in the isoelectric nature of collagen. As a result, the isoelectric point of the gelatin (Type A) that is obtained will remain similar to that of collagen, between 7-9. Gelatin swells least at its isoelectric point and it swells more in solutions of $\mathrm{pH}$ above and below its $\mathrm{pI}$ [30].

\section{Gelatin Matrix - Formation and Behavior}

Gelatin has the ability to immobilize liquid and undergo gelation. Gelation is a disorder to order transition usually induced by cooling and is always reversible. Gelation begins with conformational changes which result in partial unfolding of gelatin within the solution, exposing the interior reactive region of the gelatin. As the gelatin is cooled, the unfolded proteins create a network throughout the system through intermolecular contacts or covalent bonds. The network is then stabilized by secondary forces rather than by covalent bonds [31]. The intermolecular attractions are negligible [31]. As the molecular network develops, additional gelatin molecules become attracted through electrostatic interactions. This ultimately leads to a more rigid structure and thickening of the gel. The maturation process involves two processes: an increasing order and stiffening of the network and general thickening of the whole network. As the gel continues to cool, additional parts of the gelatin chain become more ordered and stable due to the growth of existing links and formation of new links (both physical and chemical bonds) [31]. The

decrease in temperature causes the water molecules to be less mobile, the hydrogen bonds 
remain intact, ultimately rendering the chains immobile. The ability of the chains to continue to re-organize is lost. A slow cooling rate produces a strong, stable network.

Russo [32] morphologically classified gelatin as a fishnet gel (Figure 2) in which cross-links, whether reversible or covalent, provide 'strong points' of the structure (formation of crystallites) and are separated by flexible strands which provide elasticity. The strong points within the gelatin strands are due to intermolecular cross-linking (interstrand) that can occur between arginine-lysine or arginine-arginine within the same strand (Figure 3). Amino acid residues from two neighboring strands may also interact and form intramolecular (intrastrand) cross-linked strands that provide strength to the gelatin.

Two physical processes occur when a gelatin matrix is exposed to water. The first phase is the transition to a rubbery state as water penetrates the gelatin matrix and mobilizes the entangled strands [33]. The second phase is the continual swelling of the rubbery network as more water is absorbed. The rate of water absorption will be governed by $\mathrm{pH}$ of the environment, gelatin isoelectric point $(\mathrm{pI})$, moisture content, particle size, presence of dissolved salts, and the extent of cross-linking. The $\mathrm{pH}$ and the isoelectric point are important because when the $\mathrm{pH}$ of the environment and the isoelectric point are equal there is a net neutral charge on the gelatin. At this point, there is minimal absorption and swelling. Extensive swelling is seen when the $\mathrm{pH}$ deviates from the $\mathrm{pI}$ range. The extent of cross-linking is an essential characteristic of the delivery system due to its ability to reduce polymer chain mobility, increase glass transition 


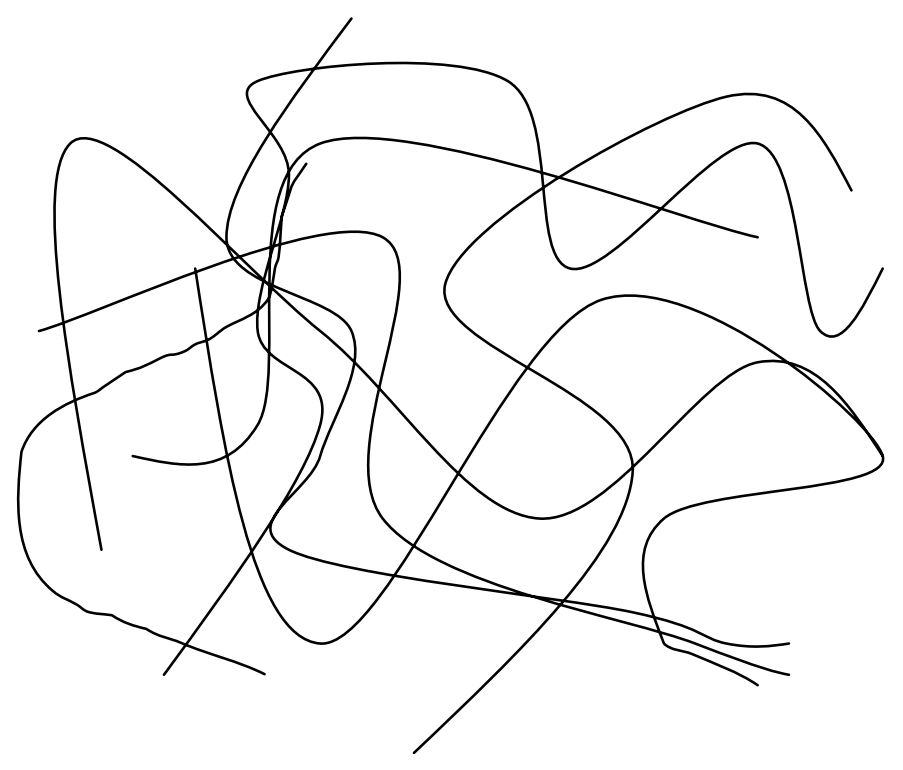

Figure 2. Fishnet structure of gelatin with strong points separated by flexible strands. 


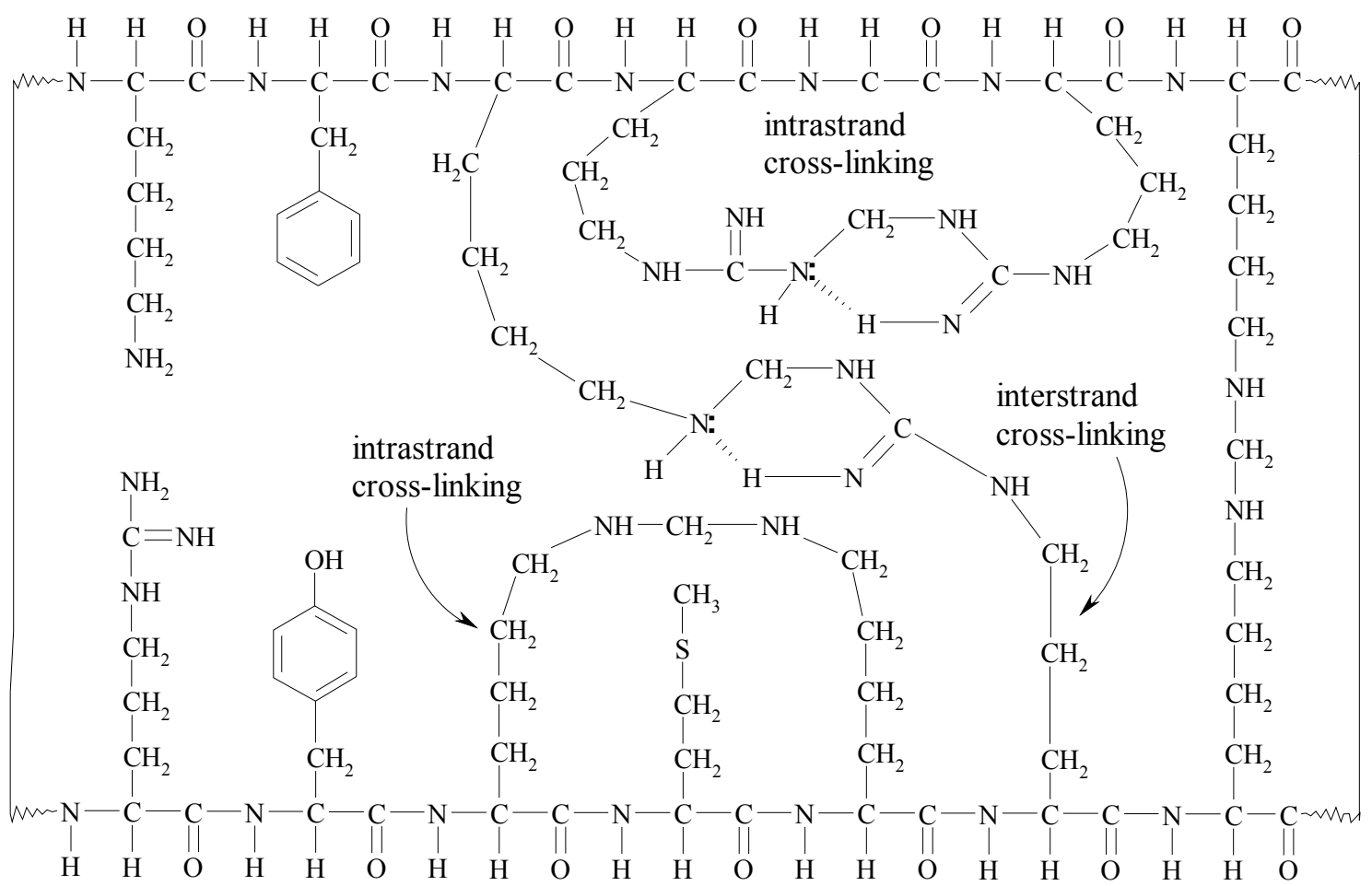

Figure 3. Inter- and intrastrand cross-linking within the gelatin. 
temperature and decrease molecular diffusivity [34].

The dissolution of a polymer into water involves two transport processes, solvent diffusion and chain disentanglement [35]. The dissolution of an uncross-linked, amorphous, glassy polymer occurs when a solvent diffuses into the polymer forming a gel-like layer. This layer is formed due to the increased mobility of the polymer strands and is contiguous to the solvent-polymer front. After an induction time (dissolution is not immediate upon polymer exposure to water), the polymer dissolves [35].

Semicrystalline polymers, such as cross-linked gelatin, undergo a slightly different dissolution process. Semicrystalline polymers contain both amorphous and crystalline sections spread throughout the polymer. An additional step, unfolding of the crystalline regions, must take place in order for the polymer to dissolve when exposed to a solvent. The penetration of solvent into the amorphous sections is fast and extensive because of their lower density, producing high initial swelling rates and leading to high equilibrium uptakes [36]. Crystalline regions are more tightly ordered with a higher density resulting in a limited and slower penetration of the solvent. The equilibrium swelling of the crystalline sections is considerably less extensive than that of the amorphous regions because the regular arrangement of the polymer chains in the crystallites results in greater lateral interchain attraction and greater elastic forces resisting expansion and swelling [36]. The final step in the dissolution process is chain disentanglement in which the polymer structure falls apart and dissolves. 
The dissolution of polymer and non-polymer materials differs in two ways. Polymers require an induction time before starting to dissolve, while non-polymeric materials can dissolve instantaneously [35]. The external mass transfer resistance through a liquid layer adjacent to the solid-liquid interface (diffusion layer and its thickness) generally controls non-polymeric material dissolution. Polymer dissolution can be controlled either by the disentanglement of the polymer chains or by diffusion of the chains through a boundary layer adjacent to the solvent-polymer interface [35]. These processes contribute to the induction time prior to the polymer dissolution.

The main limitation to gelatin for the preparation of sustained release systems arises from its rapid dissolution and drug release in aqueous environments at physiological temperatures [19]. The rate and extent to which gelatin swells dictates, in part, the amount of drug released and the rate at which that occurs. To surmount this limitation, changes must be made in gelatin's physicochemical properties. Non-soluble networks can be formed using chemical cross-linking agents [5, 20, 37-41] or thermal hardening treatment $[42,43]$. While traditional chemical cross-linking agents (formaldehyde and glutaraldehyde) show promise, concerns exist about their unwanted reactions with drugs [42] and the potential for irritable effects at the site of action [39, 44-45]. Due to these concerns, the need arises for a localized sustained release delivery system containing a non-toxic, inert chemical cross-linking agent.

Cortesi, et al. [19] reported that the use of native and oxidized sugars were able to reduce the dissolution of gelatin and could be a method to cross-link gelatin, thereby 
reducing the risk of toxic side effects due to the use of synthetic cross-linkers. Native sugars such as sucrose and fructose can provide an alternative method for cross-linking. Sugars are non-toxic, readily available, commonly used in the human diet, and biocompatible. Sucrose is a dissacharide which can undergo acid hydrolysis and yield one D-glucose and one D-fructose (Figure 4a). Sucrose has a melting temperature of $185-187^{\circ} \mathrm{C}$. Sucrose is not a reducing sugar. Consequently, neither of the rings are able to open. Sucrose does not exhibit mutarotation, implying that sucrose has no hemiacetal linkages and that glucose and fructose must be glycosides. This can only happen if the two sugars are joined by a glycosidic link between 1-C of glucose and 2-C of fructose. Sucrose is hygroscopic and can absorb up to $10 \%$ moisture which is given up on heating to $90^{\circ} \mathrm{C}$.

Fructose is a highly soluble monosaccharide with a melting temperature of 122$125^{\circ} \mathrm{C}$ (Figure $4 \mathrm{~b}$ ). Under basic conditions, the fructose molecule can have the location of the carbonyl bond switched to convert the fructose into a glucose molecule. Monosaccharides, such as fructose, that are in the ring form are not reducing sugars because they do not have the aldehydro group that can be oxidized. However, because they are in equilibrium with the open form, any monosaccharide in the ring form will, within a fraction of a second, be in the open form and, thus, be able to react with the oxidizing agent and reduce it.

The mechanism for gelatin/sugar cross-linking may occur by one of two reactions as reported by Digenis, et al. [46]. One possible mechanism involves aldose sugars 


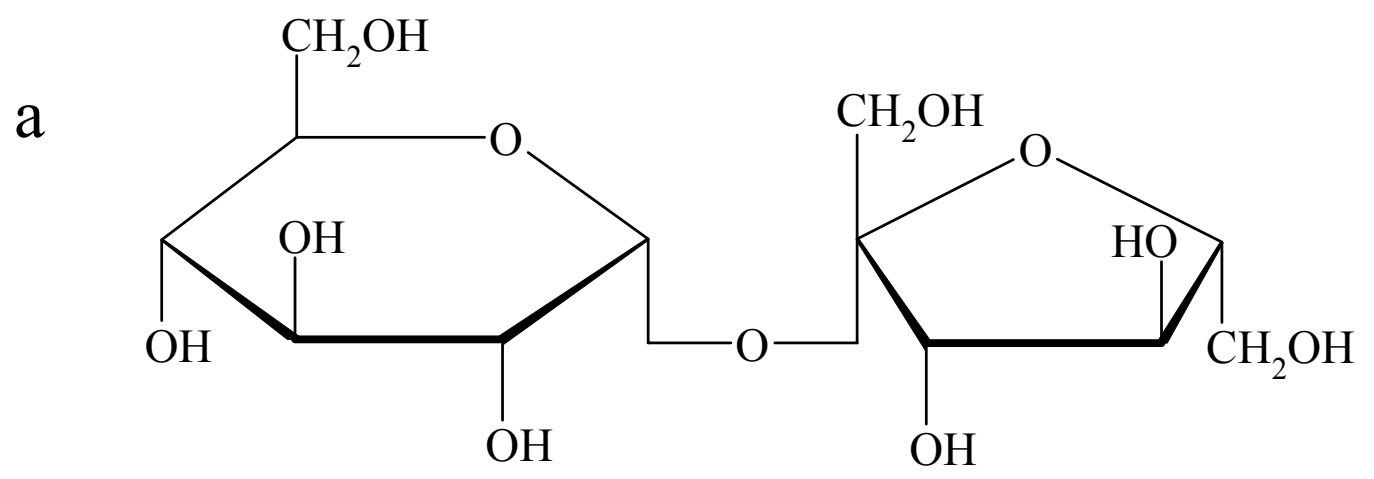

b

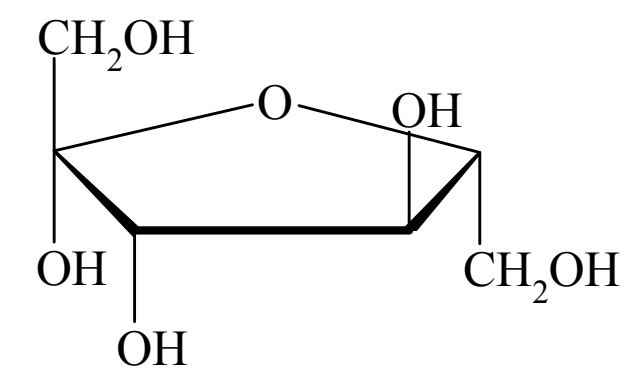

Figure 4. Structure of sucrose (a) and fructose (b). 
(Figure 5). The aldehyde group of the sugar can react with the free $\varepsilon$-amino group on the side chains of the gelatin molecule, undergoing an Amadori rearrangement [47], resulting in the formation of an aminoglycoside [46]. The aminoglycoside can then further react with another amino group forming its cross-linked structure. The other possible reaction mechanism, presented in Chapter $\mathrm{V}$, also describes the mechanism which formaldehyde cross-links with gelatin. The extent of cross-linking is difficult to control due to gelatin's ability to interact both chemically and physically with the same strand or adjacent strands as well as with the cross-linking agents. One way to control this would be to react equal molar concentrations of gelatin (particularly lysine groups) with equal molar concentrations of cross-linking agent.

The determination of the mechanisms that control drug release and the mathematical modeling of gelatin-based delivery systems is difficult because of the complexity of the system. Drug release from a biodegradable, polymeric controlled release delivery device can be either (a) diffusion controlled, (b) swelling controlled, or (c) erosion controlled or a combination of these mechanisms. Erodible delivery systems are complex and usually incorporate more than one of these processes in the kinetics of the drug's release profile. The chemical reactions, such as polymer degradation, may also play a role in the release characteristics of the gelatin matrix [48].

A homogeneous matrix is defined as a matrix in which the drug is intimately mixed within the polymer and release occurs by diffusion from the matrix. Diffusion is a concentration dependent process in which individual molecules are transferred from the 


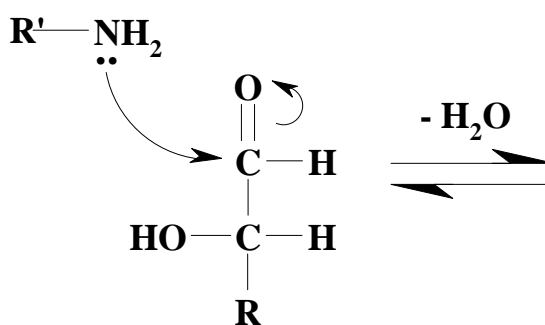

aldose sugar

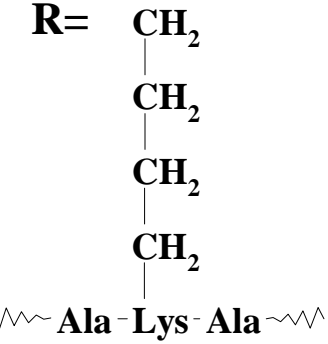

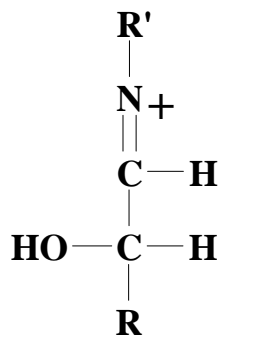

imine

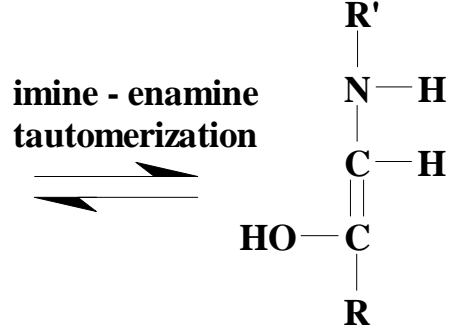

enamine (enol)

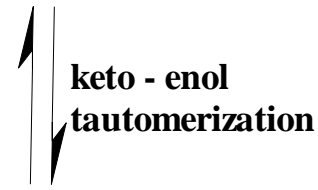<smiles>[R][R]C(C)(C)[Ge]</smiles>

Figure 5. Schematic representation of the cross-linking reaction mechanism between the sugars and gelatin. 
matrix into the solvent by random molecular motion. Drug release from a homogeneous matrix assumes that dissolution is initiated at the solid-liquid front and gradually the front moves through subsequent layers to the inner core [49].

In swelling controlled release, the mechanism of release depends on the polymer existing in a glassy state with drug entrapped within the matrix. Swelling controlled release is controlled by the velocity of the inner moving water front [50]. Both hydrophobic and hydrophilic matrices generally exhibit this swelling phenomenon during drug release in aqueous environments. After time, an equilibrium state in which the elastic and osmotic (swelling) forces are equal is seen in matrices that have polymer strands that are chemically or physically interacting.

Erosion controlled release is based on the loss of the material from the polymer bulk. Currently, there are two recognized types of erosion behavior: (a) bulk or homogeneous erosion and (b) surface or heterogeneous erosion. During surface erosion, degradation occurs predominantly on the outside of the matrix and is much faster than the water imbibition into the polymer bulk. In bulk erosion, degradation is slow because the entire device is rapidly wetted and chain cleavage occurs throughout the matrix. Different physicochemical processes can be involved in drug release depending on the design of the device (composition and geometry) [48].

Finally, depending on the composition of the delivery system (polymer type, drug loading, additives) and geometry (size and shape), numerous transport and chemical 
reaction phenomena can affect the resulting drug release kinetics [51]. These include, but are not limited to, water intrusion/polymer swelling, creation of polymer pores, polymer degradation, drug dissolution, diffusion of drug and/or polymer degradation products inside the polymer matrix and/or pores, polymer and drug net charge, osmotic effects, convection, and adsorption/desorption processes. A comprehensive mechanistic analysis of matrix performance would need to identify the relevant phenomena and include these in the treatment.

\section{Gelatin Matrix - Delivery Applications}

A gelatin matrix cross-linked with inert, native sugars has several possible applications as a localized drug delivery system. The treatment of periodontal disease, decubitus ulcers, and ophthalmic diseases are three areas which could benefit from a sustained release, biodegradable, biocompatible localized delivery system.

Biodegradable delivery systems offer the advantage of being cost effective because they do not require the removal of the delivery device.

The first disease state that the gelatin/sugar matrices could be utilized is in periodontal disease. Periodontal disease is a general term used to describe specific diseases that affect the gingiva, the supporting connective tissue, and alveolar bone which anchors the teeth in the jaws [52]. Periodontal disease begins with gingivitis, which only involves the gingiva and can progress to periodontitis. This is a common infection among the adult population. If left untreated, it can continue to progress to advanced periodontitis where the teeth become totally detached from the supporting bone and fall out. 
Periodontitis occurs when pockets form due to the inner layer of the gum and bone pulling away from the teeth [53]. The small spaces between the gum and teeth collect debris and can become infected. The body's immune system fights the bacteria as the plaque spreads and grows below the gum line. As the plaque continues to spread, the bacteria are producing toxins and enzymes that break down the bone and connective tissue that hold the teeth in place. As the disease progresses, the pockets deepen and more gum tissue and bone is destroyed. When this happens, the teeth are no longer anchored in place, become looser, and tooth loss occurs [53].

Current treatment of periodontitis includes non-surgical as well as surgical therapy. Non-surgical therapy includes a dental cleaning by a dental professional or scaling and root planing. Scaling and root planing is a procedure, requiring local anesthesia, in which the plaque and tartar that form above and below the gum line are scraped away and the rough spots on the tooth root are made smooth. Smoothing the rough spots removes bacteria and provides a clean surface for the gums to reattach to the teeth.

Surgical procedures include flap reduction and pocket reduction surgery where the gum is pulled away from tooth, the plaque and bacteria are removed, and then the gum is replaced. Bone grafts can be done and serves as a platform for the re-growth of bone which restores stability to the teeth. Soft tissue grafts involve the removal of skin from the mouth, usually from the roof of the mouth, that is then grafted around the affected area, reinforcing thin gums or filling in places where gums have receded. When 
the bone supporting the teeth has been destroyed, guided tissue regeneration (GTR) helps to stimulate bone and tissue growth. It is usually done in combination with flap reduction. In GTR, a small mesh-like fabric is inserted between the bone and gum tissue to keep the gum tissue from growing into the area where the bone should be, allowing the bone and connective tissue to re-grow to better support the teeth. The last treatment option is bone surgery in which the shallow craters, due to moderate bone loss, are smoothed and reshaped to decrease the craters. This makes it more difficult for the bacteria to collect and grow.

Current medicinal therapy for the treatment of periodontal disease is systemic antibiotics, topical antibiotics, or topical antiseptics. Antibiotics have been used in combination with surgery and other therapies, or alone to reduce/temporarily eliminate the bacteria associated with periodontal disease or suppress the destruction of the tooth's attachment to the bone. Systemically administered antimicrobials have been advocated for the treatment of severe forms of periodontitis, however, concerns existed about systemic therapy for chronic infections [54]. The benefits of the antimicrobial effects do not outweigh the side effects including hypersensitivity, gastrointestinal intolerance, and the development of bacterial resistance [55].

Applying the drug directly to the tissue or bone site can reduce the side effects as well as significantly increase the local tissue concentration. This type of system may have applications where systemic drugs are currently used such as in localized juvenile periodontitis, refractory periodontitis, and periodontitis with secondary systemic 
involvement [54]. However, some studies have reported poor results due to the fact that the active product could not achieve an adequate concentration at the site of action (periodontal pocket) and/or due to the inability of the active product to be retained locally in this pocket for a sufficient period of time [56].

The antibiotics of choice used to treat periodontal disease include chlorhexidine, doxycycline, tetracycline, clindamycin, minocycline and metronidazole. Traditional methods of delivery have either been the oral route or localized rinses. Recently, several commercially available products to treat periodontal disease have been marketed, but these either need to be removed at a later time or do not exhibit sustained release. Larsen [57] evaluated the in vitro release of doxycycline from biodegradable materials and acrylic strips. Goodson, et al [58] evaluated the performance of different polymer (polyethylene, polycaprolactone, polypropylene, polyurethane, cellulose acetate propionate, and ethylene vinyl acetate) fibers loaded with tetracycline. The authors found that the ethylene vinyl acetate (EVA) released drug over a 9 day period in vitro. This product has been marketed as Actisite ${ }^{\circledR}$ (tetracycline hydrochloride) (Proctor \& Gamble, Cincinnati, $\mathrm{OH}$ ) but requires lengthy treatment time in its application and must be removed at a later time. Chlorhexidine is an antimicrobial used to control plaque and gingivitis in the mouth or in periodontal pockets. It is commercially available as a mouth rinse or as a gelatin-filled chip (Periochip®, Astra Pharmaceuticals, Wayne PA) that is placed in the pockets after root planing and releases the medication slowly over 7 days. To date, Atridox ${ }^{\circledR}$ (doxycycline hyclate) (Atrix Labs, Fort Collins, CO) and Periochip ${ }^{\circledR}$ 
are the only controlled release biodegradable delivery systems available for treatment of periodontal disease.

Research continues to be done in this area in order to develop a delivery system that can reach deep into the periodontal pocket, possess high concentration of drug over an extended period of time, and biodegrade. For these reasons, the gelatin/sugar matrix can be beneficial in the treatment of periodontal disease, due to its ability to conform to the site upon warming, providing another treatment option. The gelatin/sugar matrices can be placed deep in the periodontal pocket and begin to conform to the area. This is important in periodontal disease because the pocket may not be a nice, neat, square or circular area. They also have the advantage of being collagen-based and may help in the regeneration process of the fibrous tissue that is lost in the advanced stages of the disease.

The second disease state in which the gelatin/sugar matrices may be beneficial is in the treatment of decubitus ulcers. Decubitus ulcers, also known as pressure sores, are defined as an ulceration of the skin and underlying tissues caused by prolonged pressure over the affected area [59]. Pressure ulcers occur when unrelieved pressure on the skin squeezes tiny blood vessels which supply the skin with nutrients and oxygen. When the skin is starved of nutrients and oxygen for too long, the tissue dies and a pressure ulcer forms. The skin is not a uniform organ. Its appearance and thickness vary according to the site, making particular areas of the skin more susceptible to pressure sores. The ulcerations usually occur over bony prominences such as heels, spine, coccyx area of the buttocks, lower back, shoulder blades, elbows, and even the back of the head [60]. 
Pressure sores can range in severity from mild (redness of the skin) to severe (deep craters down to the muscle and bone). Pressure ulcers are serious problems that can lead to pain, a longer stay in the hospital or nursing home, and slower recovery from health problems.

The treatment of pressure sores includes relieving the pressure by rotating the pressure point, as well as good nutrition (poor nutrition can lead to the breakdown of skin). It is important to keep the sore clean. A pressure sore needs to be cleaned daily with saline solution [61]. Although the sore must be kept moist, unaffected tissue around it should be kept clean and dry (but moisturized) to prevent further damage. The removal of dead tissue and application of medicated ointments or creams helps reduce the buildup of bacteria and lowers the risk of infection. If infection develops, it is important to identify and treat the infection promptly with antibiotics. For severe pressure sores, surgery may be required [61]. The gelatin/sugar matrices have the potential to deliver anti-microbial agents directly to the pressure sore when infection begins, preventing further damage and promoting healing at the site. The size of the gelatin/sugar matrices can also be controlled such that a variety of sizes could be available for the treatment of the sores. The gelatin/sugar matrices also have the ability to absorb some of the exudate that may be produced through the course of the disease.

The third disease state in which the gelatin/sugar matrices could be utilized as a therapeutic device is in the treatment of ophthalmic disorders. There are several disorders, such as uveitis and scleritis, that could benefit from a localized delivery 
system. Studies have shown that an enhanced absorption into the aqueous humor is seen when ocular inserts are used compared to traditional eye drops [62, 63]. Friedrich, et al [64] evaluated the affect that ocular inserts had on other ocular tissues such as the irisciliary body and the conjunctiva. The authors found that the highest levels of timolol were detected in the cornea, followed by the conjunctiva, iris-ciliary body, aqueous humour and plasma. The amount of drug entering the iris-ciliary body and aqueous humour was higher when ocular inserts were used in place of eye drops. The authors conclude that this is likely due to an increase in the amount of drug which enters the interior of the eye through non-corneal penetration (conjunctiva and sclera).

Uveitis is the inflammation of one or more components of the uveal tract (iris, ciliary body, choroid) [65]. The iris is the colored part of the eye. The ciliary body is found behind the iris and is responsible for the fluid inside the eye. The choroid is the vascular lining tissue underneath the retina. The inflammation may be the result of diverse stimuli: traumatic, immunologic, or infectious. It may be classified as acute, recurrent, or chronic uveitis. Chronic uveitis is more common than acute or recurrent uveitis. The effect of inflammation on the ocular tissue depends to a certain degree on the pathophysiologic mechanisms responsible for the disease and the layer of the eye involved [65]. The inflammation process is divided into two categories, granulomatous and nongranulomatous, that refer to the appearance of precipitates within the eye. Granulomatous precipitates are large and yellowish white, while the nongranulomatous precipitates are small, fine, and whiter in color [66]. If uveitis is left untreated or 
undertreated, it can cause repeated episodes of inflammation within the eye and can lead to scarring and blindness.

Therapy for uveitis is tailored according to the area in which the inflammation occurs. Current therapy for severe uveitis includes systemic corticosteroids (prednisolone, dexamethasone, fluorometholone) which may be in combination with cycclosporine A or azathioprine. Treatment may include steroid eye drops, injections, or oral treatment as well as mydriatic drops (phenylephrine $\mathrm{HCl}$, atropine, homatropine, tropicamide) to dilate the pupil and non-steroidal drops (diclofenac, indomethacin, flurbiprofen, ketorolac) to reduce the pain. Periocular injections have the ability to rapidly deliver high concentrations of drug in an effective manner to treat severe inflammatory conditions. However, it has several disadvantages. These injections require the use of anesthesia, increase the risk of penetration into the globe by the needle, may cause orbital problems with chronic injections or cause severe intractable glaucoma and reactions to the vehicle may occur [66].

Kunou, et al [67] developed a PLGA (poly(D,L-lactide-co-glycolide) biodegradable scleral implant loaded with betamethasone phosphate for the treatment of chronic uveitis. DeCampos, et al [68] evaluated the performance of chitosan nanoparticles containing cyclosporine A for the delivery of drugs to the ocular mucosa. The nanoparticles were placed in the cul-de-sac in both eyes of rabbits. The authors found that the concentrations in the cornea and conjunctiva were statistically greater in rabbits treated with the nanoparticles than the rabbits treated with control eye drops. Lee, 
et al [69] developed a Gelfoam ${ }^{\circledR}$ based ocular disc that was instilled into the lower conjunctival sac in the rabbit's eye. The authors demonstrated that the administration of the disc produces a larger and longer lasting effect due to the ability of the disc to remain intact for a longer period of time than the traditional eye drops.

The second type of ophthalmic disorder to benefit from a local delivery system is scleritis. Scleritis is the inflammation located in the sclera of the eye which is the tough, white outer layer (coat) of the eyeball made up of connective tissue fibers. The inflammation may be the result of various stimuli, the most famous being rheumatoid arthritis. Many of the connective tissue diseases or collagen vascular diseases may have scleritis as part of their disease spectrum. As with uveitis, current therapy includes systemic non-steroidal anti-inflammatory agents for pain relief, immunosuppressive therapy, glucocorticoids (usually a second line agent due to side effects), and immunosuppressive agents (severe and resistant forms). The same therapeutic problems and limitations encountered with uveitis occur in scleritis. Current research is being conducted to improve ocular residence time of therapeutic agents in order to treat the inflammation.

Other ophthalmic diseases such as the treatment of cytomegalovirus retinitis may benefit from a localized delivery. Human cytomegalovirus (HCMV) retinitis is the most common ocular infection in patients with AIDS [70]. Sakurai, et al [71] evaluated the use of a biodegradable scleral plug containing ganciclovir. The authors found that the scleral plugs significantly reduced the inflammatory changes that occurred in HCMV 
retinitis. In addition, the authors reported that the minimal sclerotomy conducted to implant the plug did not cause any lasting problems and were replaced with fibrous tissue after the plug eroded away.

The gelatin/sugar matrices have applications in the ophthalmic disease states mentioned above because they have the ability to become soft and conform to the area of the eye in which they are applied. This makes the matrices comfortable in the eye and secure from spillage, dilution or drainage into the nasal cavity as is seen with eye drops, maintaining contact with the surface. The matrices can also be manufactured aseptically. A final advantage of the gelatin-based matrices is the ability to be developed as a delivery system that can utilize geometry differences as a function of delivery and delivery site needs. 


\section{References}

1. Ansel HC, Popovich NG, Allen LV Jr. In: Pharmaceutical Dosage Forms and Drug Delivery Systems. Philadelphia:Williams \& Wilkens, 1995. p. 190.

2. Kunou N, Ogura Y, Honda Y, Hyon S-H, Ikada Y. Biodegradable scleral implants for controlled ocular delivery of betamethasone phospate. J Biomed Mater Res 2000;51:635-641.

3. Hämäläinen KM, Määttä E, Piirainen H, Sarkola M, Väisänen A, Ranta V-P, Urtti A. Roles of acid/base nature and molecular weight in drug release from matrices of gelfoam and monoisopropyl ester of poly(vinyl methyl ether-maleic anhydride). $J$ Control Release 1998;56:273-283.

4. Schwach-Abdellaoui K, Vivien-Castioni N, Gurny R. Local delivery of antimicrobial agents for the treatment of periodontal diseases. Eur J Pharm Biopharm 2000;50:8399.

5. Fan H, Dash AK. Effect of cross-linking on the in vitro release kinetics of doxorubicin from gelatin implants. Int J Pharm 2001;213:103-16.

6. Hanes J, Sills A, Zhao Z, Suh KW, Tyler B, DiMeco F, Brat DJ, Choti MA, Leong $\mathrm{KW}$, Pardoll DM, Brem H. Controlled local delivery of interleukin-2 by biodegradable polymers protects animals from experimental brain tumors and liver tumors. Pharm Res. 2001;18(7):899-906.

7. Okino H, Nakayama $\mathrm{Y}$, Tanaka M, Matsuda. In situ hydrogelation of photocurable gelatin and drug release. J Biomed Mater Res. 2002;59:233-245.

8. Tewari R, Crout R, Meyer-Stout PJ. Evaluation of a gelatin based controlled release drug delivery system for the treatment of periodontal disease, M.S. Thesis, WVU School of Pharmacy, 1997.

9. Goodson JM, Haffajee A, Socransky SS. Periodontal therapy by local delivery of tetracycline containing fibers for controlled delivery to periodontal pockets. J Clin Periodontol. 1979;6:83-92.

10. Hui X. Pingtian D, Gang W, Guidheng Z, Junmin Z. Equibrilium swelling and solute diffusion characteristics of Poly (Methacrylic Acid co-Poloxamer) hydrogels. J Chin Pharm Sci 2001;10:203-207.

11. Kopeček J, Kopečková P, Brøndsted H, Rathi R, Řihová B, Yeh P-Y, Ikesue K. Polymers for colon-specific drug delivery. J Control Release 1992;19:121-130. 
12. Stevens KR, Einerson NJ, Burmania JA, Kao WJ. In vivo biocompatability of gelatinbased hydrogels and interpenetrating networks. J Biomater Sci Polymer Edn. 2002;13(12):1353-1366.

13. Einerson NJ, Stevens KR, Kso WJ. Synthesis and physicochemical analysis of gelatin-based hydrogels for drug carrier matrices. Biomaterials. 2002;24:509-523.

14. Chrai SS, Patton TF, Mehta A, Robinson JR. Lacrimal and instilled fluid dynamics in rabbit eyes. J Pharm Sci 1973;62:1112.

15. Merkli A, Tabatabay C, Gurny R. Use of insoluble biodegradable polymers in ophthalmic systems for the sustained release of drugs. Eur J Pharm Biopharm 1995;41:271-283.

16. Jaffe GJ, Ben-nun J, Guo H, Dunn JP, Ashton P. Fluocinolone acetonide sustained drug delivery device to treate severe uveitis. Ophthalmology. 2000;107:2024-2033.

17. McQuinn R, Kvam D, Maser M, Miller A, Oliver S. Sustained oral mucosal delivery in human volunteers of buprenorphine from a thin non-eroding mucoadhesive polymeric disk. J Control Release 1995;34:243-250.

18. Bénès L, Claustrat B, Horrière F, Geoffriau M, Konsil J, Parrott KA, DeGrande G, McQuinn RL, Ayres JW. Transmucosal, oral controlled-release, and transdermal drug administration in human subjects: A crossover study with melatonin. J Pharm Sci 1997;86:1115-1119.

19. Cortesi R, Nastruzzi C, Davis SS. Sugar cross-linked gelatin for controlled release: microspheres and disks. Biomaterials 1998;19:1641-9.

20. Choi YS, Hong SR, Lee YM, Song KW, Park MH, Nam YS. Study on gelatincontaining artificial skin: 1. Preparation and characteristics of novel gelatin-alginate sponge. Biomaterials 1999;20:409-17.

21. Lee CH, Singla A, Lee Y. Biomedical applications of collagen. IJP 2001;221:1-22.

22. Prockop DJ. What hold us together? Why do some of us fall apart? What can we do about it? Matrix Biol 1998;16(9):519-528.

23. Harkness RD. Biological functions of collagen. Biol Rev 1961;36:399-463.

24. Johns P, Courts A. Relationship between collagen and gelatin. In: Ward AG, Courts A, editors. The Science and Technology of Gelatin. London: Academic Press, 1977. p 137-177.

25. Veis A. In: The Macromolecular Chemistry of Gelatin. New York:Academic Press, 1964. 
26. Eastoe JE, Leach AA. Chemical constitution of gelatin. In: Ward AG, Courts A, editors. The Science and Technology of Gelatin. London: Academic Press, 1977. p 73-107.

27. Clark RC, Courts A. The chemical reactivity of gelatin. In: Ward AG, Courts A, editors. The Science and Technology of Gelatin. London: Academic Press, 1977. p. 209-47.

28. Merck Index $12^{\text {th }}$ ed; Budavari S, Ed; Merck:Rahway NJ, 1996; p 742-743.

29. Tabata Y, Ikada Y, Protein release from gelatin matrices. Adv Drug Del Rev 1998;31:287-301.

30. Welz MM, Ofner CM III. Examination of self-crosslinked gelatin as a hydrogel for controlled release. J Pharm Sci 1992;81:85-90.

31. Stainsby G. The gelatin gel and the sol-gel transformation. In: Ward AG, Courts A, editors. The Science and Technology of Gelatin. London: Academic Press, 1977 p. 179-207.

32. Russo PS. A perspective on reversible gels and related systems. ACS Symposium Series 1987;350:1-21.

33. Leucuta SE, Ponchel G, Duchêne D. Dynamic swelling behaviour of gelatin/poly (acrylic acid) bioadhesive microspheres loaded with oxprenolol. J Microencap 1997;14(4):501-510.

34. Deasy PB. In: Microencapsulation and related drug processes. New York:Marcel Dekker, 1984. p. 84-85.

35. Narasimhan B. Mathematical models describing polymer dissolution: components for drug delivery. Adv Drug Del Rev 2001;48:195-210.

36. Ofner III CM, Schott H. Swelling studies of gelatin I: gelatin without additives. $J$ Pharm Sci 1986;75(8):790-796.

37. Vandelli MA, Rivasi R, GuerraP, Forni F, Arletti R. Gelatin microspheres crosslinked with D,L-glyceraldehyde as a potential drug delivery system: preparation, characterisation, in vitro and in vivo studies. Int J Pharm 2001;215:175-184.

38. Dalev P, Vassileva E, Mark JE, Fakirov S. Enzymatic degradation of formaldehyde crosslinked gelatin. Biotechnology Techniques 1998;12:889-892.

39. Speer DP, Chvapil M, Eskelson CD, Ulreich J. Biological effects of residual glutaraldehyde in glutaraldehyde-tanned collagen biomaterials. J Biomed Mater Res 1980;14:753-764. 
40. Raymond G, Degennaro M, Mikeal R. Preparation of gelatin:phenytoin sodium microspheres: an in vitro and in vivo evaluation. Drug Dev Ind Pharm 1990;16:10251051.

41. Leo E, Vandelli MA, Cameroni R, Forni F. Doxorubicin-loaded gelatin nanoparticles stabilized by glutaraldehyde: Involvement of the drug in the cross-linking process. Int J Pharm 1997;155:75-82.

42. Esposito E, Cortesi R, Nastruzzi C. Gelatin microspheres: influence of preparation parameters and thermal treatment on chemico-physical and biopharmaceutical properties. Biomaterials 1996; 17:2009-2020.

43. Yannas IV, Tobolsky AV. Cross-linking of gelatin by dehydration. Nature 1967;215:509-510.

44. Sung HW, Huang D-M, Chang W-H, Huang R-N, Hsu J-C. Evaluation of gelatin hydrogel crosslinked with various crosslinking agents a bioadhesives: In vitro study. $J$ Biomed Mater Res 1999;46:520-530.

45. Ulubayram K, Aksu E, Gurhan EID, Serbetci K, Hasirci N. Cytotoxicity evaluation of gelatin sponges prepared with different cross-linking agents. J Biomater Sci Polymer Edn 2002;13(11):1203-1219.

46. Digenis GA, Gold TB, Shah VP. Cross-linking of gelatin capsules and its relevance to their in vitro-in vivo performance. J Pharm Sci 1994;83:915-21.

47. Hodge JE. The Amadori Rearrangement. Adv Carbohydrate Chem 1955;10:169-205.

48. Faisant N, Siepmann J, Benoit JP. PLGA-based microparticles: elucidation of mechanisms and a new, simple mathematical model quantifying drug release. Eur $J$ Pharm Sci 2002;15:355-366.

49. Chowdhury DK, Mitra AK. Kinetics of in vitro release of a model nucleoside deoxyridine from crosslinked insoluble collagen and collagen-gelatin microspheres. Int J Pharm 1999;193:113-122.

50. Klech CM, Li X. Consideration of drug load on the swelling kinetics of glassy gelatin matrices. J Pharm Sci 1990;79(11):999-1004.

51. Siepmann J, Göpferich A. Mathematical modeling of bioerodible, polymeric drug delivery systems. Adv Drug Del Rev 2001;48:229-247.

52. Williams RC. Periodontal disease N Engl J Med. 1990;322(6):373-382.

53. Watts TLP. In: Periodontics in Practice. London:Martin Dunitz, 2000. 
54. Schwach-Abdellaoui K, Vivien-Castioni N, Gurny R. Local delivery of antimicrobial agents for the treatment of periodontal diseases. Eur J Pharm Biopharm. 2000;50:8399.

55. Bollen CM, Quirynen M. Microbiological response to mechanical treatment in combination with adjunctive therapy. J Periodontol. 1996;67:1143-1158.

56. Vandekerckhove BNA, Quirynen M, VanSteenberghe D. The use of tetracyclinecontaining controlled release fibers in the treatment of refractory periodontitis. $J$ Periodontol. 1997;68:353-361.

57. Larsen T. In vitro release of doxycycline from bioabsorbable materials and acrylic strips. J Periodontol. 1990;61:30-34.

58. Goodson JM, Holborow D, Dunn RL, Hogan P, Dunham S. Monolithic tetracycline containing fibers for controlling delivery to periodontal pockets. $J$ Periodontol. $1983 ; 54: 575-579$.

59. Thomsa DR. The promise of topical growth factors in healing pressure ulcers. Ann Intern Med 2003;139(8):694-695.

60. Thomas DR. Issues and dilemmas in the prevention and treatment pressure ulcers: a review. J Gerontol A Biod Sci Med Sci 2001;68:704-710, 710-4, 717-722.

61. Lyder CH. Pressure ulcer prevention and management. JAMA 2003;289(2):223-226.

62. Lee VHL, Li SY, Sasaki H, Saettone MF, Chetoni P. Influence of drug release rate on systemic timolol absorption from polymeric ocular inserts in the pigmented rabbits. $J$ Ocular Pharm. 1994;10(2):421-429.

63. Urtti A, Pipkin JD, Rork G, Sendo T, Finne U, Repta AJ. Controlled drug delivery devices for experimental ocular studies with timolol. 2. Ocular and systemic absorption in rabbits. Int J Pharm. 1990;61:241-249.

64. Friedrich SW, Saville BA, Cheng Y-L, Rooman DS. Pharmacokinetic differences between ocular inserts and eyedrops. J Ocular Pharm Ther. 1996;12(1):5-18.

65. Opremcak EM. Uveitis: A Clinical Manual for Ocular Inflammation. NewYork:Springer-Verlag;1995.

66. Nussenblatt RB, Whitcup SM, Palestine AG. Uveitis: Fundamentals and Clinical Practice. St Louis, MI:Mosby;1996.

67. Kunou N, Ogura Y, Honda Y, Hyon S-H, Ikada Y. Biodegradable scleral implant for controlled intraocular delivery of betamethasone phosphate. J Biomed Mater Res. 2000;51:635-641. 
68. DeCampos AM, Sánchez A, Alonso MJ. Chitosan nanoparitcles: a new vehicle for the improvement of the delivery of drugs to the ocular surface. Application to cyclosporin A. Int J Pharm. 2001;224:159-168.

69. Lee Y-C, Millard JW, Negvesky GJ, Butrus SI, Yalkowsky SH. Formulation and in vivo evaluation of ocular insert containing phenylephrine and tropicamide. Int $J$ Pharm. 1999;182:121-126.

70. Holland GN, Pepose JS, Pettit TH, Acquired immunodeficiency syndrome: ocular manifestations. Ophthamology. 1983;90:859-873.

71. Sakurai E, Matsuda Y, Ozeki H, Kunou N, Nakajima K, Ogura Y. Scleral plug of biodegradable polymers containing ganciclovir for experimental cytomegalovirus retinitis. Invest Ophthalmol Vis Sci. 2001;42:2043-2048. 


\section{Chapter II}

\section{Statement of Research Objectives}

The global purpose of this research is to apply a material science based approach to develop a localized delivery system utilizing gelatin as a drug carrier which bioerodes and provides prolonged drug release in a reproducible and predictable fashion.

The main objective of this research is to formulate a gelatin-based matrix, crosslinked using inert, native sugars, fructose and sucrose, that could be utilized for either localized or targeted sustained drug delivery. A material understanding of the gelatin matrix will allow for the development of these matrices into varying geometries, and ultimate use at varying sites of delivery.

To date, aldehydes have been extensively utilized as cross-linking agents; however, they have the potential to cause toxicity and irritation at the delivery site. One specific goal of this work includes evaluating the viability of two sugars (sucrose and fructose) as cross-linking agents of gelatin, replacing the more toxic aldehydes. This will be done by using erosion and thermal studies to assess the structure and strength of the material (materials analysis). Since cross-linking with these sugars is a novel approach, gelatin matrix formulations and processing conditions will be optimized, relating this to the polymer's performance and its physicochemical properties. In order to validate the performance of the new gelatin/sugar matrices, a comparison with a previously established cross-linking agent is necessary. A second goal is to compare and evaluate 
the efficacy of the sugars as cross-linking agents to a more traditional cross-linking agent (formaldehyde).

The matrices possess several complex interrelated properties such as combinations of polymer backbone and cross-linking agent, which can affect swelling and degradation which may affect the release kinetics of drug. Once the material is characterized and optimized, these matrices will be loaded with a prototype drug with an interest in application for drug delivery. The third goal is to characterize the release kinetics and sustained release properties of the optimized gelatin formulations. This will be done using dye indicators as drug prototypes. Dye release will be measured both qualitatively and quantitatively, requiring the development of a direct chemical assays. This data, along with swelling/erosion data, will be used to test models to identify those properties (diffusion, swelling, or erosion) contributing to the drug release. 


\section{Chapter III}

\section{Formulation Development and Optimization}

Through optimization techniques, the gelatin/sugar matrices will be developed to possess a stable, structured matrix demonstrating ideal sustained release delivery characteristics (minimal burst effects, slow erosion, and reproducible sustained release of drug for 12-24 hours). The incorporation of sugar (sucrose and fructose) into gelatin formulations, replacing more toxic aldehydes, should strengthen the material by crosslinking. This can be accomplished by varying the gelatin and sugar concentration across a broad concentration range. Gelatin Type B is the preferred gelatin for localized drug delivery because its isoelectric point $(\mathrm{pI} \sim 5)$ falls closer to the $\mathrm{pH}$ range of physiological conditions when inflammation is present (slightly acidic conditions, with acidity a function of the extent of inflammation). This is important because gelatin swells least at its isoelectric point, which should minimize drug release burst effects and lead to more ideal sustained release characteristics.

\section{Methodology}

\section{Materials}

Gelatin disks were prepared using gelatin Type B 225 Bloom and D(-)-fructose purchased from Sigma (Sigma Chemical Co, St. Louis, MO). D(+)-sucrose was purchased from Fluka Chemical Co., Milwaukee, WI. Ethyl alcohol (absolute) was purchased from Aldrich Chemical Co., Milwaukee, WI. 


\section{Evaluation of Cross-linking Agent - Sugars}

\section{Preparation of non-cross-linked disks}

Gelatin disks were prepared by heating a $15 \% \mathrm{w} / \mathrm{v}$ aqueous gelatin dispersion to $80^{\circ} \mathrm{C}$. The reaction mixture was maintained at $80^{\circ} \mathrm{C}$ for 5 minutes and syringed using a plastic syringe into Teflon coated pans, forming a circular gelatin disk at the bottom of each well. Eight milliliters were syringed into each well, allowing for equal volume measurement of the dispersion for each gelatin disk. The disks were desiccated and allowed to cure either at room temperature or refrigerated for a designated period of time. Disks were determined suitable for handling subsequent to gelation and drying.

\section{Preparation of sugar cross-linked gelatin disks}

The gelatin disks were prepared by adding $1 \mathrm{ml}$ of aqueous sugar solution (containing variable concentrations of fructose or sucrose, ranging from 5 to $50 \%$ ) to $3 \mathrm{ml}$ of a $20 \%$ gelatin solution resulting in a final gelatin concentration of $15 \% \mathrm{w} / \mathrm{v}$. The reaction mixture was heated to and maintained at $80^{\circ} \mathrm{C}$ for 5 minutes. Gelatin matrices were obtained as described above.

\section{Evaluation of the Efficacy of the Sugars}

The evaluation of the efficacy of the simple sugars as cross-linking agents of gelatin and the mechanisms by which this was achieved included the assessment of the strength and structure of the material and relating the polymer's physicochemical properties to performance. The fundamental studies employed fall into two categories and were compared against each other: performance and materials studies. Performance studies include matrix swelling and erosion (under ambient and physiologic conditions) 
and water content (critical since water serves as a plasticizer). Thermal and weight analysis of the samples were used primarily for materials studies.

Indications of the extent of cross-linking, which ultimately influences drug release kinetics, are indirectly measured by the percent of swelling and rate of erosion that the gelatin matrix undergoes when exposed to an aqueous medium subsequent to curing. For most matrix sustained release dosage forms, an ideal swelling and erosion profile shows gradual swelling of the matrix (limited initial burst effect) that plateaus until the matrix erodes away. In theory, films that quickly swell to a high percent of their original weight have the greatest chance of fast, not prolonged, drug release. The same is true for those matrices that erode more quickly.

Thermal analysis has been employed to study the physical nature of the gelatin matrix and its structural thermal transitions as well as the role of water within the gelatin/sugar matrix. At the present time, there are very few techniques available that directly measure the extent of cross-linking within the matrix. The use of Differential Scanning Calorimetry (DSC) allows for the detection of changes in the state of the material as its temperature is increased. The DSC has two pans that sit on a pair of identical platforms connected to a furnace by a common heat flow. The one pan holds the sample and the other is an empty reference pan. The furnace heats the two pans at the same rate. Any changes in the heat capacity are then plotted as the difference in heat flow between the two pans against temperature. 
There are two thermal transitions that can characterize the gelatin films, the glass transition temperature, $T_{g}$, and melting temperature, $T_{m}$. The $T_{m}$ is the temperature at which the crystals within the matrix melt and the matrix becomes a liquid. The $\mathrm{T}_{\mathrm{g}}$ (glass transition temperature) describes the temperature above which an amorphous system is changed from a glassy solid state to a highly viscous liquid state [1]. This is the temperature at which the flexible strands separate and can undergo translational motion. The $T_{m}$ and $T_{g}$ are indirect measures of the extent of cross-linking within the gelatin matrix. The more stable the matrix is, the higher its $\mathrm{T}_{\mathrm{m}}$ and $\mathrm{T}_{\mathrm{g}}$ will be.

\section{Evaluation of film swelling and erosion - Excess moisture, ambient temperature} Under wet conditions, swelling and erosion studies were conducted at room temperature. Dry weights were recorded and each sample was placed in a test tube of distilled water ( $\mathrm{pH} 4.8-5.2)$. On average, dry samples ranged in weight from $100 \mathrm{mg}$ to $200 \mathrm{mg}$. The samples were pulled and weighed at 5, 15, 30 and 300 minutes. The films were weighed every 24 hours until the sample could no longer be weighed. The water in the test tube was changed every 6 days to minimize saturation of the solution with gelatin.

Evaluation of film swelling and erosion - Limited moisture, physiological temperatures The swelling and erosion studies were conducted at $36 \pm 1^{\circ} \mathrm{C}$ in a Boekel Oven (Boekel Scientific, Feastersville, PA). Four samples were cut from each formulation; sample weights were approximately $150 \mathrm{mg}$. The dry weights of samples were recorded and each sample was placed on a nutrient agar plate. The samples were pulled and weighed at 5,15 , and 30 minutes followed by measurements at 30 -minute intervals until 
the sample could no longer be weighed or until a designated time. Zero weight was the point at which the sample was no longer weighable though it may not be fully eroded/dissolved. The percent swelling was calculated based on the following equation: $\%$ Swelling $=\left(\mathrm{m}_{\mathrm{t}}-\mathrm{m}_{\mathrm{i}}\right) / \mathrm{m}_{\mathrm{i}} \times 100$, where $\mathrm{m}_{\mathrm{i}}$ and $\mathrm{m}_{\mathrm{t}}$ are the weights (in $\mathrm{mg}$ ) of the initial sample and at time, $\mathrm{t}$, respectively.

\section{Thermal Characterization}

A piece of the sample was cut before and after drying to be analyzed using a Differential Scanning Calorimeter 7 (DSC) (PerkinElmer Instruments, Norwalk, CT). Four samples from each formulation were analyzed, with the average weight ranging from 0.5 to $2.0 \mathrm{mg}$. The sample was placed into an aluminum sample pan. The pan and sample were covered with a lid that was crimped so that the sample was encapsulated in the pan. The pan with the sample was placed in the sample holder, while an empty reference pan with a cover was placed on the reference side. The study was started after dry nitrogen was passed through the instrument for 10 minutes. A scanning rate of $20^{\circ} \mathrm{C}$ was evaluated in the temperature range of 25 to $300^{\circ} \mathrm{C}$.

\section{Experimental Design for Cross-linking Optimization}

Gelatin matrices were formulated over a wide range of sugar concentrations, 5 to $50 \%$, and performance was evaluated in order to determine the optimal concentrations of the sugars. The optimal concentration is a concentration in which maximum crosslinking between the gelatin and the cross-linking agent occurs, leading to a matrix with relatively low swelling and prolonged erosion rates. Swelling and erosion studies were conducted to compare gelatin to equal molar gelatin/sugar formulations as well as equal 
concentration gelatin/sugar matrices. Equal molar gelatin/sugar formulations were evaluated in order to determine which sugar is the most efficient cross-linking agent. Thermal analysis studies were conducted to confirm strength and stability of the matrices.

\section{Evaluation of Excipients - Dispersion Agent}

While initial formulations utilized water as the dispersion agent in making the gelatin disks, a sub-set of studies were performed to assess the influence of ethanol on film formation. Ethanol appeared to have the advantage of drying the matrices to a greater extent, increasing solubility for water insoluble drugs, and increasing the permeability across membranes.

Preparation of gelatin disks using ethanol

Gelatin disks with no cross-linking agent were prepared by heating a $15 \% \mathrm{w} / \mathrm{v}$ gelatin solution of varying ratios (ranging from 50 to $100 \%$ ) of ethanol and water to $80^{\circ} \mathrm{C}$ for 5 minutes. The gelatin solution was syringed using a syringe into Teflon coated pans, forming a circular gelatin disk. The disks were desiccated either at room temperature or refrigerated for a designated period of time.

\section{Preparation of sugar cross-linked gelatin disks using ethanol}

The gelatin disks were prepared by adding a $1 \mathrm{ml}$ aqueous solution containing $632.5 \mathrm{mg}$ of fructose or $1200 \mathrm{mg}$ of sucrose to $3 \mathrm{ml}$ of a $20 \%$ gelatin solution (resulting in a final gelatin solution of $15 \% \mathrm{w} / \mathrm{v}$ ) of varying ratios of ethanol and water. The reaction mixture was heated to $80^{\circ} \mathrm{C}$ and maintained at that temperature for 5 minutes. Gelatin matrices were obtained as described above. 


\section{Evaluation of the Efficacy of the Dispersion Agent}

Matrices made with ethanol were evaluated using the same methods as those used on aqueous-based matrices. See Part II of this chapter ("Evaluation of the Efficacy of the Sugars") for a description of these methods.

\section{Experimental Design for Excipient Optimization}

Gelatin matrices were formulated over a range of alcohol concentrations, 30 to $100 \%$, and performance was evaluated in order to determine the role of water within the matrix. Swelling and erosion studies were conducted to compare gelatin/water formulations to gelatin/ethanol formulations. Thermal analysis studies were conducted to confirm strength and stability of the matrices, and identify any change in the materials structure properties.

\section{Results}

To date, there have been several proposed mechanisms of gelatin cross-linking but no direct methods in which to measure the extent of the cross-linking. Digenis, et al [2] proposed several mechanisms in which all of the reactions involve $\varepsilon$-amino groups of lysine. Bubnis et al [3] modified a previous assay utilizing 2, 4, 6 - trinitrobenzenesulfonic acid (TBNS) to determine the concentration of $\varepsilon$-amino groups. This assay is a step toward defining the extent of cross-linking, but it has limitations. The assay provides a reliable measure of the $\varepsilon$-amino groups; however, it is limited to a small range of sample weights as it is dependent on the number of $\varepsilon$-amino groups per molecule of protein. 
Indications of the extent of cross-linking are the percent swelling and rate of erosion that the gelatin matrix undergoes when exposed to an aqueous medium subsequent to curing. Low percent swelling and prolonged erosion would be indicative of matrix strengthening via cross-linking. These should be supported through shifts in the material's thermal profile (change in matrix melting and glass transition temperatures). Matrices that rapidly swell to a high percent of their original weight have the greatest chance of fast, not prolonged, drug release. The same is true for those matrices that erode more quickly. For most forms of sustained release, an ideal swelling and erosion profile shows gradual swelling of the matrix that plateaus until the matrix erodes away, with the matrix remaining relatively intact throughout the desired drug release time frame.

\section{Visual Observations}

After the gelatin disks were formed, notable differences were seen in the physical nature of the non-cross-linked and cross-linked disks. The circular disks had a diameter of $5.3 \mathrm{~cm}$ and a thickness of $2-3 \mathrm{~mm}$. The disks were transparent with a tint of yellowbrown. Upon gelation, the disks are jelly-like and rich in water. As curing occurs, the disks undergo syneresis. Syneresis is the natural shrinkage of the gelatin disks due to the continued coarsening of the matrix or fibrous structure of the gel with a consequent squeezing-out effect [4]. The non-cross-linked disks become extremely dry and brittle over time. The gelatin/sugar disks become dry, but remain flexible and pliable. The noncross-linked gelatin matrices offered the greatest resistance when cutting them into smaller samples. The gelatin/sugar matrices had a lower resistance due to the sugar's hygroscopic properties with fructose appearing to retain more water than sucrose. 


\section{Swelling and Erosion}

The swelling and erosion studies compared gelatin and gelatin/sugar formulations. In theory, during the initial phase of the swelling process, it is expected that the matrix will absorb water, disrupting the non-covalent interactions on the outside of the matrix. Thus, the swelling/erosion profile would show an initial rise in the percent swelling. The swelling process would continue as the solvent continues to proceed within the matrix, causing the free strands to become mobile, leaving the strong covalent bonds intact, and starting the erosion process. Over time, the gelatin matrix should reach an equilibrium in which the swelling stops or swelling and erosion rates are equal. The swelling/erosion profile should show a plateau that may last for a few minutes or many hours. Eventually, the matrix becomes extremely disentangled, stressing the covalent bonds and causing them to break, leading to the disintegration and dissolution of the matrix. This is seen as a rapid decrease, tailing curve at the end of the swelling/erosion profile.

As demonstrated by the swelling and erosion data, reported in Figure 6, the incorporation of equal molar concentrations of sugar, with a final concentration of $30 \%$ for sucrose or $15.8 \%$ for fructose, decreased the extent of swelling in both formulations. (NOTE: The last point on all swelling/erosion profiles represents the last data point in which $50 \%$ of the samples were weighable.) Table 1 shows the initial dry weights of all four formulations. The matrices with no cross-linking agent exhibit a large burst with maximum swelling of 3.5 times its initial weight compared to a 2 -fold increase in the cross-linked matrices. The gelatin and gelatin/sucrose matrices have a similar overall profile in which the swelling occurs during the first 4 hours, followed by a short 


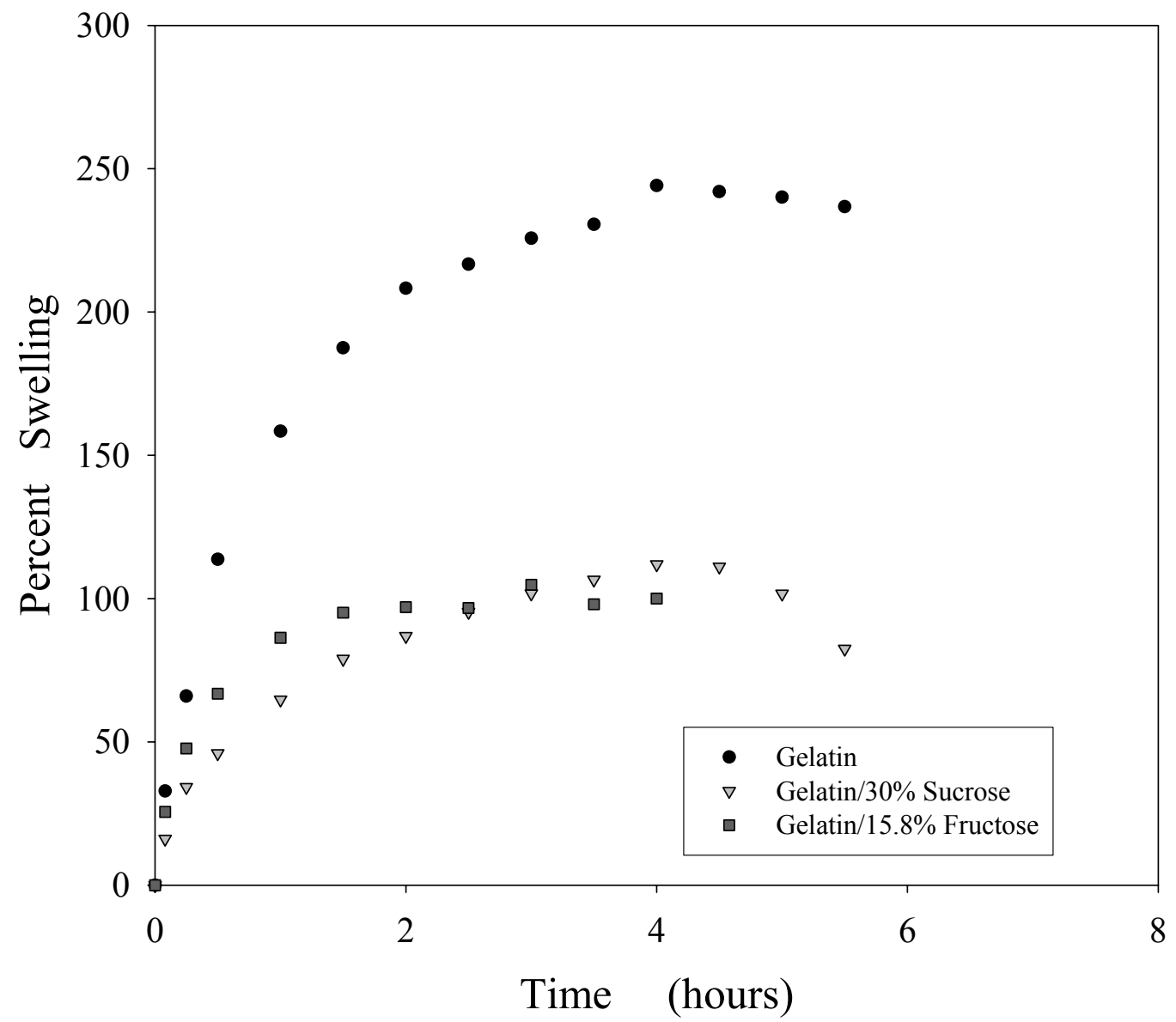

Figure 6. Swelling and erosion profiles comparing gelatin and equal molar gelatin $/ \mathbf{3 0} \%$ sucrose, and gelatin $/ \mathbf{1 5 . 8} \%$ fructose matrices.

Matrices were cured for 3 days at refrigerated temperatures and evaluated under limited moisture and physiological temperatures. The results are plotted as an average of triplicate measurements. 
Table 1. A comparison of the initial weights for the gelatin, gelatin/30\% sucrose and gelatin $/ \mathbf{1 5 . 8 \%}$ fructose matrices.

Matrices were cured for 3 days at refrigerated temperatures. Data represents a mean of 3 determinations.

\begin{tabular}{|c|c|}
\hline & $\begin{array}{c}\text { Weight } \\
(\mathrm{mg} \pm \mathrm{SD})\end{array}$ \\
\hline Gelatin & $74.15 \pm 3.25$ \\
\hline Gelatin/30\% Sucrose & $75.53 \pm 4.56$ \\
\hline Gelatin/15.8\% Fructose & $72.18 \pm 1.26$ \\
\hline
\end{tabular}


equilibrium and rapid erosion. The gelatin/fructose matrices undergo a shorter swelling period ( $\sim 1.5$ hours) followed by a 2.5 hour equilibrium and fast erosion. As the swelling process occurs, the variability between samples is small, ultimately increasing as the matrices begin the erosion process (Table 2). The increase in variability may be due to the swelling of the matrices causing the gelatin strands to become mobile and/or increased matrix erosion. The increased mobility allows the water to have greater access to the tight crystallite areas causing variable disentangling and erosion rates.

In comparing the gelatin/sugar matrices at equal concentrations (30\% final sugar concentration), both matrices demonstrate a low percent swelling with the gelatin/fructose swelling to 1.5 times its original weight (Figure 7). As seen before, the gelatin/sucrose matrices demonstrated slow swelling followed by rapid erosion. The gelatin/fructose matrices demonstrated rapid swelling followed by rapid erosion. Neither of the formulations reached the desired plateau where the swelling and erosion rates appear to be equal.

Gelatin/sugar matrices were formulated over a wide range of concentrations, 5 to $50 \%$ final sugar concentration, and performance was evaluated in order to determine the optimal concentration. At low sugar concentrations, the gelatin/sugar matrices were physically similar to the gelatin matrices (extremely brittle upon visual examination). The gelatin/sugar matrices at high sugar concentrations were difficult to formulate and dry due to the presence of excess sugar. The films were extremely wet and tacky after a 24 hour curing process and did not form a firm jelly-like structure until later in the curing 
Table 2. Comparison of the variability of swelling profiles.

Matrices were cured for 3 days at refrigerated temperatures and evaluated under limited conditions and physiological temperatures. Data represents a mean of 3 determinations.

\begin{tabular}{|c|c|c|c|c|c|c|}
\hline Hours & $\begin{array}{c}\text { Gelatin } \\
(\mathrm{mg} \pm \mathrm{SD})\end{array}$ & $\begin{array}{c}\text { Standard } \\
\text { Error }\end{array}$ & $\begin{array}{c}\text { Gealtin/Sucrose } \\
(\mathrm{mg} \pm \mathrm{SD})\end{array}$ & $\begin{array}{c}\text { Standard } \\
\text { Error }\end{array}$ & $\begin{array}{c}\text { Gelatin/Fructose } \\
(\mathrm{mg} \pm \mathrm{SD})\end{array}$ & $\begin{array}{c}\text { Standard } \\
\text { Error }\end{array}$ \\
\hline 0 & 0.00 & & 0.00 & & 0.00 & \\
\hline 0.08 & $32.88 \pm 2.20$ & 6.69 & $16.19 \pm 3.57$ & 22.07 & $25.56 \pm 3.21$ & 12.56 \\
\hline 0.25 & $66.00 \pm 3.66$ & 5.55 & $34.19 \pm 3.37$ & 9.85 & $47.73 \pm 2.96$ & 6.21 \\
\hline 0.50 & $113.71 \pm 5.87$ & 5.16 & $45.97 \pm 3.66$ & 7.96 & $66.81 \pm 4.51$ & 6.76 \\
\hline 1.00 & $158.41 \pm 3.75$ & 2.37 & $64.72 \pm 4.85$ & 7.49 & $86.35 \pm 11.11$ & 12.87 \\
\hline 1.50 & $187.47 \pm 4.30$ & 2.29 & $78.93 \pm 5.31$ & 6.73 & $95.08 \pm 10.30$ & 10.83 \\
\hline 2.00 & $208.26 \pm 5.05$ & 2.42 & $86.86 \pm 5.31$ & 6.12 & $97.03 \pm 21.68$ & 22.34 \\
\hline 2.50 & $216.69 \pm 10.67$ & 4.92 & $95.34 \pm 4.05$ & 5.30 & $96.74 \pm 21.10$ & 21.81 \\
\hline 3.00 & $225.71 \pm 21.24$ & 9.41 & $101.74 \pm 4.02$ & 3.96 & $104.84 \pm 19.14$ & 18.26 \\
\hline 3.50 & $230.61 \pm 15.14$ & 6.57 & $106.52 \pm 4.95$ & 4.65 & $98.05 \pm 25.16$ & 25.66 \\
\hline 4.00 & $244.11 \pm 15.81$ & 6.48 & $111.93 \pm 6.09$ & 5.44 & $100.03 \pm 29.67$ & 29.66 \\
\hline 4.50 & $241.96 \pm 23.13$ & 9.56 & $111.31 \pm 9.14$ & 8.23 & & \\
\hline 5.00 & $240.09 \pm 32.13$ & 13.38 & $101.66 \pm 18.10$ & 17.80 & & \\
\hline 5.50 & $236.72 \pm 43.28$ & 18.28 & $82.42 \pm 36.18$ & 43.90 & & \\
\hline
\end{tabular}




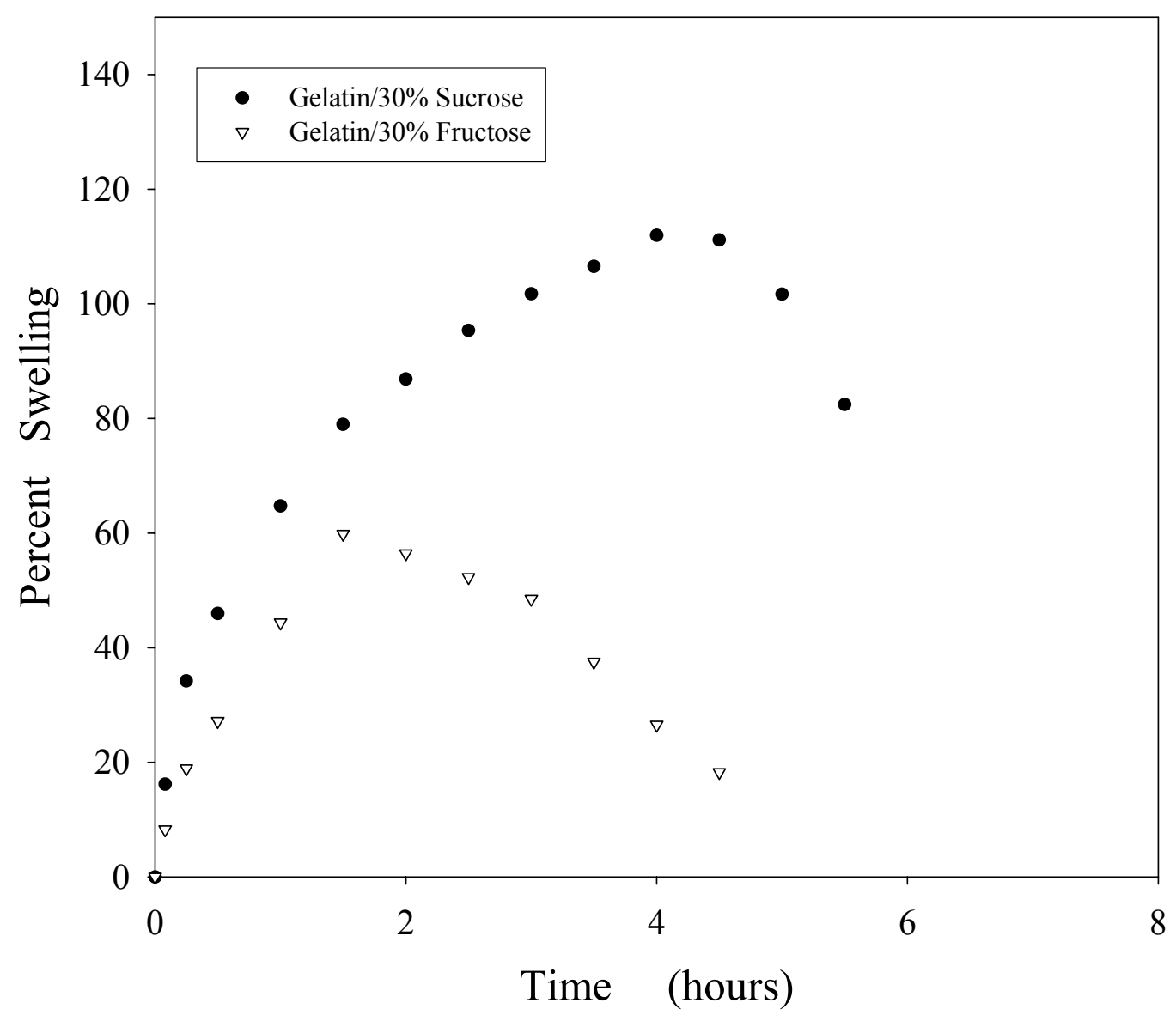

Figure 7. Swelling and erosion profiles comparing equal concentrations of gelatin $/ \mathbf{3 0} \%$ sucrose and gelatin $/ \mathbf{3 0} \%$ fructose matrices.

Matrices were cured for 3 days at refrigerated temperatures and evaluated under limited moisture and physiological temperatures. The results are plotted as an average of triplicate measurements. 
process. At the highest concentration of sugar, the gelatin disks took longer to gel than any other concentration (120 minutes versus 30 minutes). This is most likely due to the sugar's hygroscopic properties that cause the sugar to retain water, leaving the matrices sticky, less defined in structure, and difficult to handle.

The low concentration gelatin/sucrose matrices demonstrated quick swelling and erosion within the first 2 hours without establishing a classic swelling/erosion profile (Figure 8). The high concentration gelatin/sucrose matrices eroded within 4 hours exhibiting a low percent swelling (less than 50\%). Even though the gelatin/sucrose matrices at 30\% demonstrated a high percent swelling, the overall performance was better than the other formulations because of the longer, more desirable swelling/erosion profile.

The gelatin/fructose matrices performed well at or above the $15 \%$ concentration range (Figure 9). The higher concentrations demonstrated a low percent swelling and a similar erosion time as the $15 \%$ fructose formulation. The $15 \%$ formulation exhibited the best profile because it establishes a plateau that lasts for 2.5 hours. Visual observations of the swelling and erosion process indicate that, at the extreme concentrations, the gelatin/sugar matrices are unable to maintain the physical integrity of the matrix. At low concentrations, the films became swollen and lose shape within the first 2 hours of being exposed to the agar plates. The high concentration matrices did not become as swollen but began to lose their shape around 2 hours. The intermediate concentration matrices maintain their physical integrity throughout the initial swelling as well as the plateau 


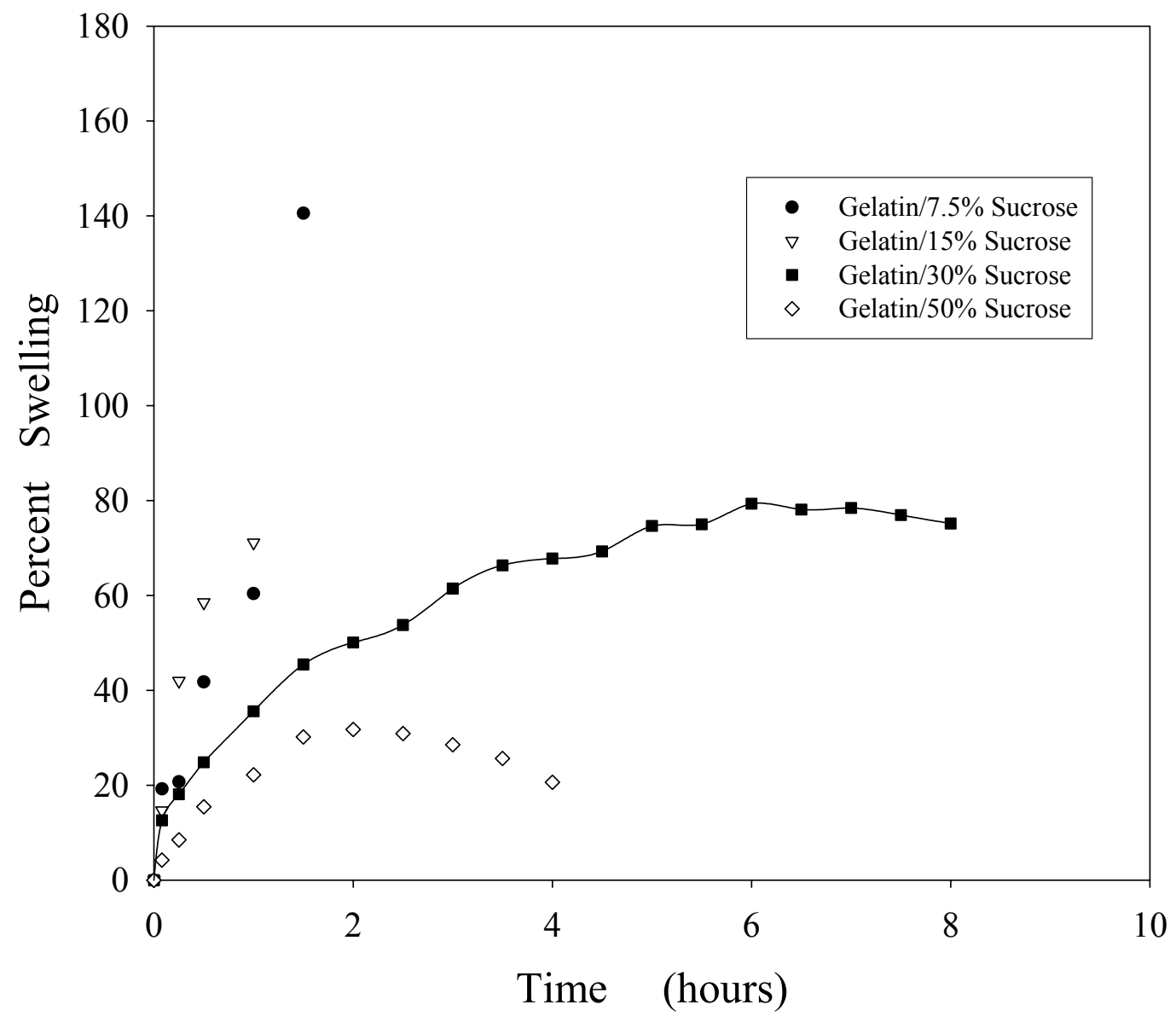

Figure 8. Swelling and erosion profile of varying concentrations of sucrose in gelatin/sucrose matrices.

Matrices were cured for 3 days at refrigerated temperatures and evaluated under limited moisture and physiological temperatures. The results are plotted as an average of triplicate measurements. 


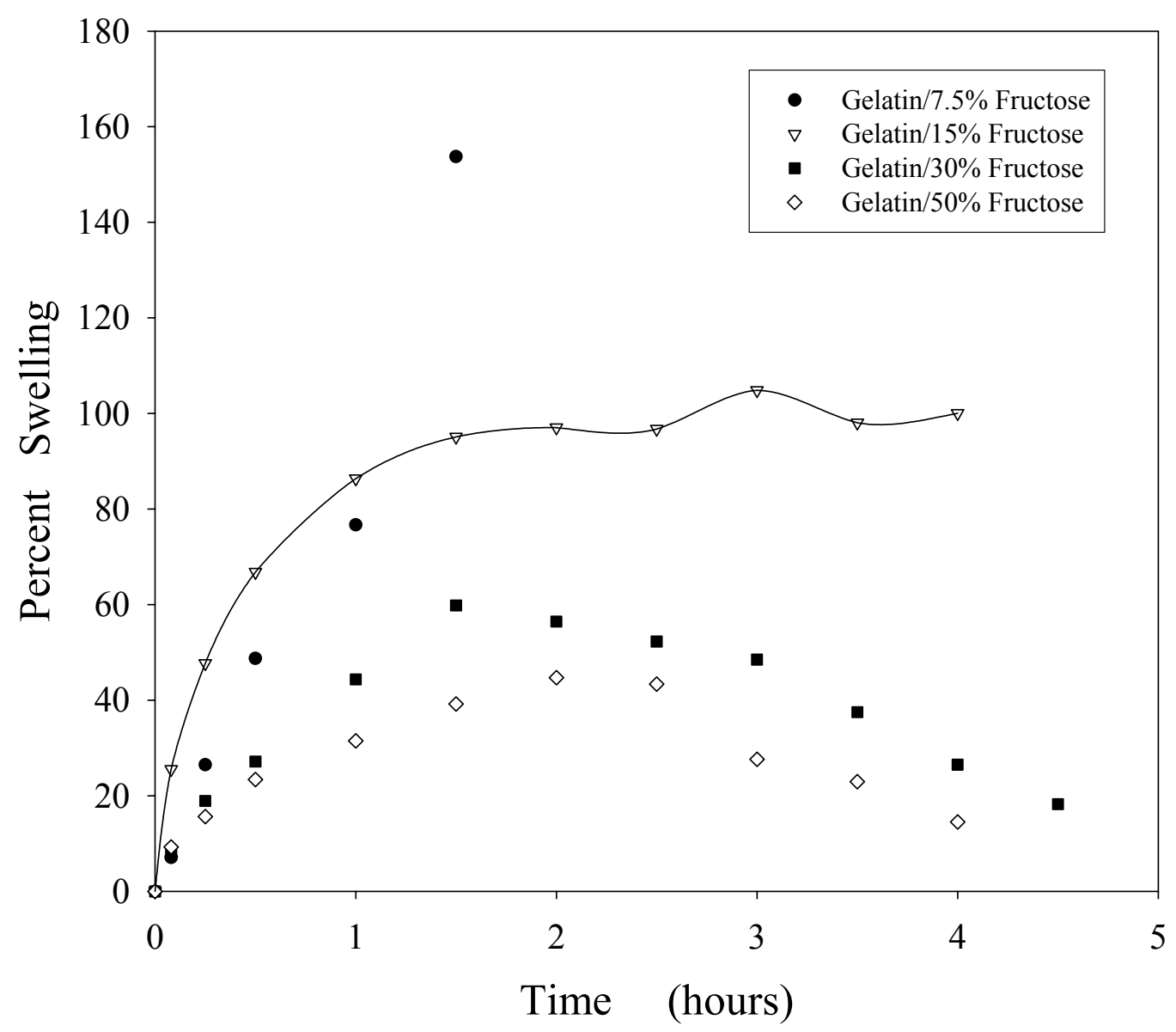

Figure 9. Swelling and erosion profile of varying concentrations of fructose in gelatin/fructose matrices.

Matrices were cured for 3 days at refrigerated temperatures and evaluated under limited moisture and physiological temperatures. The results are plotted as an average of triplicate measurements. 
phase. As the erosion process takes over, the gelatin/sugar matrices dissolve over the next several hours. Based on these observations, the matrices with a prolonged plateau and short erosion were selected as optimized formulations. These may demonstrate a more prolonged and predictable drug release pattern than matrices with limited plateaus and prolonged erosion phases.

\section{Thermal Analysis}

Thermal analysis data shows three endothermic peaks in the gelatin matrix spectrums (Figure 10). A peak between $90-100^{\circ} \mathrm{C}$ was seen indicating the presence of excess water within the matrix. As the matrix dries, this peak diminishes and eventually disappears after 3 days (data not shown). A second peak appears between $160-220^{\circ} \mathrm{C}$, representing the gelatin or gelatin/sugar matrix melting temperature, the transition to a liquid state. A distinct and representative glass transition peak could not be identified in this data. At room temperature, the gelatin/sugar matrices are soft and pliable indicating that the matrices are above the glass transition. Significantly, the gelatin/sugar peaks are shifted to slightly higher temperatures compared to the non-cross-linked gelatin matrices, suggesting a more ordered structure and stability of the cross-linked matrices. The third peak is a small peak around $240-260^{\circ} \mathrm{C}$, caused by the charring of excess sugar.

The gelatin/sugar matrices did not exhibit the same water peak around $100^{\circ} \mathrm{C}$ as seen with the gelatin matrices. This may be due to the hygroscopic property of the sugars causing the water to be more tightly bound within the matrix. The non-cross-linked matrix does not hold the water as tightly within the matrix, leading to the evaporation of water (boiling point of water is $100^{\circ} \mathrm{C}$ ) upon heating the matrix. The gelatin/sucrose 


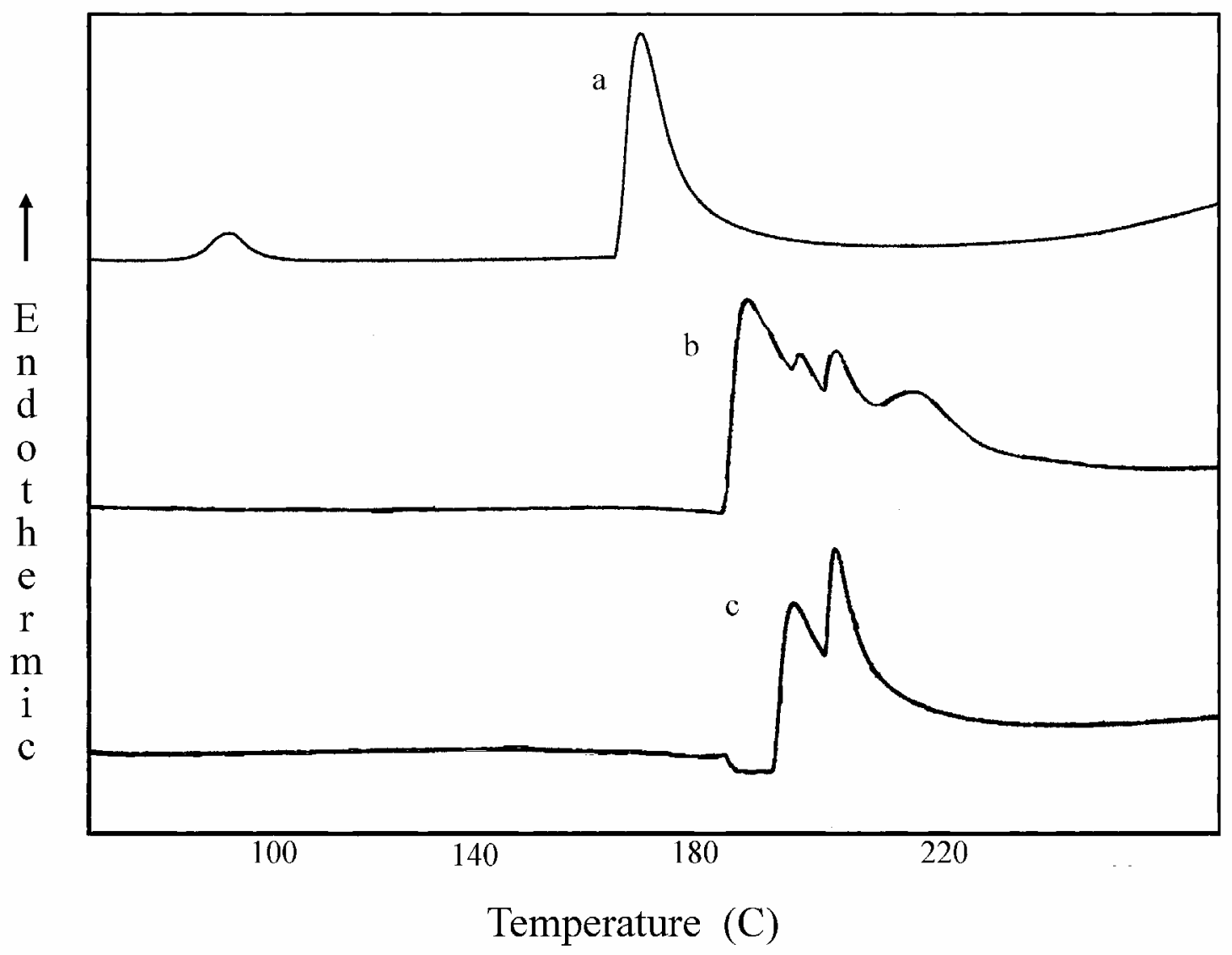

Figure 10. Thermal analysis scans of the gelatin (a), gelatin/30\% sucrose (b), and gelatin $\mathbf{1 5 . 8 \%}$ fructose (c) matrices.

Matrices were cured for 3 days at refrigerated temperatures. 
matrices show a multiple peak melting temperature in the range of $170-220^{\circ} \mathrm{C}$ that correlates with the matrix melting along with, possibly, the melting of excess sucrose within the matrix (Figure 11). Multiple peaks within the $\mathrm{T}_{\mathrm{m}}$ range are seen with the gelatin/fructose matrix which may be due to a limited amount of excess fructose in the matrix (Figure 12). The incorporation of the fructose into the gelatin matrix causes a decrease in the melting temperature of pure fructose.

It would be expected that, like most polymers, the gelatin matrices would show evidence of a glass transition temperature. For polymer matrices, in theory, when the matrix reaches the glass transition temperature, the strands have gained enough energy to move into very ordered arrangements or crystals. As the strands form crystallites, they give off heat which is seen as a slight exothermic change in the baseline. At room temperature, the gelatin/sugar matrices are plasticized (and beyond the glass transition temperature), thus no exothermic event would be expected from the gelatin/sugar matrices. However, the non-cross-linked gelatin matrices are brittle in nature and a glass transition would be expected prior to the melting temperature. While some baseline changes were observed for the matrix formulations prior to the melting of the matrix, no distinct exothermic peak was observed and, thus, $\mathrm{T}_{\mathrm{g}}$ could not be consistently identified for that formulation. Therefore, no $\mathrm{T}_{\mathrm{g}}$ is reported in these results for non-cross-linked matrices. More extensive studies by Kouassi, et al [5] have shown similar results. Kouassi, et al investigated the effects of water on the hydrolysis of sucrose by invertase. The authors found that it was difficult to determine the glass transition temperature due to the interference from crystallization during the storage process. This was in agreement 


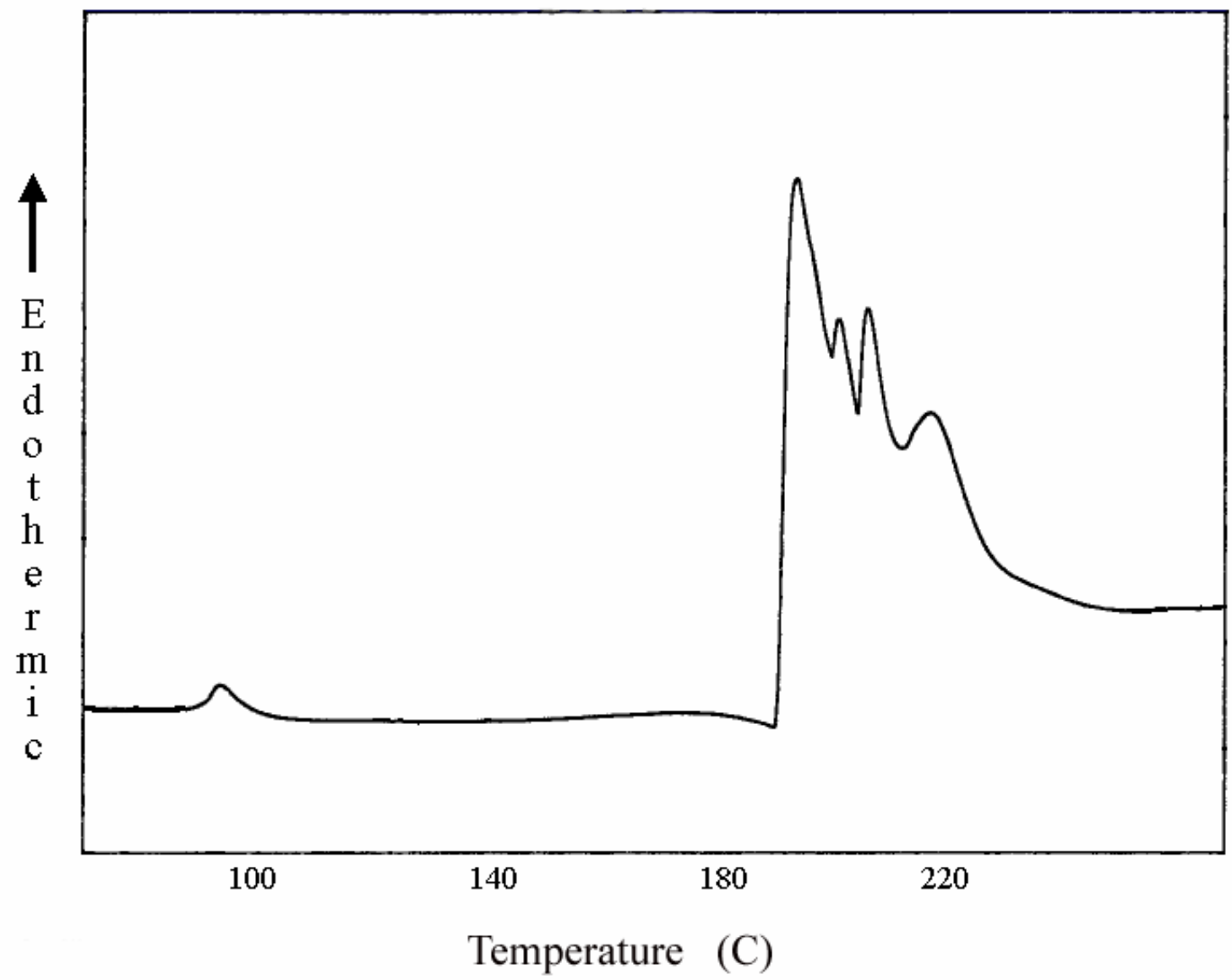

Figure 11. Thermal analysis scan of the melting temperature of sucrose. 


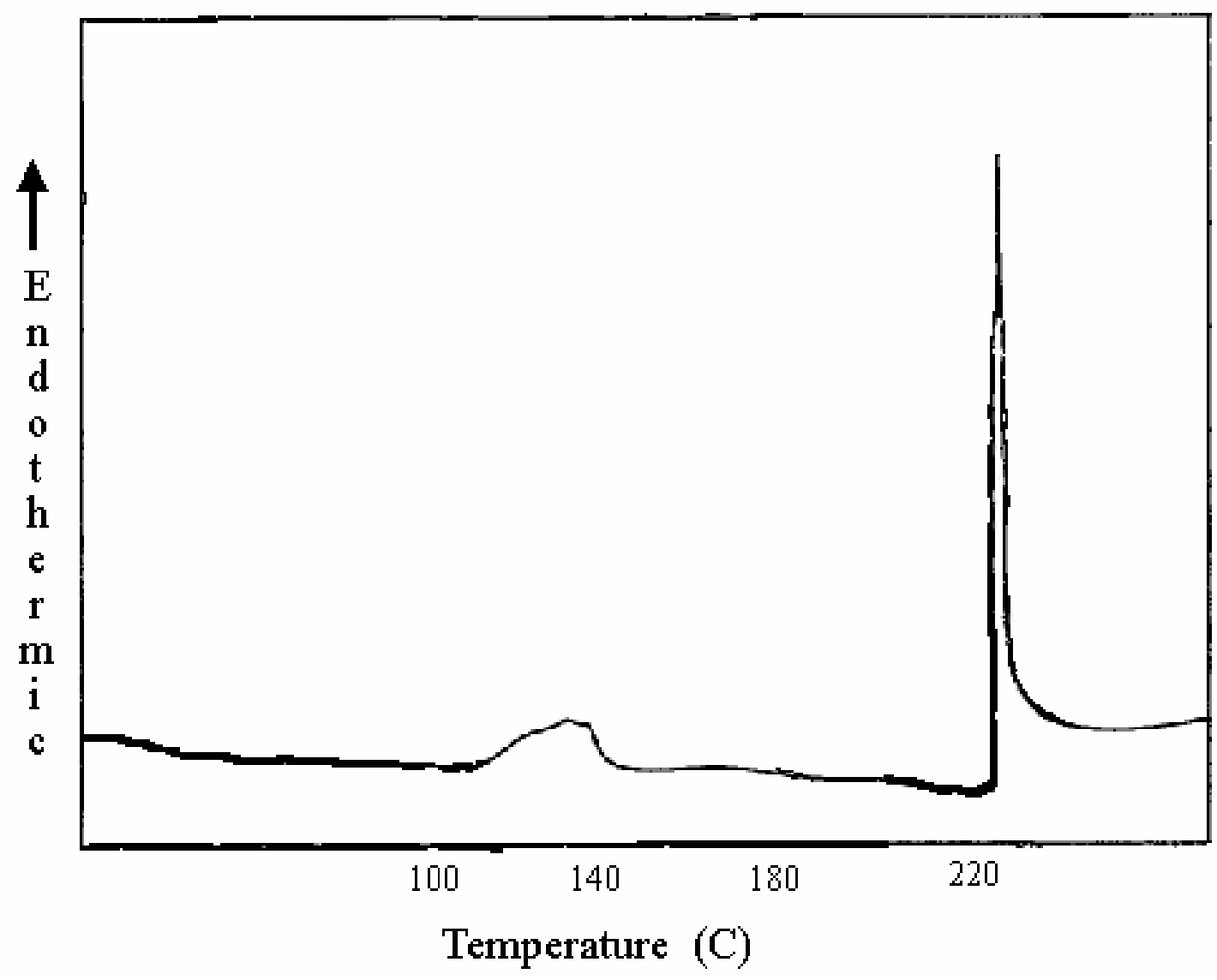

Figure 12. Thermal analysis scan of the melting temperature of fructose. 
with Hagiwara, et al [6] who reported that crystallization took place during the storage of samples.

\section{Influence of Excipients}

The physical appearance of the matrices changed when the aqueous medium was replaced with varying concentrations of ethanol. After 24 hours of curing under refrigerated temperatures, the gelatin/sugar formulations exhibited a cream color that started at the edges of the circular disk and slowly spread throughout the disk until the film became translucent. As the curing and maturation process continued, in which the matrices undergo syneresis (forming additional contact points between the gelatin strands as well as additional crystallites [4]), the gelatin/sucrose disks retained the cream color. A transparent ring around the edge of the gelatin/fructose disks appeared and continued to grow in size as the disks were allowed to further cure for several months. The gelatin disks were hazy but remained transparent throughout the curing process. As the disks became dry, the gelatin disks started to turn in and curl into a roll. The dryness of the three formulations was drastically different. The gelatin disks were extremely dry and brittle. Eventually, the disks became so inflexible that they could not be used in future studies. The gelatin/sucrose disks were dry with an uneven, rough texture that was pliable enough to cut samples. After months of curing at room temperatures, the disks became extremely dry and sucrose precipitated out of the disk. The gelatin/fructose disks were extremely pliable with a rubbery, plastic-like texture. These disks were tacky and had a slight scent of ethanol to them. 
The gelatin/ethanol/water matrices did not perform well in the swelling and erosion studies. The ethanol-based matrices eroded over a shorter period of time than the water-based matrices (Figure 13). This may be due to the ability of ethanol to denature the gelatin strands. Some studies have reported the use of organic solvents, particularly ethanol, to produce gelatin coacervates as a technique to separate gelatin into fractions with different molecular weight distributions and averages [7]. Under normal conditions, the long strands of gelatin are cross-linked via inter-and intrastrand linkages. These strands can also become entangled or wrapped around each other, leading to increased strength and structure of the matrix. However, in the ethanol-based formulation, the gelatin strands may not undergo the extensive entanglement process due to the shorter chains, causing the matrix to lose strength. As the swelling and erosion process occurs, the smaller untangled strands of gelatin are more mobile and erode away, ultimately, enhancing the erosion rate. In theory, this may contribute to the fluctuations seen in the swelling profile (Figure 13). This may lead to quick erosion exposing a dry inner core to the medium causing a quick swelling burst.

A comparison of the melting temperature of the water-based (Figure 10) and ethanol-based (Figure 14) gelatin formulations show that the ethanol-based formulation is a less stable matrix. Table 2 shows a pronounced shift to lower melting temperatures for the ethanol-based matrices suggesting that the ethanol creates a less stable matrix. It also appears from these profiles, that the addition of the sugars to the ethanol-based formulations for cross-linking had little affect on strengthening the matrices, as the $\mathrm{T}_{\mathrm{m}}$ of the sugar-based matrices changed very little, only broadening. 


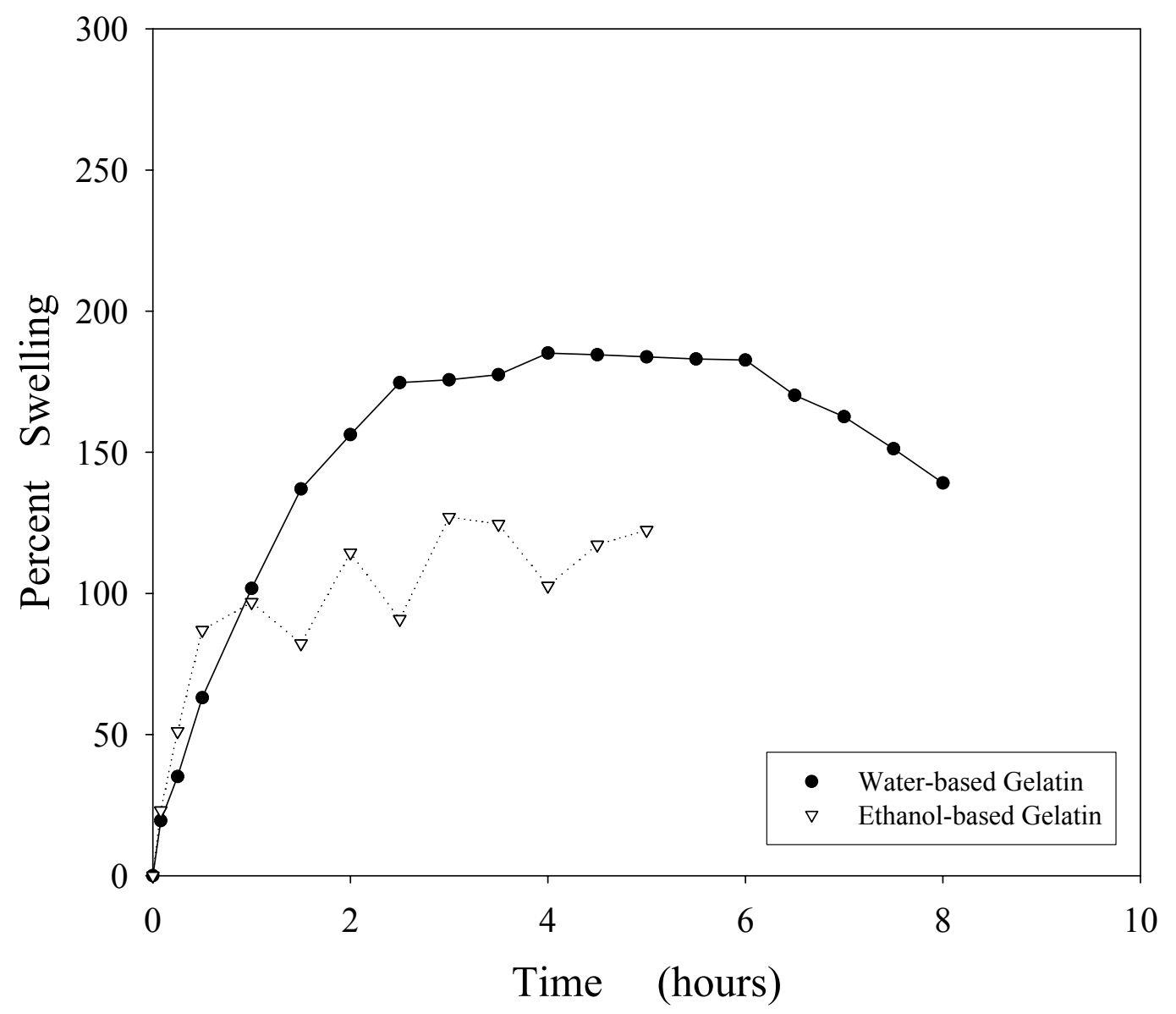

Figure 13. Swelling and erosion profiles of water-based gelatin and ethanol-based gelatin matrices.

Matrices were cured for 3 days at refrigerated temperatures and evaluated under limited moisture and physiological temperatures. The results are plotted as an average of triplicate measurements. 


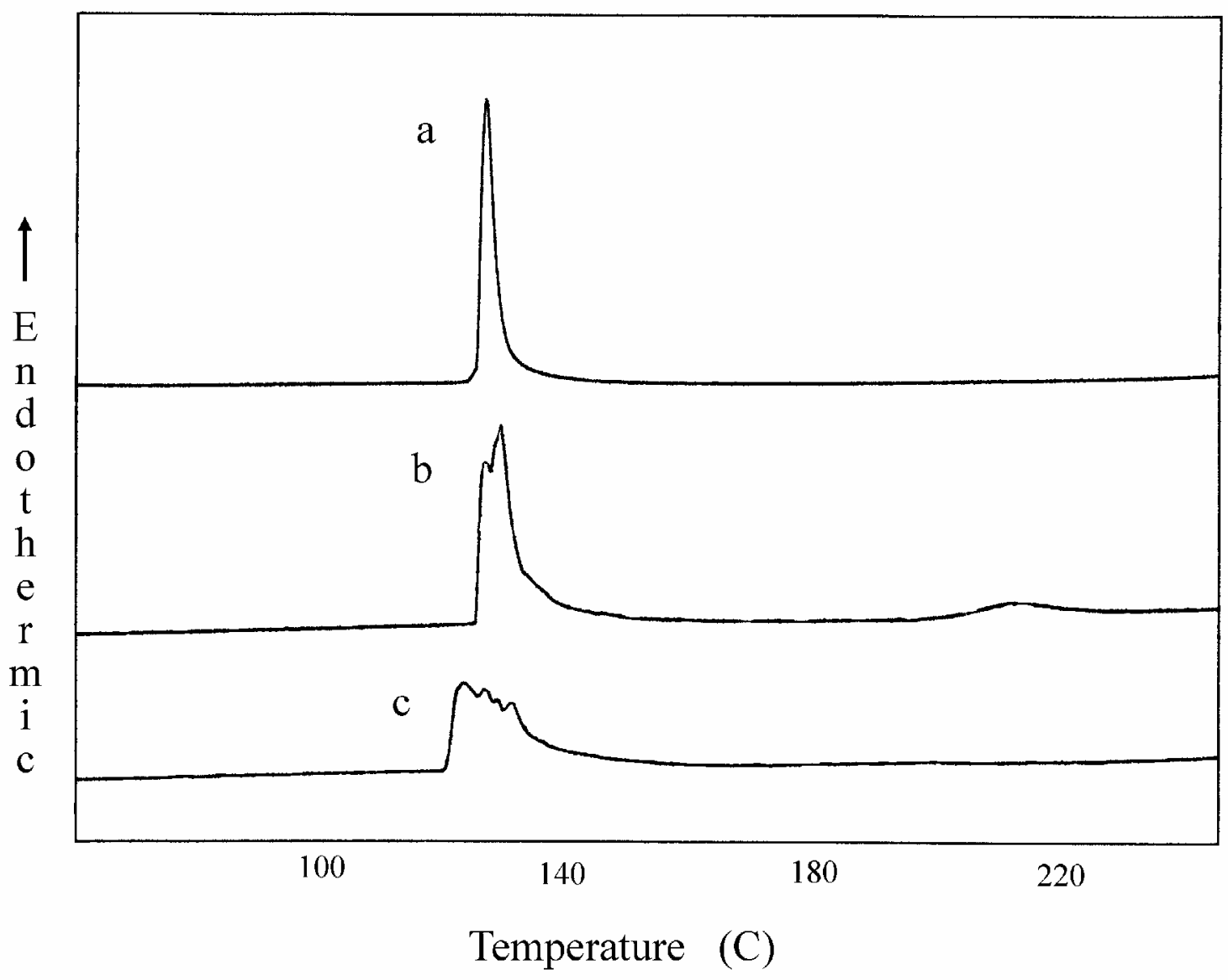

Figure 14. Thermal analysis scans of ethanol-based gelatin (a), gelatin/30\% sucrose (b), and gelatin $/ \mathbf{1 5 . 8 \%}$ fructose (c) matrices.

Matrices were cured for 3 days at refrigerated temperatures and evaluated under limited moisture and physiological temperatures. 
Unlike the water-based formulations, the melting temperatures of the ethanolbased formulations do not have the characteristic shift to the right as the matrix dries and cures (Table 3). This may be due to the volatile properties of ethanol. Early in the gelation process, the ethanol evaporates out of the disks causing the disks to be 'frozen' in time. This may not allow the gelatin strands to be mobile and move into closer proximity with each other during curing, leaving a limited number of strong covalent bonds present in the matrix, as well as limiting strand entanglement. The lack of covalent bonds does not allow the disks to shrink and form the essential hydrogen bonds to enhance the strength, stability, and ordered structure of the matrix. The energy required to melt the ethanol-based formulations is much lower than the water-based formulations. This may be due to limited covalent and hydrogen bonds within the matrix, allowing the shorter gelatin strands to more readily become mobile and melt. A decrease in the area under the curve is also seen over the 5 day curing process. This is likely due to the excess amount of water present in the matrices in the early part of the curing process. In the early stages of the curing process, there are two events occurring during the melting phase, evaporation of excess water and melting of the matrix. At the end of the curing process, most of the water is driven out of the matrix and the resulting area is due mainly to the energy required to melt the polymer matrix. 
Table 3. Comparison of $T_{m}$ and peak area of the gelatin/water and gelatin/70\% ethanol/30\% water formulations before and after vacuum drying for $\mathbf{3 0}$ minutes.

The matrices were cured over a 5 day refrigeration period. Data represents a mean of 3 determinations.

\begin{tabular}{|c|c|c|}
\hline $\begin{array}{c}\text { Pre-dry } \\
\text { Film }\end{array}$ & $\begin{array}{c}\text { Temperature } \\
\left({ }^{\circ} \mathrm{C}\right)\end{array}$ & $\begin{array}{c}\text { Area } \\
(\mathrm{J} / \mathrm{g})\end{array}$ \\
\hline $\begin{array}{c}\text { Water } \\
\text { Day 1 }\end{array}$ & 156 & 1112 \\
\hline $\begin{array}{c}\text { EtOH } \\
\text { Day 1 }\end{array}$ & 133 & 833 \\
\hline & 173 & 375 \\
\hline $\begin{array}{c}\text { Water } \\
\text { Day 3 }\end{array}$ & 143 & 143 \\
\hline $\begin{array}{l}\text { EtOH } \\
\text { Day 3 }\end{array}$ & 174 & 270 \\
\hline $\begin{array}{l}\text { Water } \\
\text { Day 5 }\end{array}$ & 142 & 175 \\
\hline $\begin{array}{l}\text { EtOH } \\
\text { Day 5 }\end{array}$ & & \\
\hline
\end{tabular}

\begin{tabular}{|c|c|c|}
\hline $\begin{array}{c}\text { Post-dry } \\
\text { Film }\end{array}$ & $\begin{array}{c}\text { Temperature } \\
\left({ }^{\circ} \mathrm{C}\right)\end{array}$ & $\begin{array}{c}\text { Area } \\
(\mathrm{J} / \mathrm{g})\end{array}$ \\
\hline $\begin{array}{c}\text { Water } \\
\text { Day 1 }\end{array}$ & 154 & 669 \\
\hline $\begin{array}{l}\text { EtOH } \\
\text { Day 1 }\end{array}$ & 130 & 261 \\
\hline & 182 & 209 \\
\hline $\begin{array}{l}\text { Water } \\
\text { Day 3 }\end{array}$ & 145 & 172 \\
\hline $\begin{array}{l}\text { EtOH } \\
\text { Day 3 }\end{array}$ & 193 & 187 \\
\hline $\begin{array}{l}\text { Water } \\
\text { Day 5 }\end{array}$ & 147 & 141 \\
\hline $\begin{array}{l}\text { EtOH } \\
\text { Day 5 }\end{array}$ & & \\
\hline
\end{tabular}




\section{Discussion}

When comparing the three formulations, the gelatin/sucrose matrices show better swelling and erosion characteristics for sustained release delivery, lasting 30\% longer than the gelatin/fructose matrices and swelling by $20 \%$ of their initial dry weight. Per mole, sucrose also appears to be a more effective cross-linking agent than fructose. The ideal swelling and erosion profile includes a slow initial swelling followed by a prolonged plateau before erosion begins. At this point in the research (Phase I), the sucrose-based formulations best matched these performance criteria.

Within each formulation type, the gelatin/sugar matrix demonstrating the most acceptable swelling/erosion performance were the matrices with final concentrations of $30 \%$ for the sucrose and $15.8 \%$ for the fructose. These formulations show significant improvement in swelling/erosion behavior in comparison with non-cross-linking gelatin matrices.

In an attempt to decrease the amount of water used in the dispersion and casting of the gelatin matrices, so as to minimize water's plasticizing affect on the matrix, ethanol was used to substitute a percentage of water. However, use of ethanol as a dispersion agent proved no benefit, as the films eroded more quickly and gave an erratic swelling phase.

Finally, evidence of the affect of sugars on the cross-linking and increased structure and stability of the matrices was supported through thermal analysis. Matrices 
cross-linked with sugars remained intact longer and melted at a higher $\mathrm{T}_{\mathrm{m}}$ than those with no cross-linking agent.

Based on these results, matrices for future studies in this work were developed using the following optimized formulations: $15 \%$ gelatin, $15 \%$ gelatin $/ 30 \%$ sucrose, and $15 \%$ gelatin $/ 15.8 \%$ fructose matrices. 


\section{References}

1. te Booy MPWM, de Ruiter RA, de Meere ALJ. Evaluation of the Physical Stability of Freeze-Dried Sucrose-Containing Formulations by Differential Scanning Calorimetry. Pharm Research 1992;9:109-14.

2. Digenis GA, Gold TB, Shah VP. Cross-linking of gelatin capsules and its relevance to their in vitro-in vivo performance. J Pharm Sci 1994;83(7):915-921.

3. Bubnis WA, Ofner III CM. The determination of $\varepsilon$-amino groups in soluble and poorly soluble proteinaceous materials by a spectrophotometric method using trinitrobenzenesulfonic acid. Analyt Biochem 1992;207:129-133.

4. Martin A, Swarbrick J, Cammaruta A. In: Physical Pharmacy. Philadelphia:Lea \& Febiger, 1983. p. $566 \& 615$.

5. Kouassi K, Roos YH. Glass transition and water effects on sucrose inversion by invertase in a lactose-sucrose system. J Agric Food Chem 2000;48:2461-5.

6. Hagiwara T, Hartel RW. Effect of sweetener and storage temperature on ice crystallization in ice cream. J Dairy Sci 1996;79:735-744.

7. Veis A. In: The Macromolecular Chemistry of Gelatin. New York:Academic Press, 1964. p 90-99. 


\section{Chapter IV}

\section{Process Design and Optimization}

Since gelatin materials are known to be very sensitive to conditions of manufacture, the process variables need to be considered when manufacturing the gelatin

matrices. These variables include gel casting method and curing conditions (temperature, humidity, time, and pressure effects).

\section{Methodology}

\section{Materials}

Gelatin disks were prepared using gelatin Type B 225 Bloom and D(-)-fructose purchased from Sigma (Sigma Chemical Co, St. Louis, MO). D(+)-sucrose was purchased from Fluka Chemical Co., Milwaukee, WI.

\section{Casting Method}

Previously in this laboratory, Tewari [1] developed a gelatin formaldehyde crosslinked film that slowed the release of clindamycin. To cast a film of approximately $1 \mathrm{~mm}$ in thickness, a gelatin solution was poured on a glass plate covered with parafilm and the film was formed by pulling a knife blade across the solution. Reproducibility of films by others, however, was problematic. In order to increase the reproducibility among batches, a new technique was developed in this work by syringing a constant volume of the gelatin dispersion into Teflon coated wells, forming a circular gelatin disk matrix.

Along with the improvement of the casting technique, several parameters within the technique were evaluated to determine their importance when casting the gelatin 
disks. The first parameter that was evaluated was the bore size of the needle. Two casting temperatures $\left(73\right.$ and $\left.80^{\circ} \mathrm{C}\right)$ were also evaluated to determine casting temperature effects.

\section{Curing Method}

Water is known to act as a plasticizer [2]. Plasticization, on a molecular level, leads to increased molecular space of free volume and decreased local viscosity [3, 4]. An increase in the intermolecular space due to excess water will cause an increase in the rate in which a drug is released from the gelatin matrix. The presence of water plays a major role in the performance of the gelatin matrices. Thus, there is a need for a drying and curing step in the processing of these materials. During this phase, various drying processes were assessed for their ability to form a strong and dry matrix.

Refrigeration is an air-drying process that utilizes the constant temperature and humidity aspects of a closed system. The refrigeration process requires long curing times to produce samples that have the strength and structure of the material necessary for a sustained release delivery system.

Vacuum Drying is a drying process in which a vacuum is applied to an isolated sample in a sealed drying apparatus. Vacuum drying is a constant temperature, humidity, and pressure process and requires intermediate drying times. The vacuum drying process is advantageous over refrigeration because it can reduce the curing time. It is still a limited process because it dries the disk at a quick rate, 'freezing' the disks in such a manner that creates large interconnecting pores. 
Lyophilization is a drying process used to optimize the extent of cross-linking between gelatin and sugars in order to achieve greater physical entanglements within the matrix. Lyophilization is a low pressure, low temperature process. During this process, called sublimation, the product remains frozen while the water goes directly from the solid phase to the vapor phase without passing through the liquid phase. An ice crystal is composed of pure water that is rather rigidly confined in a crystal lattice [5]. This rigid confirmation of the ice crystals leads to a more ordered structuring of the polymer molecules during the freezing phase of drying. The molecules have natural vibrations so that extra thermal energy increases the probability of breaking free. When the water molecule breaks free, it diffuses through the already dried surface of the solid and sublimes. As the water molecules diffuse and sublime, the thickness of the dry outer surface of the specimen increases, and thus more energy is required to transport the molecules through the dry shell. The actual force driving water vapor from the drying boundary, through the dry shell and to the specimen surface, is a concentration gradient, and not the vacuum pulling on the sample [5]

The simplest form of the lyophilizer consists of a vacuum chamber into which wet sample material can be placed, together with a means of removing water vapor so as to freeze the sample by evaporative cooling and freezing and then maintain the water-vapor pressure below the triple-point pressure. The temperature of the sample would then continue to fall below the freezing point and sublimation would slow down until the rate of heat gain in the sample by conduction, convection, and radiation was equal to the rate of heat loss as the more energetic molecules sublimed away are removed [5]. 


\section{Refrigeration}

Gelatin disks were placed in the refrigerator (Westinghouse) for a minimum of 2 hours and up to 7 days. The refrigerator was maintained between 2 and $6^{\circ} \mathrm{C}$ and designated for this research. The refrigerator was only opened to remove samples and record the temperature twice daily.

\section{Vacuum Drying}

An Abderhalden drying apparatus (Aldrich, Milwaukee, WI) was used to isolate the sample while a vacuum was applied to dry the sample. Samples were removed from the drying apparatus at designated curing times. The samples were cut from the disk into $6 \mathrm{~mm} \times 6 \mathrm{~mm}$ squares. After the samples were cut from the disks, the original disks were allowed to further cure under refrigerated conditions. The samples were weighed, vacuum pressure dried for either 30 minutes or 1 hour, and re-weighed. Both weights were recorded.

\section{Lyophilization}

Four samples were cut from each formulation after a designated refrigeration time $(2,24$, or 48 hours). The weights of the samples were recorded and each sample was placed in a scintillation vial. A Virtis Freezemobile 12 (Virtis, Gardiner, NY) was used to lyophilize samples at $150 \mathrm{mTorr}$ and $-60^{\circ} \mathrm{C}$ for 17,24 , or 36 hours.

\section{Experimental Design for Manufacturing Optimization Studies}

Gelatin matrices were cured over various time periods that incorporated one or two drying/curing methods. The different curing conditions were then compared to each other in order to rank the curing conditions. These curing methods were developed 
throughout Phases I \& II as more structured and stable matrices were needed to improve the performance of the gelatin materials. Performance studies were conducted in order to determine the optimal curing conditions. Optimal curing conditions consist of one or more drying steps that remove water from the matrix while achieving the greatest amount of physical entanglements within the matrix. Time and efficiency of the operation were also taken into consideration.

\section{Results}

\section{Casting Method}

The incorporation of the new casting method demonstrated an increased reproducibility among the gelatin disks. As the method became more defined, the diameter and thickness of the gelatin matrices became constant. This was seen upon visual observation, however, reproducibility was ultimately seen in some of the performance studies (swelling/erosion and release) where the standard deviation was tighter than seen with Tewari's [1] method. As the gelatin solution is heating and cooling, small visible tendrils form within the solution.

The force in which the solution is pushed through the needle may change or break the tendrils. According to Poiseuille's law for liquid flowing through a capillary, $\eta=$ $\pi r^{4} t \Delta \mathrm{P} / 81 \mathrm{~V}$ (where $\eta$ is the viscosity of the liquid, $r$ is the radius of the inside of the capillary, $\mathrm{t}$ is the time of flow, $\Delta \mathrm{P}$ is the pressure head under which the liquid flows, 1 is the length of the capillary, and $\mathrm{V}$ is the volume of liquid flowing), a change in the radius of the capillary will inversely affect the pressure [6]. Thus, three bore sizes were compared when syringing the gelatin solution into the Teflon wells. The 12 gauge needle 
did not work well because the bore of the needle was too small. The pressure/resistance was too great to allow the gelatin to pass through the needle. Little to no differences were seen between the 16 and 20 gauge needles. Swelling and erosion studies did not indicate that there were any differences between the two needle sizes. The bore size that was used throughout the research was a 20 gauge needle.

The casting temperature of the gelatin solution is extremely important. When water is used as the solvent, there is always concern for hydrolytic destruction of the gelatin. In order to limit the destruction of the gelatin molecule it is necessary to maintain temperatures below $80^{\circ} \mathrm{C}$ [7]. This temperature can only be exceeded for short periods of time. However, in order for the gelatin to go into solution (dispersion), undergo strand disentanglement, and react with cross-linking agents, the temperature must be maintained above $35^{\circ} \mathrm{C}$. As the gelatin dispersion temperature increases, the fluidity of the dispersion increases leading to a decrease in the viscosity. The dependence of viscosity on temperature is expressed by an equation analogous to the Arrhenius equation of chemical kinetics: $\eta=\mathrm{Ae}^{\mathrm{Ev} / \mathrm{RT}}$ where $\mathrm{A}$ is a constant depending on molecular weight and molar volume, $\mathrm{E}_{\mathrm{v}}$ is the activation energy required to initiate flow between molecules, and $\mathrm{R}$ is a gas constant [6]. Experimentally, at $80^{\circ} \mathrm{C}$, the gelatin solution had a low viscosity that was easy to cast, while the $73^{\circ} \mathrm{C}$ solution cooled quickly and became more viscous. This high viscosity made it more difficult to syringe the $73^{\circ} \mathrm{C}$ solution into the wells due to the relationship between viscosity and pressure. There were no swelling and erosion differences despite rheological differences. The casting temperature that was ultimately used was $80^{\circ} \mathrm{C}$. 


\section{Curing Method}

The initial phase of the study evaluated the performance of the gelatin and gelatin/sugar matrices over a seven day refrigeration period. During the curing process, the disks started out jelly-like, then became stiff and rubber-like as the disks solidified to a greater extent. Figures 15-17 show the swelling and erosion behavior of all three formulations, tested each day in a sequence of seven consecutive days of refrigeration. A desirable swelling and erosion profile includes a slow initial swelling followed by a plateau and erosion.

In the first two days of refrigeration, the matrices of all formulations did not perform well due to excess water being present in the matrix (limited drying). As seen in Figure 15, the non-cross-linked gelatin disks demonstrated a more desirable erosion profile after 3 and 4 days of refrigeration, though percent swelling was greater than with the sugar-based formulations. No significant swelling differences were seen when Days 5 through 7 were evaluated, though erosion time increased with increased drying time.

The gelatin/sugar matrices did not show the same progression in performance during the drying process that was seen with the non-cross-linked matrices. Figures $16 \&$ 17 show that the gelatin/sugar disks dry slowly over time with little improvement in the erosion profile in the first five days of drying. This may be due to the hygroscopic properties of the sugars resulting in the need for a longer curing time. Disks cured for 6-7 days show significant improvement in the swelling and erosion profiles. However, the long curing time makes the refrigeration process an undesirable curing method in the 


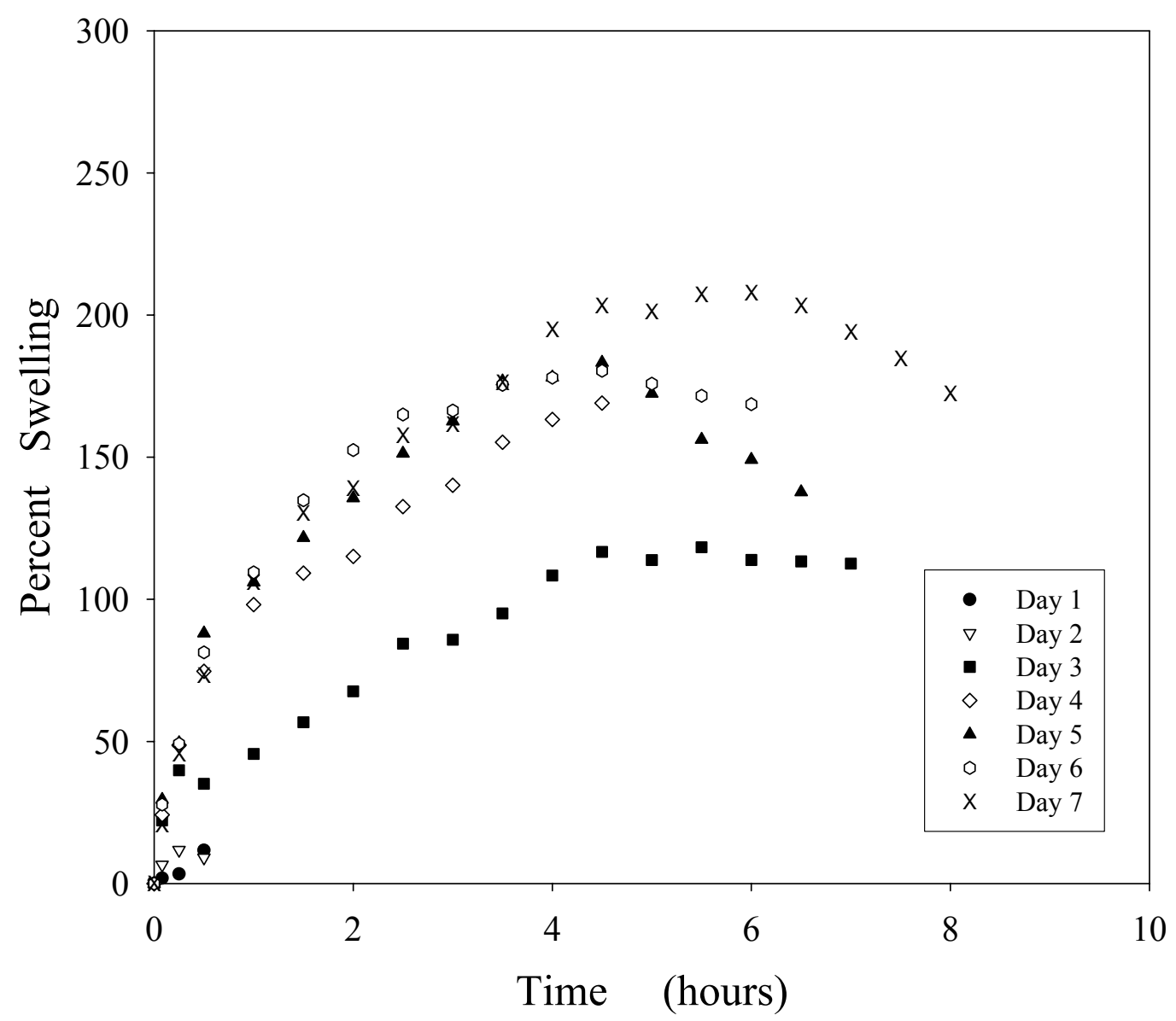

Figure 15. Swelling and erosion profile of gelatin matrices over a 7 day refrigeration period.

Matrices were evaluated under limited moisture and physiological temperatures. The results are plotted as an average of triplicate measurements. 


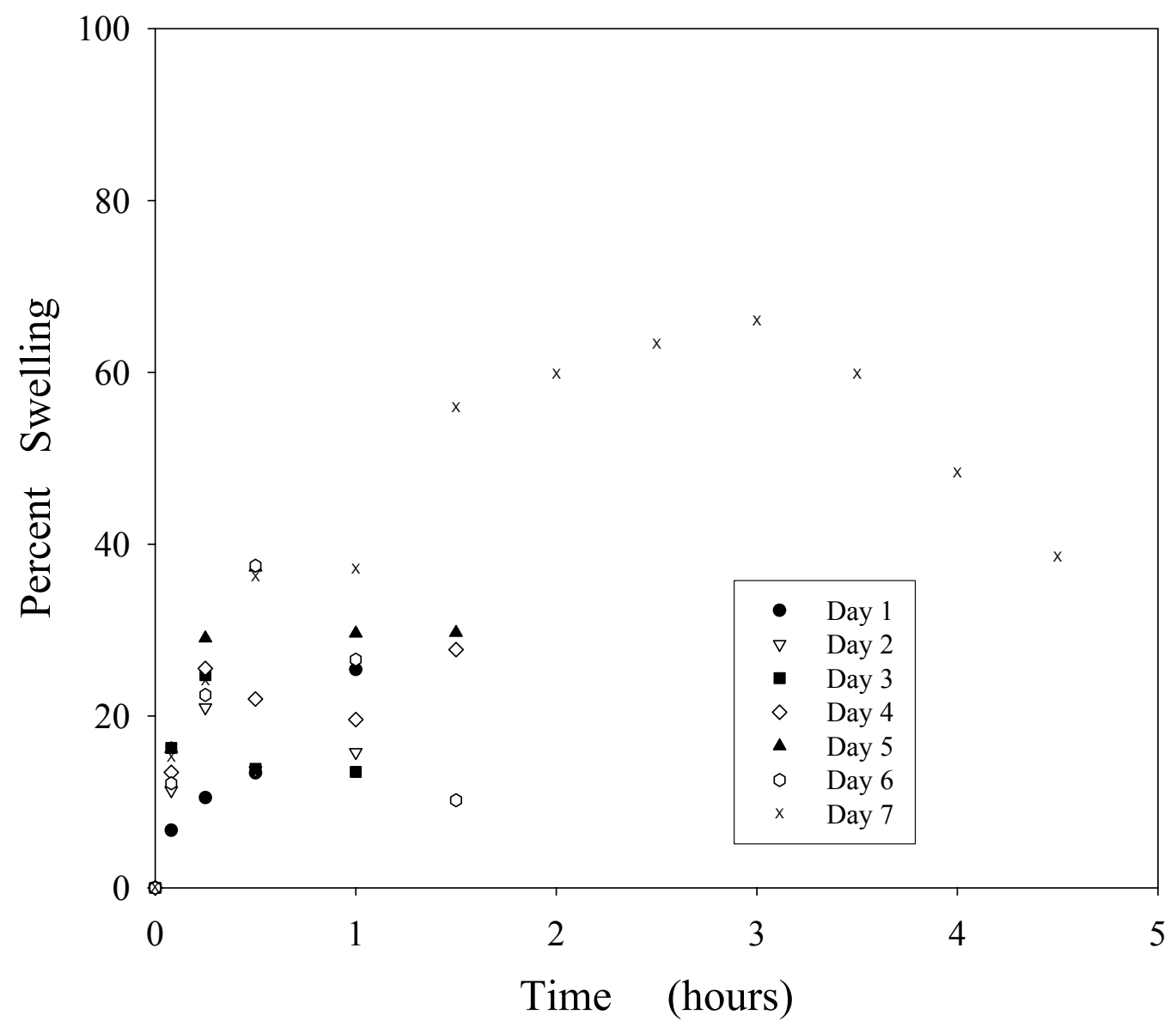

Figure 16. Swelling and erosion profile of gelatin/ $30 \%$ sucrose matrices over a 7 day refrigeration period.

Matrices were evaluated under limited moisture and physiological temperatures. The results are plotted as an average of triplicate measurements. 


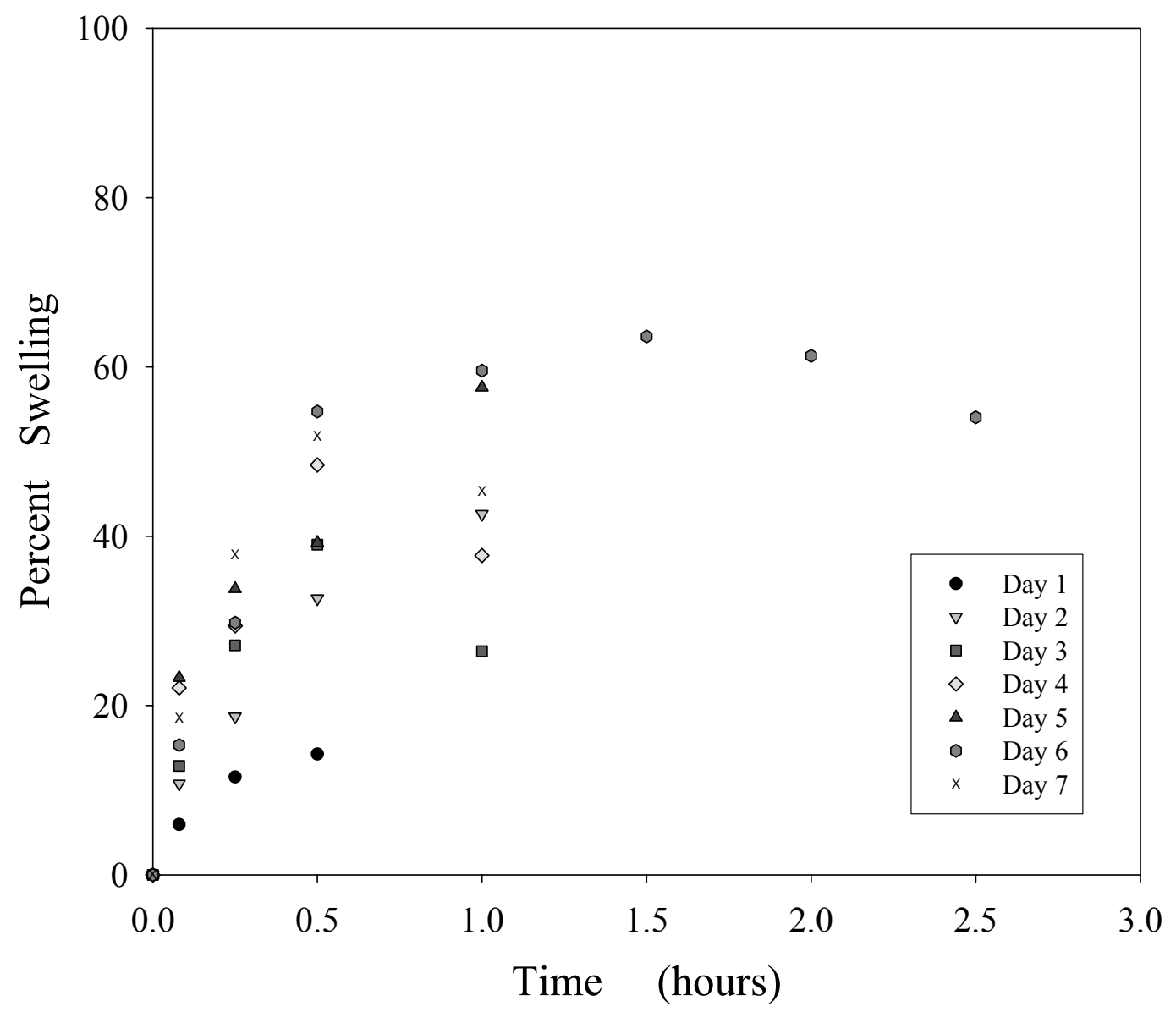

Figure 17. Swelling and erosion profile of gelatin/15.8\% fructose matrices over a 7 day refrigeration period.

Matrices were evaluated under limited moisture and physiological temperatures. The results are plotted as an average of triplicate measurements. 
industrial setting. As the disks cured, matrix performance improved due to increased order and stiffening of the strands (due to hydrogen bonding) within the matrix. In comparison with the non-cross-linked matrices, the sugar-based formulations (after 6-7 days of curing) demonstrated a lower percent swelling ( $\sim 60 \%)$ than did the non-crosslinked matrices (180-200\%) (Figure 18). However, the non-cross-linked matrix remained intact out to 8 hours, while the sugar-based formulations lasted 2.5-4.5 hours.

Thermal analysis was performed on these matrices. An important observation seen is the slight movement of peaks to the right (higher temperatures) after each 24 hour curing period (Figure 19-21). This is likely due to the formation of a drier and more rigid polymer network, making the matrices thermally more stable. A stable matrix will have an extremely high melting temperature when compared to a less structured matrix. These results indicate that the energy necessary to melt the matrix increases with an increase in drying time, and support the fact that chain rigidity increases due to loss of water.

The vacuum drying process allowed the matrix to be dehydrated to a greater extent than refrigeration alone. A loss of water during vacuum drying had been seen after each 24 hour refrigeration process with the 24 hour curing time losing the most and day 4 losing the least percent of water, as depicted in Table 4 . Table 4 also represents inter-day studies demonstrating the reproducibility of the gelatin and gelatin/sugar matrices. This table shows the large amount of water driven out of the gelatin matrices compared to the gelatin/sugar matrices. With the cross-linking agents present, there is less free space for the water to move within the polymer structure due to the water being more fully 


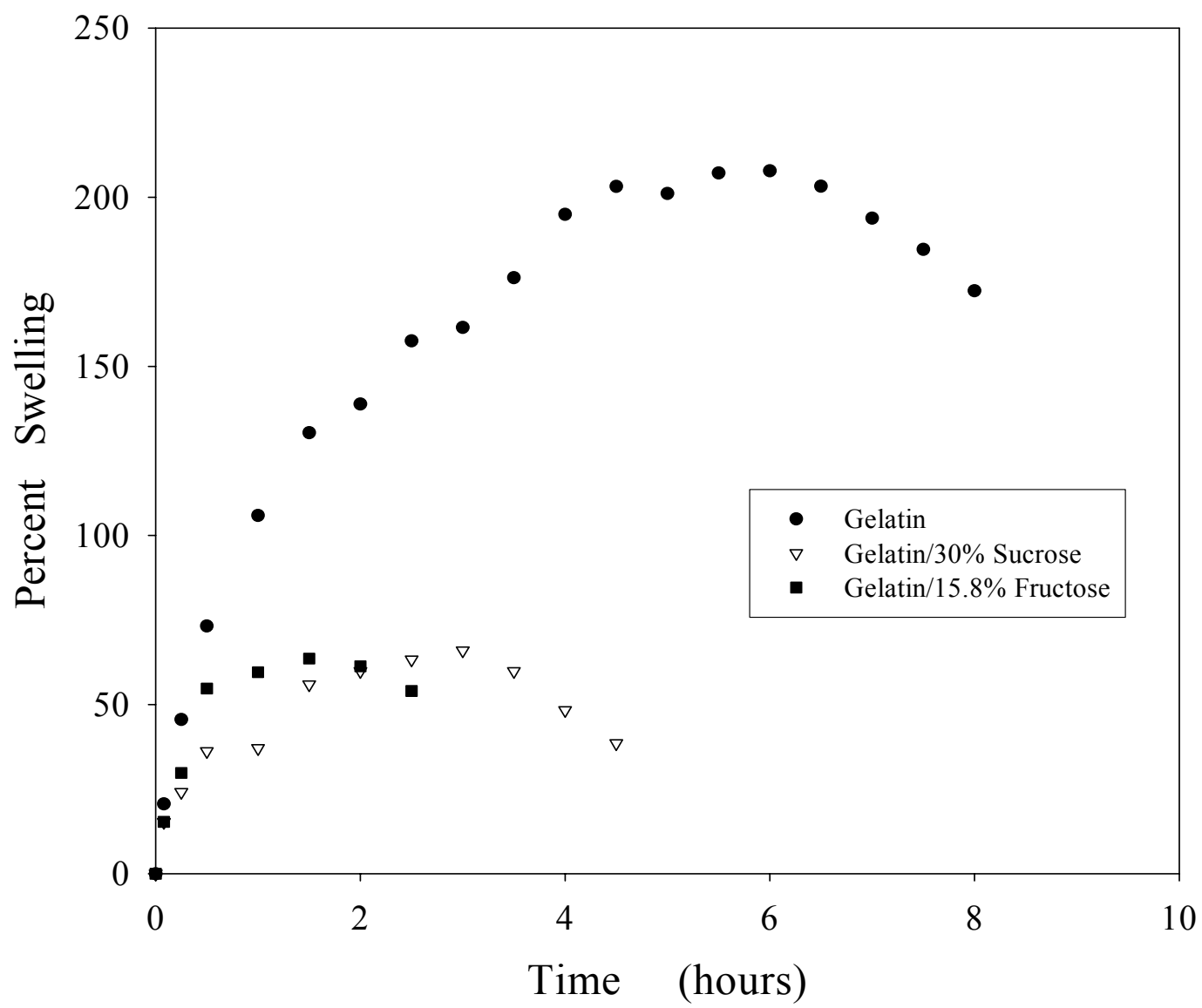

Figure 18. Swelling and erosion profile of gelatin, gelatin/30\% sucrose, and gelatin/15.8\% fructose matrices refrigerated for 7 days (non-cross-linked and sucrose) and 6 days (fructose).

Matrices were evaluated under limited moisture and physiological temperatures. The results are plotted as an average of triplicate measurements. 


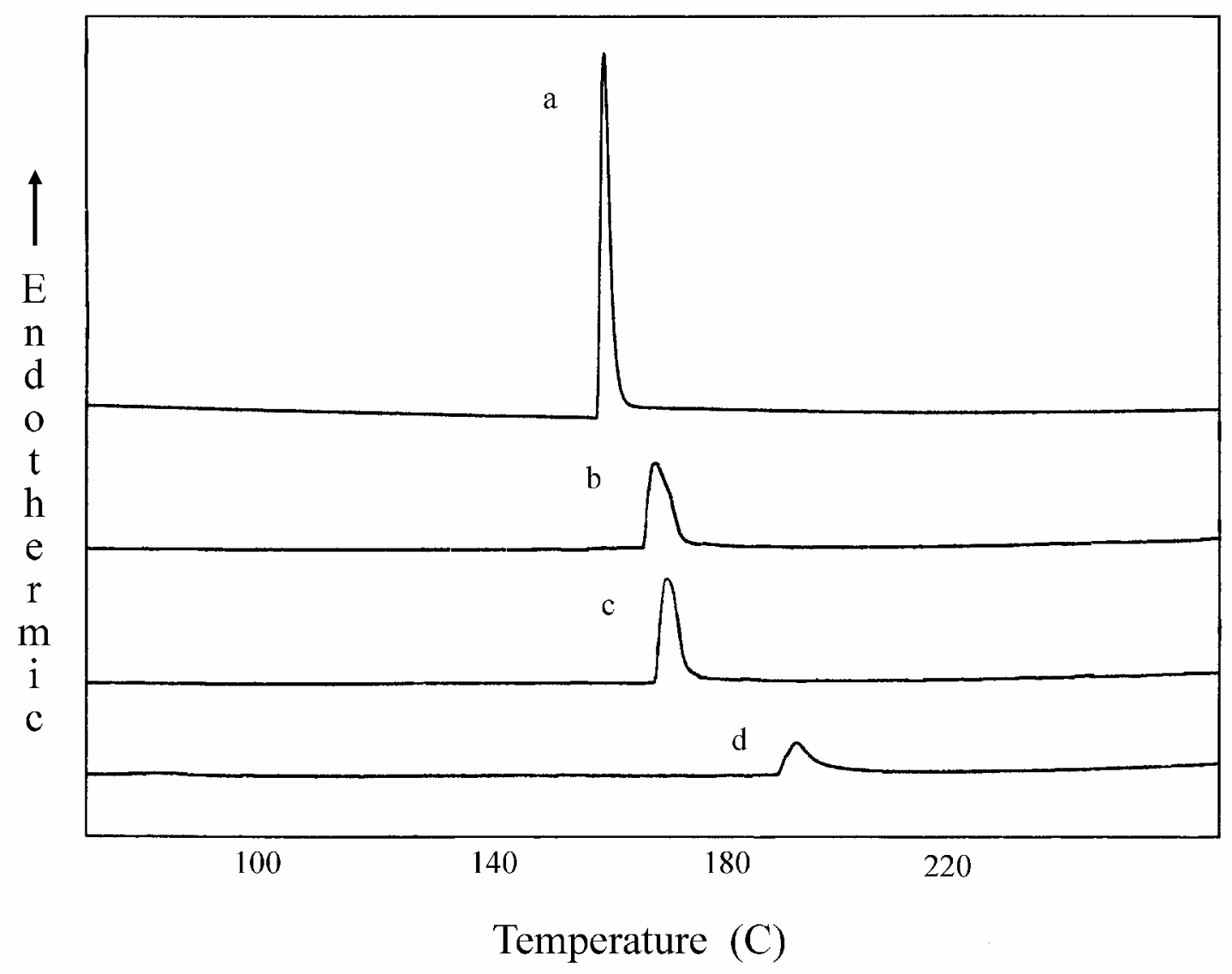

Figure 19. Thermal analysis scans of the gelatin matrix.

Lines a-d represent the first four days of the refrigeration curing process. 


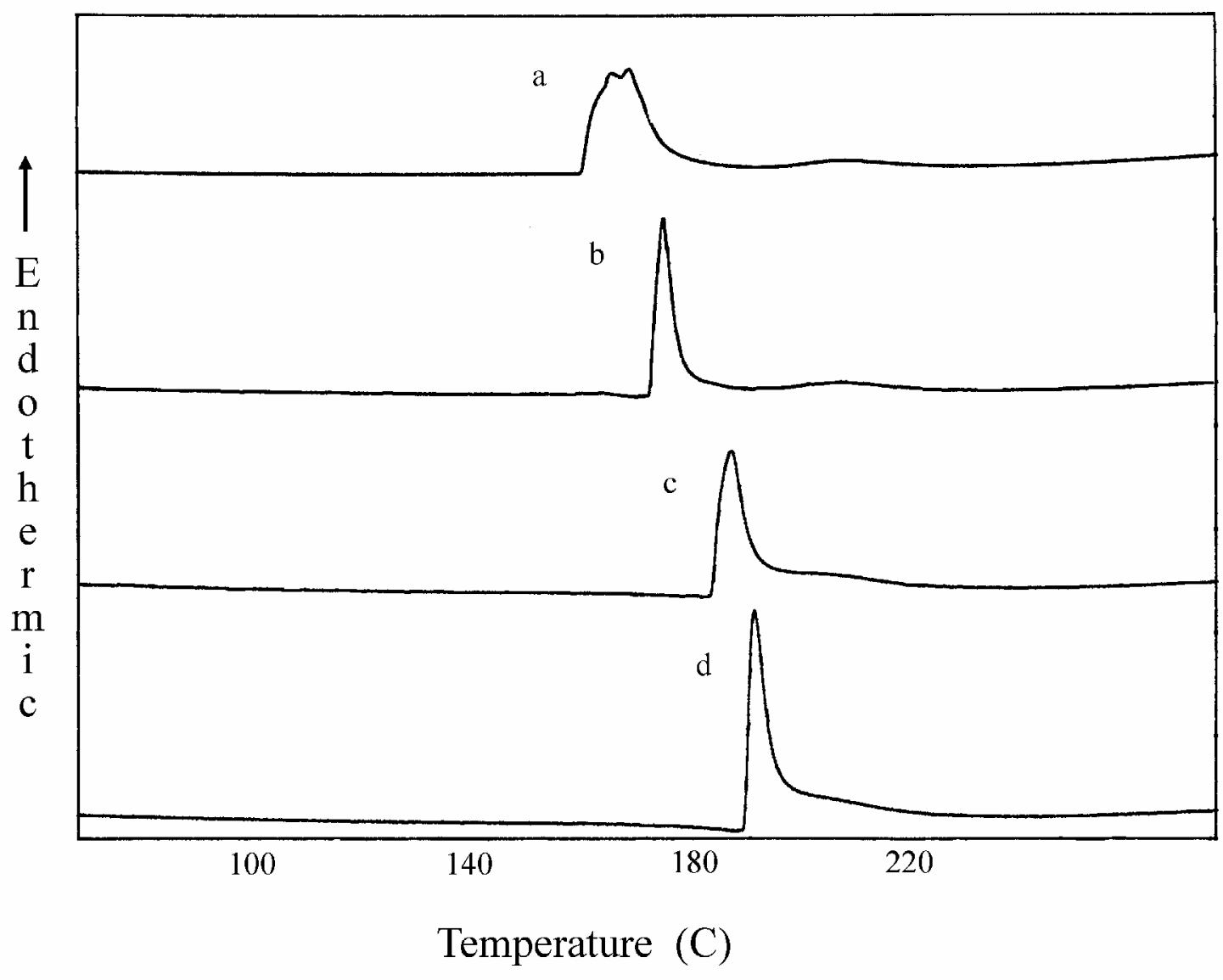

Figure 20. Thermal analysis scans of the gelatin/30\% sucrose matrix.

Lines a-d represent the first four days of the refrigeration curing process. 


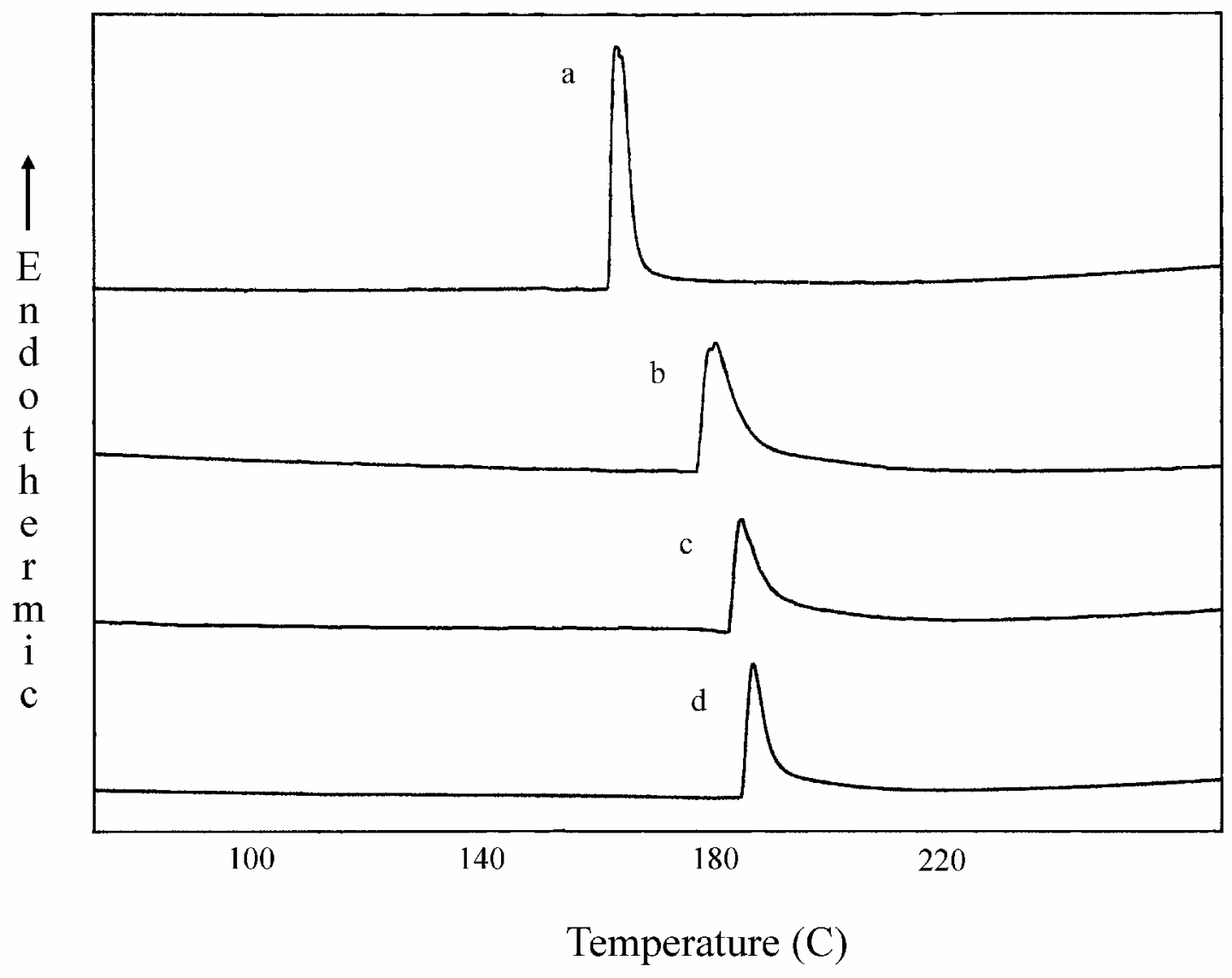

Figure 21. Thermal analysis scans of the gelatin $/ 15.8 \%$ fructose matrix.

Lines a-d represent the first four days of the refrigeration process. 
Table 4. Comparison of the percent water loss $( \pm S D)$ for the three formulations after the vacuum drying process was applied for 30 minutes over a 7 day refrigeration period.

Data represents a mean of 3 determinations.

\begin{tabular}{|c|c|c|}
\hline & $\begin{array}{c}\text { Gelatin/Sucrose } \\
(\% \pm \mathrm{SD})\end{array}$ & $\begin{array}{c}\text { Gelatin/no } \\
\text { cross-linking } \\
\text { agent }(\% \pm \mathrm{SD})\end{array}$ \\
\hline Day 1 & $37.7 \pm 1.7$ & $72.2 \pm 2.4$ \\
\hline Day 2 & $27.1 \pm 3.4$ & $67.7 \pm 2.5$ \\
\hline Day 3 & $12.7 \pm 1.6$ & $55.9 \pm 3.4$ \\
\hline Day 4 & $5.6 \pm 0.8$ & $7.2 \pm 1.8$ \\
\hline Day 5 & $3.3 \pm 0.7$ & $5.4 \pm 1.2$ \\
\hline Day 6 & $2.7 \pm 1.3$ & $4.9 \pm 0.1$ \\
\hline Day 7 & $2.7 \pm 0.3$ & $5.6 \pm 0.6$ \\
\hline
\end{tabular}

\begin{tabular}{|c|c|}
\hline $\begin{array}{c}\text { Gelatin/Fructose } \\
(\% \pm \mathrm{SD})\end{array}$ & $\begin{array}{c}\text { Gelatin/no } \\
\text { cross-linking } \\
\text { agent }(\% \pm \mathrm{SD})\end{array}$ \\
\hline $43.0 \pm 4.1$ & $73.9 \pm 2.0$ \\
\hline $19.5 \pm 8.4$ & $68.0 \pm 1.0$ \\
\hline $8.7 \pm 3.6$ & $59.7 \pm 4.8$ \\
\hline $3.2 \pm 1.5$ & $4.6 \pm 1.5$ \\
\hline $2.6 \pm 1.0$ & $4.1 \pm 1.2$ \\
\hline $1.4 \pm 0.8$ & $3.6 \pm 1.2$ \\
\hline $1.0 \pm 0.1$ & $3.5 \pm 0.8$ \\
\hline
\end{tabular}


embedded within the matrix. The data presented in Table 4 is in agreement with the results seen in the refrigeration data. A minimal and similar weight loss across all formulations after four days indicates that a four day curing period is adequate for processing the gelatin matrices.

Thermal analysis data shows the shift to higher temperatures after a brief 30 minute drying period. The non-cross-linked matrices show a larger shift $\left(\sim 25^{\circ} \mathrm{C}\right)$ in the melting temperature after vacuum drying (Figure 22); a shift is also seen with the gelatin/sugar matrices though smaller (Figures $23 \& 24$ ). This may be due to the hygroscopic properties of the sugars causing the matrices to retain more water. This retention of water affects the matrices' physical texture but not its strength and stability. Also of note is that, prior to and after drying, the gelatin/sugar matrices exhibited a higher $\mathrm{T}_{\mathrm{m}}$ than the non-cross-linked matrices. In two cases (gelatin/fructose and non-crosslinked), the peak after vacuum drying was a single, not multiple peak, indicative of a loss of amorphous characteristics to the matrix with a gain in more ordered structure after drying. The peaks for gelatin/sucrose matrices were single, both before and after drying. This confirms that the vacuum drying process forms a strong, stable matrix.

The combination of the two curing processes (refrigeration and vacuum drying) improves the performance of the gelatin and gelatin/sugar matrices, but still has its limitations. The vacuum drying process utilizes a high pressure vacuum to remove water from the matrix. This pressure forces the water out of the matrix at an extremely fast rate, likely creating large pores. The existence of these pores may affect both the 


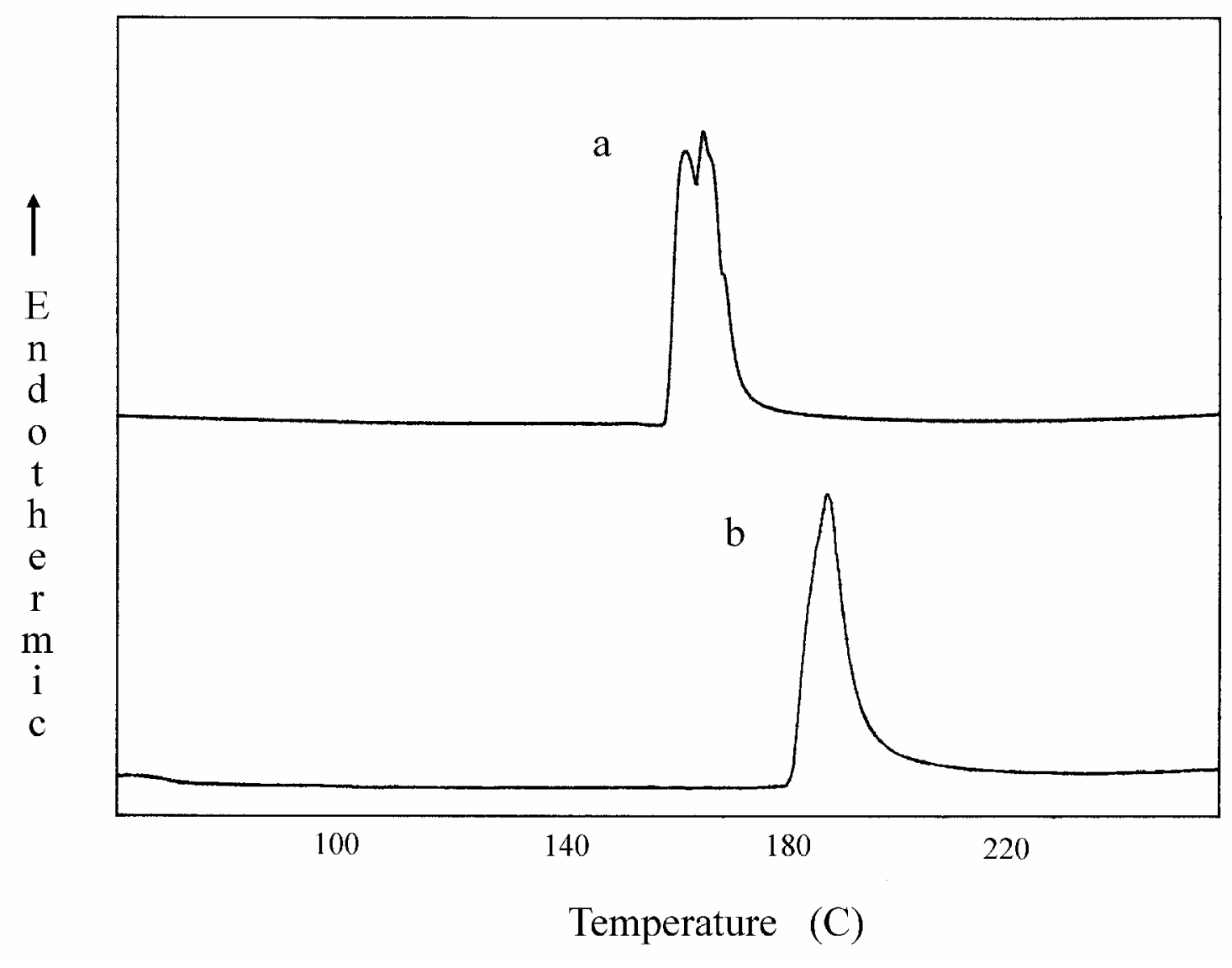

Figure 22. Thermal analysis scans of a gelatin matrix before (a) and after (b) a 30minute vacuum drying period.

Matrices were cured for 3 days at refrigerated temperatures. 


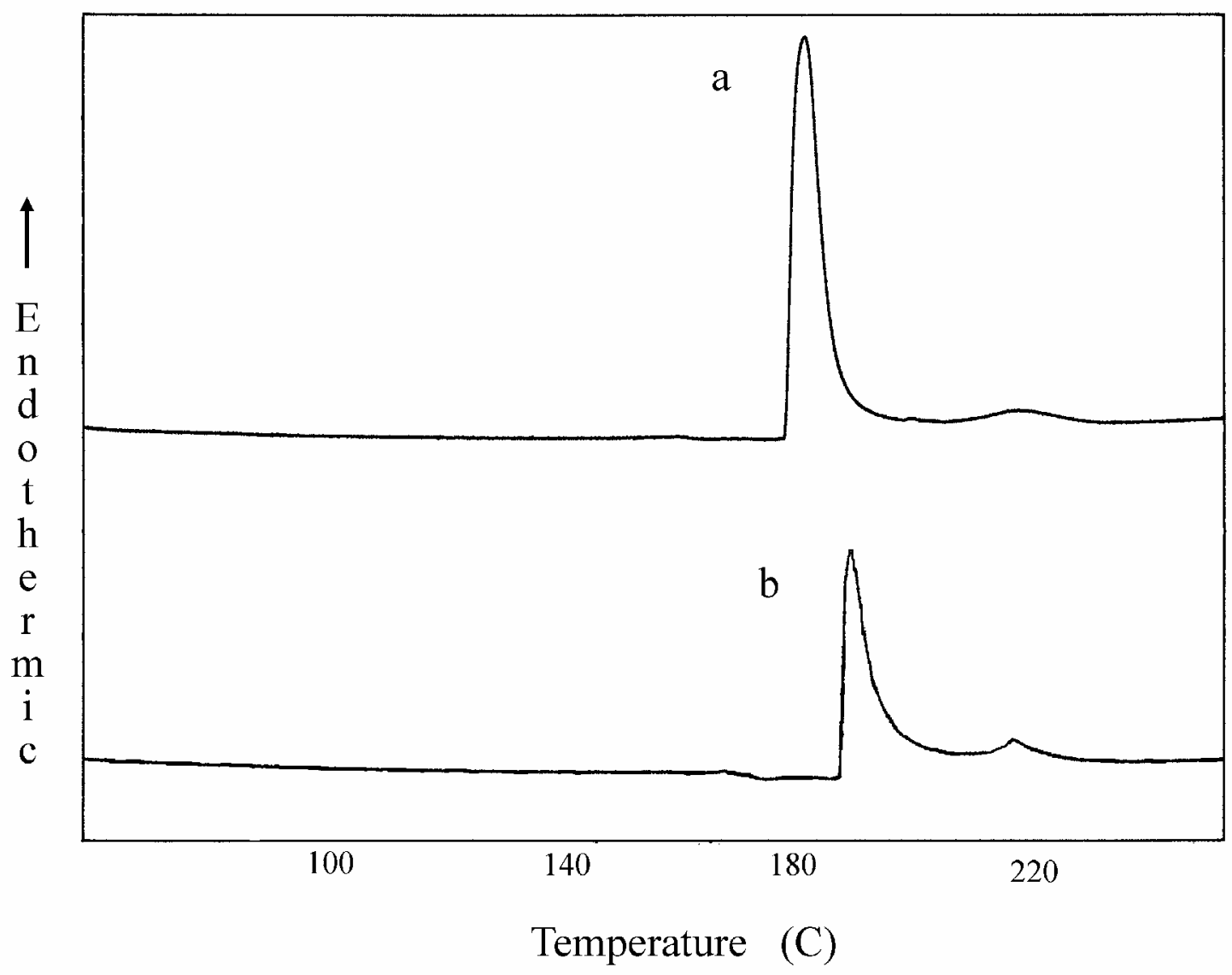

Figure 23. Thermal analysis scans of a gelatin/ $\mathbf{3 0 \%}$ sucrose matrix before (a) and after (b) a 30-minute vacuum drying period.

Matrices were cured for 3 days at refrigerated temperatures. 


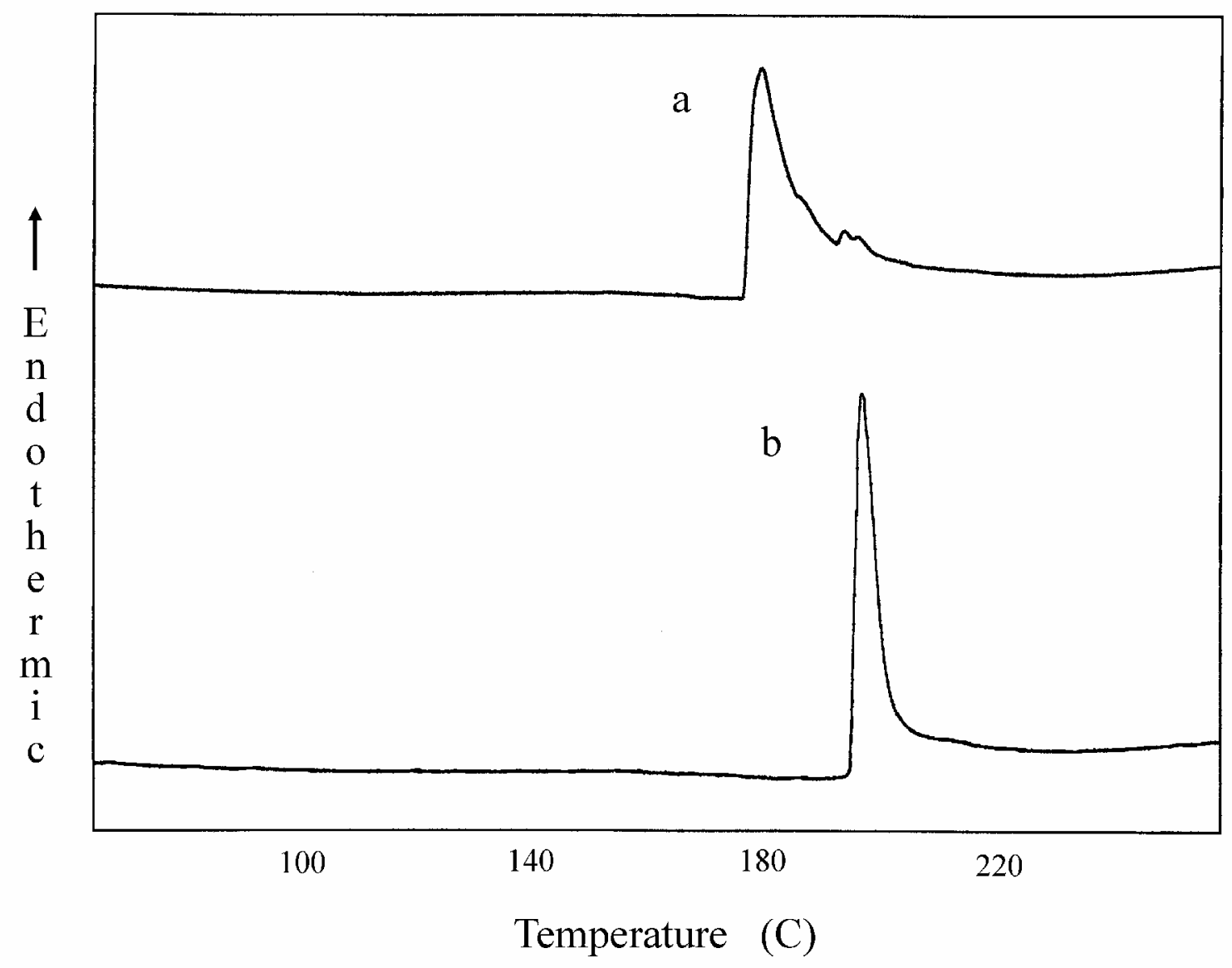

Figure 24. Thermal analysis of a gelatin/15.8\% fructose matrix before (a) and after (b) a 30-minute vacuum drying period.

Matrices were cured for 3 days at refrigerated temperatures. 
swelling and release performance of the matrix. This combination shows improvement, however, the combination still requires a lengthy curing period (4-5 days of refrigeration). In order to dry the matrices without creating large pores, the process of lyophilization was evaluated as a curing process.

In order to evaluate the effect that lyophilization has on the strength and performance of the matrices, several combinations of refrigeration and lyophilization times were performed. All lyophilized formulations demonstrated an improvement over refrigeration and vacuum drying. Upon lyophilization (after matrices were refrigerated for 2,24 , or 48 hours), the samples lost water, became smaller in size (1/3 of original size), and retained $40-70 \%$ of original weight. This is due to the relatively low amount of gelatin and cross-linking agents that were present in the matrix at the time the sample was cut from the disk. As the disks cure and lose water, they undergo syneresis, become smaller in size, and more highly concentrated with gelatin and/or sugar. A $10 \mathrm{~cm}$ square at 2 hours of curing has a lower concentration of gelatin than the same size sample after 24 hours of curing.

Prior to 2 hours of refrigeration, gelation has not fully occurred and the matrices are not easily handled. This makes the preparation for lyophilization prior to 2 hours not feasible. After 2 hours of refrigeration, the disks were congealed but still retained a large percentage of water. Due to the excess amount of water and low gelatin content, the disks were unable to form the necessary network within the matrix to create a strong, stable matrix. During the lyophilization process, water crystals are formed on freezing 
(immediately providing a more structured character to the matrix), removed from the outside in, and leave behind a more ordered and concentrated structure upon drying (sublimation). The sublimation process leaves behind crystallite zones and fewer amorphous regions.

These samples displayed a greater swelling profile and exhibited a faster erosion time than the other matrices (Figures 25-27). The extensive swelling profile may be due to the excess water present in the matrix upon initiation of the lyophilization process when the matrices are cured for 2 hours. As the lyophilization process forces water out of the matrix, the excess water may create large pores within the matrix. When the matrices are exposed to water, these matrices may be able to imbibe more water than matrices with small pores and swell to a greater extent. Also, the samples are relatively drier at the start of these studies than matrices processed by the other two methods. The samples refrigerated at 24 and 48 hours show a slight decrease in the overall swelling profile. This is due to the slow loss of water during the refrigeration phase which allows the gelatin strands more opportunity to arrange themselves into a structure which offers greater resistance to swelling. The addition of lyophilization further cures the matrix, decreasing the distance between strands and increasing the opportunities for physical entanglements, hydrogen bonds, and ionic interactions. In lyophilization, the gelatin has a tighter packing arrangement [8]. Since there is less water present in the matrix, the pore sizes formed during the lyophilization process are not as large as with the shorter refrigeration times. 


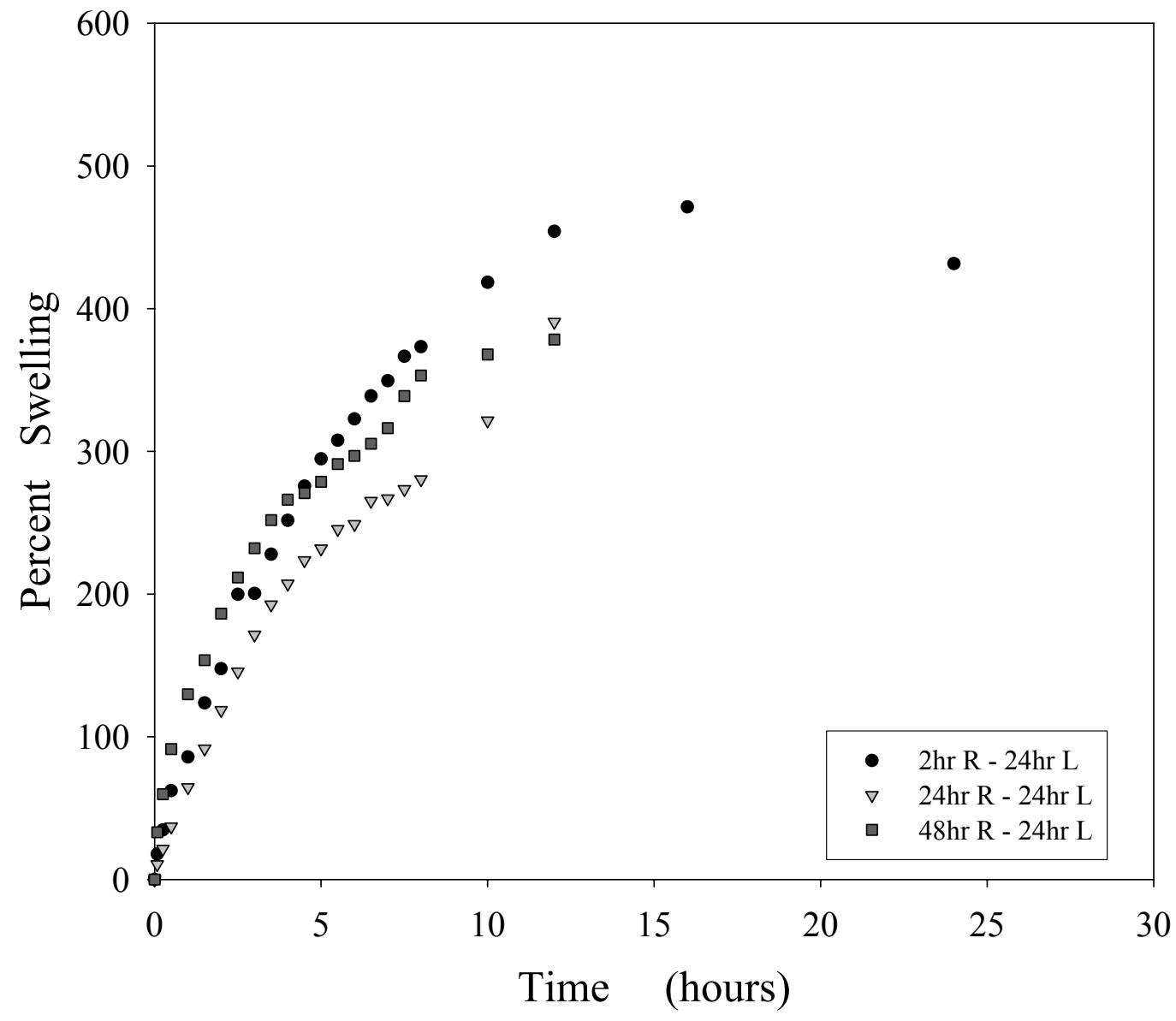

Figure 25. Swelling and erosion profile of gelatin matrices refrigerated, $\mathbf{R}$, for 2, 24, or 48 hours followed by 24 hours of lyophilization, $L$.

Matrices were evaluated under limited moisture and physiological temperatures.

The results are plotted as an average of triplicate measurements. 


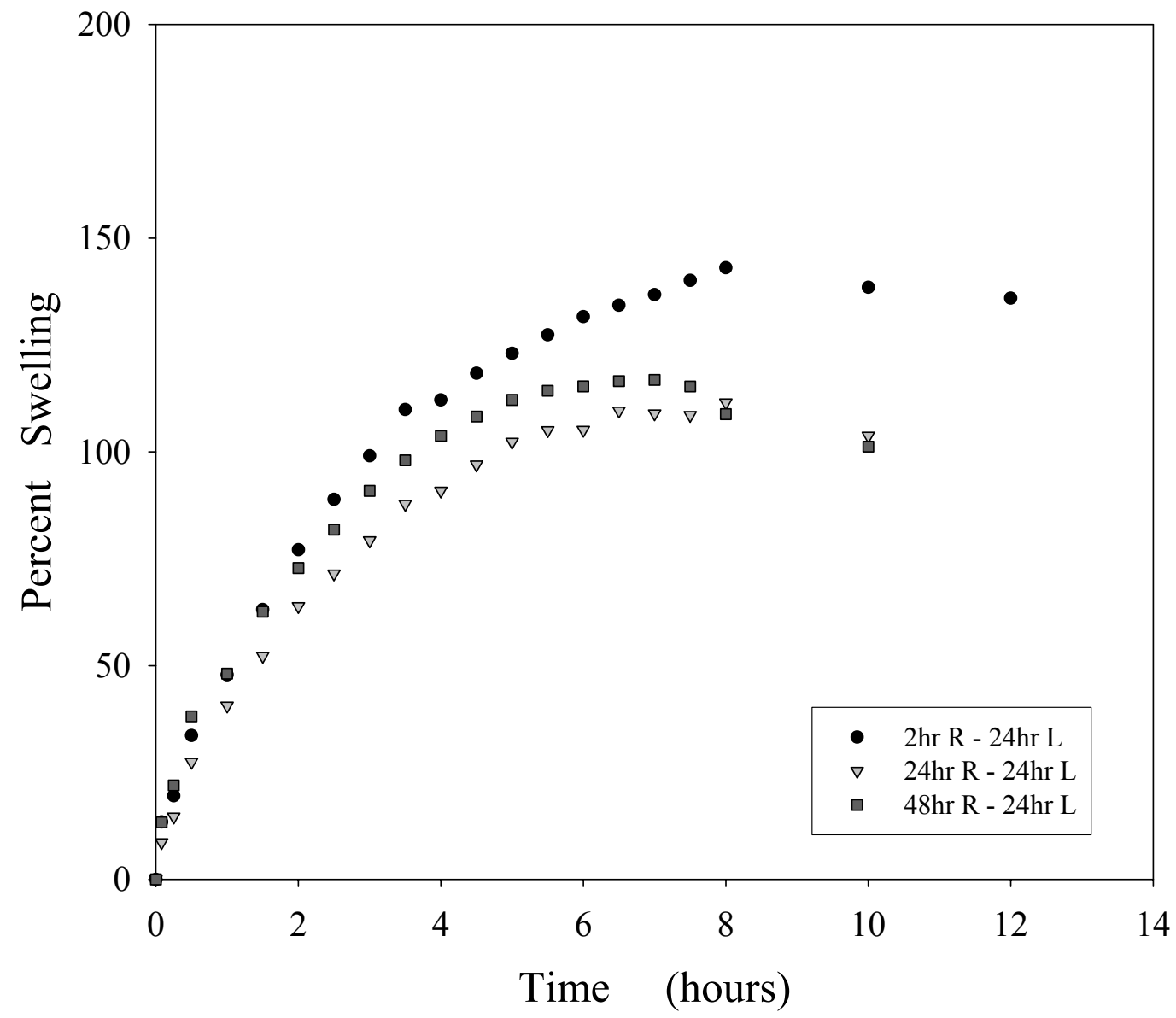

Figure 26. Swelling and erosion profile of gelatin/30\% sucrose matrices refrigerated, R, for 2, 24, or 48 hours followed by 24 hours of lyophilization, L.

Matrices were evaluated under limited moisture and physiological temperatures.

The results are plotted as an average of triplicate measurements. 


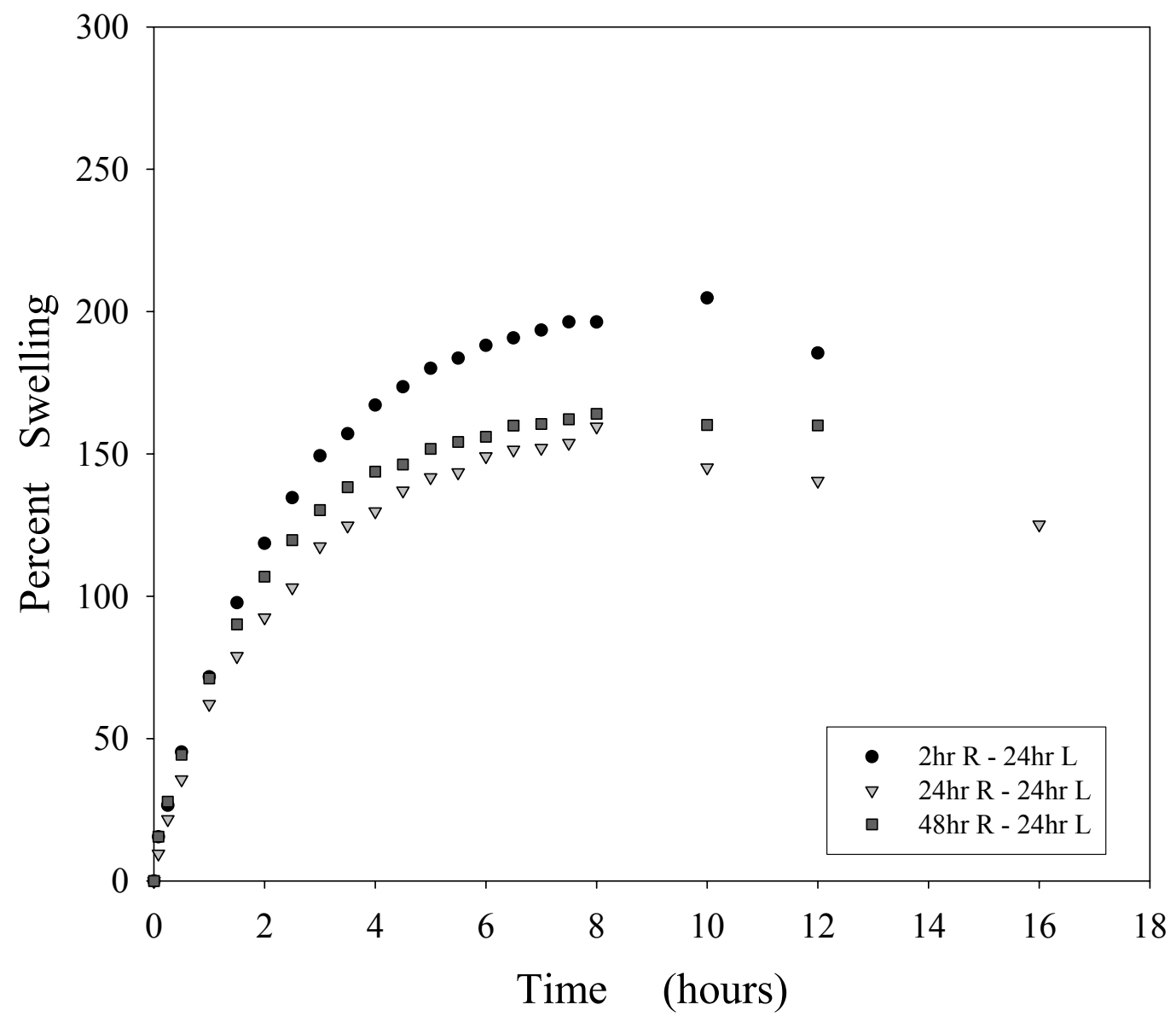

Figure 27. Swelling and erosion profile of gelatin/15.8\% fructose matrices refrigerated, R, for 2, 24, or 48 hours followed by 24 hours of lyophilization, L.

Matrices were evaluated under limited moisture and physiological temperatures. The results are plotted as an average of triplicate measurements. 
Two lyophilization times were also evaluated, 24 and 36 hours, with the hypothesis that a longer lyophilization time may be needed in order to reach the most ordered matrix structure. However, no significant differences were seen when the disks were lyophilized for 24 versus 36 hours (Figures 28-30). Both the percent swelling and time to erosion remained approximately the same. This indicates that a majority of the water is likely removed in the first 24 hours of the lyophilization process with little to no water being removed over the next 12 hours (Table 5), and that the matrix structural effects of drying occur within the first 24 hours of lyophilization. The matrices cured for 24 hours in the refrigerator followed by 36 hours of lyophilization demonstrated a more rapid erosion than the matrices refrigerated and lyophilized for 24 hours.

Finally, a comparison of a short refrigeration-short lyophilization, a medium refrigeration-medium lyophilization, and a long refrigeration-long lyophilization processed matrices was performed and the results are shown in Figures 31-33. All three formulations exhibited similar results. The $2 \mathrm{hr}$ refrigeration- $17 \mathrm{hr}$ lyophilization matrices had a low percent swelling and faster erosion time than the other two conditions. The low percent swelling is likely due to the presence of water within the matrix. The short curing times likely do not remove all of the water from the matrix, leaving small spaces within the matrix for the solvent to diffuse and act as a plasticizer (allowing for more ready movement of the gelatin strands). The quick erosion occurs because the gelatin strands remain mobile leaving the matrices susceptible to erosion throughout the swelling process. The other two curing combinations ( $24 \mathrm{hr}$ refrigeration- $24 \mathrm{hr}$ lyophilization and $48 \mathrm{hr}$ refrigeration-36 hr lyophilization) demonstrate similar profiles 


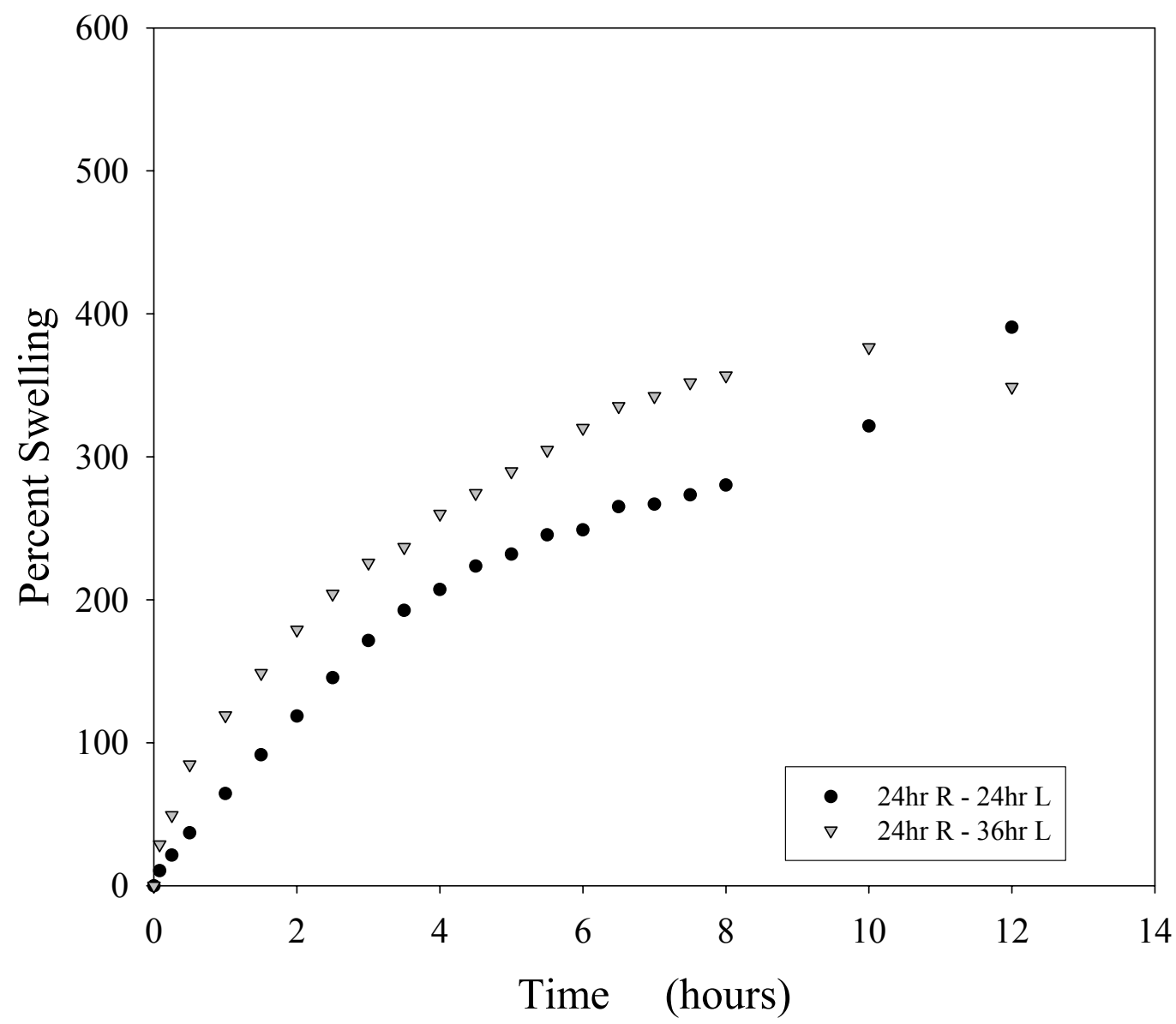

Figure 28. Swelling and erosion profile of gelatin matrices refrigerated, $\mathbf{R}$, for 24 hours and lyophilized, L, for either 24 or 36 hours.

Matrices were evaluated under limited moisture and physiological temperatures.

The results are plotted as an average of triplicate measurements. 


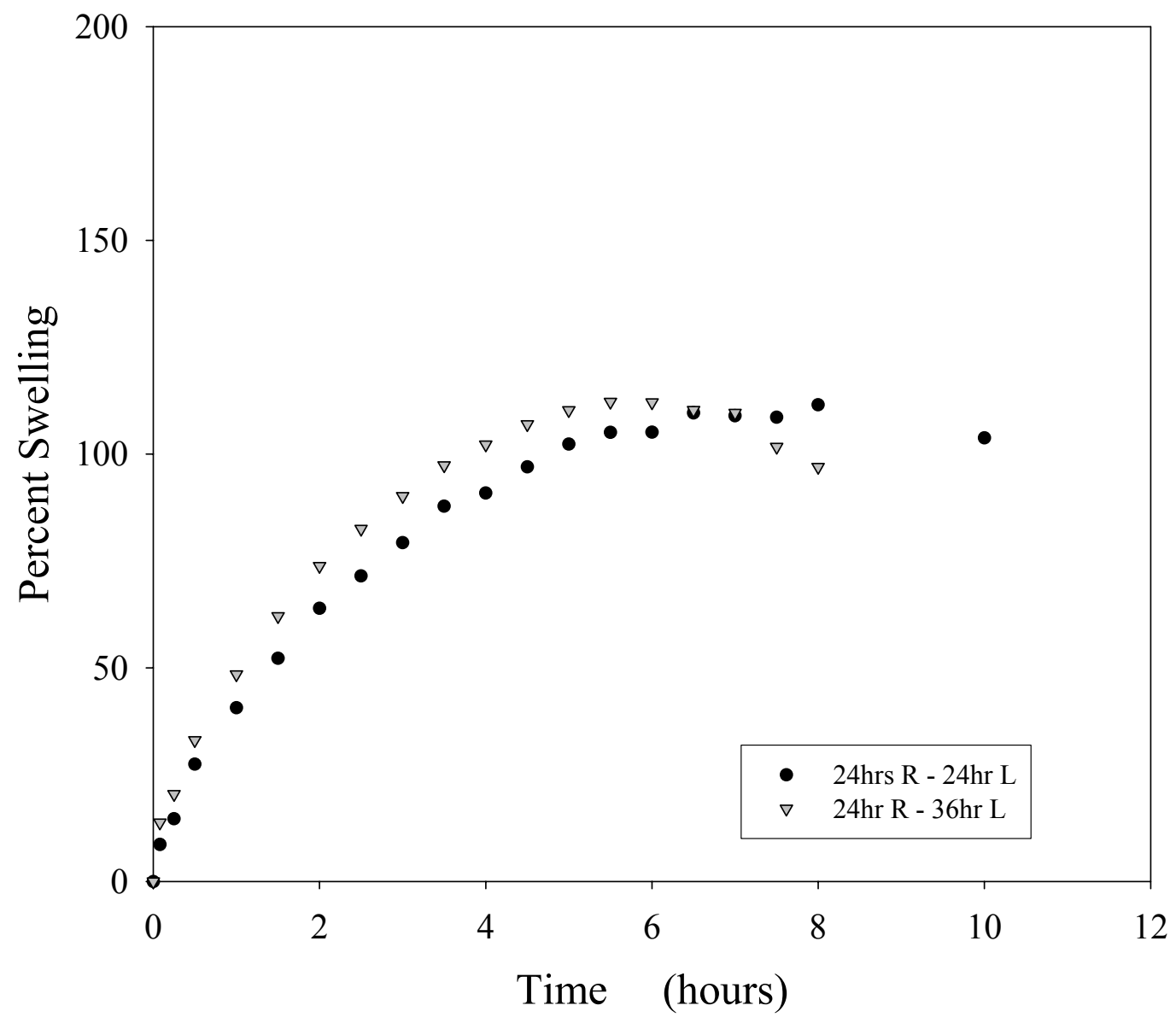

Figure 29. Swelling and erosion profile of gelatin/30\% sucrose matrices refrigerated for 24 hours and lyophilized for either 24 or 36 hours.

Matrices were evaluated under limited moisture and physiological temperatures.

The results are plotted as an average of triplicate measurements. 


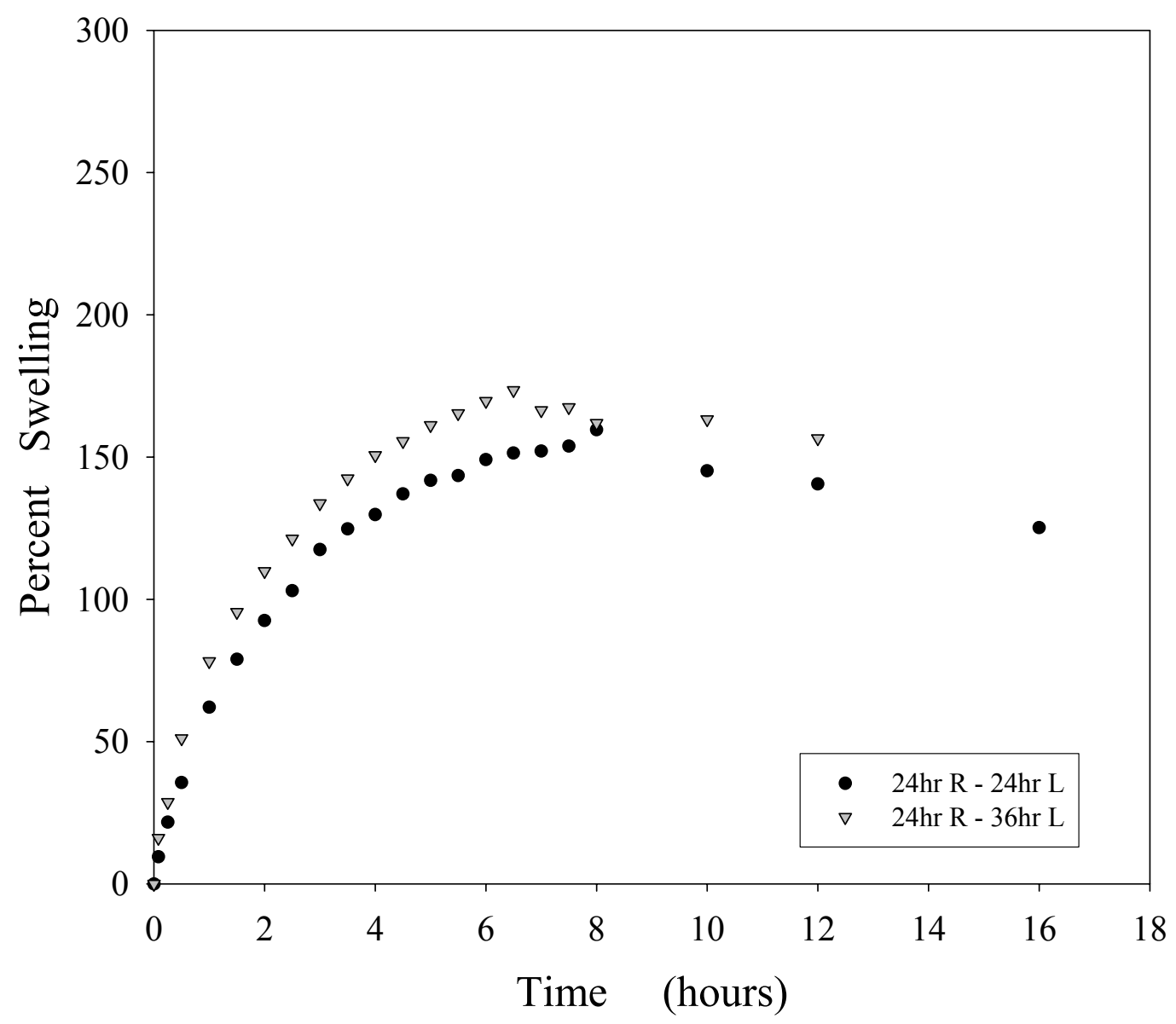

Figure 30. Swelling and erosion profile of gelatin/15.8\% fructose matrices refrigerated for 24 hours and lyophilized for either 24 or 36 hours.

Matrices were evaluated under limited moisture and physiological temperatures. The results are plotted as an average of triplicate measurements. 
Table 5. Comparison of water lost after samples were refrigerated for 24 hours followed by either 24 or 36 hours of lyophilization.

Data represents a mean of 12 determinations.

\begin{tabular}{|c|c|c|c|}
\hline $\begin{array}{c}\text { Sample } \\
\text { Set }\end{array}$ & $\begin{array}{c}\text { Pre-lyophilized } \\
\text { Weight } \\
(\mathrm{mg})\end{array}$ & $\begin{array}{c}\text { 24 hours } \\
\text { Lyophilization } \\
(\mathrm{mg})\end{array}$ & $\begin{array}{c}\text { 36 hours } \\
\text { Lyophilization } \\
(\mathrm{mg})\end{array}$ \\
\hline Gelatin & $80.03 \pm 3.2$ & $22.24 \pm 4.2$ & $20.67 \pm 4.9$ \\
\hline $\begin{array}{c}\text { Gelatin/ } \\
30 \% \text { Sucrose }\end{array}$ & $150.70 \pm 2.6$ & $83.00 \pm 3.9$ & $80.23 \pm 6.4$ \\
\hline $\begin{array}{c}\text { Gelatin/ } \\
15.8 \% \text { Fructose }\end{array}$ & $145.40 \pm 3.8$ & $59.78 \pm 5.0$ & $58.23 \pm 6.2$ \\
\hline
\end{tabular}




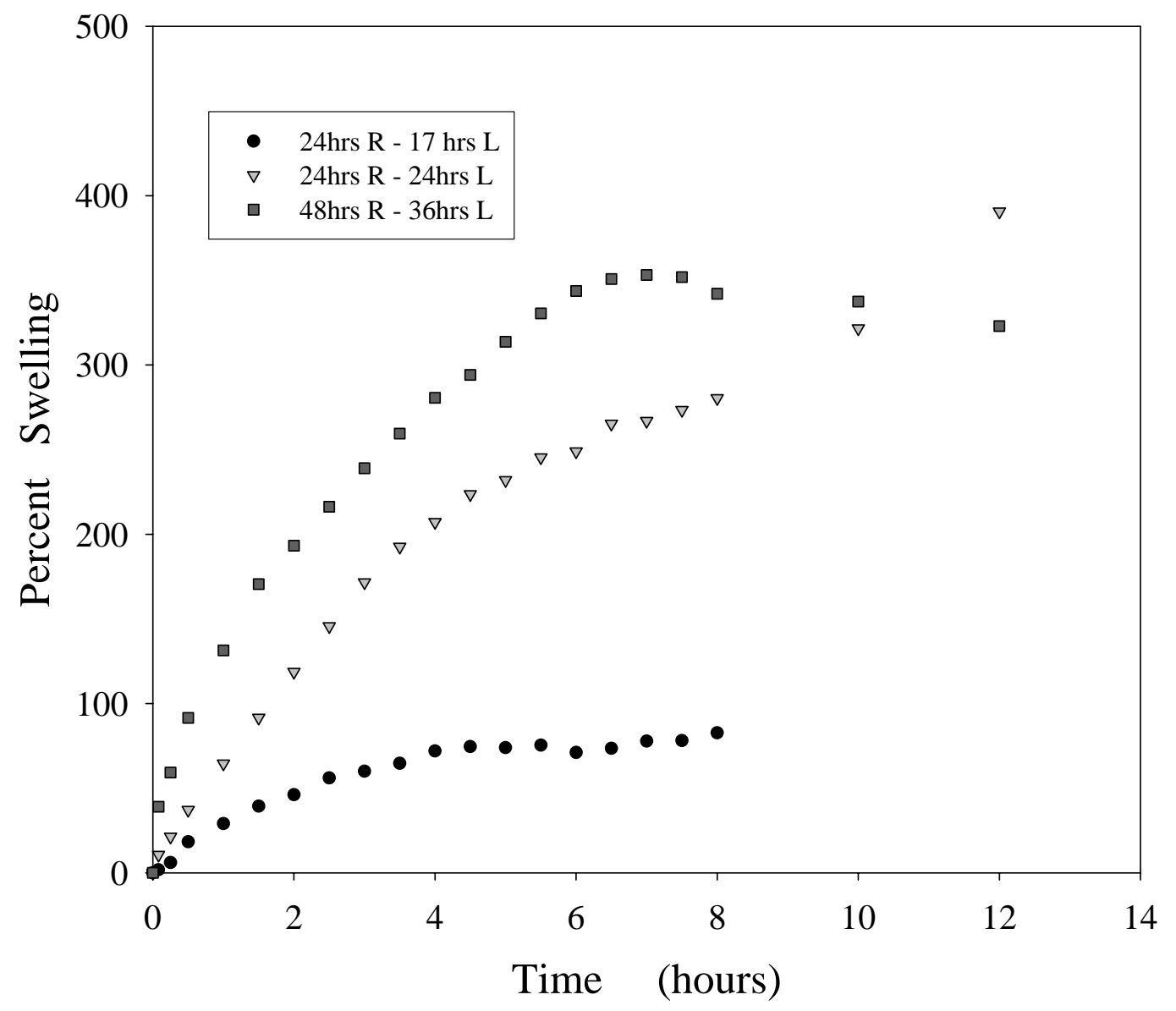

Figure 31. Swelling and erosion profiles of gelatin matrices refrigerated and lyophilized for short, medium, and long curing times.

Matrices were evaluated under limited moisture and physiological temperatures. The results are plotted as an average of triplicate measurements. 


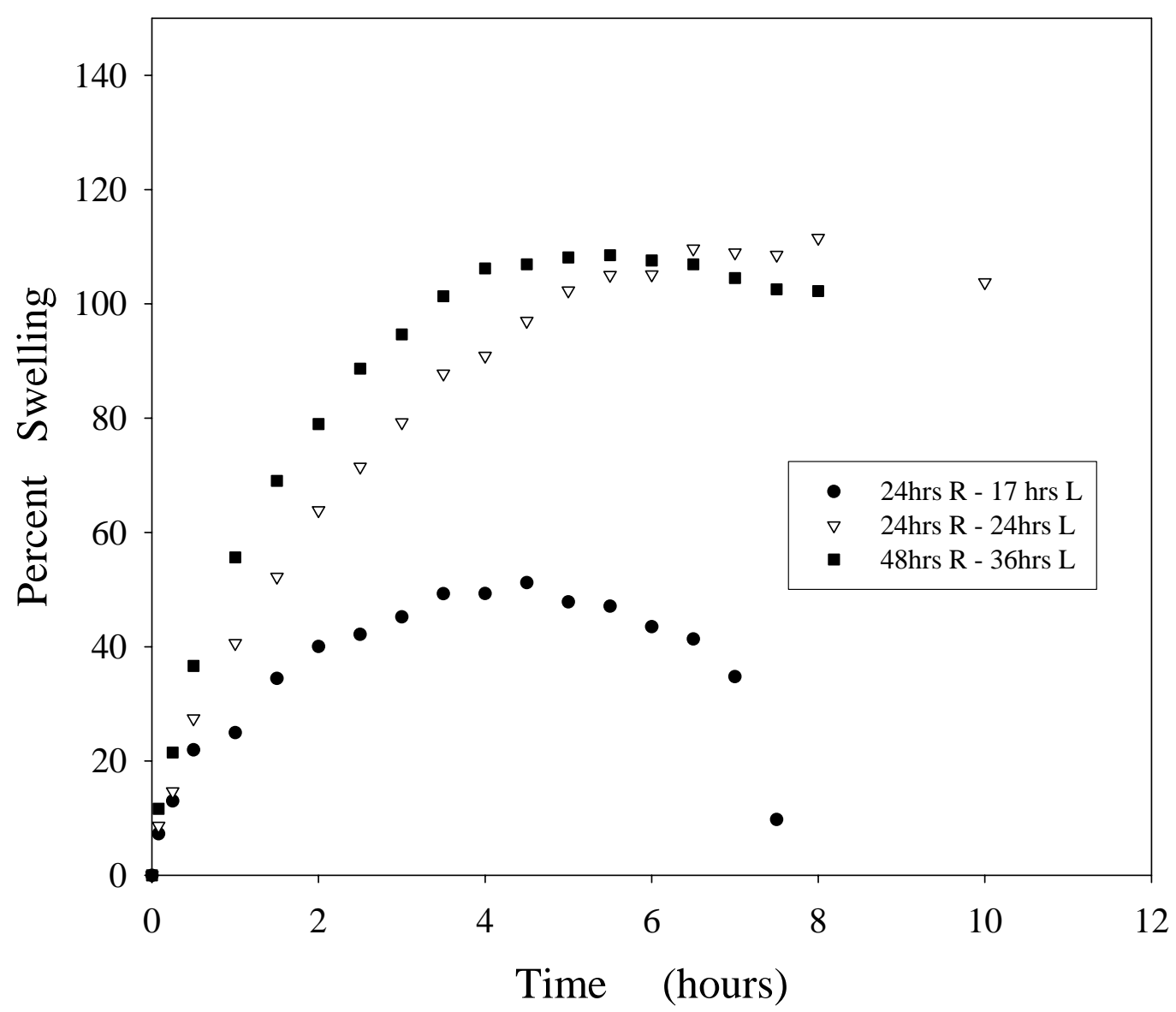

Figure 32. Swelling and erosion profiles of gelatin/30\% sucrose matrices refrigerated and lyophilized for short, medium, and long curing times.

Matrices were evaluated under limited moisture and physiological temperatures. The results are plotted as an average of triplicate measurements. 


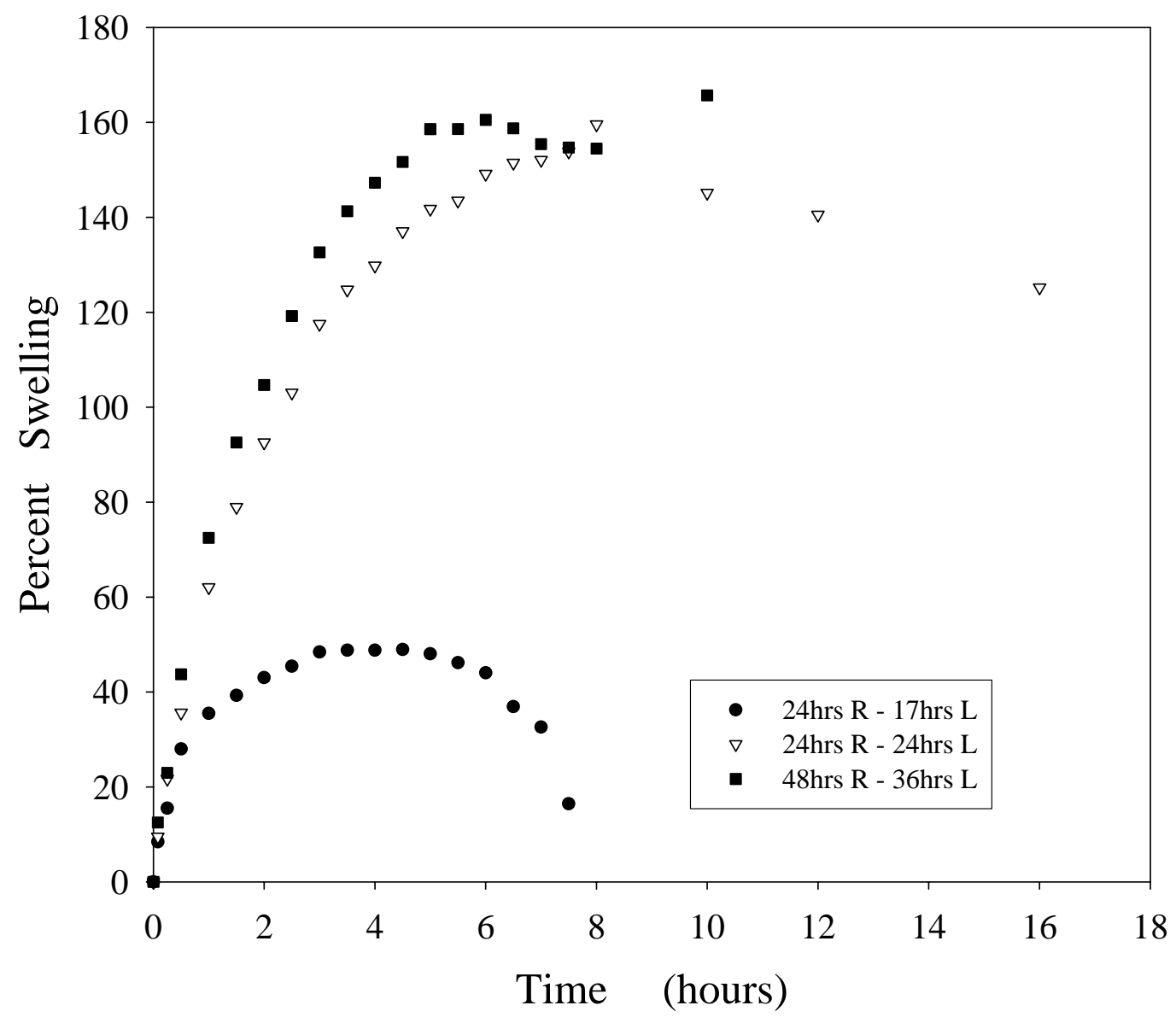

Figure 33. Swelling and erosion profiles of gelatin/15.8\% fructose matrices refrigerated and lyophilized for short, medium, and long curing times.

Matrices were evaluated under limited moisture and physiological temperatures.

The results are plotted as an average of triplicate measurements. 
for gelatin/sucrose, but a change in swelling behavior for the non-cross-linked formulation and in erosion for the gelatin/fructose formulation. What is notable is that, for all three formulations, the medium and long curing conditions had a pronounced affect on matrix behavior when compared with the short curing conditions. While percent swelling increased (likely because the matrices were more highly dried), erosion time was significantly increased. However, when the medium versus long curing conditions results were compared, neither seem to have an advantage over the other in performance

Several parameters need to be considered when determining the optimized process (Table 6). Of the three processes evaluated, the lyophilized matrices demonstrated minimal swelling and prolonged erosion across all formulations. The lyophilization is also is much more efficient at drying the matrices than either refrigeration or vacuum drying, requiring 2 days compared to 7 or 4 days, respectively

Figure 34 shows a comparison of swelling and erosion profiles for equal weight matrices before lyophilization to equal weight matrices after lyophilization. The weights of pre-lyophilized samples and post-lyophilized samples were $160.4 \pm 2.8 \mathrm{mg}$ and 165.87 $\pm 4.1 \mathrm{mg}$, respectively. In other words, for the equal weight pre-lyophilzed samples, all four formulations were cut into samples weighing from 155 to $165 \mathrm{mg}$ prior to the lyophilzation process. For the post-lyophilzation equal weight samples, the samples were cut so that all four formulations were of equal weight after the lyophilzation process was applied to the samples. Little to no difference is seen in each profile. This indicates that 
Table 6. Comparison of process effects of the optimized formulations.

The vacuum dried and lyophilized samples were refrigerated for 24 hours prior to the application of the curing condition. Data represents a mean of 3 determinations.

\begin{tabular}{|c|c|c|c|}
\hline GELATIN & $\begin{array}{c}\text { Process Time } \\
\text { (Days) }\end{array}$ & $\begin{array}{c}\text { Maximum } \\
\text { Percent } \\
\text { Swelling }\end{array}$ & $\begin{array}{c}\text { Time to Erosion } \\
\text { (Hours) }\end{array}$ \\
\hline Refrigeration & 7 & 200 & 8 \\
\hline Vacuum & 4 & 400 & 6 \\
\hline Lyophilization & 2 & 400 & 12 \\
\hline
\end{tabular}

\begin{tabular}{|c|c|c|c|}
\hline $\begin{array}{c}\text { GELATIN/ } \\
\text { SUCROSE }\end{array}$ & $\begin{array}{c}\text { Process Time } \\
\text { (Days) }\end{array}$ & $\begin{array}{c}\text { Maximum } \\
\text { Percent } \\
\text { Swelling }\end{array}$ & $\begin{array}{c}\text { Time to Erosion } \\
\text { (Hours) }\end{array}$ \\
\hline Refrigeration & 7 & 60 & 4.5 \\
\hline Vacuum & 4 & 200 & 4 \\
\hline Lyophilization & 2 & 100 & 10 \\
\hline
\end{tabular}

\begin{tabular}{|c|c|c|c|}
\hline $\begin{array}{c}\text { GELATIN/ } \\
\text { FRUCTOSE }\end{array}$ & $\begin{array}{c}\text { Process Time } \\
\text { (Days) }\end{array}$ & $\begin{array}{c}\text { Maximum } \\
\text { Percent } \\
\text { Swelling }\end{array}$ & $\begin{array}{c}\text { Time to Erosion } \\
\text { (Hours) }\end{array}$ \\
\hline Refrigeration & 6 & 60 & 2.5 \\
\hline Vacuum & 4 & 300 & 4 \\
\hline Lyophilization & 2 & 150 & 16 \\
\hline
\end{tabular}




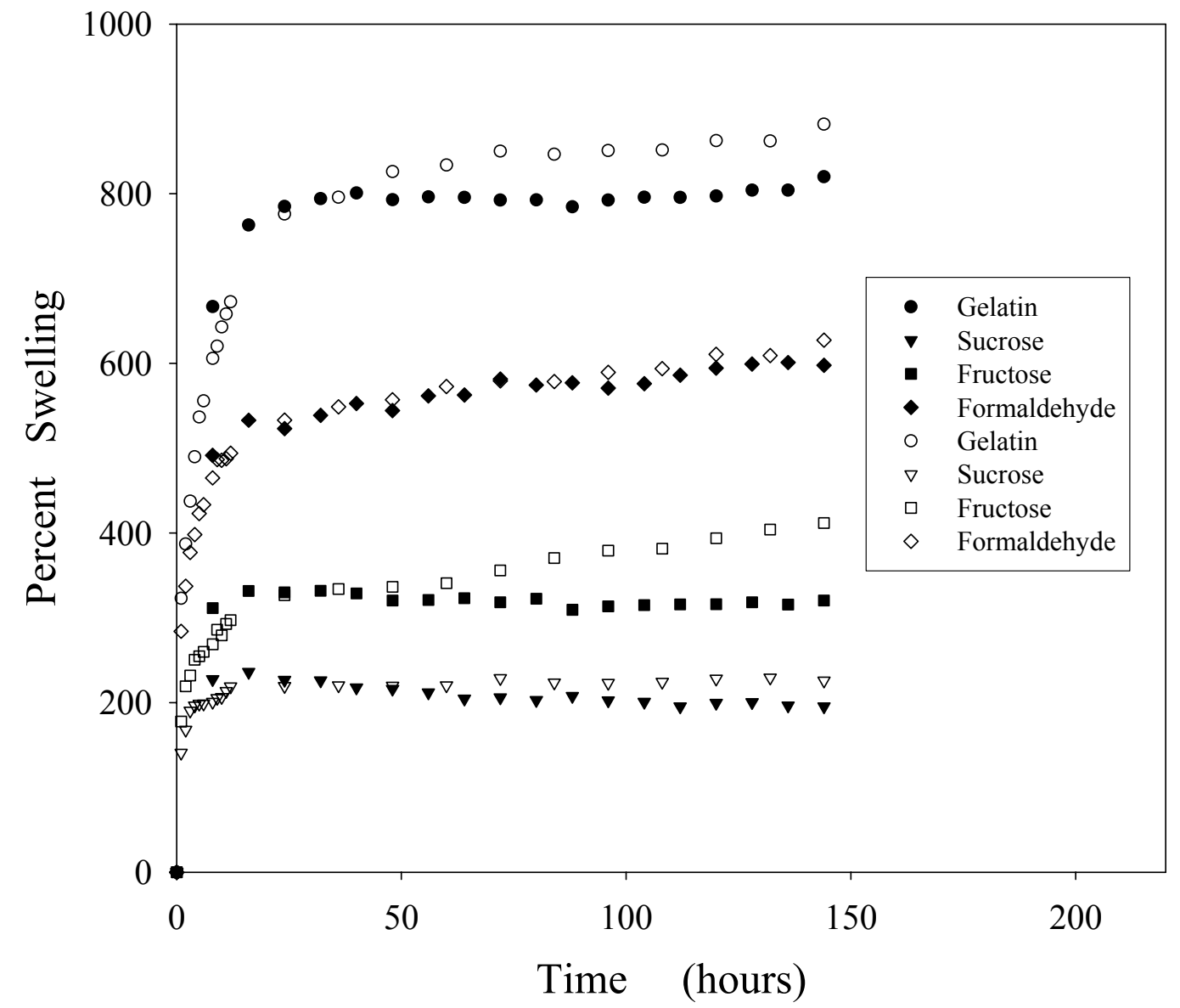

Figure 34. Swelling and erosion profile for equal weight samples pre- and postlyophilization.

The filled symbols represent pre-lyophilized samples with the same weight. The open symbols represent post-lyophilized samples with the same weight. Matrices were refrigerated for 24 hours followed by 24 hours of lyophilization and evaluated under excess moisture and ambient temperatures. The results are plotted as an average of triplicate measurements. 
the performance of the matrices is likely due to the extent of cross-linking within the matrix.

\section{Discussion}

After evaluating the two casting parameters, the 20 gauge needle and a casting temperature of $80^{\circ} \mathrm{C}$ produced the optimal casting conditions. Future studies incorporated these optimized conditions into the manufacturing process.

The refrigeration process was extremely limited in its ability to completely dry the disks. It required a minimum of 6 days of curing for the matrices to be strong and stable enough to demonstrate a desirable swelling/erosion profile. The vacuum drying process decreased the processing time from 6 days, for refrigeration, to 4 days but still required a long processing time in order to create a strong and stable matrix.

The small improvements seen when combining the refrigeration and vacuum drying processes have led to the evaluation of another drying process, lyophilization. The lyophilization process is much more efficient at drying the matrices ( 2 day curing time) than either refrigeration (6-7 day curing time) or vacuum drying (a 4 day curing time). Overall, the lyophilization process improved the performance of the gelatin and gelatin/sugar matrices. All formulations exhibited longer erosion times, up to 30 hours, compared to the 4-8 hours as seen with refrigeration alone.

The improvement in the performance of the matrices cured at room temperature, (data not shown) when compared to refrigeration or vacuum drying alone is due to the 
slow loss of water and an increase in the number of crystallites formed within the matrix. As the water is slowly removed from the matrix, small pores are formed within the matrix. The curing time, as well as small pores, allow the gelatin and/or cross-linking agents to be in closer proximity to each other as compared to the vacuum dried disks (a more rapid drying method), increasing the probability of physical or chemical interactions. (Further conformation of the chemical interactions utilizing solid-state NMR can be found in Appendix C). The small pore size offers an advantage over vacuum drying in that, upon swelling, a lower more limited volume of solvent is able to diffuse through the pores leading to a decrease in diffusion and drug release. In order to determine optimized curing conditions, the two curing variables (refrigeration and lyophilization) were evaluated over a range of values.

The influence of the various curing conditions on matrix structure was also confirmed through thermal analysis. After comparing multiple refrigeration and lyophilization times, the swelling and erosion profiles of the combination of 24 hours refrigeration followed by 24 hours of lyophilization best fits the ideal profile of an initial swelling phase followed by a plateau.

Based on the results of both formulation and process optimization studies reported in Chapters III and IV, films for future studies in this work were developed using the following conditions: $15 \%$ non-cross-linked gelatin, $15 \%$ gelatin/30\% sucrose, and $15 \%$ gelatin/15.8\% fructose matrices cured for 24 hours under refrigerated conditions followed by 24 hours of lyophilization. 


\section{References}

1. Tewari R, Crout R, Meyer-Stout PJ. Evaluation of a gelatin based controlled release drug delivery system for the treatment of periodontal disease, M.S. Thesis, WVU School of Pharmacy, 1997.

2. Levine H, Slade L. Water as a plasticizer: physiochemical aspects of low-moisture polymeric systems. In: Franks R, editor. Water Science Review. New York:CUP, 1998. p. 79-185.

3. Kumagai H, Sugiyama T, Iwamoto S. Effect of water content on dielectric relaxation of gelatin in a glassy state. J Agric Food Chem 2000;48:2260-5.

4. Levine H, Slade L. The glassy state of applications for the food industry, with an emphasis on cookie and cracker production. In: Blanshard JMV, Lillford PJ, editors. The Glassy State of Foods. Leicestershire, UK:NUP, 1993.

5. http://www.esb.ucp.pt/ bungah/lyo/sect3.htm

6. Martin A, Swarbrick J, Cammarata A. In: Physical Pharmacy. Philadelphia, PA:Lea \& Febiger, 1983. p532-533.

7. Clark RC, Courts A. The Chemical Reactivity of Gelatin. In: Ward AG, Courts A, editors. The Science and Technology of Gelatin. New York:Academic Press,1977. p. 212.

8. Baumgartner S, Kristl J, Peppas NA. Network structure of cellulose ethers used in pharmaceutical applications during swelling and at equilibrium. Pharm Res 2002;19(8):1084-1090. 


\section{Chapter V}

\section{Comparison of Optimized Novel Gelatin/Sugar Matrices with Traditional Gelatin/Formaldehyde Matrices}

The aldehydes have been used extensively as cross-linking agents in the preparation of bioprotheses, in cell and enzyme immobilization, and in protein and polysaccharide stabilization [1]. Bachet, et al [2] evaluated the effectiveness of a gelatinresorcinol-formaldehyde glue that could be used to reinforce tissues in the treatment of type A acute aortic dissection. The two most commonly studied aldehydes in the area of pharmaceutics are formaldehyde and glutaraldehyde. A gelatin microsphere cross-linked with D,L-glyceraldehyde was developed by Vandelli, et al [3] as a potential subcutaneous delivery system. The commercial gelatin product, Gelfoam ${ }^{\circledR}$, used for surgical packing as a hemostat, is also strengthened for slow erosion through formaldehyde as its crosslinking agent.

Formaldehyde, $\mathrm{H}_{2} \mathrm{C}=\mathrm{O}$, is the simplest aldehyde. The proposed mechanisms for gelatin/formaldehyde cross-linking can occur by one of two reactions as reported by Digenis, et al [4]. The first mechanism involves a reaction of the aldehyde with a lysyl $\varepsilon$ amino group forming a (hydroxymethyl) amino group. The (hydroxymethyl) amino group losses a water molecule forming an imine that can futher react with a (hydroxymethyl) amino group to form a dimethylene ether, rearrange, and form a methylene link. A second possible mechanism involves the reaction of the aldehyde with a lysyl $\varepsilon$-amino group forming a hemiaminal. The hemiaminal eliminates a water 
molecule, forming a cationic imine. The cationic imine can then further react with a free amine to form an aminal.

Thermal analysis studies, supported by data from matrix erosion studies, will be used to compare structural features as well as the performance characteristics of the sugar formulated matrices to aldehyde-based formulations.

\section{Methodology}

Materials

Gelatin disks were prepared using gelatin Type B 225 Bloom and D(-)-fructose purchased from Sigma (Sigma Chemical Co, St. Louis, MO). D(+)-sucrose was purchased from Fluka Chemical Co., Milwaukee, WI. Formaldehyde, $37 \mathrm{wt} \%$ solution in water, was purchased from Aldrich Chemical Co., Milwaukee, WI.

\section{Preparation of Gelatin Matrices}

Preparation of sugar cross-linked gelatin disks - Optimized Formulation

Gelatin/sugar disks were prepared by adding a $1 \mathrm{ml}$ aqueous solution containing

$632.5 \mathrm{mg}$ of fructose or $1200 \mathrm{mg}$ of sucrose to $3 \mathrm{ml}$ of a $20 \%(\mathrm{w} / \mathrm{v})$ gelatin solution (resulting in a final concentration of $15 \%$ ). The reaction mixture was heated to $80^{\circ} \mathrm{C}$ and maintained at the temperature for 5 minutes. The gelatin solution was syringed using a syringe into Teflon coated pans forming a circular gelatin disk. The disks were desiccated for 24 hours at refrigerated temperatures then lyophilized for an additional 24 hours. 


\section{Preparation of formaldehyde cross-linked gelatin disks}

The gelatin disks were prepared by heating a $15 \% \mathrm{w} / \mathrm{v}$ aqueous gelatin solution to

$50^{\circ} \mathrm{C}$. The formaldehyde was added at varying concentrations (ranging from 0.0185 to $0.148 \%$ ) to the solution followed by additional heating to $80^{\circ} \mathrm{C}$. The reaction mixture was maintained at $80^{\circ} \mathrm{C}$ for 5 minutes. Gelatin matrices were obtained as described above.

\section{Evaluation of swelling and bioerosion}

The swelling and erosion studies were conducted at $36 \pm 1^{\circ} \mathrm{C}$ in a Boekel Oven (Boekel Scientific, Feastersville, PA). Four samples were cut from each formulation. The dry weights of samples were recorded and each sample was placed on a nutrient agar plate. The samples were pulled and weighed at 5, 15, and 30 minutes followed by measurements at 30-minute intervals for the first 8 hours of the study. Samples were then weighed at 10,12, 16 and 24 hours, followed by measurements every 24 hours up to a pre-selected time or until the sample could no longer be weighed. Zero weight is the point at which the sample is no longer weighable. The percent swelling was calculated based on the following equation, $\%$ Swelling $=\left(\mathrm{m}_{\mathrm{t}}-\mathrm{m}_{\mathrm{i}}\right) / \mathrm{m}_{\mathrm{i}} \mathrm{x}$ 100, where $\mathrm{m}_{\mathrm{i}} \& \mathrm{~m}_{\mathrm{t}}$ are the weights (in $\mathrm{mg}$ ) of the initial sample and at time, $\mathrm{t}$, respectively.

\section{Thermal Characterization}

A piece of the sample was cut before and after drying to be analyzed using a Differential Scanning Calorimeter 7 (DSC) (PerkinElmer Instruments, Norwalk, CT). The sample was placed into an aluminum sample pan. The pan and sample were covered with a lid that was crimped so that the sample was encapsulated in the pan. The pan with the sample was placed in the sample holder, while an empty reference pan with a cover 
was placed on the reference side. The study was started after dry nitrogen was passed through the instrument for 10 minutes. A scanning rate of $20^{\circ} \mathrm{C}$ was evaluated in the temperature range of 25 to $300^{\circ} \mathrm{C}$.

\section{Experimental Design for Cross-linking Agent Comparison Studies}

Gelatin matrices were formulated over a range of formaldehyde concentrations, 0.0185 to $0.148 \%$, and performance was evaluated in order to determine optimal concentrations of formaldehyde. The basis for the selection of these concentrations was from prior work done in the laboratory [5], where formaldehyde was shown to strengthen gelatin-based matrices for dental drug sustained release delivery applications. The

optimal concentration is a concentration in which maximum cross-linking occurs between the gelatin and the formaldehyde (creating a strong, long-lasting matrix) without creating a film that becomes extremely brittle which would be difficult to work with in a clinical setting. Swelling and erosion studies were conducted to compare the optimized gelatin/sugar formulations to the gelatin/formaldehyde formulations. Thermal analysis studies were conducted to confirm strength and stability of the matrices. Performance studies were conducted to assess whether inert, native sugars can be used in place of the aldehydes to achieve the necessary design criteria for a sustained release drug delivery system.

\section{Results}

As with the non-cross-linked gelatin and gelatin/sugar matrices, the physical texture of the gelatin/formaldehyde matrices were followed over several days of curing. Upon congealing, the disks were transparent with a tint of yellow-brown. As the disks dried, they became extremely brittle. The more concentrated formaldehyde disks were 
extremely dry and offered the greatest resistance when compared to the other formaldehyde formulations as well as non-cross-linked gelatin and gelatin/sugar matrices when cutting them into smaller samples. This may be due to formaldehyde's ability to pull the gelatin strands closer together, serving as an effective cross-linking agent. When comparing the size and shape of the different cross-linking agents, formaldehyde is smaller in size and shape allowing the gelatin strands to be closer in proximity to each other than the strands would be in the sugar cross-linked matrices.

Similar to the gelatin and gelatin/sugar matrices, gelatin/formaldehyde matrices were first followed over a 7 day refrigeration period. The gelatin/formaldehyde matrices were jelly-like after 24 hours, but became stiff and brittle after 48 hours. The two concentrations of formaldehyde, $0.074 \%$ and $0.148 \%$, demonstrated different curing patterns. The lower concentration of formaldehyde, $0.074 \%$, exhibited a low percent swelling and prolonged erosion at 48 hours (Figure 35). At 72 hours, the matrices demonstrated a 2-fold increase in swelling and 3 hour longer erosion time than the matrices cured for 48 hours. However, little to no differences were seen between matrices cured for greater than 72 hours.

Gelatin matrices cross-linked with $0.148 \%$ formaldehyde demonstrated a different swelling and erosion pattern over the 7 day refrigeration period (Figure 36). After 24 hours of refrigeration, the matrices demonstrated minimal swelling and a moderate erosion time. At 48 hours, the matrices had a 2-fold increase in swelling and a similar 


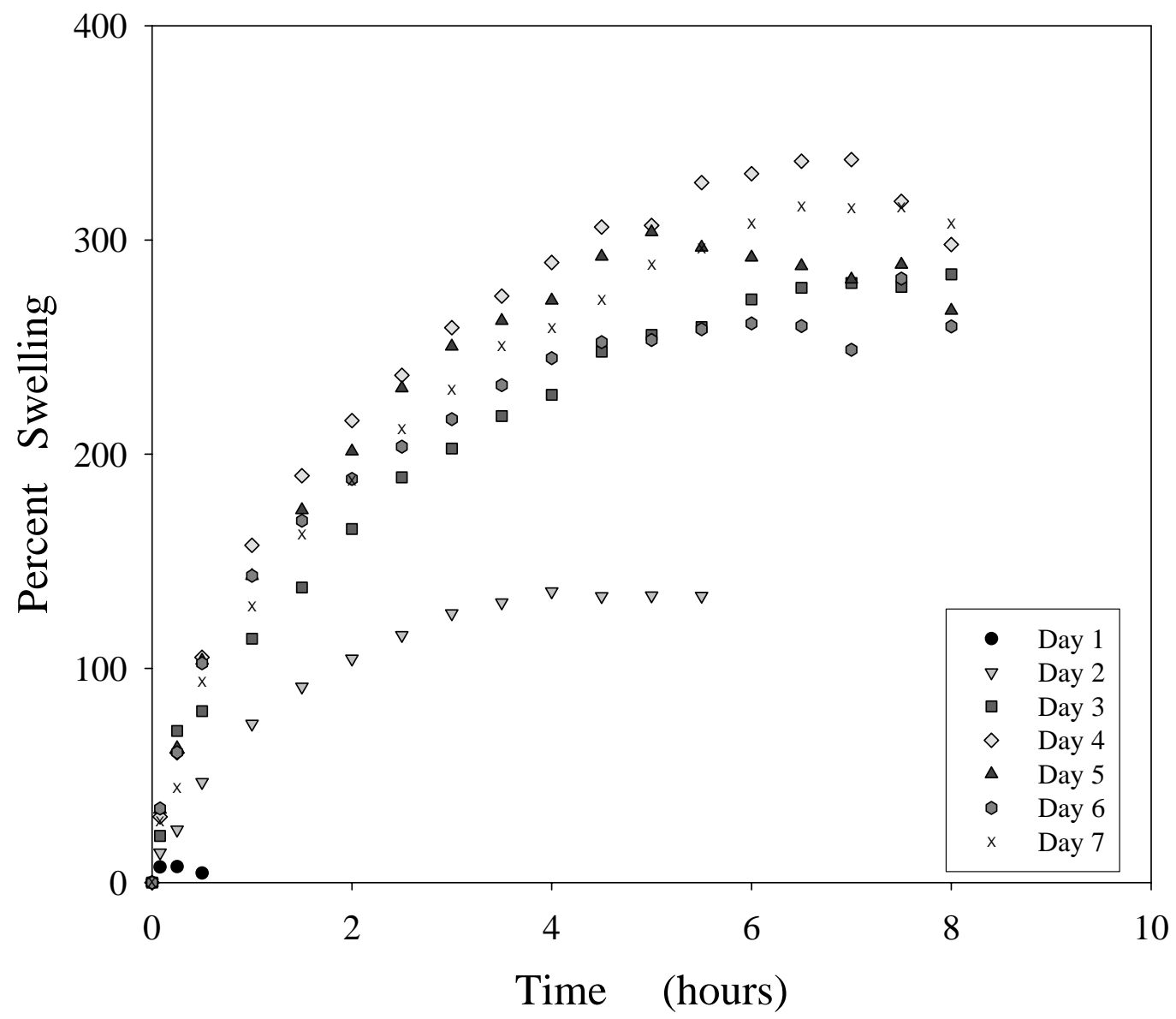

Figure 35. Swelling and erosion profile of gelatin/ $\mathbf{0 . 0 7 4 \%}$ formaldehyde matrices over a 7 day refrigeration period.

Matrices were evaluated under limited moisture and physiological temperatures.

The results are plotted as an average of triplicate measurements. 


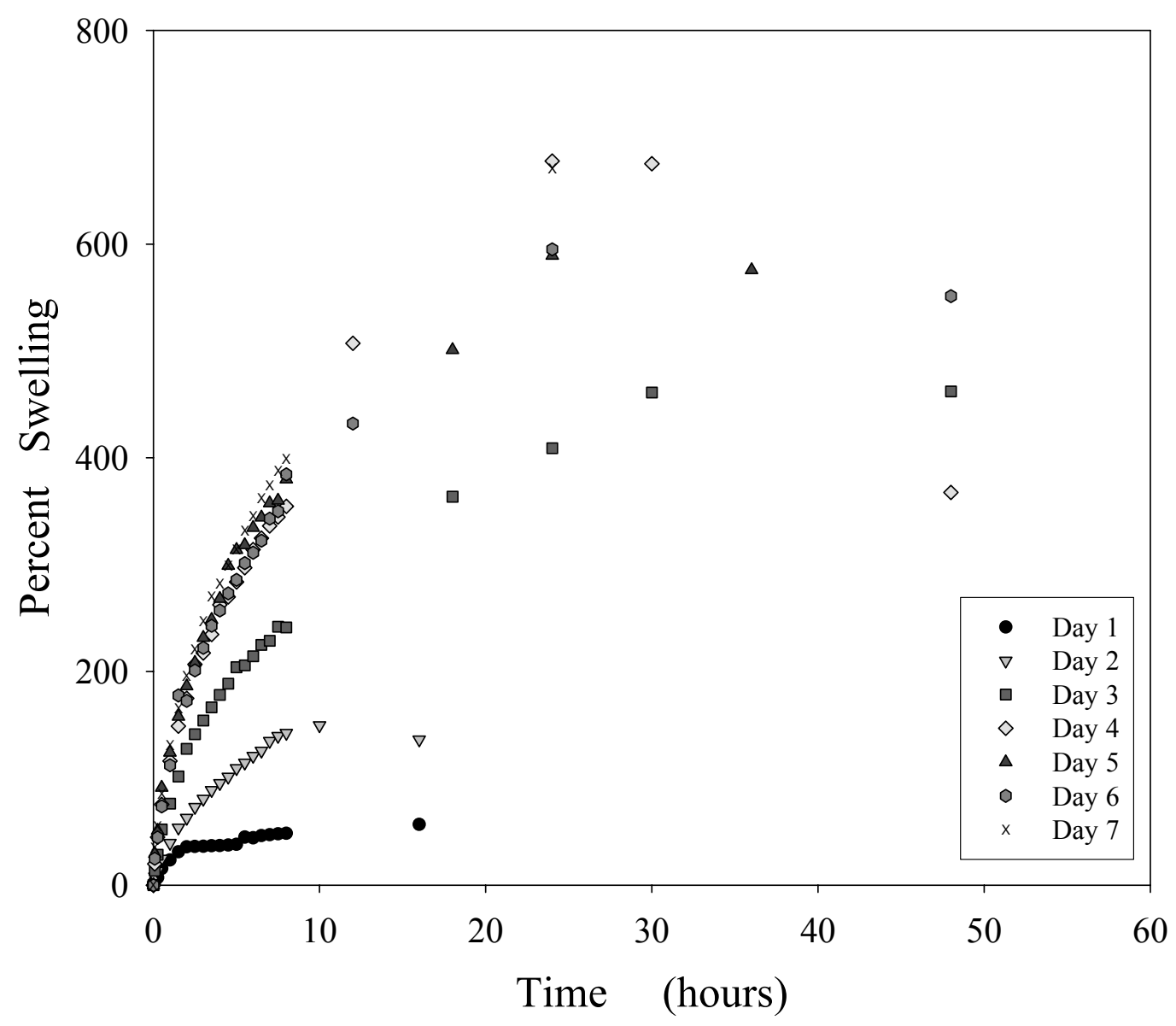

Figure 36. Swelling and erosion profile of gelatin/0.148\% formaldehyde matrices over a 7 day refrigeration period.

Matrices were evaluated under limited moisture and physiological temperatures.

The results are plotted as an average of triplicate measurements. 
erosion time to the matrices cured for 24 hours. An 8-fold increase in percent swelling and a 3-fold increase in erosion time were seen with matrices cured for 72 hours when compared to those cured for 24 hours. All matrices cured for longer than 72 hours demonstrated extensive swelling and prolonged erosion.

The extensive swelling seen with the gelatin/formaldehyde matrices is due to the tight network that is formed between the formaldehyde and the gelatin strands, likely forcing out any excess water embedded in the matrix. When exposed to solvent the matrix will thus take up water at a rapid rate throughout the time it is exposed to the solvent. Eventually, the matrix takes up enough water that it can no longer remain intact and disintegrates. As the swelling process occurs, the variability between samples is small and remains that way throughout the studies. This is due to the fact that the crosslinks formed between the formaldehyde and the gelatin likely stay intact and are extremely difficult to break. This may be due to the small size of the formaldehyde that brings the gelatin strands closer together, enabling them to interact at more sites along the chain through hydrogen bonds.

Gelatin/formaldehyde matrices formulated over a wide range of concentrations, $0.0185 \%$ to $0.074 \%$ final concentration, refrigerated for 24 hours followed by 24 hours of lyophilization were evaluated for swelling and erosion performance to determine the optimal concentration (Figure 37). Little to no differences were seen between the three concentrations of formaldehyde and the non-cross-linked gelatin matrices during the first 6 hours of the swelling phase. After 6 hours, pronounced differences, as manifested by 


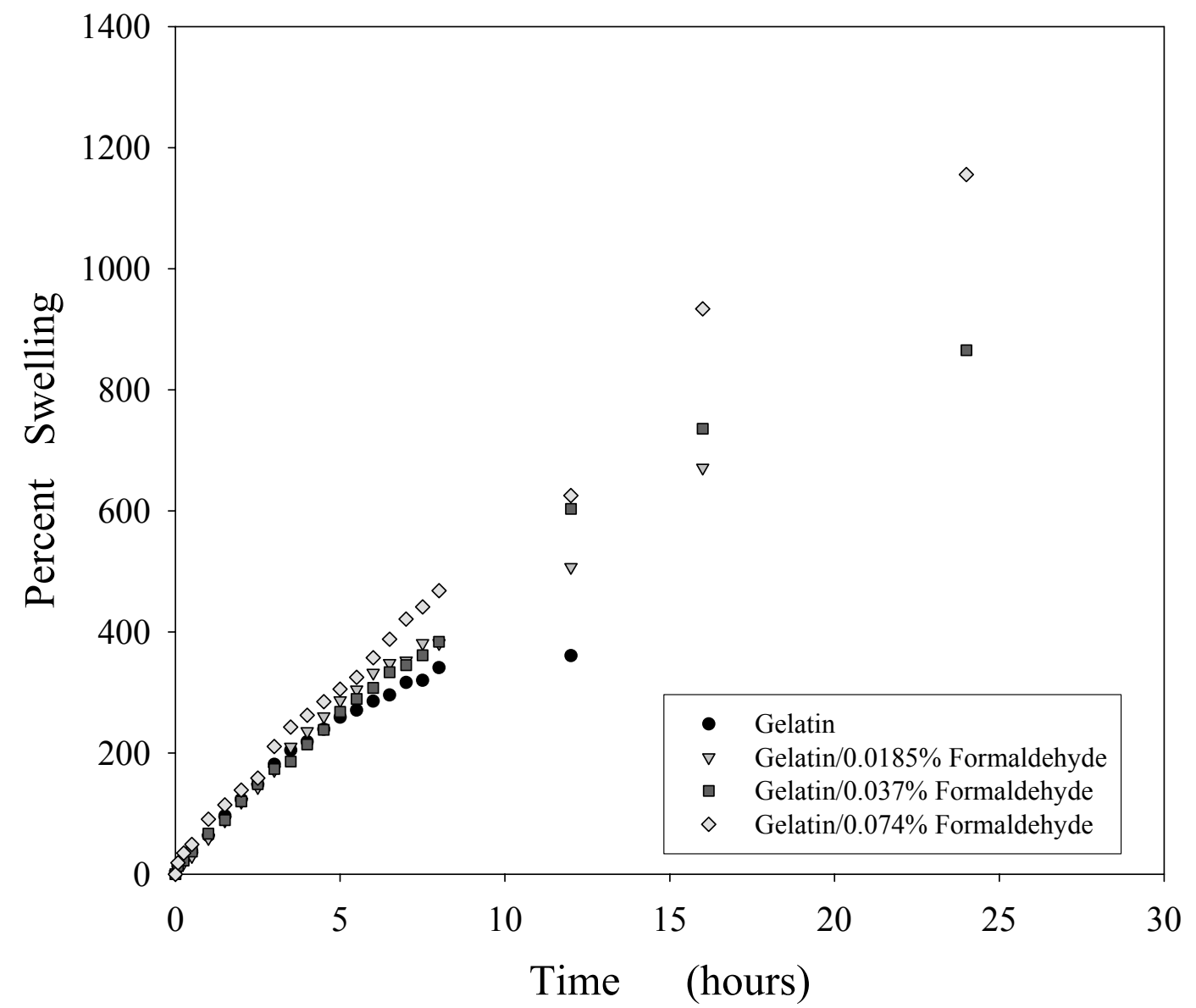

Figure 37. Swelling and erosion profile of varying concentrations of formaldehyde in gelatin/formaldehyde matrices.

Matrices were refrigerated for 24 hours followed by 24 hours of lyophilization and evaluated under limited moisture and physiological temperatures. The results are plotted as an average of triplicate measurements. 
an increased extent of swelling and prolonged time to erosion, are seen with increasing concentrations of formaldehyde. Of the three formaldehyde formulations investigated, the gelatin matrices cross-linked with $0.037 \%$ formaldehyde demonstrated the best swelling and erosion profile. The gelatin $/ 0.037 \%$ formaldehyde matrices exhibited the least amount of swelling and an equivalent erosion time when compared to the higher concentrations. When compared to the lower concentration formaldehyde cross-liked matrices, the gelatin $/ 0.037 \%$ formaldehyde matrices exhibited a longer erosion time while maintaining a similar swelling profile.

Thermal analysis data shows two endothermic peaks in the gelatin/formaldehyde spectrum (Figure 38). A peak at $80-90^{\circ} \mathrm{C}$ was seen indicating the presence of excess water within the matrix. As the matrix dries, this peak diminishes and eventually disappears after 3 days. A second peak appears between $160-220^{\circ} \mathrm{C}$ representing the gelatin/formaldehyde matrix transition to a liquid state, $\mathrm{T}_{\mathrm{m}}$. The gelatin/formaldehyde peaks are shifted to slightly higher temperatures compared to gelatin matrices demonstrating the increased strength and structural stability of the cross-linked matrices. The gelatin/formaldehyde matrices had similar profiles due to the low concentrations of formaldehyde in the matrices (data not shown).

Based on the results of both formulation and process optimization studies reported above, films for future studies in this work were developed using a $15 \%$ gelatin $/ 0.037 \%$ formaldehyde matrix cured for 24 hours under refrigerated conditions followed by 24 hours of lyophilization. 


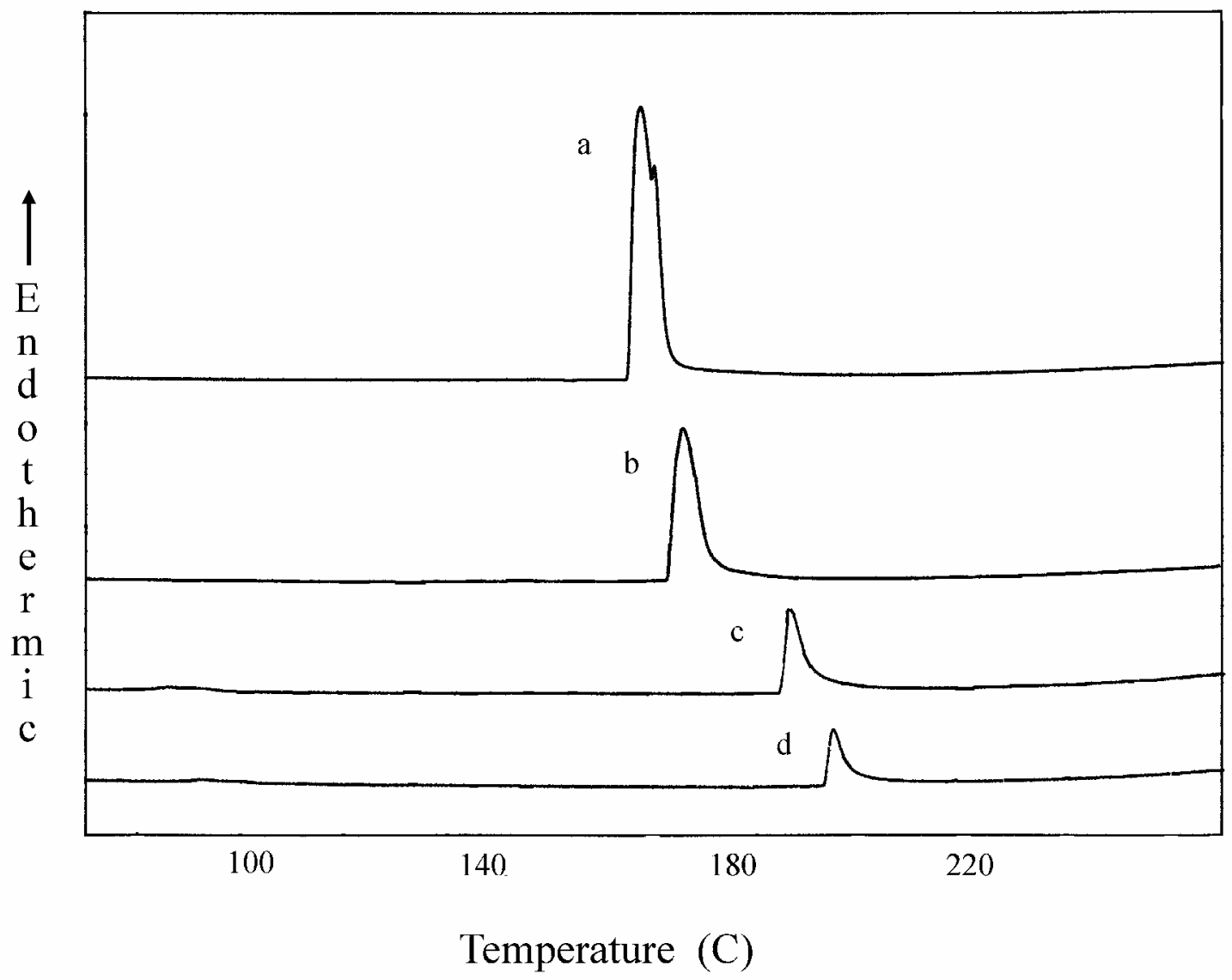

Figure 38. Thermal analysis scans of the gelatin/0.037\% formaldehyde matrix. Lines a-d represent the first four days of the refrigeration process. 
In order to evaluate the overall performance of the gelatin/sugar matrices, the optimized sugar cross-linked gelatin matrices (30\% sucrose and 15.8\% fructose concentrations) were compared to the optimized formaldehyde cross-linked gelatin matrix ( $0.037 \%$ formaldehyde). All formulations were refrigerated for 24 hours followed by 24 hours of lyophilization. The gelatin/formaldehyde matrices become swollen to a larger extent than the gelatin/sugar matrices but exhibited prolonged erosion (Figure 39). Upon hydration, the gelatin/sugar matrices doubled their original weight while the noncross-linked matrices demonstrated a 4.5 fold increase and the gelatin/formaldehyde a 9 fold increase in their original weight. The gelatin/formaldehyde matrices swell to a greater extent than the other three formulations because the gelatin/formaldehyde matrices are most likely in the swelling phase during the first 24 hours of the study. In Figure 39, during the first 7 hours of the profile, the non-cross-linked gelatin and gelatin/sugar matrices are predominantly in the swelling phase. Around 7-8 hours, the erosion phase becomes the more predominant phase and the matrices eventually dissolve. The gelatin/formaldehyde matrices exhibited a prolonged erosion of 24 hours compared to 16,12 , and 10 hours for gelatin/fructose, non-cross-linked gelatin, and gelatin/sucrose, respectively.

Thermal analysis data (Table 7) shows little to no difference between the melting temperatures of the four optimized formulations. The gelatin and gelatin/formaldehyde matrices demonstrated the same endothermic peak around $80-90^{\circ} \mathrm{C}$ which disappears during the curing process. 


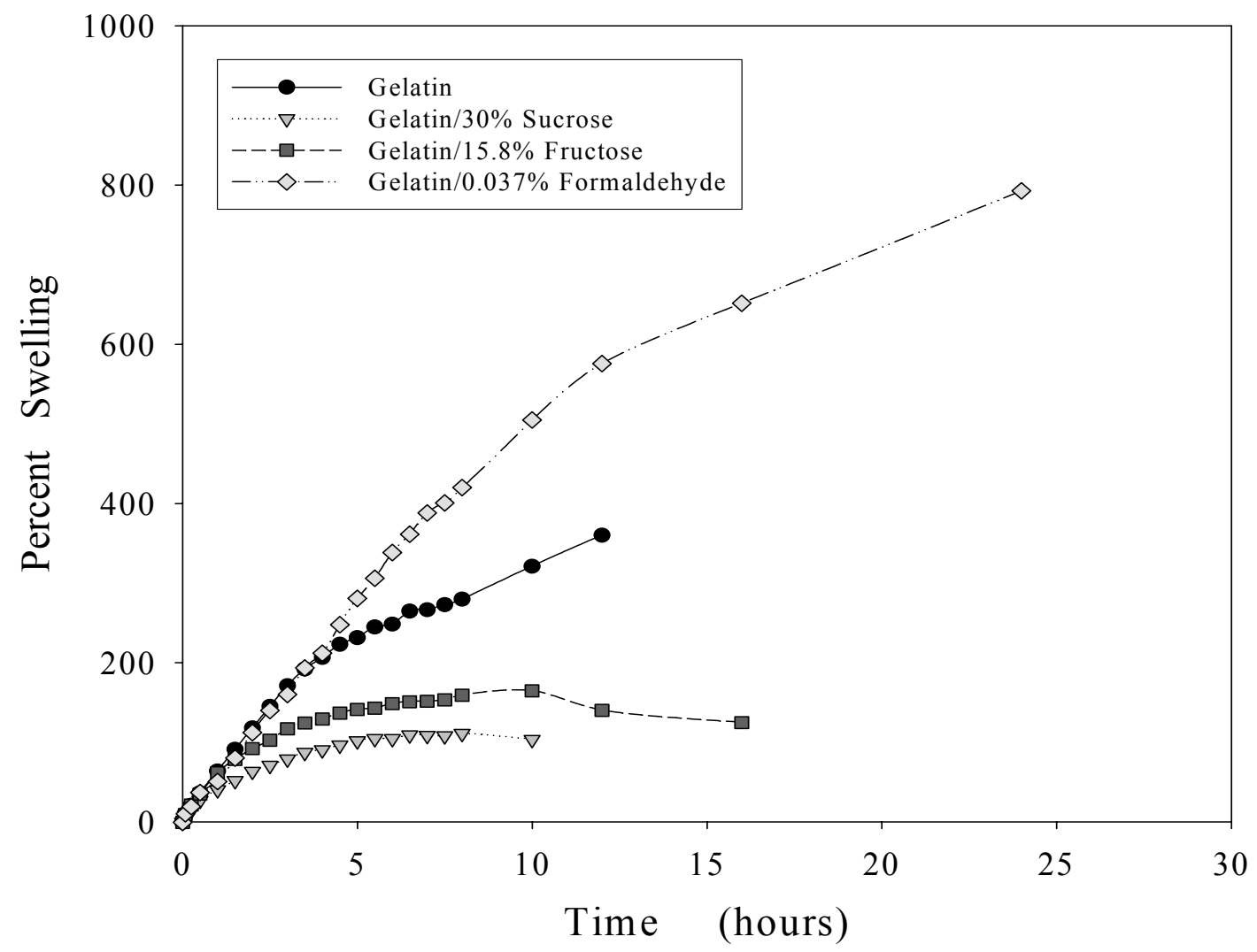

Figure 39. Swelling and erosion profile of gelatin, gelatin/sucrose, gelatin/fructose, and gelatin/formaldehyde.

Matrices were refrigerated for 24 hours followed by 24 hours of lyophilization and evaluated under limited moisture and physiological temperatures. The results are plotted as an average of triplicate measurements. 
Table 7. Comparison of $\mathbf{T}_{\mathbf{m}}$ among the five formulations.

Data presented here presents a compilation of thermal analysis data for the three curing processes. HCHO represents formaldehyde. Data represents a mean of 3 determinations.

\begin{tabular}{|c|c|c|}
\hline & $\begin{array}{c}\text { Temperature } \\
\text { Range }\left({ }^{\circ} \mathrm{C}\right)\end{array}$ & $\begin{array}{c}\text { Additional } \\
\text { Endothermic Peaks } \\
\left({ }^{\circ} \mathrm{C}\right)\end{array}$ \\
\hline Gelatin & $160-220$ & $90-100$ \\
\hline Gelatin/Sucrose & $170-220$ & 220 \\
\hline Gelatin/Fructose & $170-220$ & --- \\
\hline Gelatin/0.037\% HCHO & $160-220$ & $80-90$ \\
\hline Gelatin/0.074\% HCHO & $160-220$ & $80-90$ \\
\hline
\end{tabular}




\section{Discussion}

As seen with the gelatin/sugar matrices, the refrigeration and vacuum drying processes were efficient in removing water from the matrices but required long curing times (5-7 days and 4 days, respectively). The addition of the lyophilization process improved the performance of the gelatin/formaldehyde matrices. When comparing the various formulation and process conditions, gelatin matrices loaded with $0.037 \%$ formaldehyde show better swelling and erosion characteristics for sustained drug delivery, lasting twice as long as the non-cross-linked gelatin matrices. Based on the results of both formulation and process optimization studies, matrices for future studies in this work were developed using a $15 \%$ gelatin $/ 0.037 \%$ formaldehyde matrix that was refrigerated for 24 hours then lyophilized for 24 hours.

Comparison of the four formulations (gelatin, gelatin/30\% sucrose, gelatin/15.8\% fructose, and gelatin $/ 0.037 \%$ formaldehyde) shows a gelatin/formaldehyde formulation with a prolonged erosion and large extent of swelling. Although the formaldehyde loaded films exhibit prolonged erosion, 24 hours versus 16 hours for gelatin/fructose, the gelatin/sugar matrices exhibit more desirable swelling characteristics, low percent swelling with a prolonged plateau. The gelatin/formaldehyde and non-cross-linked gelatin matrices never reach the plateau before absorbing a sufficient quantity of solvent to cause disintegration of the matrices. This is important because extent and rate of swelling may impact the release characteristics of the drug release. The ultimate significance of these parameters will be determined from drug release studies (Chapter VI). 
For the purposes of this work, the ideal swelling/erosion profile shows a slow initial swelling followed by a prolonged plateau and erosion. Of the four formulations evaluated in Chapters III-V, the gelatin/sugar matrices (30\% sucrose and $15.8 \%$ fructose, final concentrations), refrigerated for 24 hours then lyophilized for 24 hours, best fit this profile, absorbing a sufficient quantity of solvent to cause dissolution of the matrices. Initial studies have shown that sucrose formulation appeared to be a more effective crosslinking agent. However, after applying the three curing methods to the formulations, both sucrose and fructose appear to be viable cross-linking agents. 


\section{References}

1. Ulubayran K, Aksu E, Gurhan SID, Serbetci K, Hasirci N. Cytotoxicity evaluation of gelatin sponges prepared with different cross-linking agents. J Biomater Sci Polymer Edn 2002;13(11):1203-1219.

2. Bachet J, Goudot B, Teodori G, Brodaty D, Dubois C, DeLentdecker P, Guilmet D. Surgery of type A acute aortic dissection with gelatin-resorcinol-formal biological glue: a twelve-year experience. J Cardiovasc Surg 1990;31:263-273.

3. Vandelli MA, Rivasi R, GuerraP, Forni F, Arletti R. Gelatin microspheres crosslinked with D,L-glyceraldehyde as a potential drug delivery system: preparation, characterisation, in vitro and in vivo studies. Int J Pharm 2001;215:175-184.

4. Digenis GA, Gold TB, Shah VP. Cross-linking of gelatin capsules and its relevance to their in vitro-in vivo performance. J Pharm Sci 1994;83:915-21.

5. Tewari R, Crout R, Meyer-Stout PJ. Evaluation of a gelatin based controlled release drug delivery system for the treatment of periodontal disease, M.S. Thesis, WVU School of Pharmacy, 1997. 


\section{Chapter VI}

\section{Drug Release Characteristics}

The release profile and release kinetics has been extensively studied from gelatin and collagen matrices. Chowdhury, et al [1] reported that the release of deoxyuridine from cross-linked collagen and collagen-gelatin matrices follows the Higuchi square root of time equation. The authors have also reported that the solubility of the drug may affect the release characteristics. Leucuța, et al [2] has shown that the release of nifedipine from gelatin microspheres or microcapsules follows either a bi-expontential first-order or square root equation. The use of gelatin and collagen for tissue engineering has increased due to biocompatibility with soft tissue. Choi, et al [3] developed novel gelatin-alginate sponges that could be loaded with drug for use as tissue engineering scaffolds. The authors evaluated drug bioavailability studies and the morphology of the sponges. Hydrogels are polymers that have the ability to swell in aqueous solvent systems and retain a significant fraction of water within their molecular structure [4]. Changez, et al [5] evaluated the release of gentamicin sulphate from poly(acrylic acid)gelatin hydrogels. The authors conclude that gentamicin release demonstrates a biphasic pattern and is dependent on the composition of the hydrogel as well as the $\mathrm{pH}$ of the medium.

This phase assessed the drug release characteristics of the four optimized formulations. The optimized formulations evaluated in this phase are gelatin, gelatin $/ 30 \%$ sucrose, gelatin $/ 15.8 \%$ fructose, and gelatin $/ 0.037 \%$ formaldehyde that were refrigerated for 24 hours, then lyophilized for an additional 24 hours. Qualitative studies 
using food coloring were conducted to visually assess the release characteristics of a one or two drug component/gelatin matrix system. Quantitative studies then followed to characterize the drug release and identify the release model and/or controlling mechanisms for release.

\section{Methodology}

\section{Materials}

Gelatin disks were prepared using gelatin Type B 225 Bloom and D(-)-fructose purchased from Sigma (Sigma Chemical Co, St. Louis, MO). D(+)-sucrose was

purchased from Fluka Chemical Co., Milwaukee, WI. Formaldehyde, $37 \mathrm{wt} \%$ solution in water, and fluorescein sodium was purchased from Aldrich Chemical Co., Milwaukee, WI. The red and green dye are manufactured by McCormick \& Co, Inc (Hunt Valley, MD).

\section{Color Studies - Qualitative}

Dye loaded films were formulated by incorporating green and/or red dye into the four optimized formulations identified from earlier studies (Chapters III-IV). The red dye consists of two non-natural compounds, Allura Red AC and Erythrosine (Figure 40), certifiable for use in the United States by the Food and Drug Administration. Allura Red $\mathrm{AC}(\mathrm{MW}=497)$ is an orange-red dye and Erythrosine $(\mathrm{MW}=880)$ is a cherry red dye. The red dye has chromophores which are detectable in the visible range at $504 \mathrm{~nm}$ and $525 \mathrm{~nm}$, respectively. The green dye consists of two non-natural compounds, Brilliant Blue FCF and Tartrazine (Figure 41), certifiable for use in the United States by the Food and Drug Administration. Brilliant Blue FCF (MW = 793) is a bright blue dye and Tartrazine $(\mathrm{MW}=534)$ is a lemon-yellow dye. The green dye has 


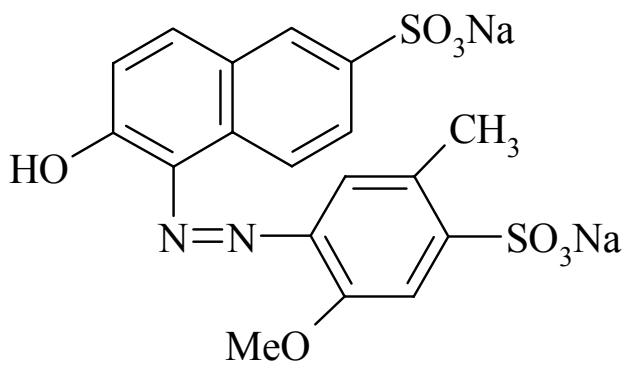

Allura Red AC

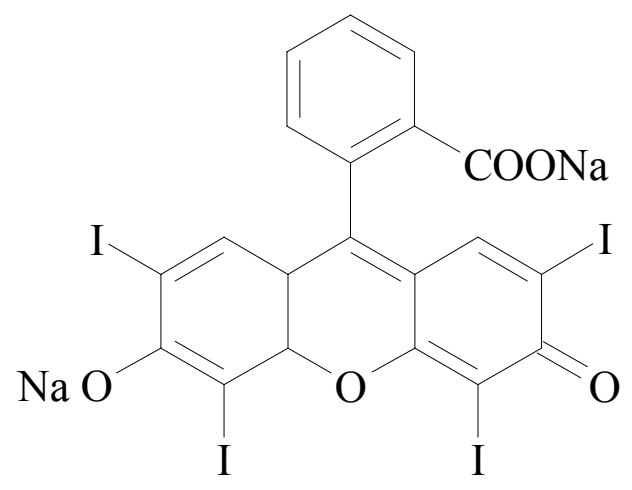

Erythrosine

Figure 40. Structures of Red Dye: Allura Red AC and Erythrosine. 


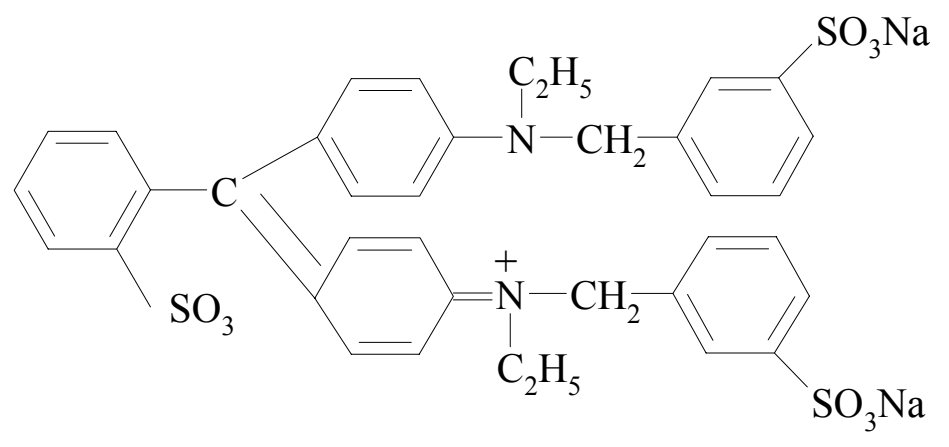

Brilliant Blue FCF

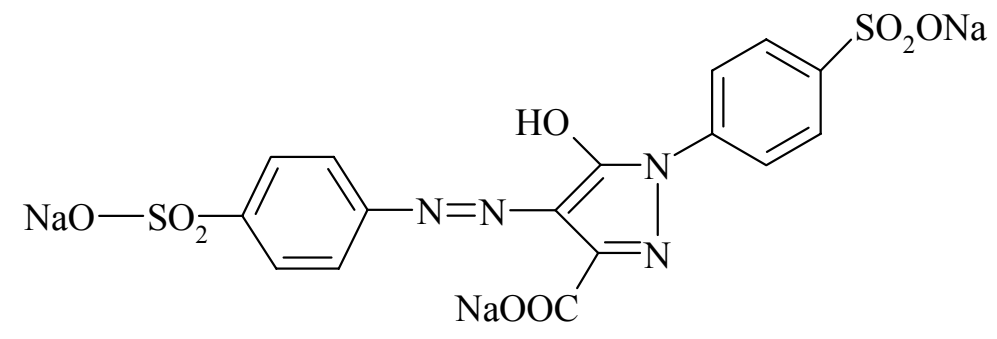

Tartrazine

Figure 41. Structures of green dye: Brilliant Blue FCF and Tartrazine. 
chromophores which are detectable in the visible range at $625 \mathrm{~nm}$ and $415 \mathrm{~nm}$, respectively. The four components of the red and green dye are sodium salts. Qualitative studies provided visual information about the release characteristics of the optimized formulations.

\section{Preparation of dye loaded non-cross-linked gelatin disks}

Gelatin disks were prepared by heating a $15 \% \mathrm{w} / \mathrm{v}$ aqueous gelatin solution to

$80^{\circ} \mathrm{C}$. During the heating process when the gelatin solution reached $50^{\circ} \mathrm{C}, 240 \mu 1$ of green and/or red dye was added to the solution. The reaction mixture was maintained at $80^{\circ} \mathrm{C}$ for 5 minutes and syringed using a syringe into Teflon coated wells, forming a circular disk. The disks were desiccated at refrigerated temperatures for 24 hours and lyophilized for an additional 24 hours. (NOTE: It should be noted that, any time a drug/drug prototype is added to any of the gelatin matrix formulations, the effect of the elevated temperature of the dispersion process on the drug and the potential to cause degradation was factored into the point (temperature), at which the drug is added.)

Preparation of dye loaded sugar cross-linked gelatin disks - Optimized Formulation

The gelatin disks were prepared by adding a $1 \mathrm{ml}$ aqueous solution containing $632.5 \mathrm{mg}$ or fructose or $1200 \mathrm{mg}$ of sucrose to $3 \mathrm{ml}$ of a $20 \%(\mathrm{w} / \mathrm{v})$ gelatin solution (resulting in a final concentration of $15 \%$ ). The reaction mixture was heated to $80^{\circ} \mathrm{C}$. During the heating process when the gelatin solution reached $50^{\circ} \mathrm{C}, 240 \mu \mathrm{l}$ of green and/or red dye was added to the solution. The reaction mixture was maintained at $80^{\circ} \mathrm{C}$ for 5 minutes. Gelatin matrices were obtained as described above. 


\section{Preparation of dye loaded formaldehyde cross-linked gelatin disks - Optimized}

Formulation

The gelatin disks were prepared by heating a $15 \% \mathrm{w} / \mathrm{v}$ aqueous gelatin solution to $80^{\circ} \mathrm{C}$. During the heating process when the gelatin solution reached $50^{\circ} \mathrm{C}$, a $0.1 \%(\mathrm{v} / \mathrm{v})$ volume of a $37 \%$ formaldehyde solution followed by $240 \mu \mathrm{l}$ of green and/or red dye were added to the solution. The reaction mixture was maintained at $80^{\circ} \mathrm{C}$ for 5 minutes. Gelatin matrices were obtained as described above.

\section{Visual Release Study - Qualitative}

Under wet conditions, color studies were conducted at room temperature. Dry weights were recorded (average weight for gelatin and gelatin/formaldehyde matrices was 30-50 mg, gelatin/fructose was 65-80 mg, and gelatin/sucrose was 90-120 mg) and each sample was placed in a test tube containing non-buffered, distilled water $(\mathrm{pH} 4.8$ 5.2), approximately 6 milliliters. The samples were pulled and weighed at 5, 15, and 30 minutes, followed by measurements at 30 minute intervals. After the first 24 hours, the films were weighed at varying intervals from every 24 hours to 5 days. The study was continued until the samples could no longer be weighed. The water in the test tube was changed at varying intervals as short as every hour up to every 5 days to minimize saturation of solution of gelatin. A number was assigned at each exchange to visually assess the amount of dye in the solution. The assigned numbers ranged from 0 to 5 with zero indicating no dye color present and a 5 indicating maximum dye color observed. The score assigned to each test tube is an individual and discrete score, not a cumulative number. Each color score assignment represented a discrete interval of time of dye release. These values were determined for internal laboratory purposes and not quantitative purposes. 


\section{Drug Loading Studies - Quantitative}

Since the laboratory has collaboration in the areas of periodontal and ocular drug delivery, the in vitro test conditions for quantitating drug release (in this case, dye release) were developed to mimic these delivery sites (limited fluid volume, high fluid turnover rates). Furthermore, another solute, fluorescein (Figure 42), was also included in these studies to serve as a drug marker. The addition of fluorescein to the gelatinbased matrix formulations occurred in the same manor as the dye loaded matrices which is described in disk preparation section (Section I - Color Studies - Quantitative) of this chapter. Fluorescein was added to the gelatin dispersion when the temperature reached $50^{\circ} \mathrm{C}$. Fluorescein offered the advantages of clinical use (ophthalmic), sensitive assay, and known solubility profile (which is not known for the dyes). Fluorescein is freely soluble in water [6]. 


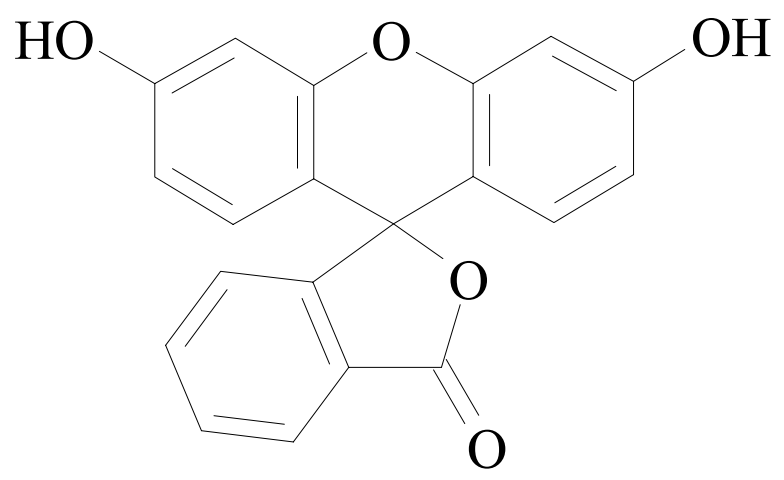

Figure 42. Structure of fluorescein. 


\section{Content Analysis of the Matrices}

The dye and fluorescein loaded matrices were placed in $6 \mathrm{ml}$ of non-buffered distilled water. The weight of the samples ranged from $30 \mathrm{mg}$ to $35 \mathrm{mg}$ for the gelatin and gelatin/formaldehyde matrices and $70 \mathrm{mg}$ to $100 \mathrm{mg}$ for the gelatin/sugar matrices. The samples were melted at $37^{\circ} \mathrm{C}$, filtered using a 0.47 micron filter, and assayed for the concentration by the methods described below. The total amount of dye loaded in each sample was calculated by converting absorbance to concentration (v/v) using the standard curves, found in Appendix B and C, then multiplying the concentration by the volume of the sample.

\section{In Vitro Dye Release}

Under limited wet conditions, release studies were conducted at room temperatures. A sample of each matrix was placed in a test tube containing $3 \mathrm{mls}$ of nonbuffered distilled water $(\mathrm{pH}=4.8-5.2)$. The solution was pulled at one hour intervals and analyzed spectrophotometrically (Beckman DU 65, Single Beam Spectrophotometer, Philadelphia, PA), by visible analysis. After 12 hours, the solution was analyzed every 12 hours for a minimum of 3 days and a maximum of 7 days. The release studies were evaluated under sink and non-sink conditions. In order to maintain sink conditions, the total volume $(3 \mathrm{ml})$ was replaced at each time interval while in non-sink conditions the volume remained the same and was not replaced at each time point. Sink conditions for these studies could not be absolutely assured since the exact concentration of each dye in the commercial product was unknown. The release results are plotted as the cumulative percentage of dye in the dissolution medium versus time. 
Standard curves were constructed using nine concentrations. The green dye was analyzed at two wavelengths, $415 \mathrm{~nm}$ and $625 \mathrm{~nm}$, while the red was analyzed at wavelengths of $504 \mathrm{~nm}$ and $525 \mathrm{~nm}$. Curves were obtained daily for multiple days by plotting the absorbance against the corresponding concentration. Standard curves for the green and red dye can be found in Appendix B. Total dye was determined by the summation of the two dye components. Due to the fact that each sample is hand-cut and resulting in different sample weights, the amount of dye loaded per mg of sample was calculated for each sample. The amount of dye/mg sample $=$ (total amount of dye loaded)/weight of each sample. The total amount of dye loaded in each sample was calculated by converting absorbance to concentration ( $\mathrm{v} / \mathrm{v})$ using the standard curves, found in Appendix B, then multiplying the concentration by the volume of the sample. The calculation of percent release is based on the following equation: $\%$ Release $=$ $\{($ concentration of green dye released $\mathrm{x} 3 \mathrm{ml})+($ concentration of red dye released $\mathrm{x}$ $3 \mathrm{ml} /($ average amount of dye/mg sample x sample weight) $\}$ x 100.

\section{In Vitro Drug Release}

A sample from each fluorescein-loaded matrix was placed in a test tube containing $3 \mathrm{mls}$ of non-buffered distilled water $(\mathrm{pH}=4.8-5.2)$ at ambient temperatures. At 4 hour intervals, the samples were removed from the medium, weighed, and placed in a new test tube containing fresh water, thus allowing for sink conditions. The solution was assayed using a fully automated Waters (Waters Co, Milford, MA) HPLC with a Zorbax ${ }^{\circledR}$ ODS Column (Agilent, Palo-Alto, CA). The mobile phase was a mix (60:40)

of methanol and water at a flow rate of $1 \mathrm{ml} / \mathrm{min}$ at ambient temperatures. The peak was 
detected by measuring absorbance at $485 \mathrm{~nm}$ using a photodiode array. The retention time was 3-3.5 minutes. Sample chromatograms can be found in Appendix C.

Standard curves were constructed using nine concentrations (ranging from $30 \mathrm{ng}$ to $3,000 \mathrm{ng}$ and $1 \mu \mathrm{g}$ to $500 \mu \mathrm{g}$ ) and analyzed at $485 \mathrm{~nm}$. Curves were obtained daily for multiple days by plotting the absorbance against the corresponding concentration. Standard curves for fluorescein can be found in Appendix C. Due to the fact that each sample is hand-cut and resulting in different sample weights, the amount of dye loaded per mg of sample was calculated for each sample. The amount of dye/mg sample $=$ (total amount of dye loaded)/weight of each sample. The total amount of dye loaded in each sample was calculated by converting absorbance to concentration (v/v) using the standard curves, found in Appendix B, then multiplying the concentration by the volume of the sample. The calculation of percent release is based on the following equation: \% Release $=($ concentration of green dye released $\mathrm{x} 3 \mathrm{ml}) /($ average amount of dye $/ \mathrm{mg}$ sample $\mathrm{x}$ sample weight) $\}$ x 100.

\section{Evaluation of swelling and bioerosion}

The swelling and erosion studies were conducted at $25 \pm 1{ }^{\circ} \mathrm{C}$. Four samples were cut from each formulation. The dry weights of samples were recorded and each sample was placed in a test tube containing non-buffered distilled water $(\mathrm{pH} 4.8-5.2)$. The samples were pulled, blotted with an absorbent wipe (Kimwipes, Kimberly-Clark, Roswell, GA), and weighed (Mettler Model AE50) at 5, 15, and 30 minutes, followed by measurements at 30-minute intervals until the sample could no longer be weighed or up to a designated time. Zero weight was the point at which the sample was no longer 
weighable. The percent swelling was calculated based on the following equation, $\%$ Swelling $=\left(\mathrm{m}_{\mathrm{t}}-\mathrm{m}_{\mathrm{i}}\right) / \mathrm{m}_{\mathrm{i}} \times 100$, where $\mathrm{m}_{\mathrm{i}} \& \mathrm{~m}_{\mathrm{t}}$ are the weights (in $\mathrm{mg}$ ) of the initial sample and at time, $t$, respectively.

\section{Thermal Characterization}

A piece of the sample was cut before and after drying to be analyzed using a Differential Scanning Calorimeter 7 (DSC) (PerkinElmer Instruments, Norwalk, CT). The sample was placed into an aluminum sample pan. The pan and sample were covered with a lid that was crimped so that the sample was encapsulated in the pan. The pan with the sample was placed in the sample holder, while an empty reference pan with a cover was placed on the reference side. The study was started after dry nitrogen was passed through the instrument for 10 minutes. A scanning rate of $20^{\circ} \mathrm{C}$ was evaluated in the temperature range of 25 to $300^{\circ} \mathrm{C}$.

\section{Results}

\section{Content Analysis of the Matrices}

The amount of green dye loaded in the films varied depending on the formulation. The non-cross-linked gelatin matrices contained $0.0115 \pm 0.0010 \mu \mathrm{l} / \mathrm{mg}$ of sample for the yellow component and $0.0157 \pm 0.0013 \mu \mathrm{l} / \mathrm{mg}$ of sample for the blue component. The gelatin/sucrose matrices contained $0.0090 \pm 0.0007 \mu \mathrm{l} / \mathrm{mg}$ of sample for the yellow component and $0.0124 \pm \mu 1 / \mathrm{mg}$ of sample for the blue component. The gelatin/fructose matrices contained $0.0083 \pm 0.0007 \mu \mathrm{l} / \mathrm{mg}$ of sample for the yellow component and $0.0115 \pm 0.0009 \mu \mathrm{l} / \mathrm{mg}$ of sample for the blue component. The gelatin/formaldehyde 
matrices contained $0.0131 \pm 0.0007 \mu \mathrm{l} / \mathrm{mg}$ of sample for the yellow component and $0.0158 \pm 0.0008 \mu \mathrm{l} / \mathrm{mg}$ of sample for the blue component.

The amount of red dye loaded in the films varied depending on the formulation. Unlike the matrices loaded with green dye, the ratio of the two components was equal for all red dye formulations. The non-cross-linked gelatin matrices and the gelatin/formaldehyde matrices contained $0.0154 \pm 0.0005 \mu \mathrm{l} / \mathrm{mg}$ of sample and $0.0145 \pm$ $0.0003 \mu \mathrm{l} / \mathrm{mg}$ of sample, respectively. The gelatin/sucrose matrices contained $0.0128 \pm$ $0.0004 \mu \mathrm{l} / \mathrm{mg}$ of sample and the gelatin/fructose matrices contained $0.0130 \pm 0.0009$ $\mu \mathrm{l} / \mathrm{mg}$ of sample.

\section{Dye Studies - Qualitative Visual}

Visual studies revealed that the gelatin, gelatin/sugar, and gelatin/formaldehyde matrices gradually released dye over an extended period at ambient temperatures (Figure 43). Visual studies show that initial exposure to the solvent immediately leads to release, as seen in the concentrated dye color of the first time interval. Dye on the matrix surface or free dye within matrix pores may contribute to this. The aqueous solubility of the red and green dyes may also be responsible for the rapid release of the components from the matrices.

As seen in Figure 44, the release of the two components (blue and yellow) that make up the green color appear to occur at different rates. This would have significance in both the modeling of matrix release characteristics, as well as for formulation purposes (ability to deliver more than one therapeutic agent from the same matrix, but at differing 


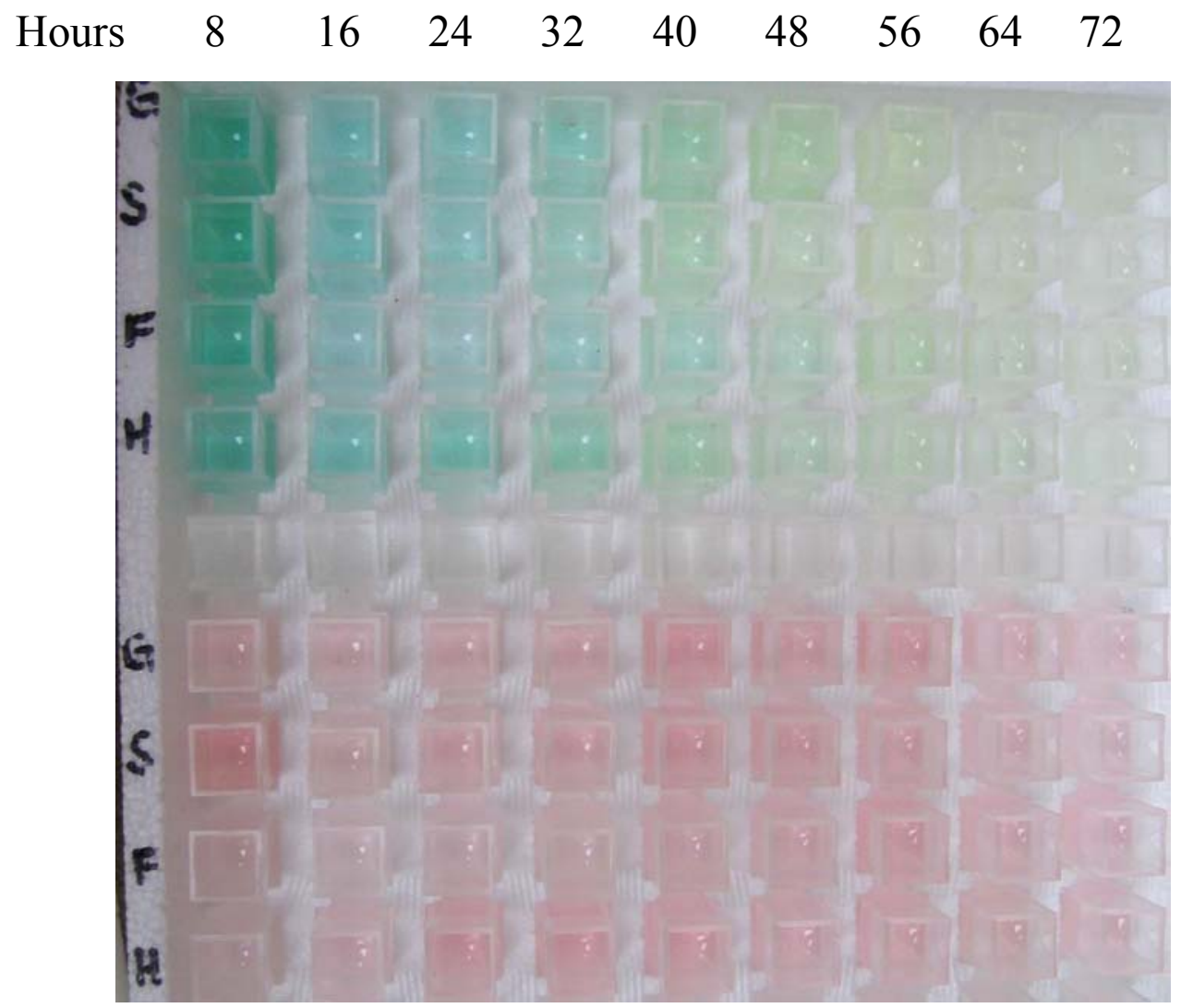

Figure 43. Overview of visual studies of gelatin (G), gelatin/sucrose (S), gelatin/fructose (F), gelatin/formaldehyde $(\mathrm{H})$ matrices loaded with green or red dye.

Matrices were refrigerated for 24 hours followed by 24 hours of lyophilization and evaluated under excess moisture and ambient temperatures. 


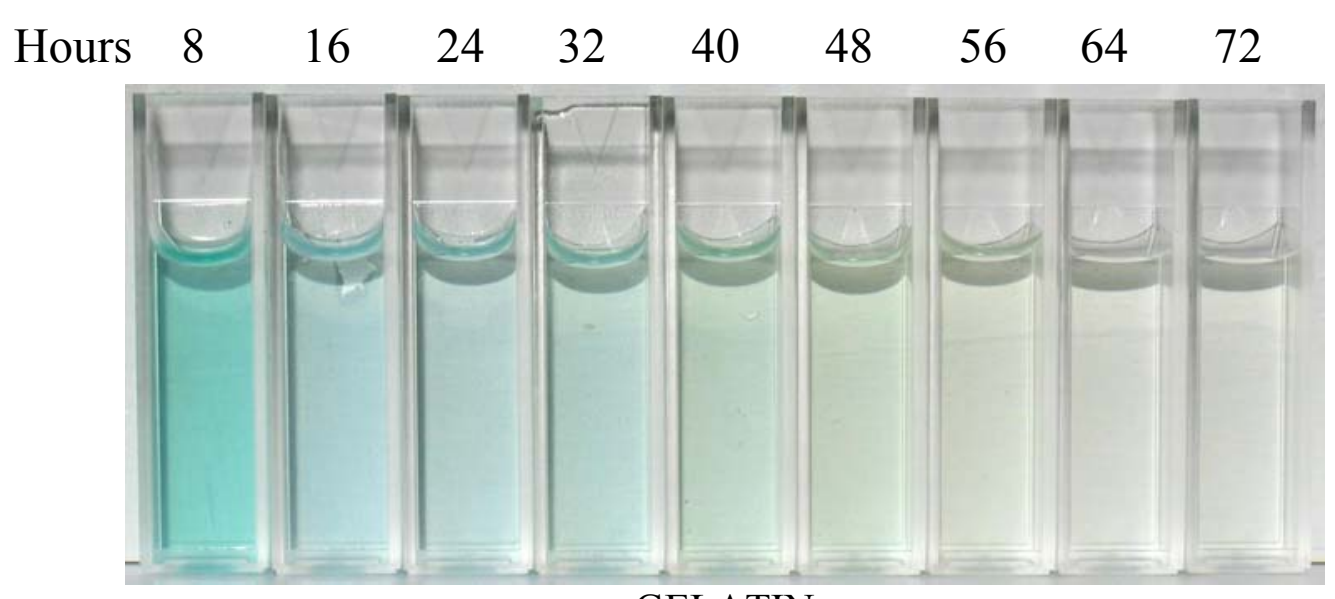

GELATIN

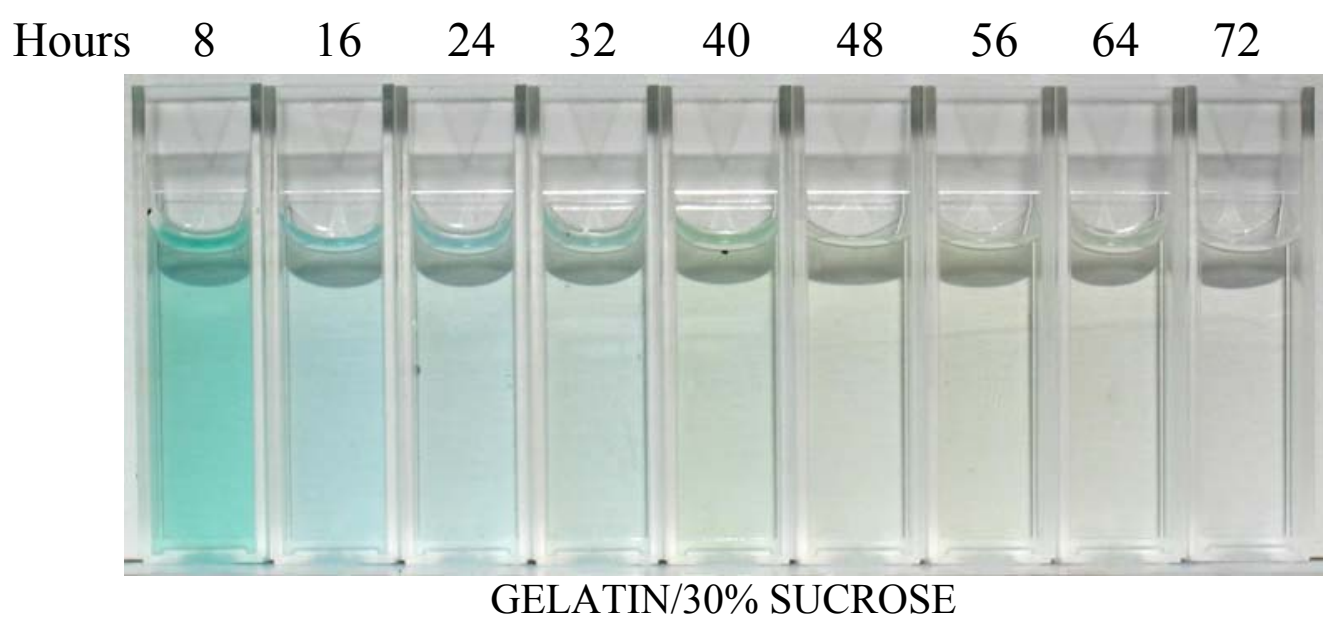

Figure 44. Visual studies of gelatin and gelatin/sucrose matrices loaded with $10 \%$ green dye.

Matrices were refrigerated for 24 hours followed by 24 hours of lyophilization and evaluated under excess moisture and ambient temperatures. 

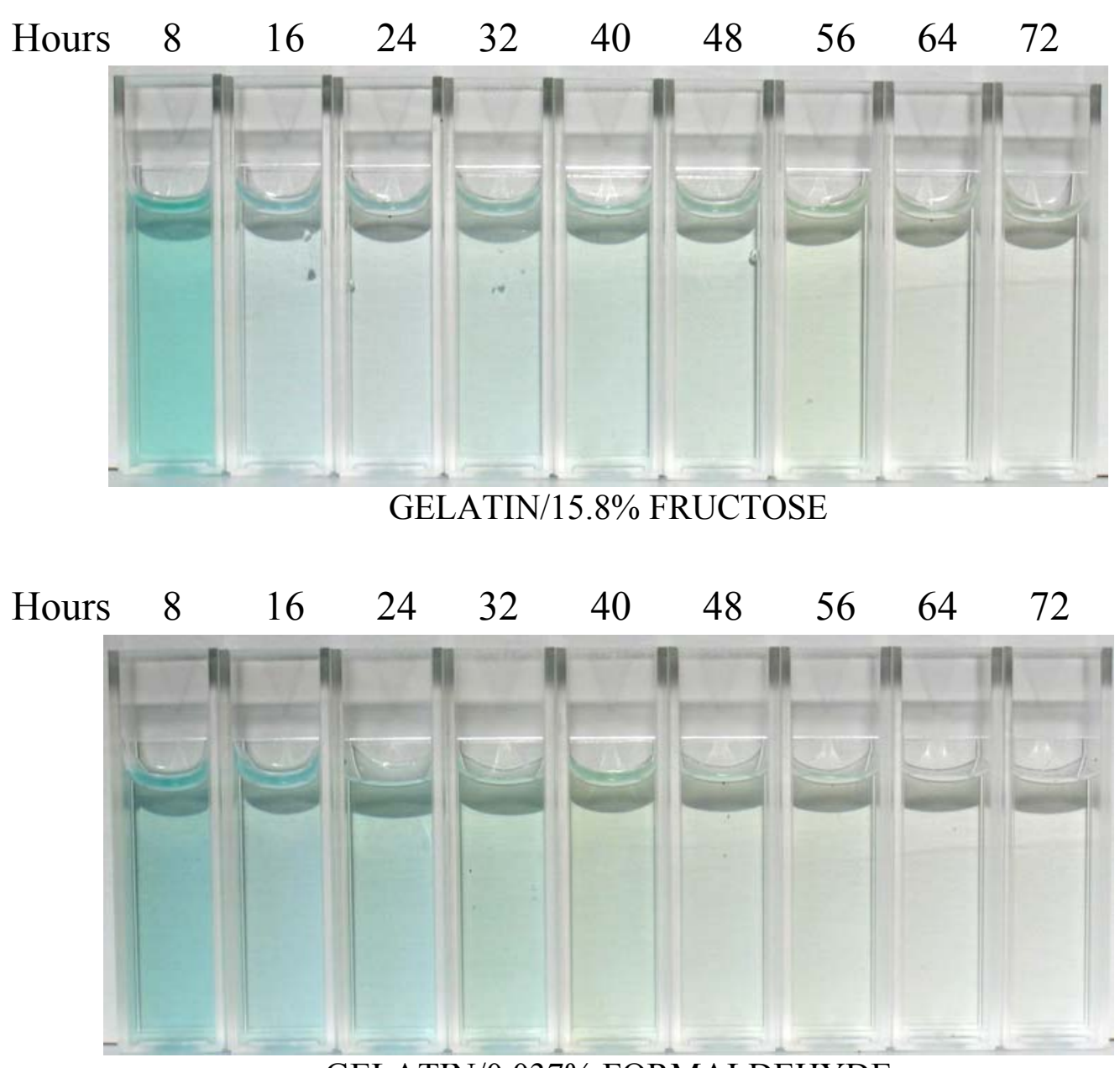

GELATIN/0.037\% FORMALDEHYDE

Figure 44 (continued). Visual studies of gelatin/fructose and gelatin/formaldehyde matrices loaded with $10 \%$ green dye.

Matrices were refrigerated for 24 hours followed by 24 hours of lyophilization and evaluated under excess moisture and ambient temperatures. 
rates). There appears to be a bluish shade followed by a light green shade of dye released until the amount of dye released is no longer visible. Each formulation demonstrated a different visual release period for the first shade of green of $48,40,48$, and 36 hours for non-cross-linked gelatin, gelatin/sucrose, gelatin/fructose and gelatin/formaldehyde matrices, respectively. After the initial concentrated release, the release of dye was gradual for 3 days and then diminished until no dye remained in the matrices.

The release of the red dye was different than the green loaded matrices (Figure 45). The red dye loaded matrices released a brief initial burst of dye followed by a lag time in which small amounts of dye were released over the first 8-12 hours. Around 1624 hours, a second burst of red is seen followed by a gradual release over the next 6 days. A slight separation of the two red dyes was seen, however, the separation was not as obvious as the green dye. The matrices maintained a tint of red in the matrix throughout the swelling and erosion process.

A study was performed in which both the red and green dyes were added together into the same gelatin matrix. The matrices loaded with red and green dye demonstrated a different visual profile than the matrices loaded with a single color. Visual studies show that the red and green dyes were released at different times and rates when in combination, with the green dye giving a quicker release from the matrix than the red dye (Figure 46). An initial burst of green dye from the matrix was seen in the first 4 hours. All four formulations demonstrated total visual release of green dye between 32 and 56 

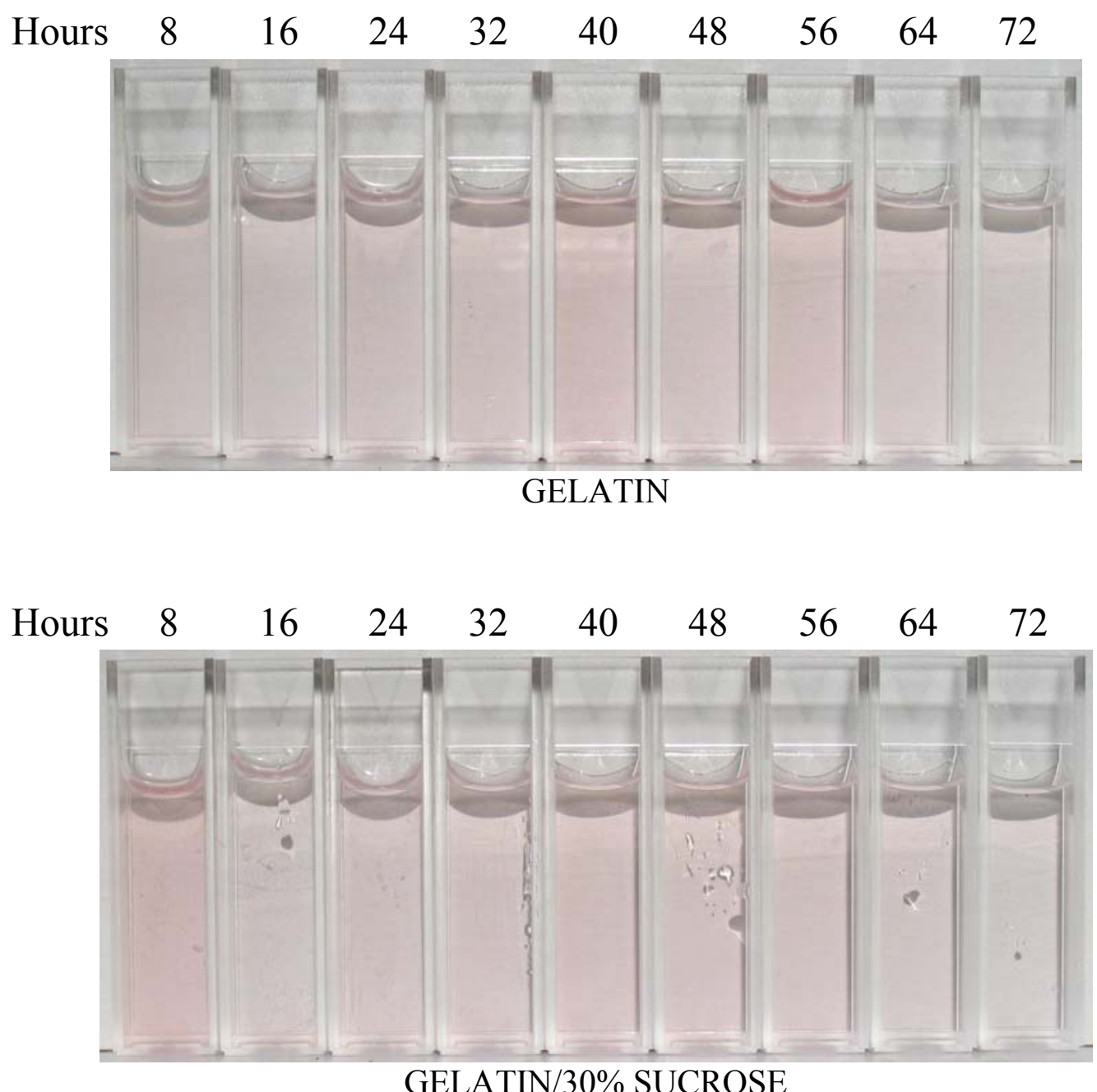

Figure 45. Visual studies of gelatin and gelatin/sucrose matrices loaded with $10 \%$ red dye.

Matrices were refrigerated for 24 hours followed by 24 hours of lyophilization and evaluated under excess moisture and ambient temperatures. 


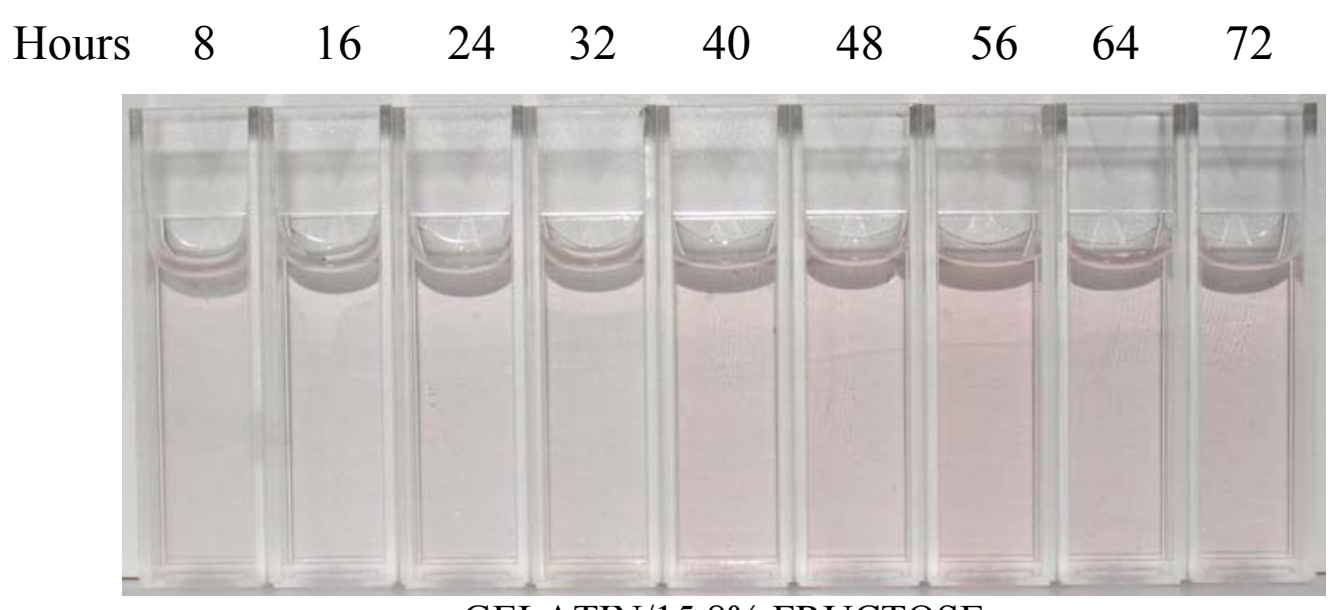

GELATIN/15.8\% FRUCTOSE

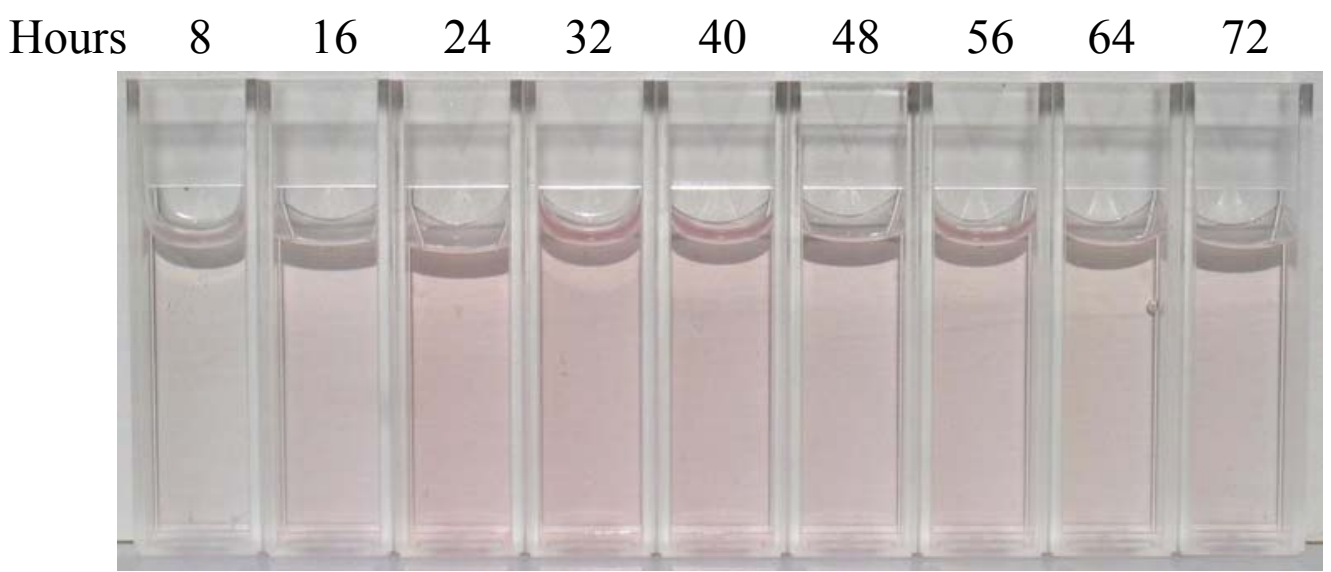

GELATIN/0.037\% FORMALDEHYDE

Figure 45 (continued). Visual studies of gelatin/fructose and gelatin/formaldehyde matrices loaded with $10 \%$ red dye.

Matrices were refrigerated for 24 hours followed by 24 hours of lyophilization and evaluated under excess moisture and ambient temperatures. 


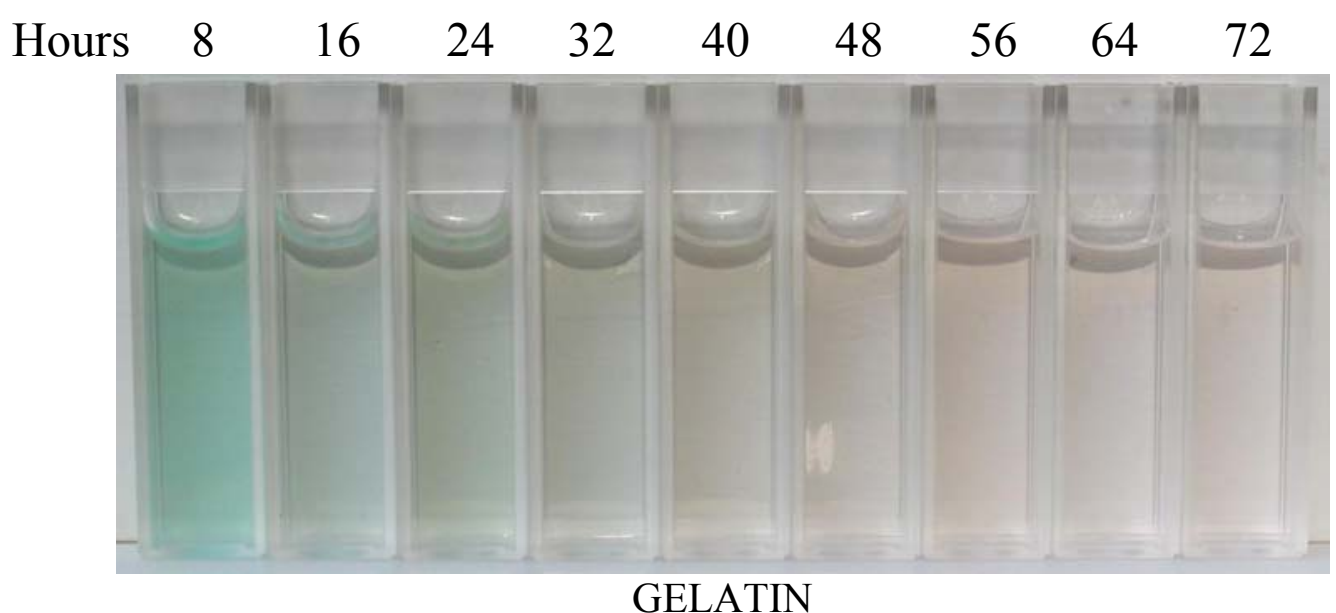

GELATIN

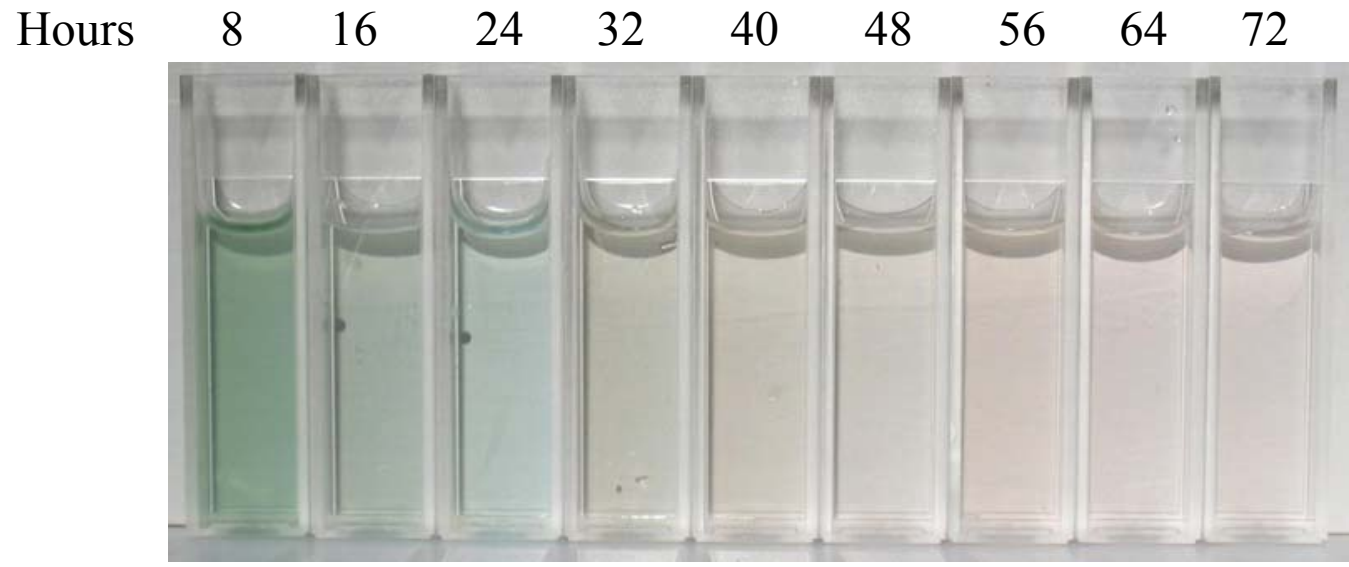

GELATIN/30\% SUCROSE

Figure 46. Visual studies of gelatin and gelatin/sucrose matrices loaded with $\mathbf{1 0 \%}$ green and $10 \%$ red dye.

Matrices were refrigerated for 24 hours followed by 24 hours of lyophilization and evaluated under excess moisture and ambient temperatures. 


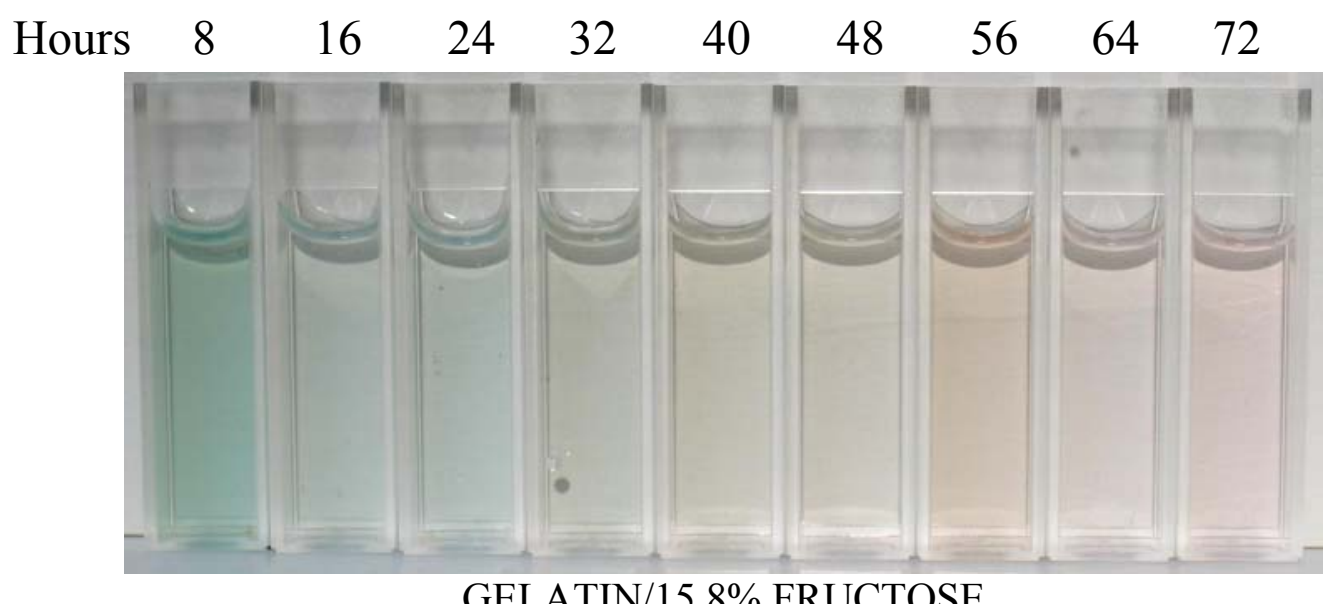

GELATIN/15.8\% FRUCTOSE

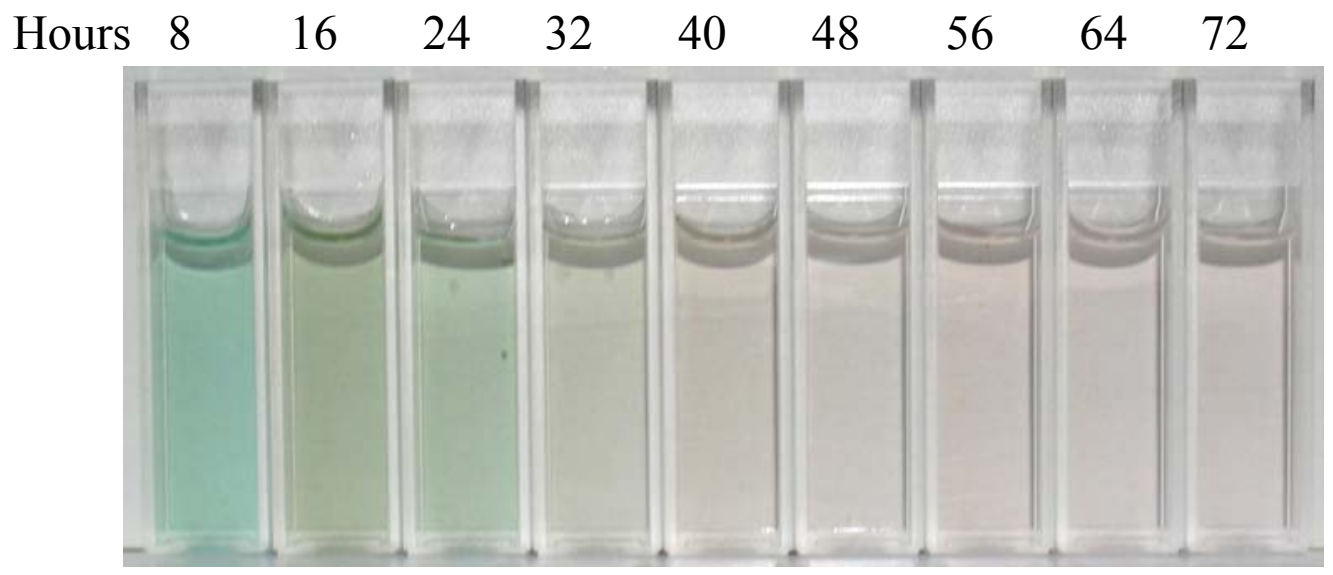

GELATIN/0.037\% FORMALDEHYDE

Figure 46 (continued). Visual studies of gelatin/fructose and gelatin/formaldehyde matrices loaded with $10 \%$ green and $10 \%$ red dye.

Matrices were refrigerated for 24 hours followed by 24 hours of lyophilization and evaluated under excess moisture and ambient temperatures. 
prominent around 72 hours followed by a continual release for an additional 10-15 days. The matrices maintained a tint of red in the matrix throughout the swelling and erosion process. The same separation of the blue and yellow components of the green dye was seen when the dyes were used in combination. However, the separation of the two red components was not as obvious when compared to the single color. Again, this data suggests solute release, to some extent, as a function of solute physicochemical properties, as well as the influence of one solute upon another's transport properties. The solute physicochemical properties that may affect release include surface area of the solid drug particle, drug solubility, the crystal or amorphous drug form, the salt form of the drug, net charge, the state of hydration, and molecular size. A structured crystalline drug is less soluble than an amorphous drug. The salt form of the drug directly affects the solubility of the drug. Sodium and potassium salts of weak acids and hydrochloride salts of weak bases are more soluble than their parent compounds.

At ambient temperatures, all four matrices remained intact until the film reached an aqueous saturation point, at which time the matrices became maximally swollen, resulting in the disintegration of the matrix into small pieces. The gelatin, gelatin/sucrose, and gelatin/fructose matrices remained intact for 20-24 days while the gelatin/formaldehyde matrices lasted the longest (30-35 days). At Day 28, as the gelatin/formaldehyde matrices eroded away, small pieces of cross-linked gelatin and formaldehyde tendrils were visible in the solution. The parts of the matrix that were not covalently linked likely eroded away faster than the covalently linked ones. 


\section{Dye Studies - Quantitative Release}

The visual dye release studies allowed for the qualitative analysis of the matrices' drug release characteristics, so the dyes were also utilized in the in vitro quantitative release studies. In order to mimic the conditions of the disease states in which this delivery system could be used in therapy, limited volumes and varying turnover rates were utilized to evaluate dye release. An assessment of maintenance of sink conditions versus more concentrated conditions was also critical to data interpretation.

\section{A. Non-sink Conditions}

In vitro dye release studies agreed with the visual release studies revealing that the first 12 hours of release are the most characteristic for the release profile. The release profiles show that the rate of release changes across time. In general, the dye is released at a quicker rate for the first twelve hours, at which time the rate decreases over the next 60 hours. This may be due the saturation of the dissolution medium, or more likely due to a change in the concentration gradient. The study was designed for a physiological area with limited volume and slow fluid turnover rate, so the medium was not changed during the study. As the study progressed, the concentration gradient became smaller as dye was released out of the matrix into the medium. This may have caused a significant change in the release rates.

The amount and rate of dye released from non-cross-linked gelatin matrices show a quick release of dye within the first 8-16 hours of the study. After 72 hours, the red samples had released $30 \%$ of the dye that was loaded in the samples (Figure 47). After 6 days (data not shown), $45-50 \%$ of the dye was still in the sample. Figure 47 shows that the green dye samples released $55-60 \%$ of the dye within the first 72 hours of the study. 


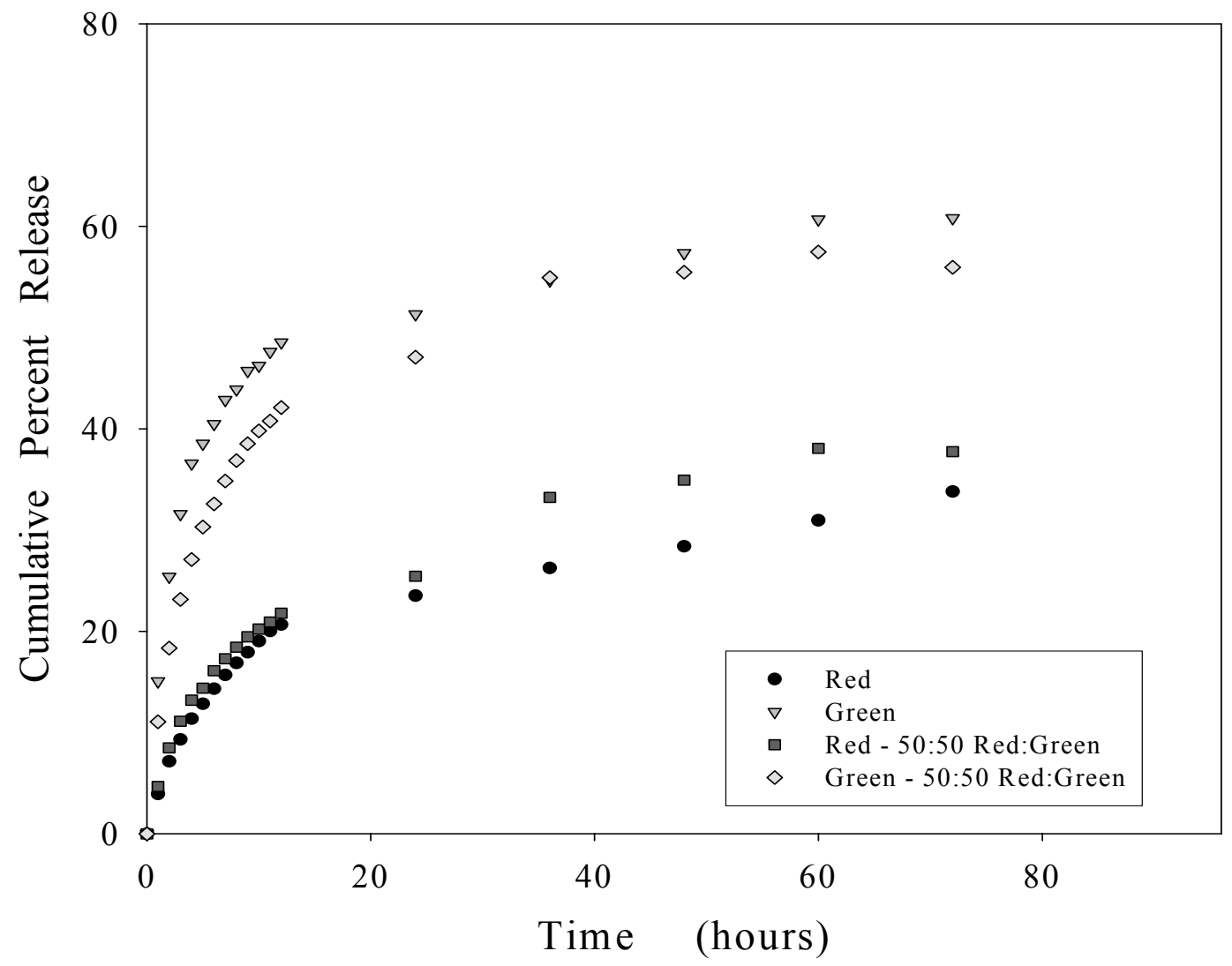

Figure 47. Release profile of non-cross-linked gelatin matrices loaded with red, green, or a combination of red \& green dye.

Matrices were refrigerated for 24 hours followed by 24 hours of lyophilization and evaluated under excess moisture and ambient temperatures. The dissolution medium was not changed throughout the study. The results are plotted as an average of triplicate measurements. 
The in vitro studies also show little difference between the single dye or combination dye formulations' release kinetics. This may be due to the dyes' solubility, allowing the dyes to freely diffuse out of the matrix, along with what appears to be a lack of interference with the matrix or between the dye as the dyes diffuse out.

The amount and rate of dye released from gelatin/sucrose matrices show a quick release in the first 8 hours (Figure 48). After 72 hours, the matrices had released $40 \%$ of the loaded red dye and $60 \%$ of the green dye that was loaded into the matrices. No difference was seen in the release of a single dye versus the same dye in combination of the two dyes. Of note is that, the dye release profiles for the gelatin/sucrose and the noncross-linked gelatin formulations are quite similar.

The release of red dye from gelatin/fructose matrices is somewhat slower than from the gelatin/sucrose matrices (Figure 49). After 72 hours, the red samples released $35-40 \%$ of the red dye within the matrices. Seventy percent of the green dye loaded in the matrices was released from the gelatin/fructose matrices within the first 72 hours, with a somewhat slower initial release. The release profile of the matrices with a combination of red and green dye show a more pronounced effect on the individual dyes' release than was observed with gelatin/sucrose or non-cross-linked gelatin matrices. Notably, a lower percent of green dye was released after 72 hours, an approximate $20 \%$ decrease. Since the red dyes' release was minimally affected when in combination, it is possible that an interaction between the two solutes may be minimized in the gelatin/sucrose matrix, but in the presence of any excess red dye or fructose in the 


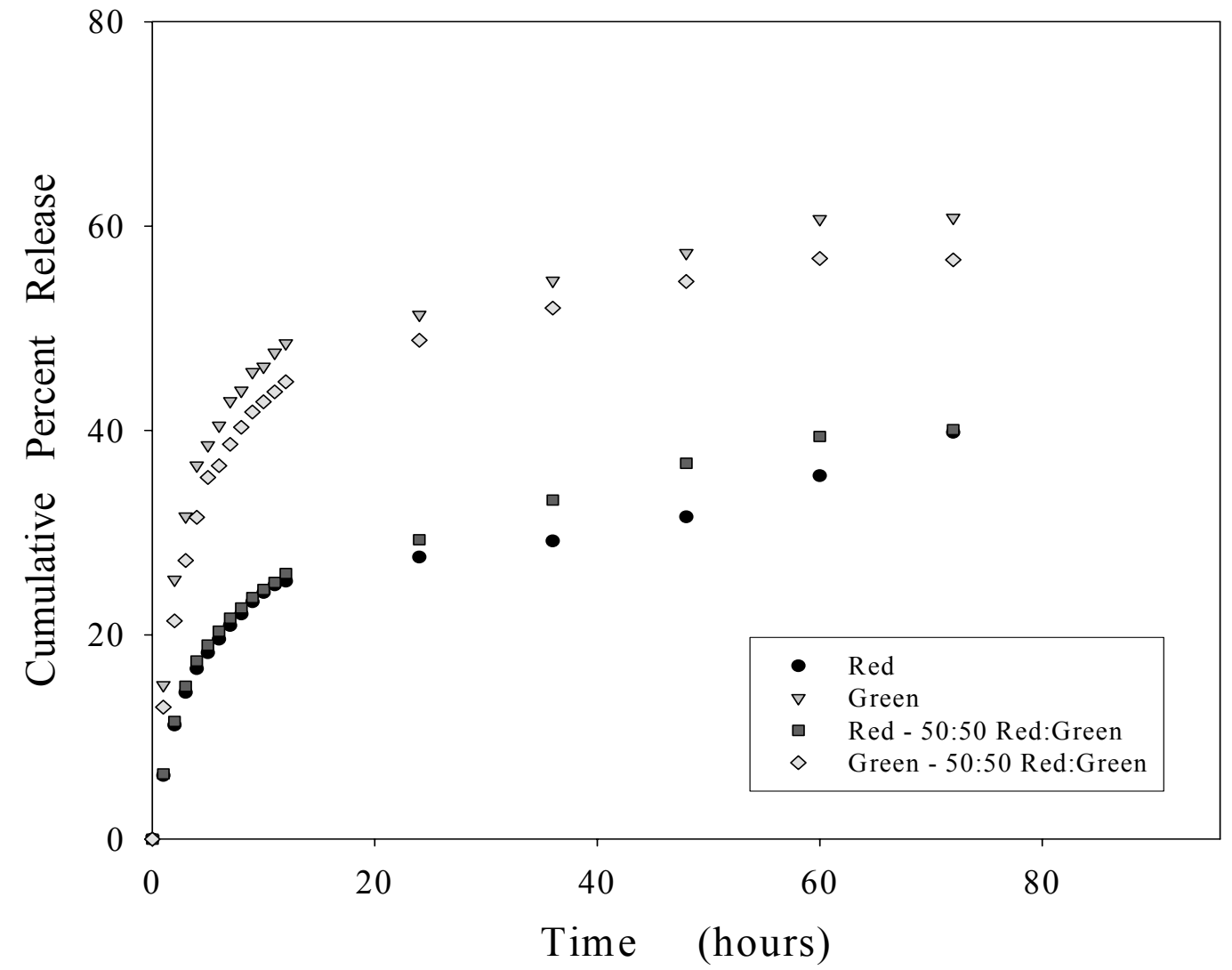

Figure 48. Release profile of gelatin/30\% sucrose matrices loaded with red, green, or a combination of red \& green dye.

Matrices were refrigerated for 24 hours followed by 24 hours of lyophilization and evaluated under excess moisture and ambient temperatures. The dissolution medium was not changed throughout the study. The results are plotted as an average of triplicate measurements. 


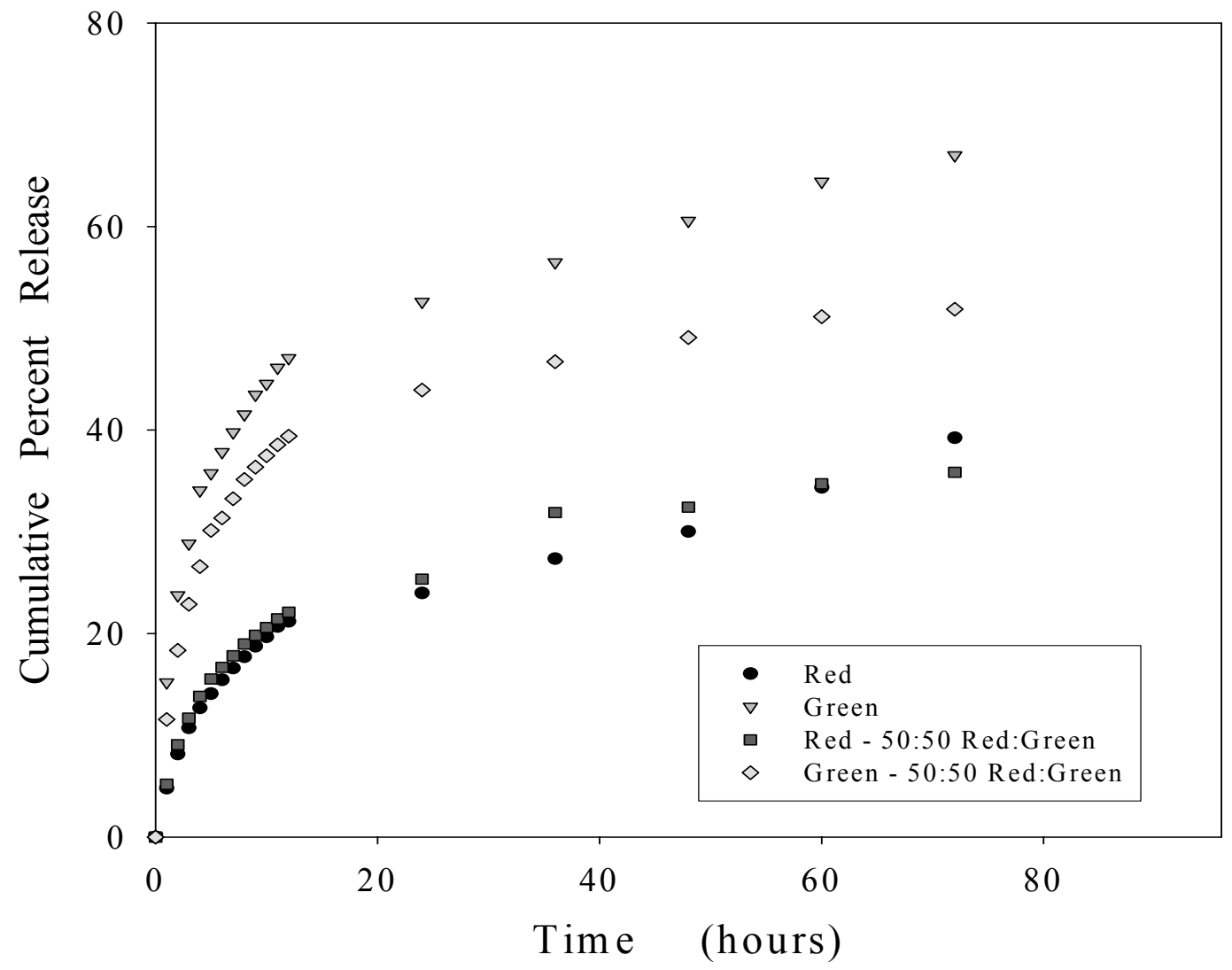

Figure 49. Release profile of gelatin/15.8\% fructose matrices loaded with red, green, or a combination of red \& green dye.

Matrices were refrigerated for 24 hours followed by 24 hours of lyophilization and evaluated under excess moisture and ambient temperatures. The dissolution medium was not changed throughout the study. The results are plotted as an average of triplicate measurements. 
gelatin/fructose matrix, the release of the green dye slows down. It is of note that the red dye is comprised of larger bulkier molecules than the green dye.

The amount and rate of dye released from gelatin/formaldehyde matrices demonstrated different release rates when compared to the other matrices (Figure 50). The release of red dye did not show an initial burst of dye but a slow steady release for the first 12 hours followed by an even slower release. At 72 hours, the red samples released $20 \%$ of the dye loaded in the sample. The green loaded matrices show the same decrease in dye released ( $40 \%$ compared to $55-65 \%$ in the other matrices). When the two dye colors were evaluated in combination, the release of both dyes increased significantly. If the formaldehyde matrices are more tightly structured than the other matrices, this may be due to the excess free dye present in the matrix, forcing the dyes to diffuse out quickly. Also, if the dyes have a slight charge attraction to sites on the gelatin molecule, this could slow release. However, when the amount of dye added is doubled (two colors), these sites may become saturated, leaving excess dye available to diffuse out of the system.

Overall, the green dye releases at a faster rate and to a significantly larger extent than the red dye. This may be due to a different concentration gradient for the red dye in the sample when compared with the green dye. The concentration of the dyes is unknown due to the use of a pre-formulated dye from McCormick \& Co, Inc (Hunt Valley, MD). It is possible that a smaller weight quantity of red dye may be needed to produce a brightness equivalent to the green dye. The ratio of the two red dyes may also 


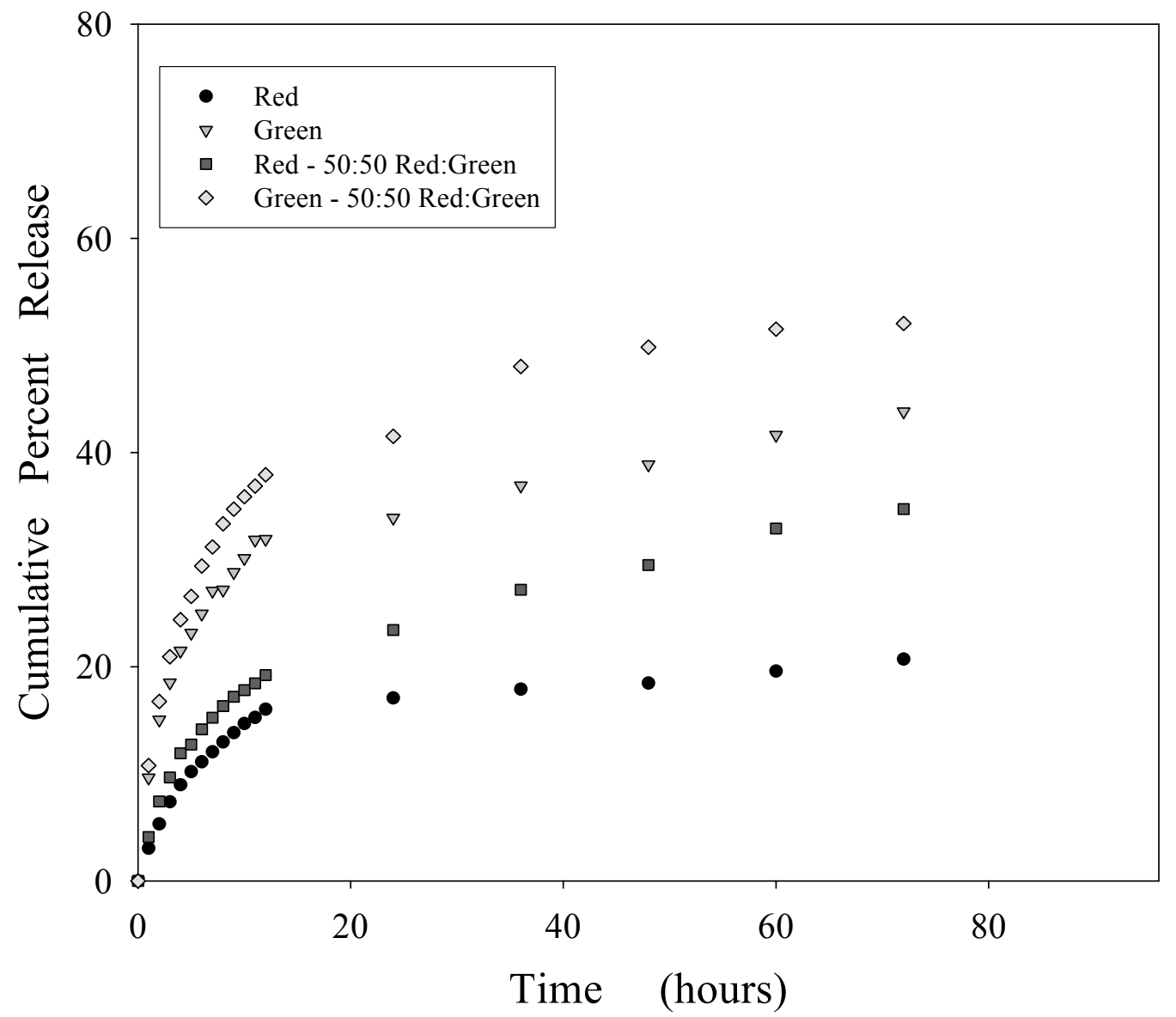

Figure 50. Release profile of gelatin/ $\mathbf{0 . 0 3 7 \%}$ formaldehyde matrices loaded with red, green, or a combination of red \& green dye.

Matrices were refrigerated for 24 hours followed by 24 hours of lyophilization and evaluated under excess moisture and ambient temperatures. The dissolution medium was not changed throughout the study. The results are plotted as an average of triplicate measurements. 
be different than the ratio of blue and yellow components used in the green dye. Also, since the concentration of the dyes is not known, then an absolute estimation of sink conditions (in the experiment) cannot be determined. Based on dye chemistries, it is likely that the red dye would have a lower solubility than the green; this too would influence the driving force for dye release. A second reason for the difference may be that the red dye becomes entangled with the gelatin or cross-linking agents to a greater extent than the blue or yellow components of the dye. This may be due to the bulkiness of the one red component, erythrosine. Thus, red dye diffusion may be more limited. The erythrosine has 4 -iodo groups attached to it which can become entangled within the matrix. As the gelatin strands become mobile, the green dye is able to become free and diffuse out of the matrix. The iodine loaded dye, however, may require more time and a larger number of free strands before it is mobile enough to diffuse through the channels and out of the matrix. Also, a bulkier component may require a longer pathway to get from its original position within the matrix into the solvent. As the erythrosine moves along its path, it may get entangled with another strand or blocked by a new disentangling strand in which a smaller molecule may be better able to diffuse around the strand. The weight of the diffusing molecules may also slow down the diffusion of the red components.

After 6 days of release, only $60-70 \%$ of the green dye and $20-40 \%$ of the red dye have been accounted for during dye release. The remaining dye that has not been accounted for may be due to the loss of dye when the samples were blotted dry during the weighing portion of the swelling/bioerosion section of the studies. A second reason for 
such a difference may be due to dye levels in the sample that were lower than the level of detection. At the end of the 6 days, the change in the concentration in the medium may have been so small that the lamp was unable to detect a change. A third reason for unaccountable dye may be due to the retention of the dye through a direct chemical interaction with the gelatin or cross-linking agent. Finally, unaccountable dye may due to the retention of the dye in the matrix. At the six day time interval, there was a significant amount of red dye (40-60\%) still remaining in the matrix, with the green dye retaining 10 to $20 \%$ of the dye. Upon visual analysis of the matrices on day 6 , a slight tint of green was visible within the matrices loaded with green dye. One the other hand, the red loaded matrices demonstrated a bright ring of dye located at the center of all the matrices. It is possible that the transport of the red dye is slowed/stopped due to the loss of its concentration gradient.

A comparison of swelling/erosion and dye release was completed to assess whether diffusion is the major driving force for release. Figures 51-54 show that, upon exposure to the water, the matrices take up a substantial quantity of water within the first 16 hours. The matrices then maintain a constant weight throughout the 6 day study. This indicates that there are two dynamic processes, swelling and erosion taking place during the course of the release profile. The first process is swelling in which the matrices take up water and begin to swell. Eventually, the matrices reach static condition, in which there is either an equilibrium between swelling and erosion or the matrix ceases to swell (reaches maximum water content) but is not eroding, as the dye continues to be released. Finally, the second process, erosion, causes the gelatin strands 


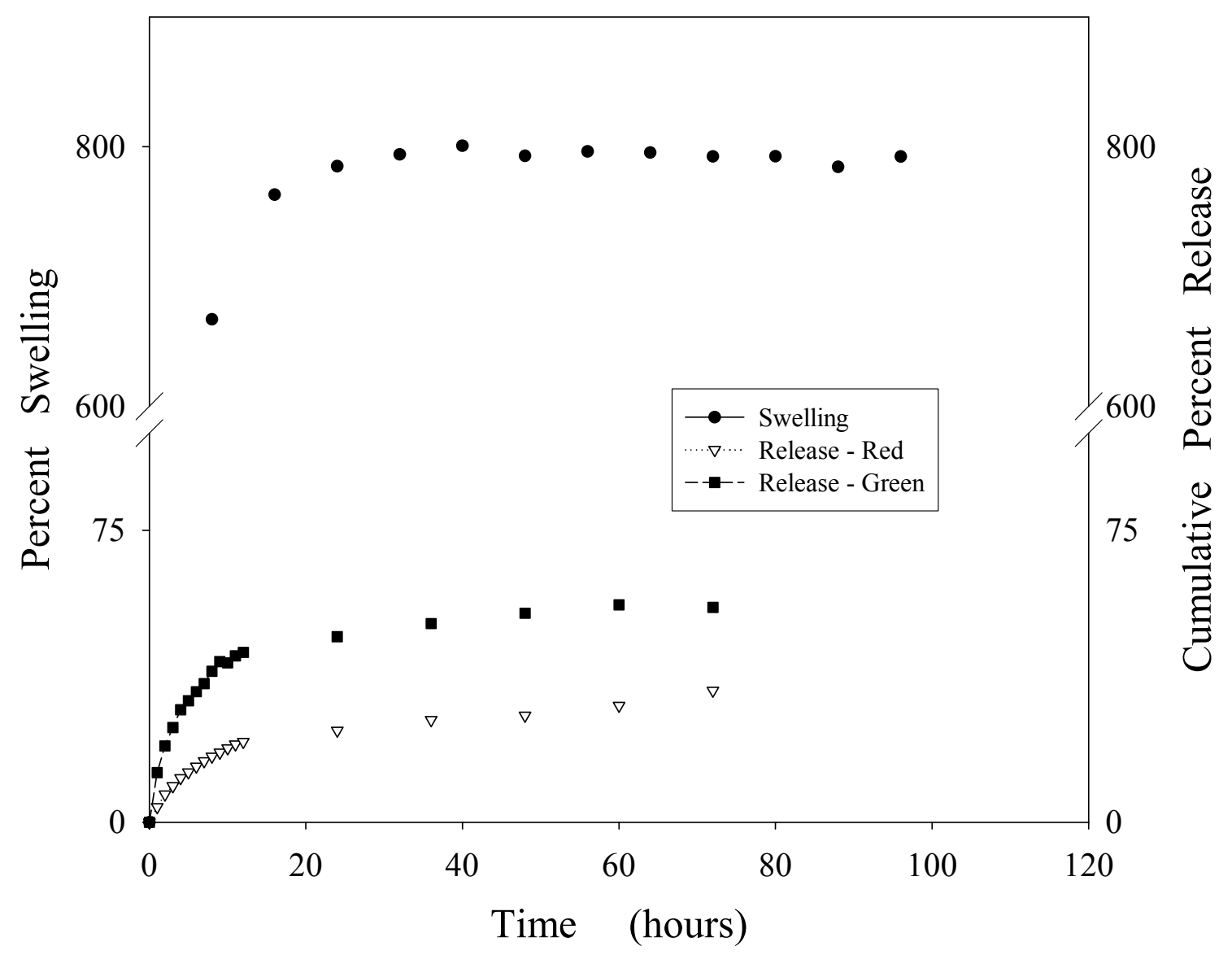

Figure 51. Comparison of the release profile to the swelling/erosion profile of gelatin matrices.

Matrices were refrigerated for 24 hours followed by 24 hours of lyophilization and evaluated under excess moisture and ambient temperatures. The dissolution medium was not changed throughout the study. The results are plotted as an average of triplicate measurements. 


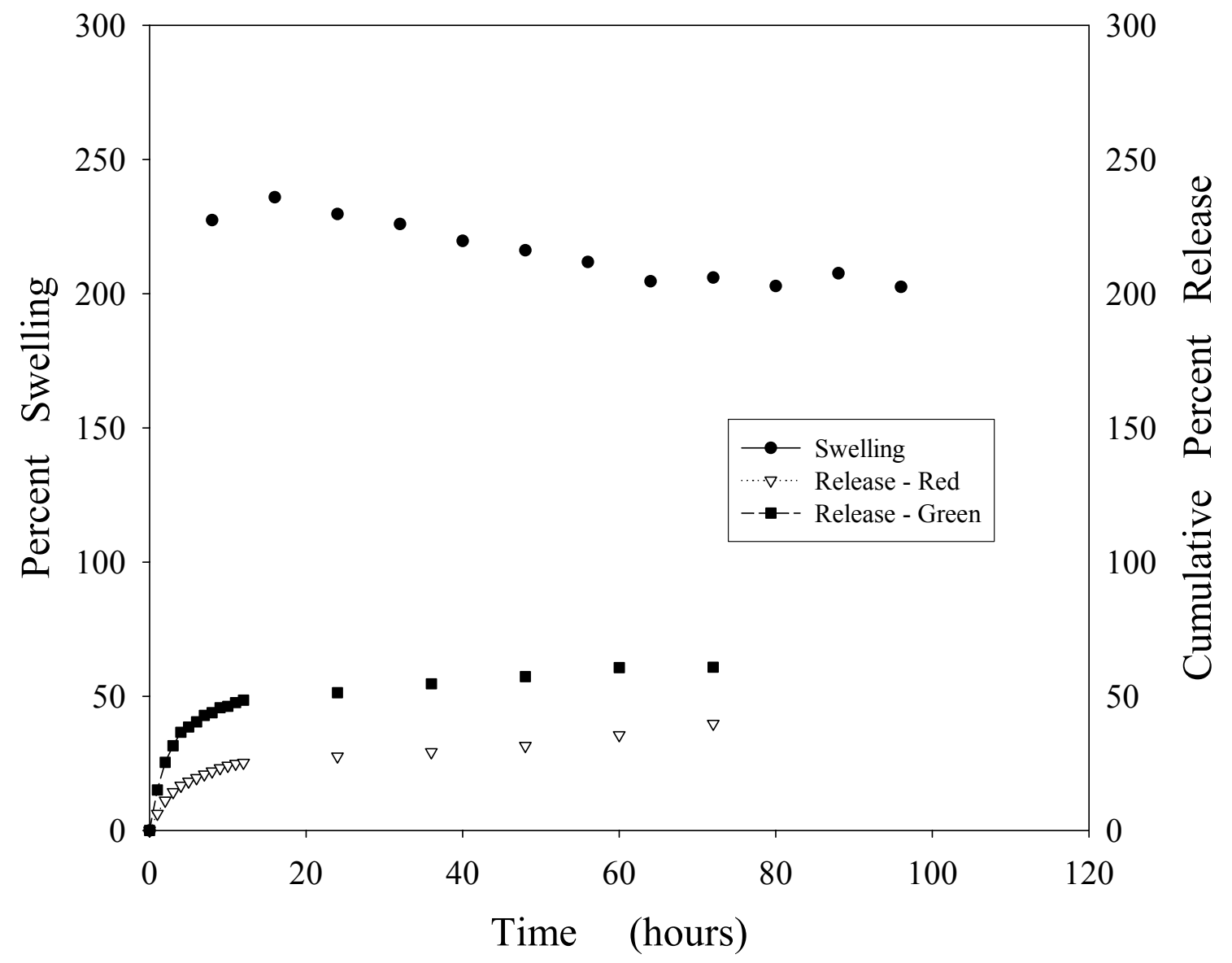

Figure 52. Comparison of the release profile to the swelling/erosion profile of gelatin $/ \mathbf{3 0} \%$ sucrose matrices.

Matrices were refrigerated for 24 hours followed by 24 hours of lyophilization and evaluated under excess moisture and ambient temperatures. The dissolution medium was not changed throughout the study. The results are plotted as an average of triplicate measurements. 


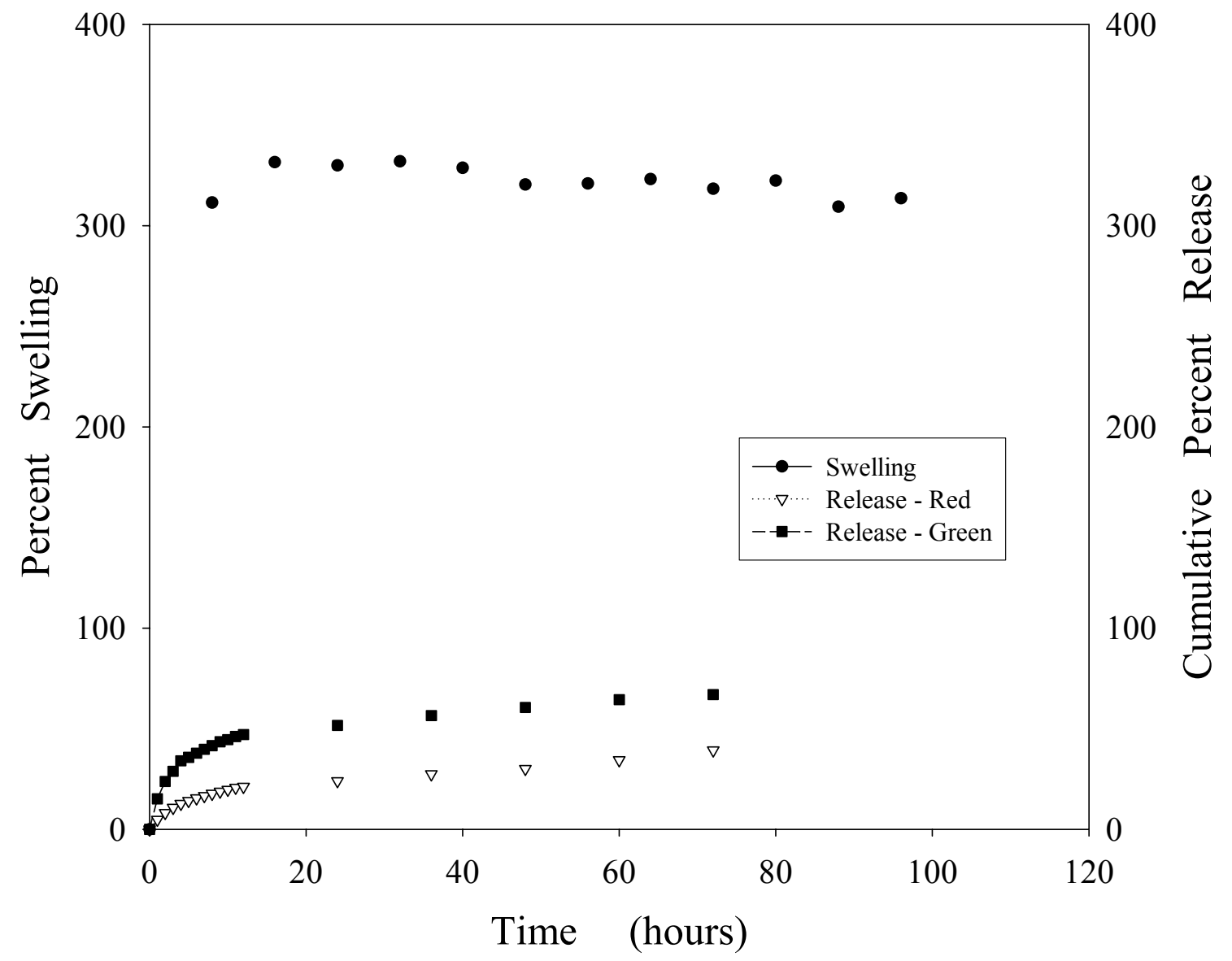

Figure 53. Comparison of the release profile to the swelling/erosion profile of gelatin/15.8\% fructose matrices.

Matrices were refrigerated for 24 hours followed by 24 hours of lyophilization and evaluated under excess moisture and ambient temperatures. The dissolution medium was not changed throughout the study. The results are plotted as an average of triplicate measurements. 


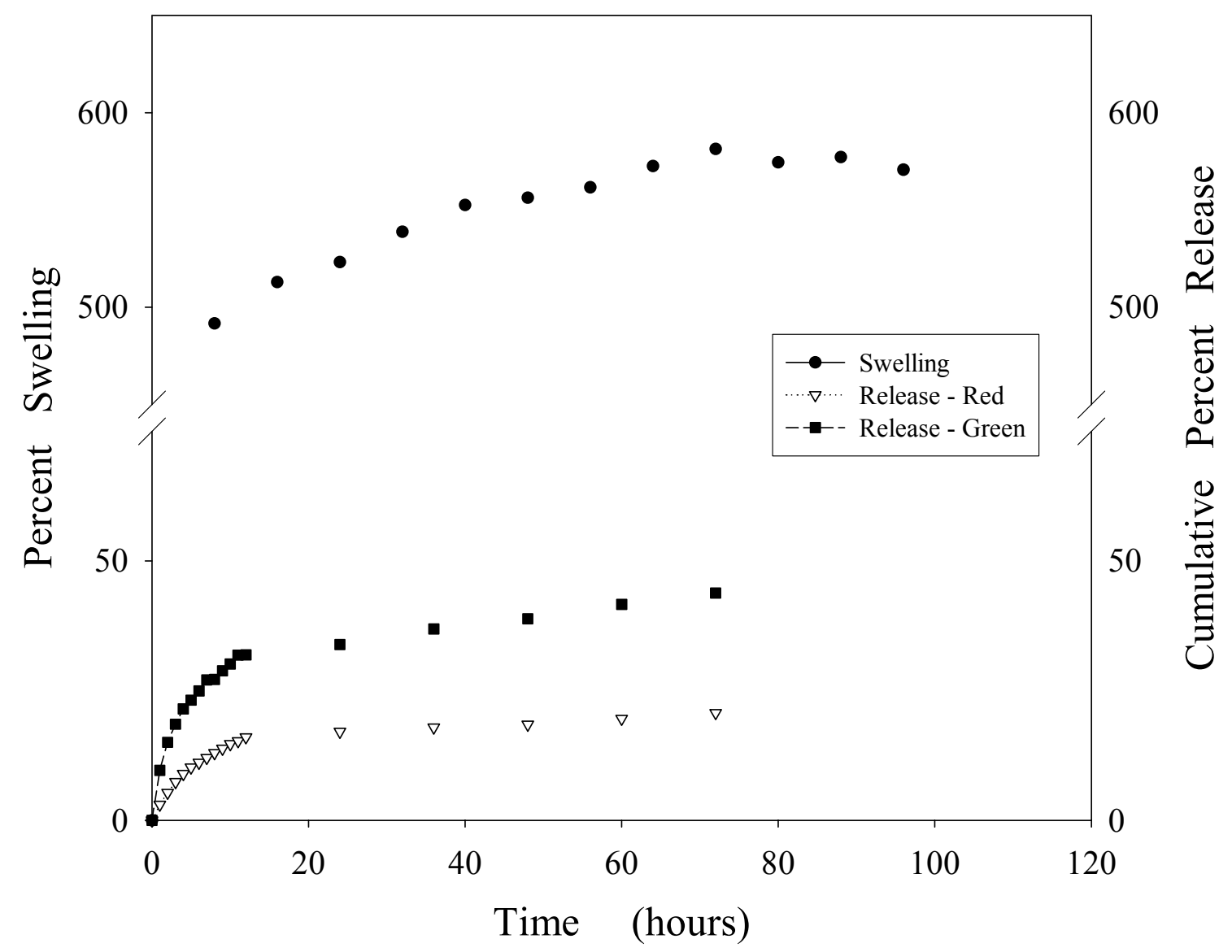

Figure 54. Comparison of the release profile to the swelling/erosion profile of gelatin/0.037\% formaldehyde matrices.

Matrices were refrigerated for 24 hours followed by 24 hours of lyophilization and evaluated under excess moisture and ambient temperatures. The dissolution medium was not changed throughout the study. The results are plotted as an average of triplicate measurements. 
to disentangle, bonds to break, and the matrices to fall apart. In addition, the rate of dye release and the swelling rate are different indicating that the two parameters are different. All four formulations show a slow continual release throughout the static phase indicating that diffusion is the driving force. This confirms the diffusion-controlled process because a swelling controlled soluble matrix swells, reaches a swelling-erosion equilibrium and demonstrates rapid zero order release [7].

\section{B. Sink Conditions}

In vitro release studies under sink conditions agreed with the visual release studies revealing that the first 2 days of release are the for the release profile. The release profiles show that the rate of release changes across time. In general, the release of dye is quicker during the first 32 hours, at which time the rate decreases over the next 30 hours. This may be due a slight change of the concentration gradient. The amount of dye loaded in the matrices is small so a slight change in the gradient may appear to have a large affect on the release.

The amount of dye released from the non-cross-linked gelatin matrices show a quick release of dye within the first 12-16 hours of the study for the green dye and the

first 20-24 hours for the red dye. After 72 hours, the red samples had released $40 \%$ of the dye that was loaded in the samples (Figure 55). Also shown in Figure 55, the green dye samples released $60-65 \%$ of the dye within the first 72 hours of the study. The in vitro release studies show slight differences in the release profiles between the single dye and the combination dye formulations. The combination dye matrices appear to release the 


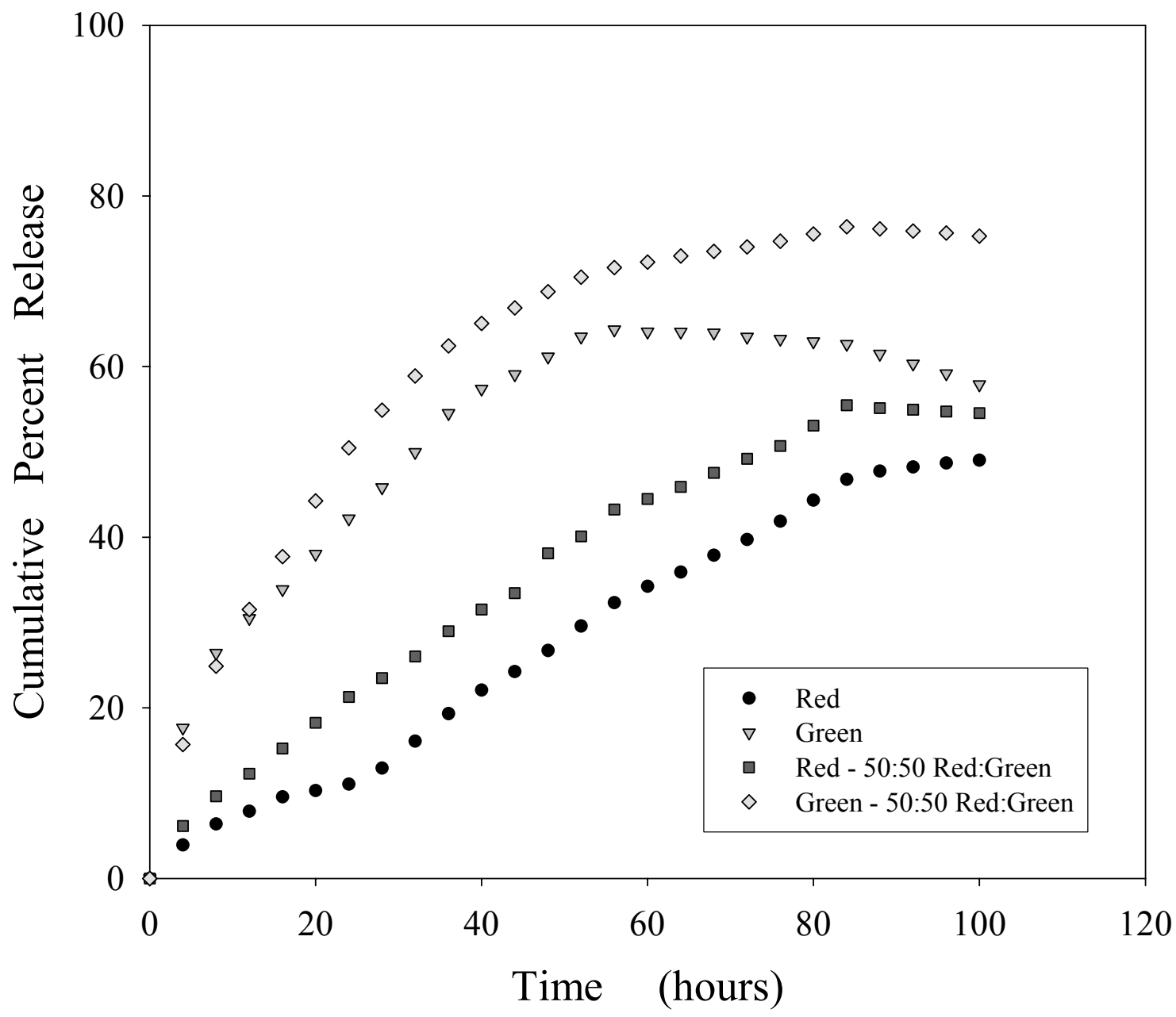

Figure 55. Release profile of non-cross-linked gelatin matrices loaded with red, green, or a combination of red \& green dye.

Matrices were refrigerated for 24 hours followed by 24 hours of lyophilization and evaluated under excess moisture, ambient temperatures, and sink conditions. The results are plotted as an average of triplicate measurements. 
dye at a faster rate. When in combination, the red and green dyes may be competing for the same binding or charge sites on the gelatin resulting in more free red dye. The excess red dye can then diffuse out of the matrix faster than the bound dye in the single matrix.

The release profile of the gelatin/sucrose matrices show a quick release of green dye in the first 12-16 hours (Figure 56). After 72 hours, the matrices released $45 \%$ of the red dye and $65-70 \%$ of the green dye that was loaded into the matrices. Slight differences were seen in the release of a single dye versus the same dye in combination of the two dyes. For the green loaded matrices, small differences were seen for the first 12-16 hours, at which time the two profiles separated with the combination matrices releasing 20 to $25 \%$ more green dye than the single dye matrices. The same separation of the release profiles is seen with the red loaded matrices, however, the separation of the profiles occurs within the first several hours of the study. As with the non-cross-linked gelatin matrices, the combination of the two dyes may lead to an excess of free dye within the matrix that can diffuse out of the matrix at a faster rate than the single dye matrix.

The amount and rate of dye released from the gelatin/fructose matrices show a quick release within the first 8-12 hours (Figure 57). After 72 hours, the red samples released $40 \%$ of the loaded red dye and $70 \%$ of the green dye that was loaded into the matrices. No difference was seen in the release of a single dye versus the same dye in combination of the two dyes. This may be due to additional interactions of the dye with 


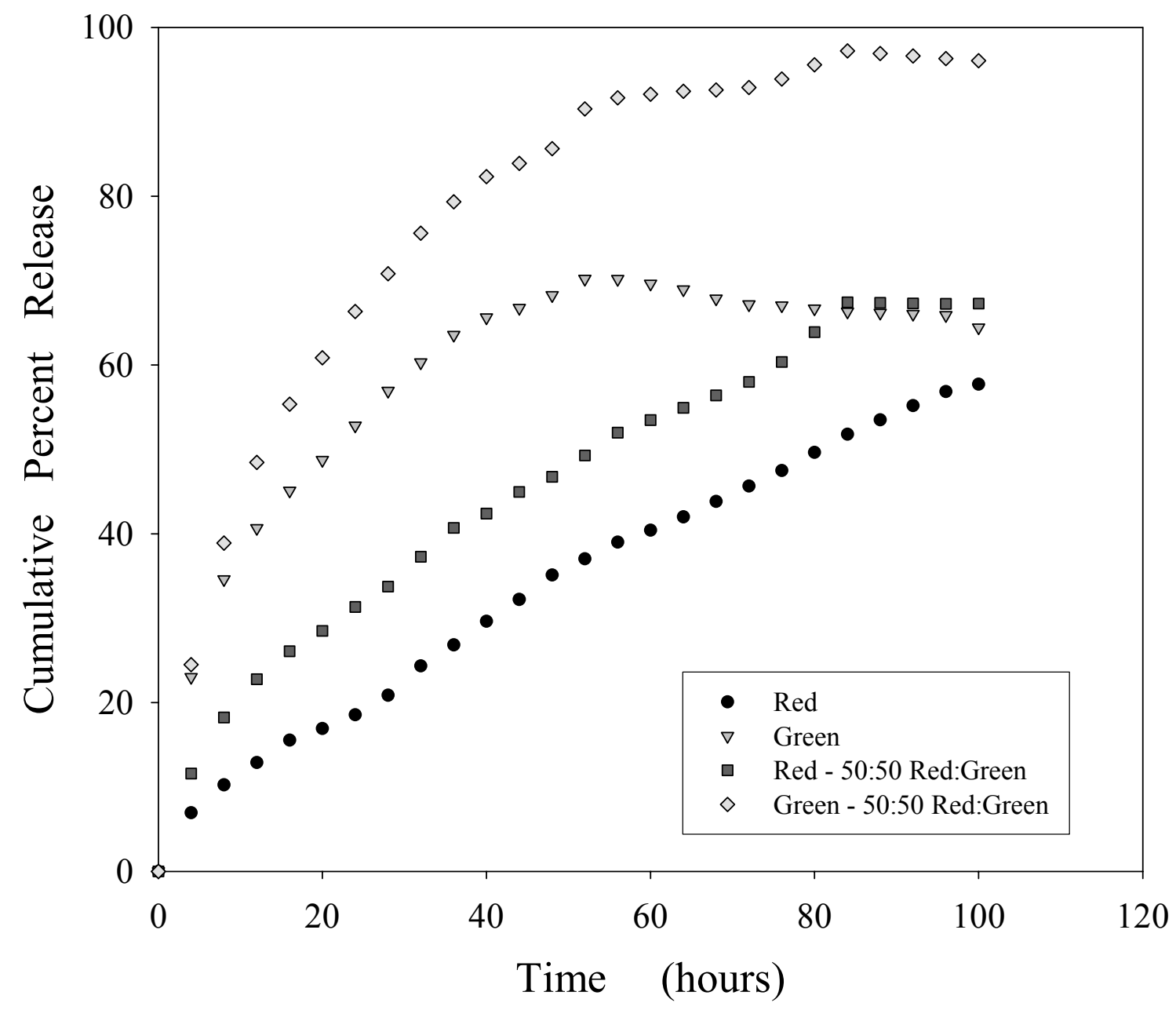

Figure 56. Release profile of non-cross-linked gelatin/30\% sucrose matrices loaded with red, green, or a combination of red $\&$ green dye.

Matrices were refrigerated for 24 hours followed by 24 hours of lyophilization and evaluated under excess moisture, ambient temperatures, and sink conditions. The results are plotted as an average of triplicate measurements. 


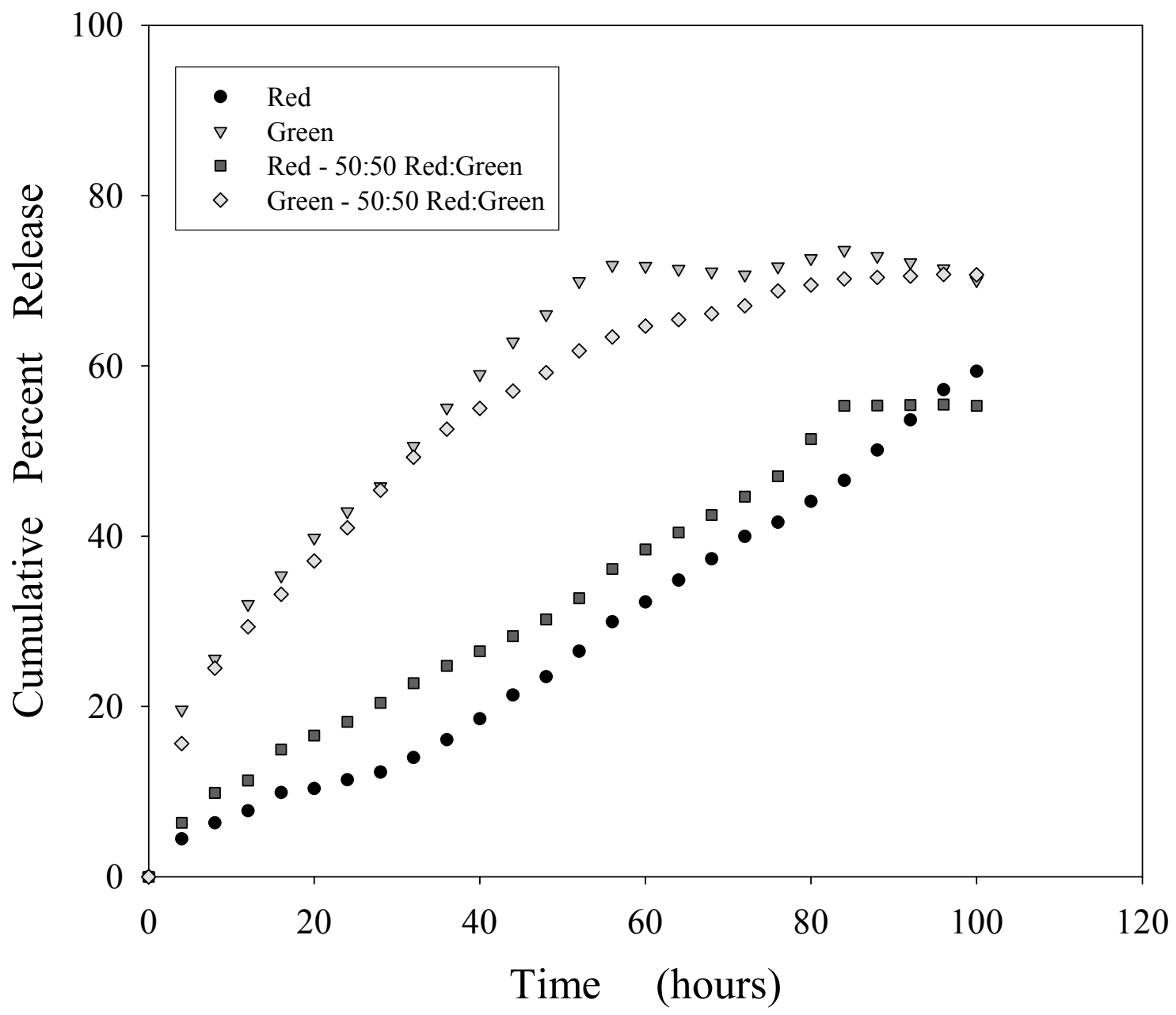

Figure 57. Release profile of non-cross-linked gelatin/15.8\% fructose matrices loaded with red, green, or a combination of red \& green dye.

Matrices were refrigerated for 24 hours followed by 24 hours of lyophilization and evaluated under excess moisture, ambient temperatures, and sink conditions. The results are plotted as an average of triplicate measurements. 
the fructose. Of the two sugars, fructose is more likely than sucrose to be in the reactive ring open form allowing a greater opportunity for the dye to interact with the fructose resulting in a smaller quantity of free dye.

The amount and rate of dye released from the gelatin/formaldehyde matrices demonstrated similar release profiles when compared to the other matrices (Figure 58). The release profiles of the dye show an initial release of dye over the first 24 hours of the study. At 72 hours, the matrices released $50-55 \%$ of the loaded red dye and $55-60 \%$ of the green dye that was loaded in the matrices. When the two dyes were evaluated in combination, the release of both dyes increased significantly. Due to the low concentration of formaldehyde, the gelatin/formaldehyde matrices demonstrate similar profiles as the non-cross-linked gelatin matrices. If the formaldehyde matrices are more tightly structured than the other matrices, this may be due to the excess free dye present in the matrix, forcing the dye to diffuse out quickly. Also, if the dyes have a slight charge attraction to sites on the gelatin molecule, this could slow release. However, when the amount of dye added is doubled (two colors), these sites may become saturated, leaving excess dye available to diffuse out of the system.

As seen with non-sink conditions, the green dye releases at a faster rate and to a significantly larger extent than the red dye. This may be due to a different concentration gradient for the red dye in the sample compared to the green dye. The concentrations of the dyes is unknown due to the use of a pre-formulated dye solution from McCormick \& Co, Inc (Hunt Valley, MD). It is possible that a smaller weight quantity of red dye may 


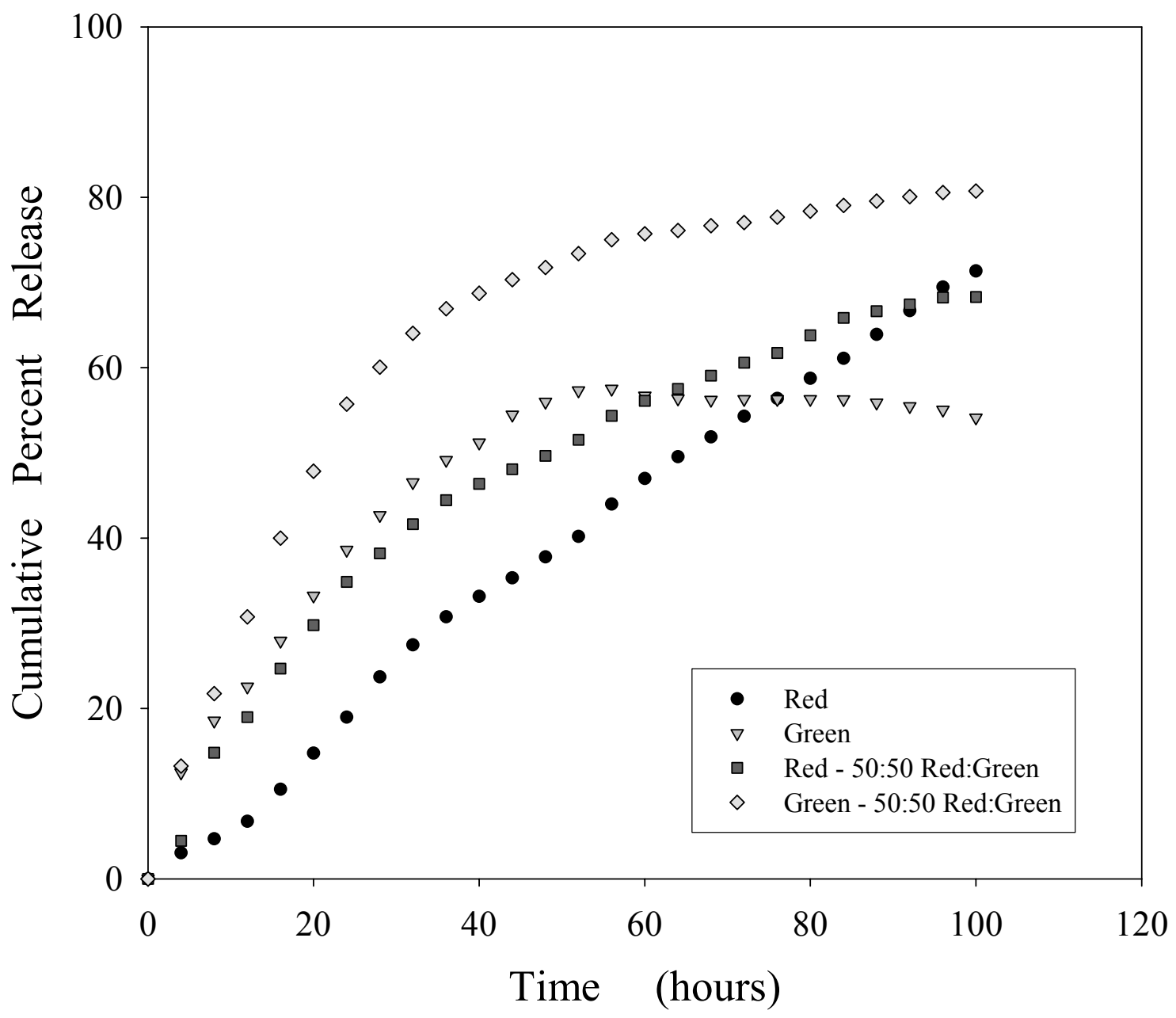

Figure 58. Release profile of non-cross-linked gelatin/ $\mathbf{0 . 0 3 7 \%}$ formaldehyde matrices loaded with red, green, or a combination of red \& green dye.

Matrices were refrigerated for 24 hours followed by 24 hours of lyophilization and evaluated under excess moisture, ambient temperatures, and sink conditions. The results are plotted as an average of triplicate measurements. 
be needed to produce a brightness equivalent to the green dye. The ratio of the red dyes may also be different than the ratio of the blue and yellow components used in the green dye. A second reason for the difference may be that the red dye becomes entangled with the gelatin or cross-linking agents to a greater extent than the blue or yellow components of the dye. This may be due to the bulkiness of the one red component, erythrosine. Thus, red dye diffusion may be more limited. The erythrosine has 4 -iodo groups attached to it which can become entangled within the matrix. As the gelatin strands become mobile, the green dye is able to become free and diffuse out of the matrix. The iodine loaded dye, however, may require more time and a larger number of free strands before it is mobile enough to diffuse through the channels and out of the matrix. Also, a bulkier component may require a longer pathway to get from its original position within the matrix into the solvent. As the erythrosine moves along its path, it may get entangled with another strand or blocked by a new disentangling strand in which a smaller molecule may be better able to diffuse around the strand. The weight of the diffusing molecules may also slow down the diffusion of the red components.

After 6 days of release, $60-80 \%$ of the green dye and $40-60 \%$ of the red dye have been accounted for during dye release. The remaining dye that has not been accounted for may be due to the loss of dye when the samples were blotted dry during the weighing portion of the swelling/bioerosion section of the studies. A second reason for such a difference may be due to dye levels in the sample that were lower than the level of detection. At the end of the 6 days, the change in the concentration in the medium may have been so small that the lamp was unable to detect a change. A third reason for 
unaccountable dye may be due to the retention of the dye through a direct chemical interaction with the gelatin or cross-linking agent. Finally, unaccountable dye may due to the retention of the dye in the matrix. At the six day time interval, there was a significant amount of red dye (20-40\%) still remaining in the matrix, with the green dye retaining 10 to $20 \%$ of the dye. Upon visual analysis of the matrices on day 6 , a slight tint of green was visible within the matrices loaded with green dye. One the other hand, the red loaded matrices demonstrated a bright ring of dye located at the center of all the matrices. It is possible that the transport of the red dye is slowed/stopped due to the loss of its concentration gradient.

A comparison of swelling/erosion and dye release was completed to assess whether the diffusion is the major driving force for release. Figures 59-62 show that, upon exposure to water, the matrices take up a substantial quantity of water within the first 16 hours. The matrices then maintain a constant weight throughout the 6 day study. This indicates that there are two dynamic processes, swelling and erosion taking place during the course of the release profile. The first process is swelling in which the matrices take up water and begin to swell. Eventually, the matrices reach static conditions, in which there is either an equilibrium between swelling and erosion or the matrix ceases to swell (reaches maximum water content) but is not eroding, as the dye continues to be released. Finally, the second process, erosion, causes the gelatin strands to disentangle, bonds to break, and the matrices to fall apart. In addition, the rate of dye release and the swelling rate are different indicating that the two parameters are different. The release profiles show a gradual release of dye over a 100 hour time interval. This 


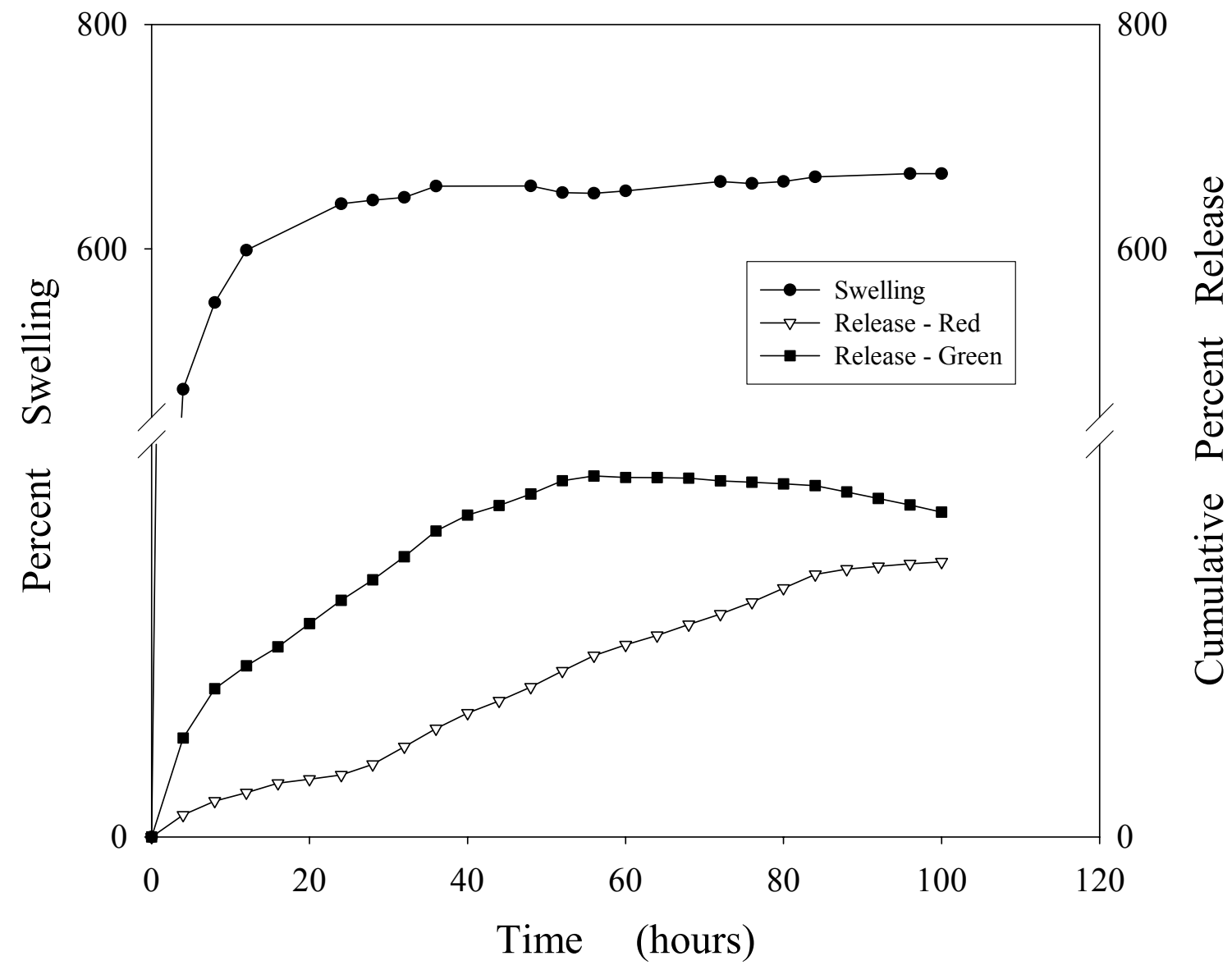

Figure 59. Comparison of the release profile to the swelling/erosion profile for gelatin matrices.

Matrices were refrigerated for 24 hours followed by 24 hours of lyophilization and evaluated under excess moisture, ambient temperatures, and sink conditions. The results are plotted as an average of triplicate measurements. 


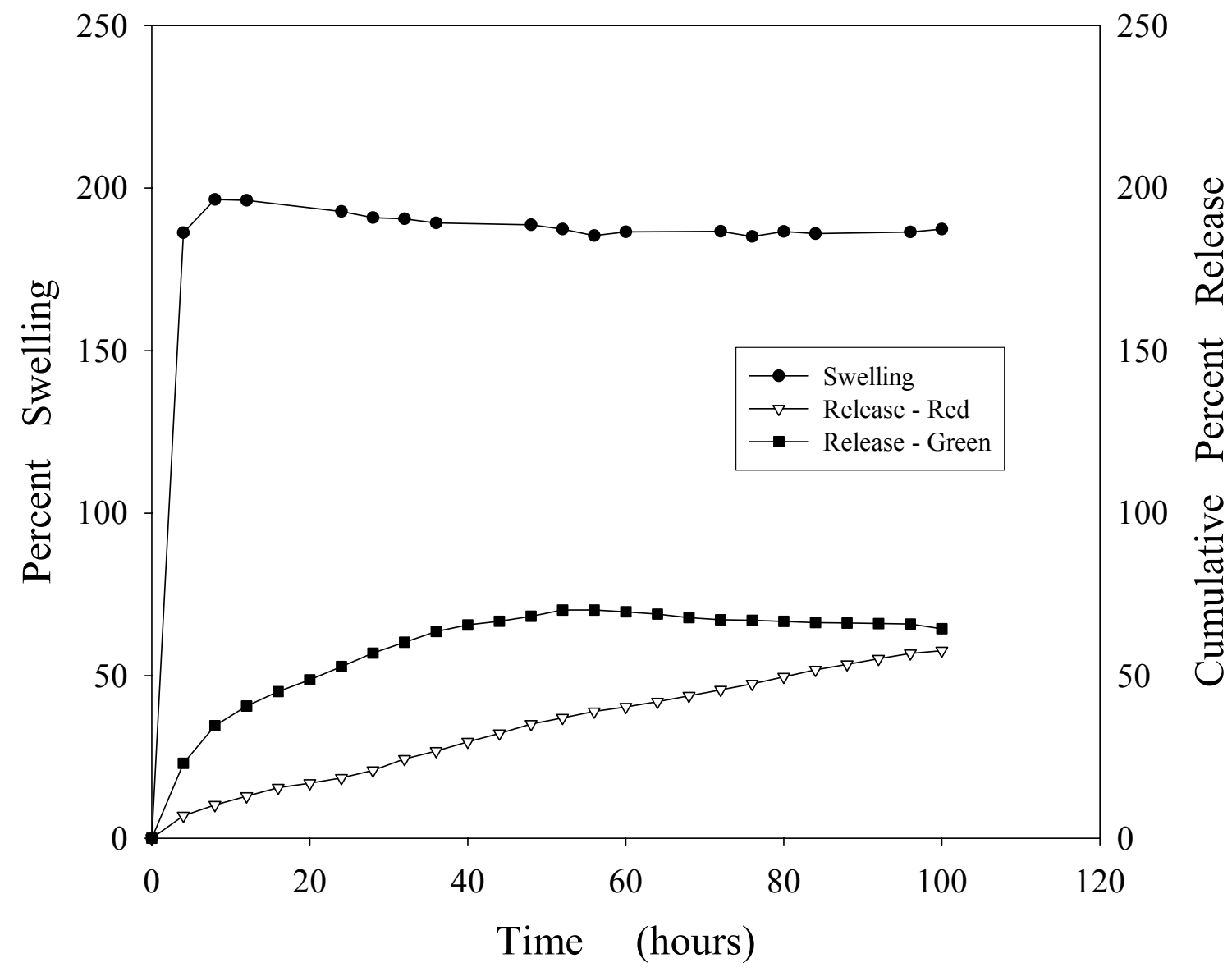

Figure 60. Comparison of the release profile to the swelling/erosion profile for gelatin $/ \mathbf{3 0} \%$ sucrose matrices.

Matrices were refrigerated for 24 hours followed by 24 hours of lyophilization and evaluated under excess moisture, ambient temperatures, and sink conditions. The results are plotted as an average of triplicate measurements. 


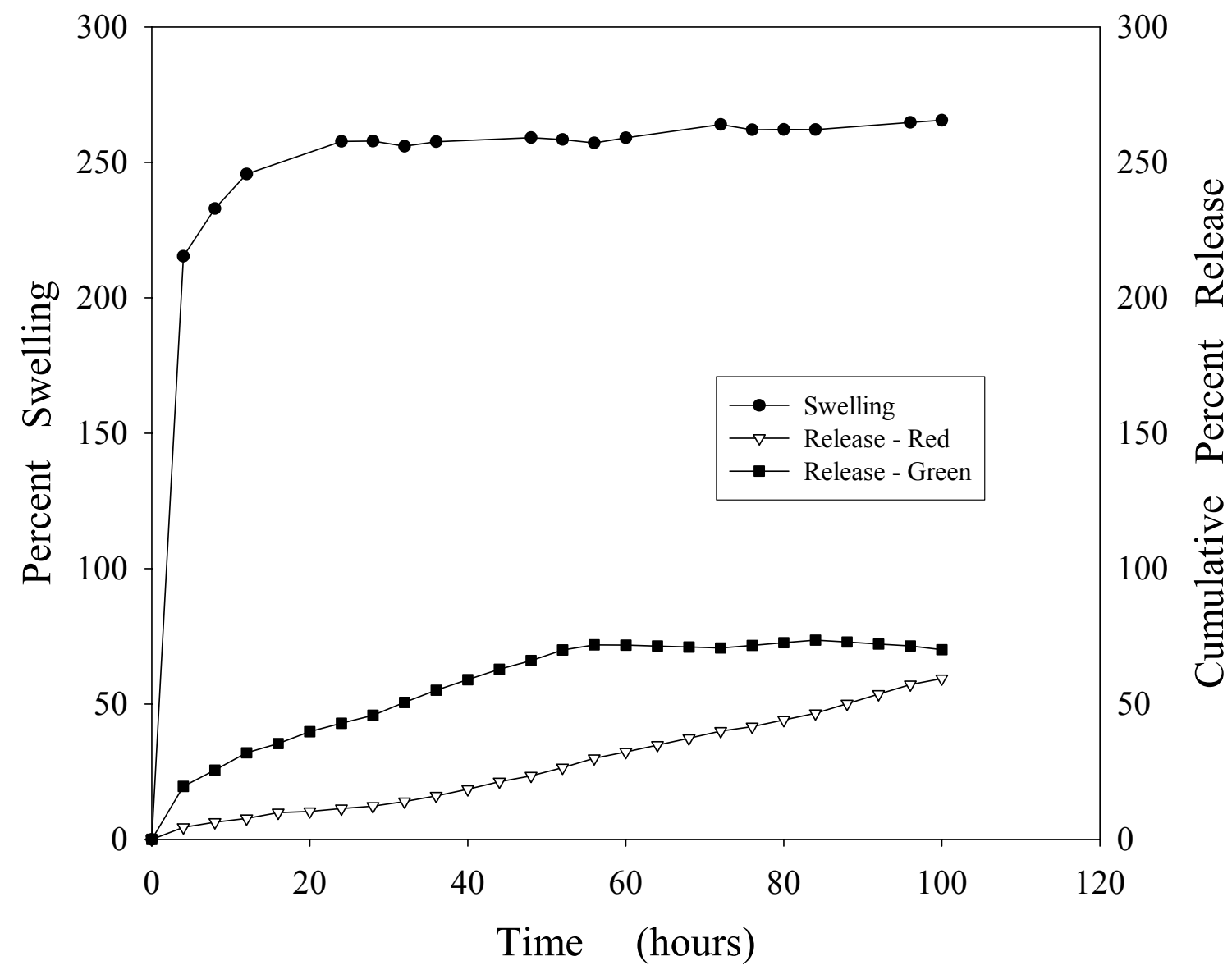

Figure 61. Comparison of the release profile to the swelling/erosion profile for gelatin $\mathbf{1 5 . 8 \%}$ fructose matrices.

Matrices were refrigerated for 24 hours followed by 24 hours of lyophilization and evaluated under excess moisture, ambient temperatures, and sink conditions. The results are plotted as an average of triplicate measurements. 


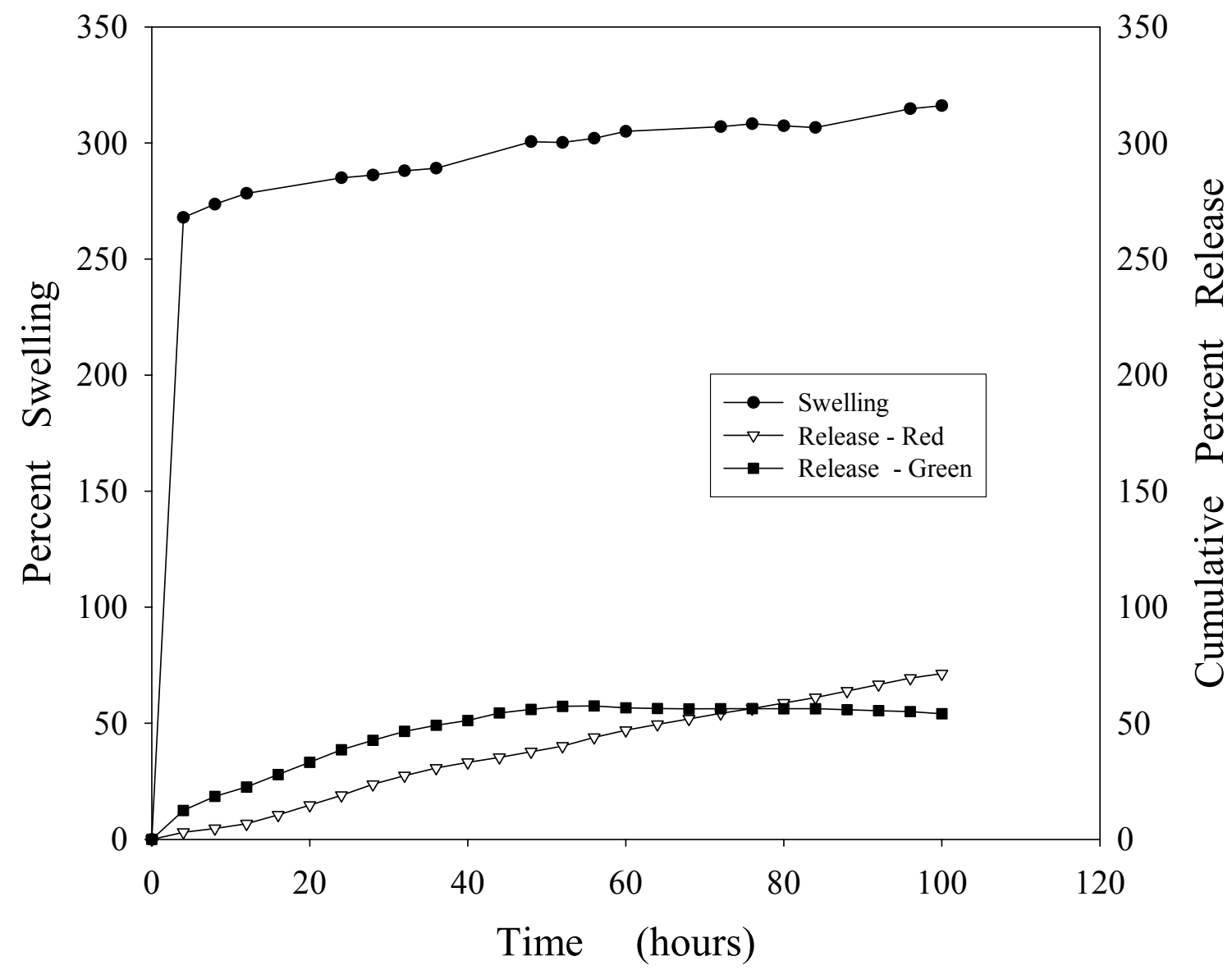

Figure 62. Comparison of the release profile to the swelling/erosion profile for gelatin/0.037\% formaldehyde matrices.

Matrices were refrigerated for 24 hours followed by 24 hours of lyophilization and evaluated under excess moisture, ambient temperatures, and sink conditions. The results are plotted as an average of triplicate measurements. 
indicates that the rate of release is most likely dependent on the concentration that is maintained throughout the release profile. When the matrices release approximately $60 \%$ of the dye loaded, the release rate slows down indicating a loss of the concentration gradient. This confirms the diffusion-controlled process because a swelling controlled soluble matrix swells, reaches a swelling-erosion equilibrium and demonstrates rapid zero order release [7].

\section{Thermal Analysis}

Thermal analysis data shows that the addition of dye (both alone and in combination) produces a stronger, more stable matrix and also appears to dry the disks to a greater extent (Table 8) than without dye. The shift to higher melting temperatures indicates that the addition of the dyes contribute to more rigid polymer sections within the matrix. The addition of sodium salt dyes to the aqueous solution likely causes them to associate with sites on the gelatin, allowing less pore spaces for water during the initial curing process. The dyes have multiple binding sites that can also form cross-links within the matrices, reducing the number of possible weaker hydrogen bond interactions between gelatin and water. The additional bonds that form force the water out creating drier and stronger matrices. This is in agreement with Gautam, et al [8] who reported that the addition of azo dye decreased the moisture content of gelatin films. The authors concluded that the reduced moisture content was due to chemisorption of dyes, indicating that dye binding rendered the gelatin less hydrophilic. This coincides with the decrease seen in the percent weight loss of the gelatin/sucrose matrices due to removal of water in the refrigeration process. Percent weight loss values were calculated using the following 
Table 8. Comparison of $T_{m}$ and \% weight loss of matrices with and without dye.

Data presented here is an average of red, green and combination of dyes and represents a mean of 3 determinations. Matrices were refrigerated for 24 hours followed by 24 hours of lyophilization.

\begin{tabular}{|l|c|c|c|c|c|c|}
\hline & $\begin{array}{c}\text { Temperature } \\
- \text { no dye } \\
\left({ }^{\circ} \mathrm{C}\right)\end{array}$ & $\begin{array}{c}\text { Temperature } \\
- \text { dye } \\
\left({ }^{\circ} \mathrm{C}\right)\end{array}$ & $\begin{array}{c}\text { Additional } \\
\text { Endothermic } \\
\text { Peaks - no } \\
\text { dye }\left({ }^{\circ} \mathrm{C}\right)\end{array}$ & $\begin{array}{c}\text { Additional } \\
\text { Endothermic } \\
\text { Peaks - dye } \\
\left({ }^{\circ} \mathrm{C}\right)\end{array}$ & $\begin{array}{c}\% \text { Wt Loss } \\
- \text { no dye }\end{array}$ & $\begin{array}{c}\text { \% Woss } \\
- \text { dye }\end{array}$ \\
\hline Gelatin & $160-220$ & $210-220$ & $75-80$ & $115-120$ & $77 \%$ & $72 \%$ \\
\hline Gelatin/Sucrose & $150-195$ & $195-200$ & 220 & --- & $45 \%$ & $32 \%$ \\
\hline Gelatin/Fructose & $160-225$ & $180-200$ & --- & --- & $59 \%$ & $54 \%$ \\
\hline $\begin{array}{l}\text { Gelatin/ } \\
\text { Formaldehyde }\end{array}$ & $160-215$ & $190-220$ & $80-90$ & $110-115$ & $77 \%$ & $78 \%$ \\
\hline
\end{tabular}


equation, $\%$ Wt Loss $=\left(m_{o}-m_{1} / m_{o}\right) \times 100$, where $m_{o} \& m_{1}$ are the weights (in $\left.\mathrm{mg}\right)$ of the pre- and post-lyophilization sample, respectively.

\section{Drug Studies}

To further evaluate the gelatin-based matrices, the fluorescein loaded matrices were evaluated to better characterize drug release. According to [6], fluorescein is a freely soluble compound (1-10 parts of solvent for 1 part solute). The swelling and erosion profile shows a rapid burst of fluorescein being released followed by a very slow release for the next 6 days (Figure 63). The rapid burst occurs within the first 20 hours releasing $75-85 \%$ of fluorescein. Little to no difference in release is seen when comparing the gelatin/sugar matrices to the gelatin/formaldehyde matrices (Table 9). The shape of the fluorescein release profile also differs from that of the dyes.

The rapid release of the fluorescein may be due to three factors, molecular size/weight and solubility. Fluorescein is a much smaller compound in both size and weight $(\mathrm{MW}=330)$ than the dye markers $(\mathrm{MW} 500-900)$. These differences may allow the fluorescein to freely diffuse through the matrix without becoming entangled in the gelatin strands or blocked by disentangling strands. The diffusional pathway for the smaller fluorescein may also be shorter due to the increased ability to move freely within the matrix. The increase in solubility may also increase the diffusion of fluorescein due to its ability to become a part of the solvent and move freely within the matrix. The overall net charge of the fluorescein is lower than the net charge of the red or green dye. This may allow the fluorescein molecules to more freely diffuse out of the matrix due to limited interactions with the gelatin strands. 


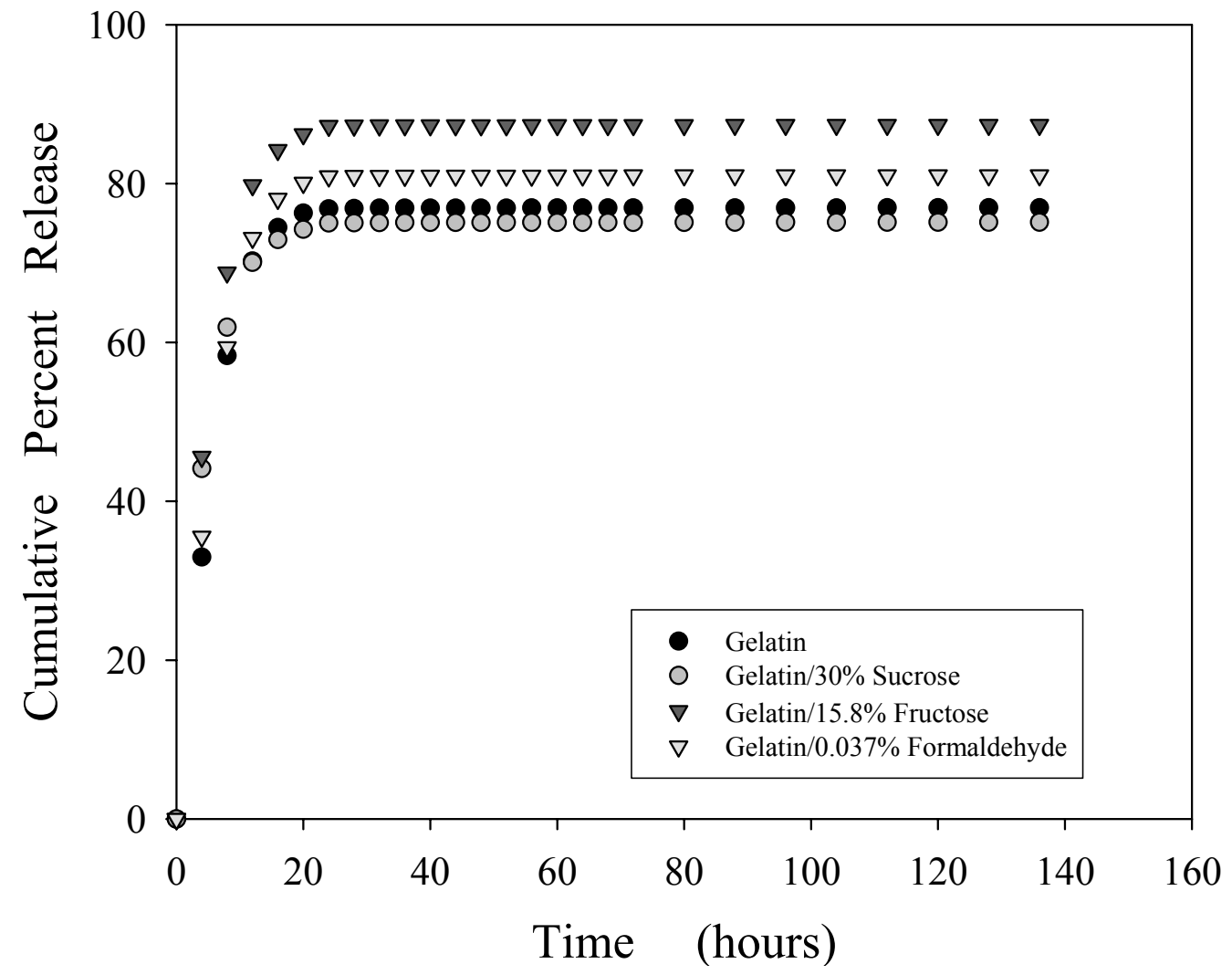

Figure 63. Release profiles of gelatin, gelatin/30\% sucrose, gelatin $/ 15.8 \%$ fructose and gelatin $/ \mathbf{0 . 0 3 7 \%}$ formaldehyde matrices loaded with $0.1 \%$ fluorescein sodium.

Matrices were refrigerated for 24 hours followed by 24 hours of lyophilization and evaluated under excess moisture, ambient temperatures, and sink conditions. The results are plotted as an average of triplicate measurements. 
Table 9. Comparison of the variability of fluorescein release.

Matrices were cured for 3 days at refrigerated temperatures and evaluated under limited conditions and physiological temperatures. Data represents a mean of 3 determinations.

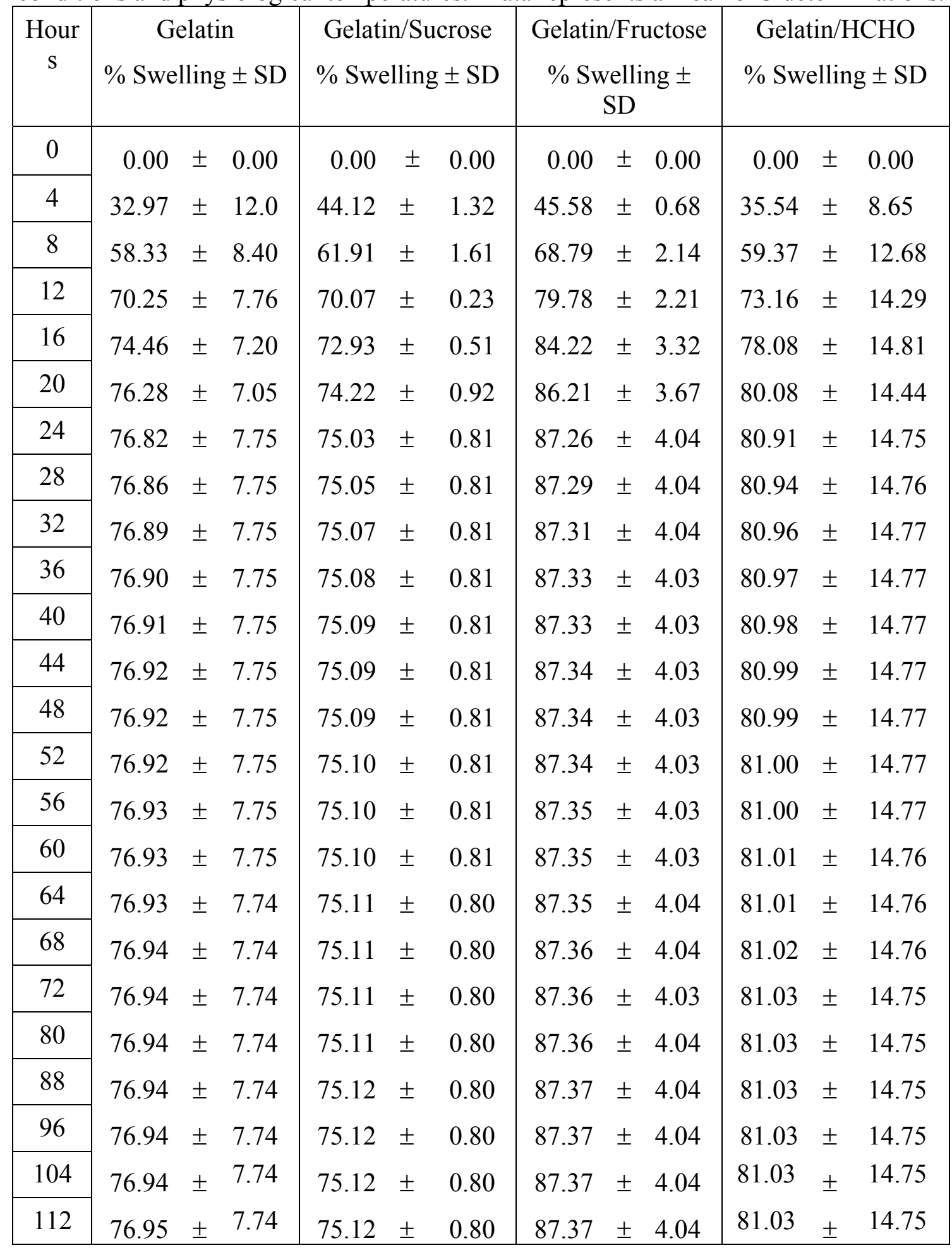


Hämäläinen, et al [9] have shown that fluorescein release from Gelfoam ${ }^{\circledR}$ is diffusion-controlled. The fluorescein studies reported here seem to also confirm that release from these gelatin-based matrices is diffusion-controlled. The rate and extent with which the matrices released fluorescein is much greater than the release of the dyes. The swelling and erosion profiles of the dye loaded and fluorescein loaded matrices are similar (Figures 59-62 for the dyes and Figures 64-67 for fluorescein) indicating that release is not swelling controlled.

When comparing the release profiles of fluorescein to the release profiles of the dye markers, significant differences are seen throughout the study. The release of dye from the matrices is a slow process for the first $8-16$ hours followed by a more rapid release (refer back to Figures 57-60). The release of fluorescein occurs quickly within the first 24 hours followed by slow release (Figure 63). This indicates that the size and charge of the drug molecule may play a major role in the release kinetics from these gelatin-based matrices. The rank order of solubility for fluorescein and the two dyes is fluorescein $>$ green dye $>$ red dye [6]. The path length may also play a role in the release kinetics. A bulkier component (such as in the red dye) may require a longer pathway to get from its original position within the matrix into the solvent. For example, as the erythrosine (a component of the red dye) moves along its path, it may get entangled with another strand or blocked by a new disentangling strand in which a smaller molecule may be better able to diffuse around the strand. The weight of the diffusing molecules may also slow down the diffusion of the dyes. 


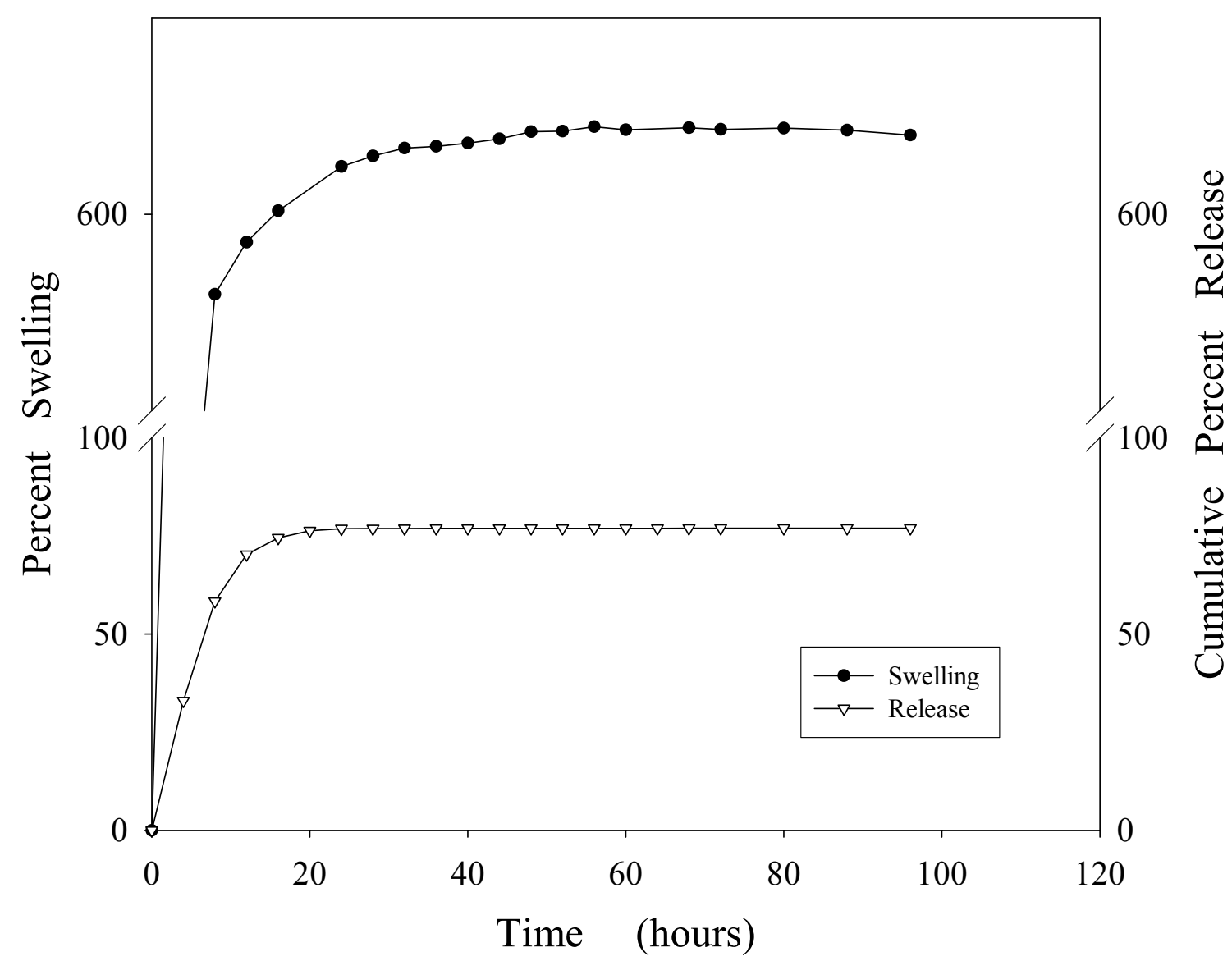

Figure 64. Comparison of the release profile to the swelling/erosion profile for gelatin matrices loaded with fluorescein.

Matrices were refrigerated for 24 hours followed by 24 hours of lyophilization and evaluated under excess moisture, ambient temperatures, and sink conditions. The results are plotted as an average of triplicate measurements. 


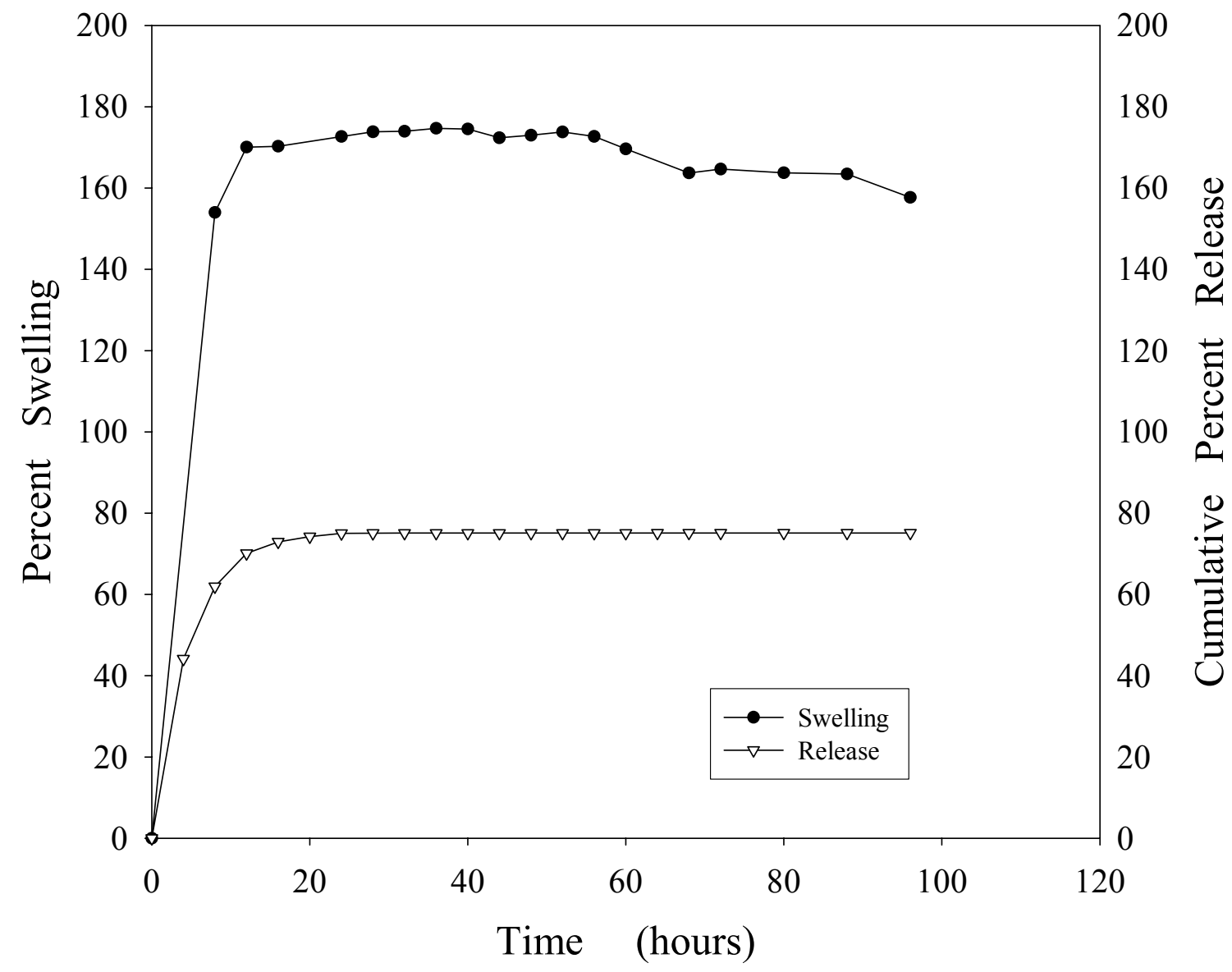

Figure 65. Comparison of the release profile to the swelling/erosion profile for gelatin/30\% sucrose matrices loaded with fluorescein.

Matrices were refrigerated for 24 hours followed by 24 hours of lyophilization and evaluated under excess moisture, ambient temperatures, and sink conditions. The results are plotted as an average of triplicate measurements. 


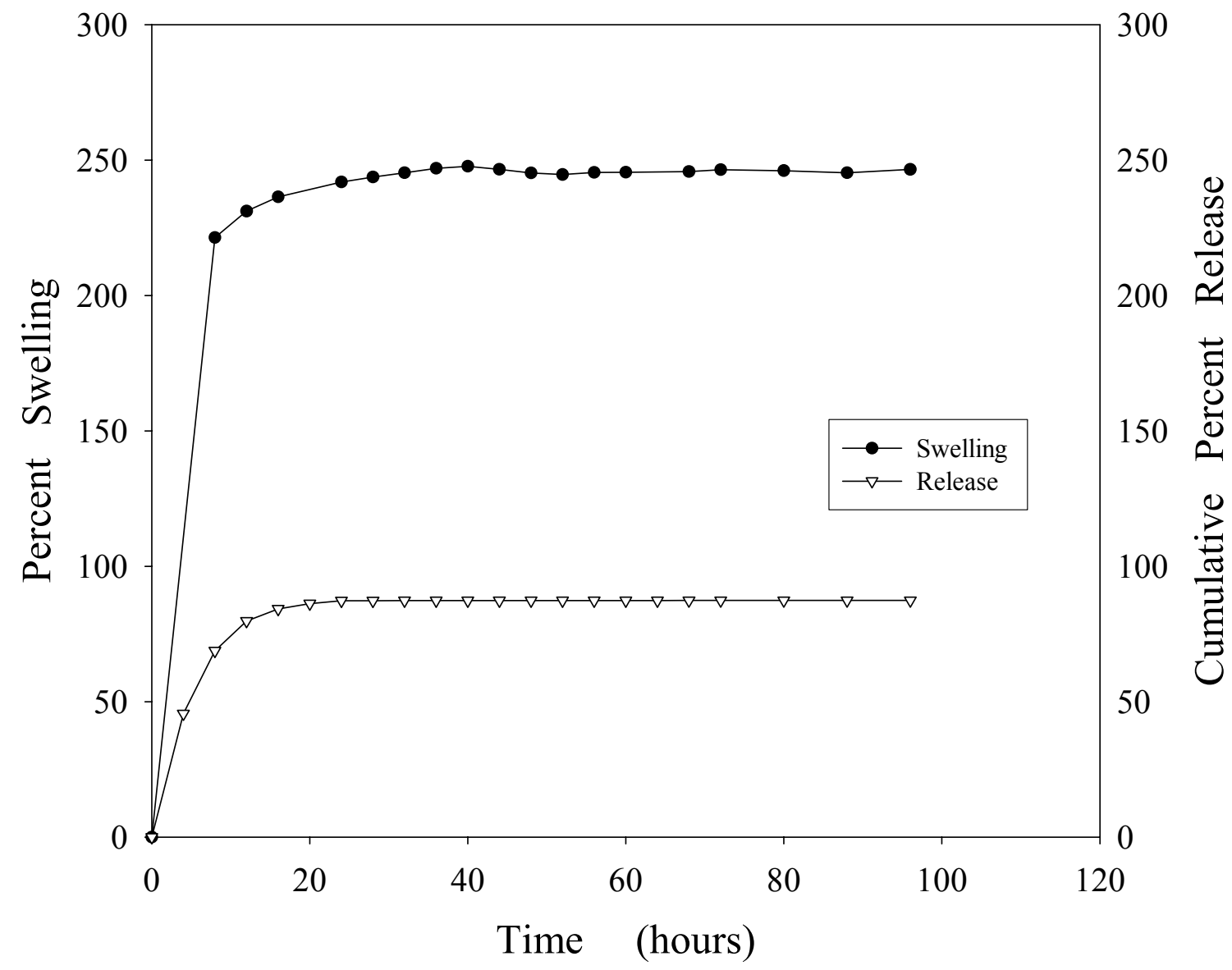

Figure 66. Comparison of the release profile to the swelling/erosion profile for gelatin $\mathbf{1 5 . 8 \%}$ fructose matrices loaded with fluorescein.

Matrices were refrigerated for 24 hours followed by 24 hours of lyophilization and evaluated under excess moisture, ambient temperatures, and sink conditions. The results are plotted as an average of triplicate measurements. 


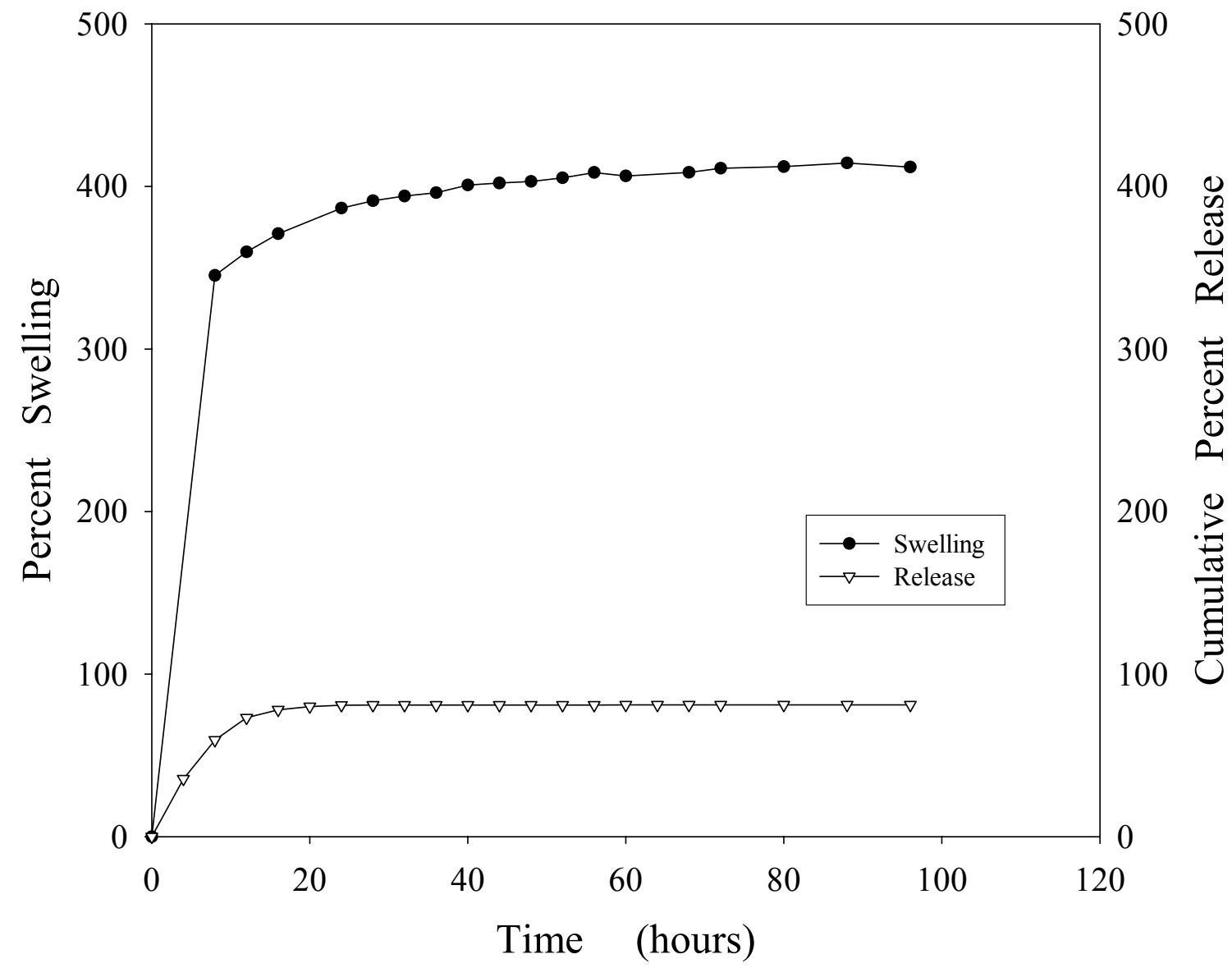

Figure 67. Comparison of the release profile to the swelling/erosion profile for gelatin/0.037\% formaldehyde matrices loaded with fluorescein.

Matrices were refrigerated for 24 hours followed by 24 hours of lyophilization and evaluated under excess moisture, ambient temperatures, and sink conditions. The results are plotted as an average of triplicate measurements. 
A comparison of swelling/erosion and dye release was completed to further confirm that diffusion is the major driving force for release. Figures 64-67 (refer back to previous figures) show that, upon exposure to the water, the matrices take up a substantial quantity of water within the first 12 hours. The matrices then maintain a constant weight throughout the 6 day study. This indicates that there are two dynamic processes, swelling and erosion taking place during the course of the release profile. The first process is swelling in which the matrices take up water and begin to swell. Eventually, the matrices reach static condition, in which there is either an equilibrium between swelling and erosion or the matrix ceases to swell (reaches maximum water content) but is not eroding, as the dye continues to be released. Finally, the second process, erosion, causes the gelatin strands to disentangle, bonds to break, and the matrices to fall apart. The release profile, as described above in this section, also demonstrates a rapid release of fluorescein followed by a second slower release. Due to fluorescein's high solubility, the release of the fluorescein may be influenced by the rate and extent of absorption of water into the matrix. As described by Harland, et al [7], a swelling-controlled soluble matrix swells, reaches a swelling-erosion equilibrium and demonstrates rapid zero order release.

Further studies will need to be done to more fully characterize the release properties of the gelatin, gelatin/sugar, and gelatin/formaldehyde matrices. These studies should include evaluating the release characteristics of prototype drugs that are currently being used in the treatment of periodontal disease as well as ophthalmic disorders. These therapeutic agents would include neutral as well as charged compounds and low and high 
solubility compounds, in order to better assess the influence of the solute's physicochemical properties on solute transport.

\section{Discussion}

Visual release studies revealed that an initial burst (first 8 hours) followed by a gradual release of dye is seen until there is no more dye in the matrix. Visual studies also demonstrated that two different solutes transport out of the matrix at different rates, suggesting a diffusional resistance in the film matrix to solute release.

In vitro release studies revealed that the release of dye from the matrices appears to be diffusion-controlled. This is seen when comparing release under sink to release under non-sink conditions. The evaluation of dye release under non-sink conditions demonstrated a quick release rate for the first 12 hours, at which time the rate decreases over the next 60 hours. On the other hand, dye release under sink conditions demonstrated a gradual release of dye over the entire 72 hours. This indicates that the release of dye is dependent on the concentration gradient and diffusion-controlled. These studies also demonstrated that the green dye releases at a faster rate and to a significantly larger extent than the red dye in both sink and non-sink conditions. Thermal analysis supports that the addition dye enhances the affect the structurally stabilizing effect of the cross-linking agents, both the sugars and formaldehyde. Fluorescein studies confirm that release from the gelatin-based matrices is diffusion-controlled.

The release of the dye and fluorescein suggests that solute release is a function, to some extent, of solute physicochemical properties. These include drug solubility, the salt 
form of the drug, net charge, and the surface area of the solid drug particle. The release profile of fluorescein, a highly soluble sodium salt, shows a rapid burst of fluorescein followed by a slow gradual release. On the other hand, the green and red dyes are less soluble and demonstrated a much slower release over time. The overall net charge of fluorescein is lower than the dyes, which may contribute to the quick release of fluorescein. The number of interactions between the dye and gelatin may be greater than with fluorescein leading to a slower release of the dye.

The rate and extent of swelling does not appear to play a major role in the release of dye from the gelatin-based matrices. The swelling profile indicates that there are two dynamic processes, swelling and erosion, taking place during the release of dye. The first phase is swelling in which the matrices take up water and begin to swell. Eventually, the matrices reach a static condition in which there is an equilibrium between swelling and erosion or the matrix ceases to swell or erode. However, for fluorescein release, the rate and extent of swelling does seem to play a role in the release. The shape of the release profile is a smaller version of the swelling profile. This indicates that swelling may play a role in the release of fluorescein (or other highly soluble drugs) from the gelatin-based matrices. 


\section{References}

1. Chowdhury DK, Mitra AK. Kinetics of in vitro release of a model nucleoside deoxyuridine from crosslinked insoluble collagen and collagen-gelatin maicrospheres. Int J Pharm 1999;193:113-122.

2. Leucuța SE. Controlled release of nifedipine from gelatin microspheres and microcapsules: in vitro kinetics and Pharmacokinetics in man. J Microencap 1990;7(2):209-217.

3. Choi YS, Hong SR, Lee YM, Song KW, Park MH, Nam YS. Study on gelatincontaining artifical skin: I. Preparation and characteristics of novel gelatin-alginate sponges. Biomaterials 1999;20:409-417.

4. Hui X, Pingtian D, Gang W, Guidheng, Z, Junmin Z. Equilibrium swelling and solute diffusion characteristics of poly(methacrylic acid co-poloxamer) hydrogels. J Chin Pharm Sci 2001;10:203-207.

5. Changez M, Burugapalli K, Koul V, Choudhary V. The effect of composition of poly(acrylic acid)-gelatin hydrogel on gentamicin sulphate release: in vitro. Biomaterials 2003;24:527-536.

6. In: Gennero AR, editor. Remington: The science and practice of pharmacy. Baltimore, MD: Lippencott Williams \& Wilkins, year. p. num

7. Harland RS, Gazzaniga A, Sangalli ME, Colombo P, Peppas NA. Drug/polymer matrix swelling and dissolution. Pharm Res 1988;5(8):488-494.

8. Gautam J, Schott H. Interaction of anionic compounds with gelatin II:effect of some physicochemical properties of gelatin. J Pharm Sci 1994;83(3):316-321.

9. Hämäläinen KM, Määttä E, Piirainen K, Sarkola M, Väisänen A, Ranta V-P, Urtti A. Roles of acid/base nature and molecular weight in drug release from matrices of gelfoam and monoisopropyl ester of poly(vinyl methyl ether-maleic anhydride). $J$ Control Rel 1998;56:273-283. 


\section{Chapter VII}

\section{Delivery System Modeling}

There are many factors which can affect the kinetics of drug release from homogeneous polymer matrices. These include physicochemical properties of the drug, size and density of the drug molecule, distribution of the drug, interactions between the drug and matrix, release environment as well as the extent and nature of cross-linking of the matrix [1]. In homogeneous matrix systems, the drug is evenly dispersed throughout the matrix. Drug release from homogeneous matrices assumes that dissolution is initiated at the solid-liquid boundary and, gradually, the front moves through the subsequent layers to the inner core [2]. To analyze the release mechanism of the drug, release data can be fitted into the equation presented by Ritger, et al [3].

$$
\mathrm{M}_{\mathrm{t}} / \mathrm{M}_{\infty}=\mathrm{k} \text { x } t^{n}
$$

where $\mathrm{M}_{t} / \mathrm{M}_{\infty}$ is the fractional release of drug, $t$ is the release time, $\mathrm{k}$ is the release constant, and $n$ is a parameter that represents the type of transport mechanism. As described by Ritger, et al [3], the diffusion exponent, $\mathrm{n}$, for a thin film is defined as $\mathrm{n}=$ 0.5 for Fickian diffusion, $0.5<\mathrm{n}>1.0$ for non-Fickian transport, and $\mathrm{n}=1.0$ for case II transport. Case II transport involves polymer dissolution and chain disentanglement [4].

Diffusion is the main driving force for the release of drugs from many different types of sustained release delivery systems [5]. The rate of drug transport in a diffusional system is dependent on the magnitude of the concentration gradient, $\left(c_{s}-c_{t}\right)$, where $c_{s}$ is the saturated concentration of the drug and $\mathrm{c}_{\mathrm{t}}$ is the concentration at time $t$. Three 
mathematical equations were assessed to model the dissolution profiles of the gelatinbased matrices (a) first order equation (b) Higuchi square root of time equation and (c) Hixson and Crowell cube root equation [2].

a. The first order model is given by

$$
\mathrm{M}_{\mathrm{t}} / \mathrm{M}_{\infty}=1-\mathrm{e}^{-\mathrm{kt}}
$$

b. The Higuchi square root of time model is expressed as

$$
\mathrm{M}_{\mathrm{t}} / \mathrm{M}_{\infty}=\mathrm{kt}^{1 / 2}
$$

c. The Hixson-Crowell cube root model is defined as

$$
\left(1-M_{t} / M_{\infty}\right)^{1 / 3}=1-k t
$$

where $M_{t}$ is the amount of dye released at time $t, M_{\infty}$ is the maximal amount of dye released at infinite time, and $\mathrm{k}$ is the rate constant associated with dye release. These models all predict solute transport as varying functions of concentration. The models do not incorporate elements of either an erosion or swelling model that consider both solvent and matrix effects. All three models assume that sink conditions are maintained throughout the release process. Sink conditions are defined as the state in which the concentration of $c_{t}$ never reaches more than $10-15 \%$ of its maximum solubility[6].

All three models are based on Fick's Law of diffusion which states that the amount of material crossing a unit area, $\mathrm{S}$, of a barrier in time, $t$, is defined as flux. The flux in turn is proportional to the concentration gradient, $d c / d x$ :

$$
\mathrm{J}=-\mathrm{D} d c / d x
$$


where $\mathrm{D}$ is the diffusion coefficient. The concentration gradient can be further simplified to $\mathrm{dC} / d x=\left[\left(\mathrm{c}_{1}-\mathrm{c}_{2}\right) / \mathrm{h}\right]$ where $\mathrm{c}_{1}$ and $\mathrm{c}_{2}$ are the concentration of the drug in the membrane and $\mathrm{h}$ is the membrane thickness.

According to Fick's first law of diffusion [7],

$$
\mathrm{J}=d \mathrm{M} / \mathrm{S} d t=-\mathrm{D} d c / d t
$$

Hence,

$$
d M / d t=(\mathrm{DS} / \mathrm{h})\left(\mathrm{c}_{\mathrm{s}}-\mathrm{c}_{\mathrm{t}}\right) .
$$

If sink conditions hold, then $c_{t}$ is negligible and

$$
d \mathrm{M} / d t=\mathrm{DSc}_{\mathrm{s}} / \mathrm{h}
$$

Integrating the above equation yields

$$
\mathrm{M}=(\mathrm{DS} / \mathrm{h}) \mathrm{c}_{\mathrm{s}} t
$$

If the concentration inside the matrix changes with time, the permeability will follow first order transport [5]

$$
\mathrm{M}_{\mathrm{t}} / \mathrm{M}_{\infty}=1-\mathrm{e}^{-k t}
$$

where $k=(\mathrm{DS} / \mathrm{h}) \mathrm{c}_{\mathrm{s}}$.

In order for this model to hold true throughout drug release, several assumptions must hold true throughout drug release. The first assumption is that sink conditions are maintained throughout the course of drug release, in which the volume of the dissolution medium is large or the dissolution medium is replaced constantly with fresh solvent so the solute never reaches more than $10-15 \%$ of its maximum solubility. A second assumption is that the concentration gradient inside the matrix changes with time. If this 
does not occur, then the matrix would follow zero order release kinetics. A third assumption is that the surface area of the particle remains constant throughout the dissolution process.

Higuchi developed an equation for the release of drug dispersed in a homogeneous matrix. As a drug moves out of a matrix, the boundary between the drug and the matrix recedes with time and the thickness of the empty matrix, $d h$, increases with time [7]. The infinitesimal amount, $d Q$, of drug released because of this shift is given by

$$
d Q=\mathrm{A} d h-1 / 2 \mathrm{c}_{\mathrm{s}} d h
$$

where $\mathrm{A}$ is the total amount (dissolved and undissolved) of drug in the matrix. Substituting this equation into Fick's law [7],

$$
d M / \mathrm{S} d t=d Q / d t=\mathrm{Dc}_{\mathrm{S}} / \mathrm{h}
$$

inetegrating and solving for $\mathrm{h}$ gives

$$
\mathrm{h}=\left(4 \mathrm{Dc} \mathrm{c}_{\mathrm{s}} \mathrm{t} / 2 \mathrm{~A}-\mathrm{c}_{\mathrm{s}}\right)^{1 / 2}
$$

The amount of drug depleted per unit area of the matrix, $\mathrm{Q}$, at time $t$, is obtained by integrating equation 8 yielding

$$
\mathrm{Q}=\mathrm{hA}-1 / 2 \mathrm{hc}_{\mathrm{s}}
$$

Substituting equation 10 into equation 11 yields

$$
\mathrm{Q}=\left(\mathrm{Dc}_{\mathrm{s}} \mathrm{t} / 2 \mathrm{~A}-\mathrm{c}_{\mathrm{s}}\right)^{1 / 2}\left(2 \mathrm{~A}-\mathrm{c}_{\mathrm{s}}\right)
$$

Rearrangement of this equation results in the Higuchi equation [8]:

$$
\mathrm{Q}=\left[\mathrm{D}\left(2 \mathrm{~A}-\mathrm{c}_{\mathrm{s}}\right) \mathrm{c}_{s} t\right]^{1 / 2}
$$


Further rearrangement of this equation gives

$$
\mathrm{M}_{\mathrm{t}} / \mathrm{M}_{\infty}=\mathrm{k} t^{1 / 2}
$$

As with the first order model, the Higuchi model is based on several assumptions. The first assumption is that in homogenous matrix systems, drug diffusion occurs as a slow two step process. The first step process involves the initial release of the drug molecules in the periphery of the matrix. Drug molecules from the center of the matrix then need to diffuse to the periphery of the matrix before the drug can be released. The release profile therefore follows a square-root-of-time relationship for the mass of drug released and an inverse square root of time dependence for the drug release rate, as described by the Higuchi equation. The second assumption is that sink conditions are maintained throughout the course of drug release, in which the volume of the dissolution medium is large or the dissolution medium is replaced constantly with fresh solvent so the solute never reaches more than $10-15 \%$ of its maximum solubility. A third assumption is that the amount of drug dispersed in the polymer is greater than the drug solubility in the polymer. A pseudo-steady state is also assumed, in which a linear concentration gradient from the solid/dissolved drug interface to the releasing surface is maintained throughout the drug release. A final assumption of the Higuchi model is that the drug particle size is much smaller than the polymer thickness.

Based on Fick's second law, Noyes and Whitney developed an equation to describe the phenomena of dissolution [6]:

$$
d c / d t=\mathrm{K}\left(\mathrm{c}_{\mathrm{s}}-\mathrm{c}_{\mathrm{t}}\right)
$$


where $\mathrm{K}$ is the proportionality constant. This equation is limited by the assumption that the film maintains a constant surface area. Brunner and Tolloczko modified the NoyesWhitney equation to incorporate the surface area as a separate variable. The Brunner and Tolloczko equation is described as

$$
d c / d t=\mathrm{k}_{1} \mathrm{~S}\left(\mathrm{c}_{\mathrm{s}}-\mathrm{c}_{\mathrm{t}}\right)
$$

where $\mathrm{S}$ is the surface area and $\mathrm{k}_{1}$ is a rate constant [6]. Hixson-Crowell further developed the Brunner-Tolloczko equation to create a dissolution equation that was based on a changing surface area. Hixson and Crowell modified the equation to represent the rate of appearance of the solute in solution by multiplying each side of the equation by $\mathrm{v}$ (volume)

$$
d \mathrm{M} / d t=\mathrm{KS}\left(\mathrm{c}_{\mathrm{s}}-\mathrm{c}_{\mathrm{t}}\right)
$$

where $\mathrm{K}=\mathrm{k}_{1} \mathrm{v}, \mathrm{S}$ is the surface area, and $\mathrm{M}$ is the weight of the solute in solution. Assuming $\mathrm{S}=\mathrm{km}^{2 / 3}$ where $\mathrm{k}$ is a constant containing the shape factor and the density of the particle, and $\mathrm{m}$ is the weight of undissolved particles at time $t[6]$.

$$
d \mathrm{M} / d t=\mathrm{K}\left(\mathrm{km}^{2 / 3}\right)\left(\mathrm{c}_{\mathrm{s}}-\mathrm{c}_{\mathrm{t}}\right)
$$

Applying Fick's first law and further integration of the above equation results in the Hixson-Crowell Cube Root Equation

$$
\mathrm{M}_{\mathrm{o}}^{1 / 3}-\mathrm{M}_{\mathrm{t}}^{1 / 3}=\mathrm{K}_{1} t
$$

which can be further reduced to

$$
\left(1-\mathrm{M}_{\mathrm{t}} / \mathrm{M}_{\infty}\right)^{1 / 3}=1-\mathrm{kt}
$$

The Hixson-Crowell method is a model which was further developed from the NoyesWhitney and Fick's law of diffusion and is based on the same assumptions as the first 
order model with one exception. The Hixson-Crowell model assumes that the radius of the particle is not constant. This leads to the addition of a constant containing the shape factor as well as the density of the particle.

\section{Methodology}

Phase IV (Color and Drug Loading Studies) data along with swelling and erosion data from Phases I \& II were used to assess the influence of matrix properties and the nature of the dye and fluorescein on release in order to identify and model those mechanisms controlling drug release from the optimized formulations. This data was also used to select the matrix which best performs relative to sustained release criteria, and guide any final changes made to its formulation design.

\section{Results}

Based on the three models presented in the beginning of this chapter, the Higuchi model seems best suited for describing the release of dye from the gelatin-based matrices. The Higuchi model describes the release of drug from a polymer matrix while the other two models describe the dissolution of the drug particles. The first order and HixsonCrowell models do not take into account the path length in which the drug must travel in order to be released from the matrix. The Higuchi model also takes into account that release from the matrix follows a two step process. The two step process is based on the dissolution of the solid drug particles followed by the release of the drug from the matrix. This correlates with the visual data, presented in the previous chapter, which has shown an initial release followed by a second release. The removal of water from the matrix 
during the lyophilization process may cause the precipitation of the dye and fluorescein. Upon wetting of the matrix, the particles will then need to first dissolve into the solution in order to be released, resulting in a two step process.

\section{Non-Sink Conditions}

The non-sink release data demonstrates a biphasic pattern of release under the conditions used in the in vitro study (Figures $68 \& 69$ ). Further analysis indicates that the release profile can be reduced to three different release rates, drug release during a swelling phase followed by two diffusional phases, with changing dye release rates. The initial rate, which occurs during the first 3 hours, is related to the initial swelling phase of the matrix when first exposed to the water medium. As the surface of the matrix is exposed to the water, it is hypothesized that the free dye on the matrix surface (possibly concentrated by the syneresis process) and solvent convection effects transport dye out of the matrix. For dye within the matrix pores or associated with the gelatin, a diffusion process becomes the main driving force, resulting in the second phase occurring between 4-11 hours in the green matrices and at 4-12 hours in the red matrices.

The third phase (24-64 hours) is a slow diffusion phase, possibly due to a less favorable concentration gradient, that ultimately results in a much slower release rate. Upon exposure to the dissolution medium, the dye closest to the edges diffuse out first, leaving the dye embedded deep in the matrix. As the water diffuses into the matrix, it must pass through the matted arrangement of gelatin strands (which create a gelatinous diffusional barrier), physically separate some strands, and create a pathway (pore space) for the dye to diffuse out through. This leads to a delay in the release of the dye 


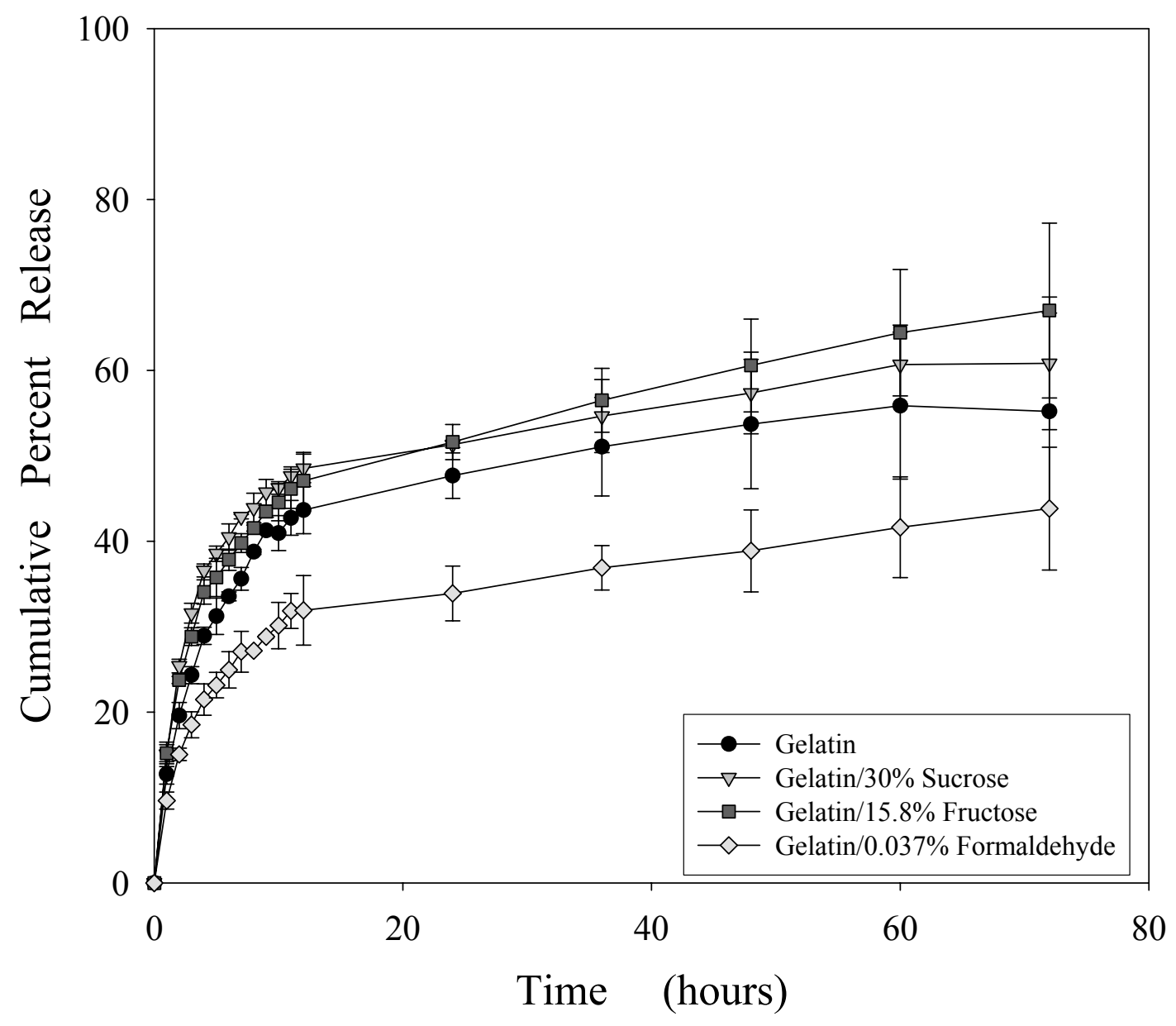

Figure 68. Release profile of gelatin, gelatin/30\% sucrose, gelatin/15.8\% fructose, and gelatin $/ \mathbf{0 . 0 3 7 \%}$ formaldehyde matrices loaded with green dye.

Matrices were refrigerated for 24 hours followed by 24 hours of lyophilization and evaluated under excess moisture and ambient temperatures. The dissolution medium was not changed throughout the study. The results are plotted as an average of triplicate measurements. 


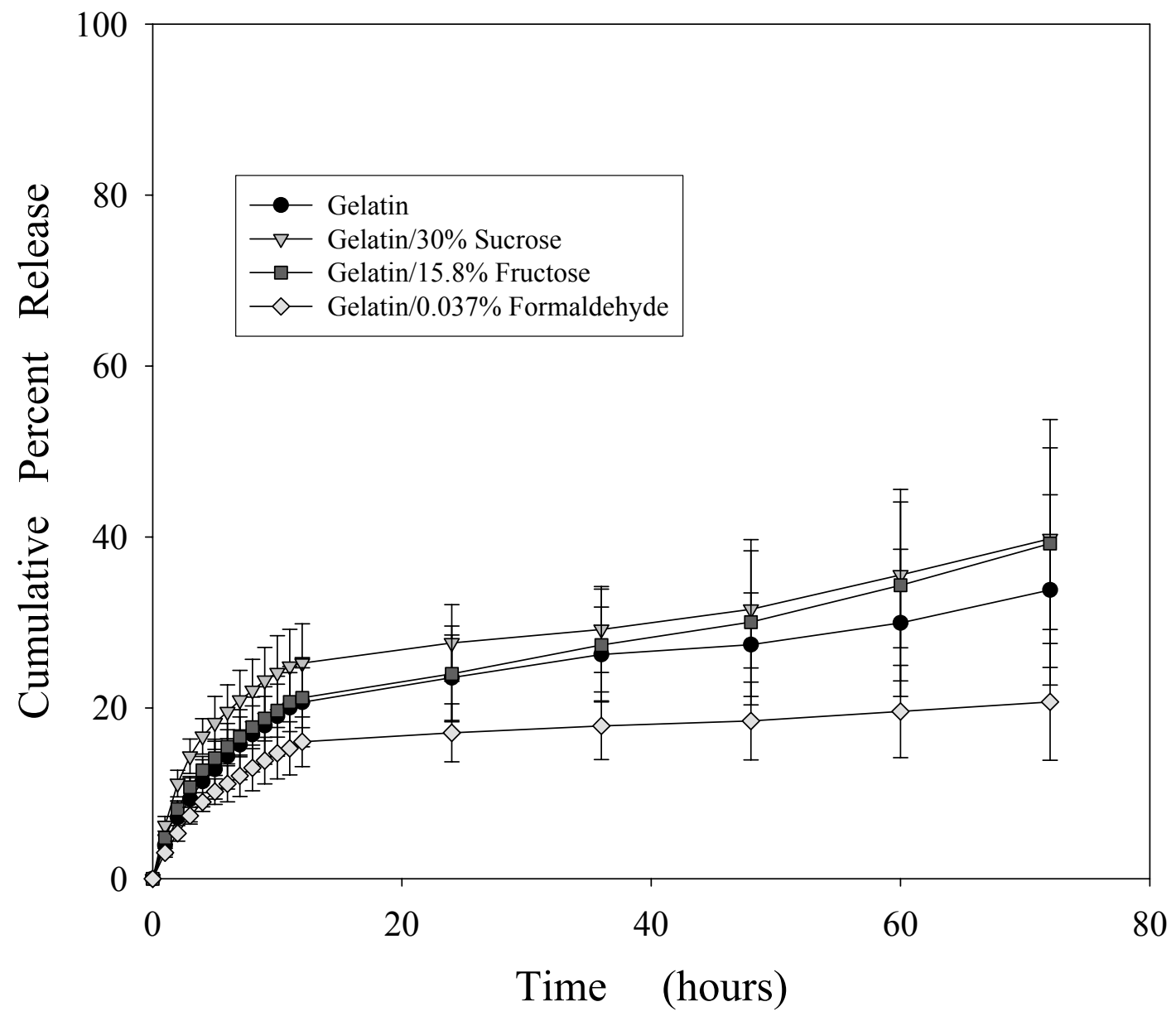

Figure 69. Release profile of gelatin, gelatin/30\% sucrose, gelatin/15.8\% fructose, and gelatin/ $\mathbf{0 . 0 3 7 \%}$ formaldehyde matrices loaded with red dye.

Matrices were refrigerated for 24 hours followed by 24 hours of lyophilization and evaluated under excess moisture and ambient temperatures. The dissolution medium was not changed throughout the study. The results are plotted as an average of triplicate measurements. 
embedded in the matrix. It is likely that both a diminishing concentration gradient, as well as an increased resistance to the dye's outward diffusion (as the matrix wets and gels) slows the dye's release in the third phase. Net charge interaction of the solute and polyionic gelatin may also play a role in this. The study design required only a small amount $(15 \mu \mathrm{l})$ of the total medium volume $(3 \mathrm{ml})$ to be changed at every time point. Essentially, the bulk of the dissolution medium was not changed throughout the course of the studies, leading to a build-up of dye in the bulk. This is supported by qualitative visual data. Eventually, the water medium could contain more dye than the matrix, slowing down the release rate of the dye. The concentration of the dyes is unknown due to the use of pre-formulated dyes from McCormick \& Co, Inc (Hunt Valley, MD). The dyes are available as a solution in an unknown ratio of the dye components.

After fitting the in vitro release data to the three equations, the correlation coefficients indicate that the best fit is obtained with the Higuchi diffusional model rather than the first order or Hixson-Crowell cube root equation, as would be expected. Tables 10 and 11 suggest that the dye release follows a simple diffusional mechanism. The regression for the three models was evaluated and demonstrated good correlations, ranging from 0.93 to 1.00 . However, based on the three models presented in the introduction of this chapter, the Higuchi model best predicts the release of dye from the non-cross-linked gelatin, gelatin/sucrose, gelatin/fructose, and gelatin/formaldehyde matrices (Figures 70-81). The first order release model and the Hixson-Crowell equation describe the dissolution of drug particles and do not consider release from a polymer matrix. 
Table 10. Correlation coefficients of different mathematical models for green dye.

Data represents a mean of 3 determinations. HCHO represents formaldehyde.

\begin{tabular}{|c|ccc|}
\hline GELATIN & $\begin{array}{c}\text { First Order } \\
\text { Model }\end{array}$ & $\begin{array}{c}\text { Higuchi Square Root } \\
\text { of Time Model }\end{array}$ & $\begin{array}{c}\text { Hixson-Crowell Cube Root } \\
\text { Equation }\end{array}$ \\
\hline Hours 1-3 & 0.9651 & 0.9989 & 0.9918 \\
Hours 4-12 & 0.9812 & 0.9971 & 0.9658 \\
& & 0.9989 & 0.9924 \\
\hline
\end{tabular}

\begin{tabular}{|c|c|c|c|}
\hline $\begin{array}{c}\text { GELATIN/ } \\
\text { SUCROSE }\end{array}$ & $\begin{array}{c}\text { First Order } \\
\text { Model }\end{array}$ & $\begin{array}{c}\text { Higuchi Square Root } \\
\text { of Time Model }\end{array}$ & $\begin{array}{c}\text { Hixson-Crowell Cube Root } \\
\text { Equation }\end{array}$ \\
\hline Hours 1-3 & 0.9468 & 0.9947 & 0.9847 \\
& & 0.9912 & 0.9794 \\
Hours 4-12 & 0.9618 & 0.9965 & 0.9940 \\
\hline Hours 24-64 & 0.9672 & & \\
\hline
\end{tabular}

\begin{tabular}{|c|c|c|c|}
\hline $\begin{array}{c}\text { GELATIN/ } \\
\text { FRUCTOS } \\
\text { E }\end{array}$ & $\begin{array}{c}\text { First Order } \\
\text { Model }\end{array}$ & $\begin{array}{c}\text { Higuchi Square Root } \\
\text { of Time Model }\end{array}$ & $\begin{array}{c}\text { Hixson-Crowell Cube Root } \\
\text { Equation }\end{array}$ \\
\hline Hours 1-3 & 0.9507 & 0.9944 & 0.9831 \\
Hours 4-12 & 0.9818 & 0.9976 & 0.9938 \\
Hours 24-64 & 0.9777 & 0.9983 & 0.9946 \\
\hline
\end{tabular}

\begin{tabular}{|c|c|c|c|}
\hline $\begin{array}{c}\text { GELATIN/ } \\
\text { HCHO }\end{array}$ & $\begin{array}{c}\text { First Order } \\
\text { Model }\end{array}$ & $\begin{array}{c}\text { Higuchi Square Root } \\
\text { of Time Model }\end{array}$ & $\begin{array}{c}\text { Hixson-Crowell Cube Root } \\
\text { Equation }\end{array}$ \\
\hline Hours 1-3 & 0.9576 & 0.9970 & 0.9866 \\
Hours 4-12 & 0.9908 & 0.9944 & 0.9802 \\
Hours 24-64 & 0.9572 & 0.9937 & 0.9947 \\
\hline
\end{tabular}


Table 11. Correlation coefficients of different mathematical models for red dye.

Data represents a mean of 3 determinations. HCHO represents formaldehyde.

\begin{tabular}{|c|ccc|}
\hline GELATIN & $\begin{array}{c}\text { First Order } \\
\text { Model }\end{array}$ & $\begin{array}{c}\text { Higuchi Square Root } \\
\text { of Time Model }\end{array}$ & $\begin{array}{c}\text { Hixson-Crowell Cube Root } \\
\text { Equation }\end{array}$ \\
\hline Hours 1-3 & 0.9515 & 0.9980 & 0.9879 \\
Hours 4-11 & 0.9752 & 0.9997 & 0.9952 \\
& & 0.9943 & 0.9848 \\
\hline
\end{tabular}

\begin{tabular}{|c|c|c|c|}
\hline $\begin{array}{c}\text { GELATIN/ } \\
\text { SUCROSE }\end{array}$ & $\begin{array}{c}\text { First Order } \\
\text { Model }\end{array}$ & $\begin{array}{c}\text { Higuchi Square Root } \\
\text { of Time Model }\end{array}$ & $\begin{array}{c}\text { Hixson-Crowell Cube Root } \\
\text { Equation }\end{array}$ \\
\hline Hours 1-3 & 0.9500 & 0.9973 & 0.9867 \\
Hours 4-11 & 0.9761 & 0.9986 & 0.9916 \\
& & 0.9326 & 0.9661 \\
Hours 12-64 & 0.9840 & & \\
\hline
\end{tabular}

\begin{tabular}{|c|c|c|c|}
\hline $\begin{array}{c}\text { GELATIN/ } \\
\text { FRUCTOSE }\end{array}$ & $\begin{array}{c}\text { First Order } \\
\text { Model }\end{array}$ & $\begin{array}{c}\text { Higuchi Square Root } \\
\text { of Time Model }\end{array}$ & $\begin{array}{c}\text { Hixson-Crowell Cube Root } \\
\text { Equation }\end{array}$ \\
\hline Hours 1-3 & 0.9671 & 1.0000 & 0.9950 \\
Hours 4-11 & 0.9794 & 0.9999 & 0.9958 \\
Hours 12-64 & 0.9974 & 0.9684 & 0.9905 \\
\hline
\end{tabular}

\begin{tabular}{|c|c|c|c|}
\hline $\begin{array}{c}\text { GELATIN/ } \\
\text { FORMALD } \\
\text { EHYDE }\end{array}$ & $\begin{array}{c}\text { First Order } \\
\text { Model }\end{array}$ & $\begin{array}{c}\text { Higuchi Square Root } \\
\text { of Time Model }\end{array}$ & $\begin{array}{c}\text { Hixson-Crowell Cube Root } \\
\text { Equation }\end{array}$ \\
\hline Hours 1-3 & 0.9788 & 0.9979 & 0.9996 \\
Hours 4-11 & 0.9779 & 0.9991 & 0.9952 \\
Hours 12-64 & 0.9883 & 0.9837 & 0.9900 \\
\hline
\end{tabular}




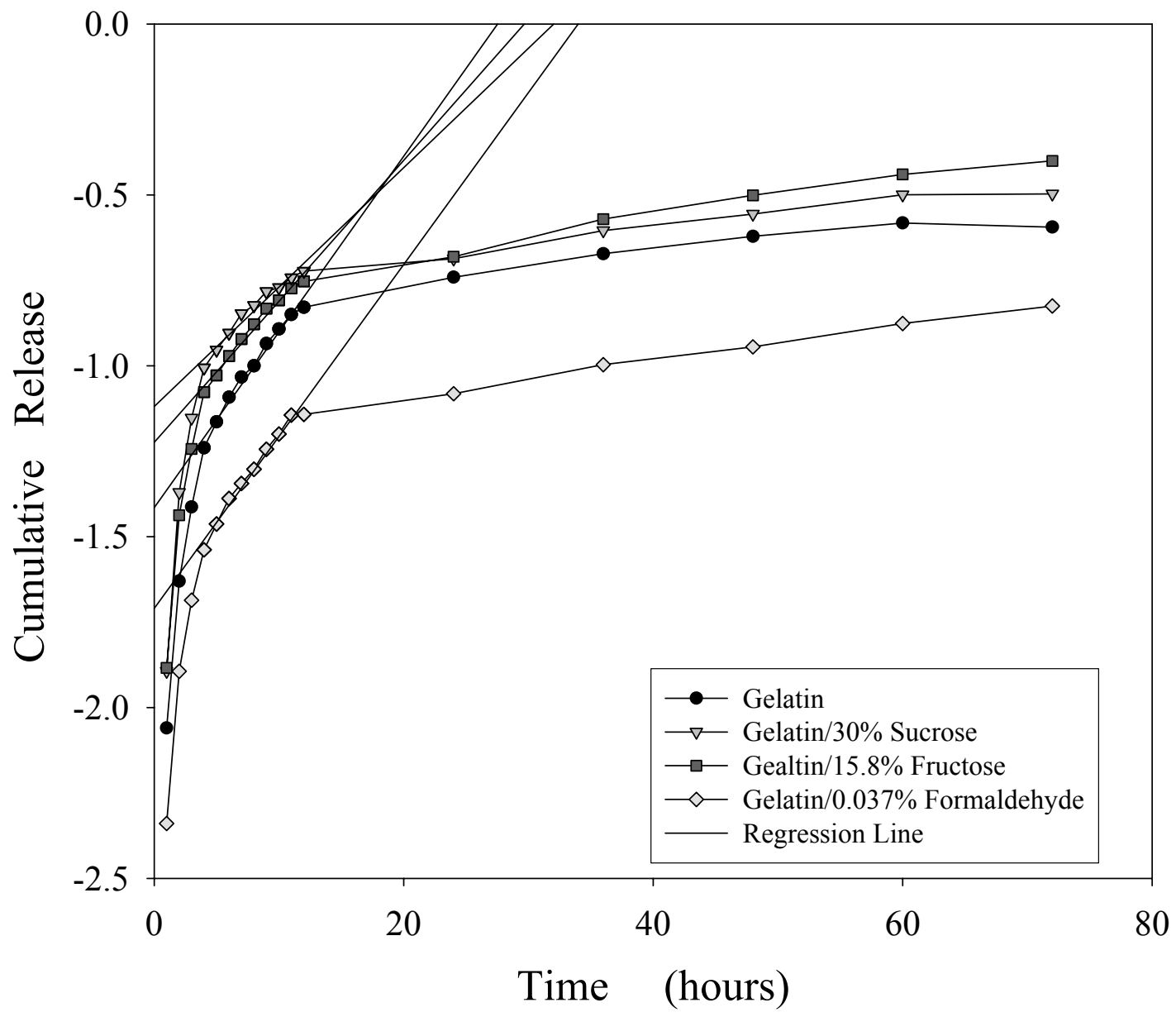

Figure 70. Linear regression for the first order equation over the second release phase (Hours 4-12) for matrices loaded with green dye.

Matrices were refrigerated for 24 hours followed by 24 hours of lyophilization and evaluated under excess moisture and ambient temperatures. The dissolution medium was not changed throughout the study. The results are plotted as an average of triplicate measurements. 


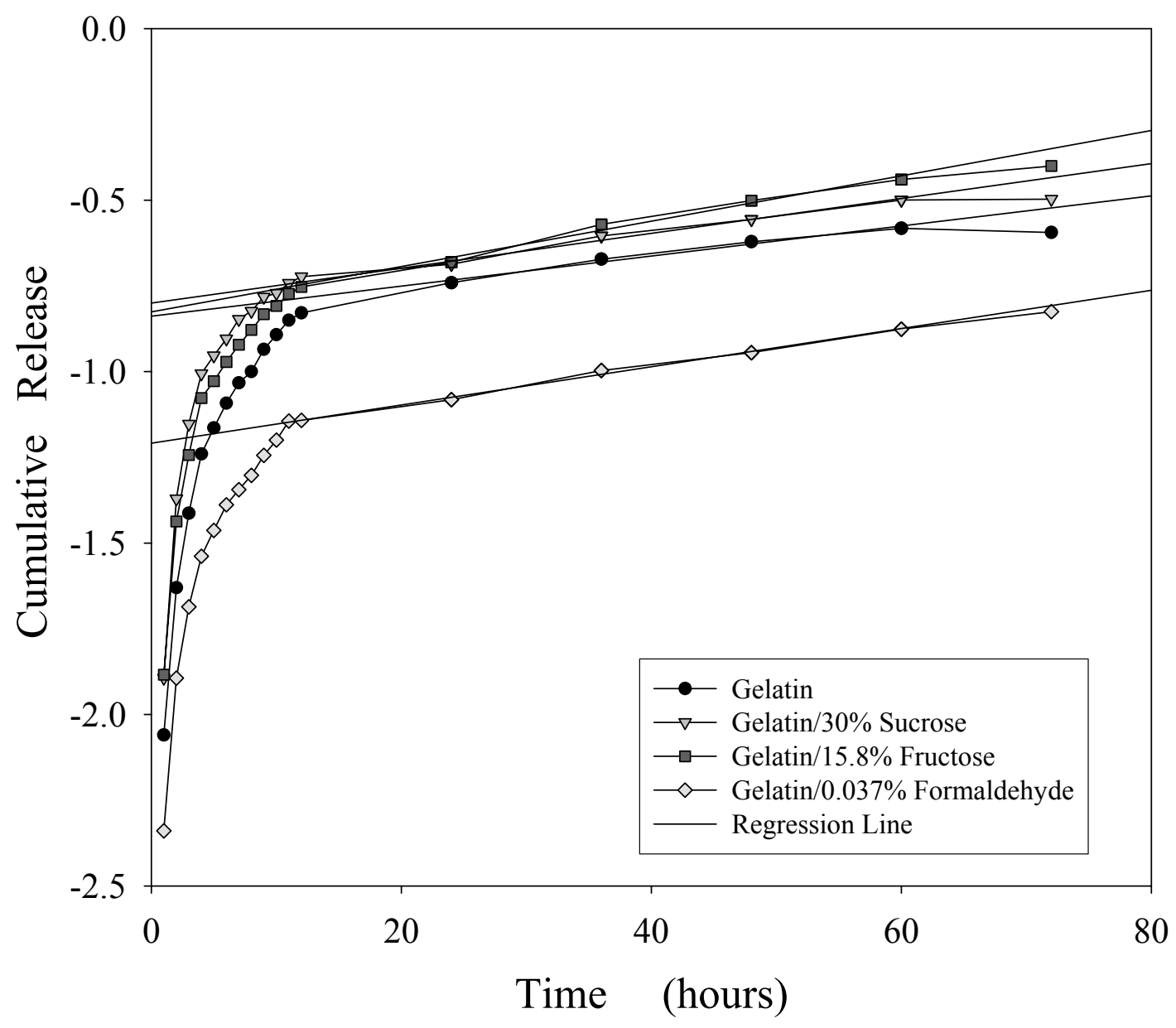

Figure 71. Linear regression for the first order equation over the third release phase (Hours 24-64) for matrices loaded with green dye.

Matrices were refrigerated for 24 hours followed by 24 hours of lyophilization and evaluated under excess moisture and ambient temperatures. The dissolution medium was not changed throughout the study. The results are plotted as an average of triplicate measurements. 


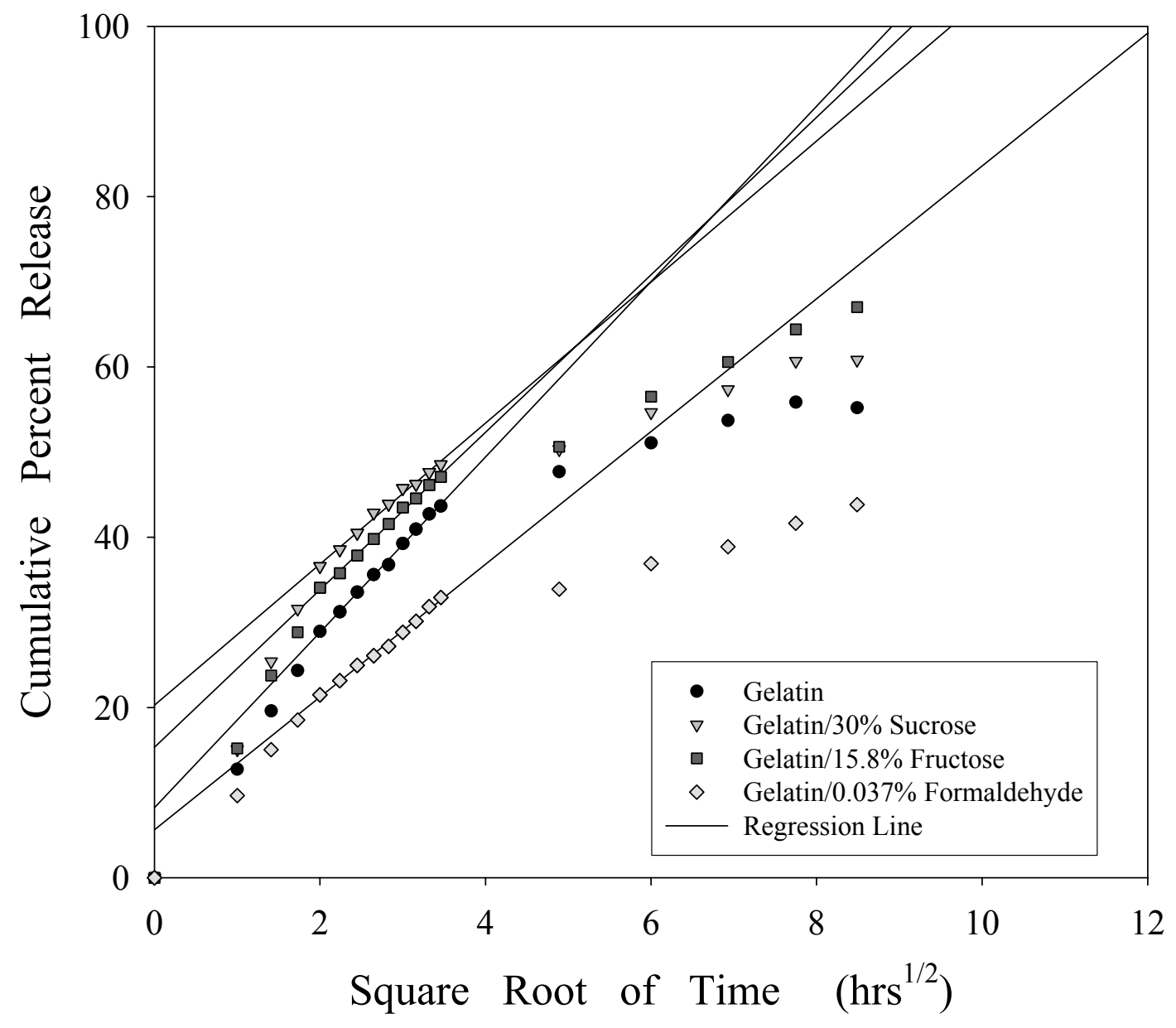

Figure 72. Linear regression for the Higuchi square root equation over the second release phase (Hours 4-12) for matrices loaded with green dye.

Matrices were refrigerated for 24 hours followed by 24 hours of lyophilization and evaluated under excess moisture and ambient temperatures. The dissolution medium was not changed throughout the study. The results are plotted as an average of triplicate measurements. 


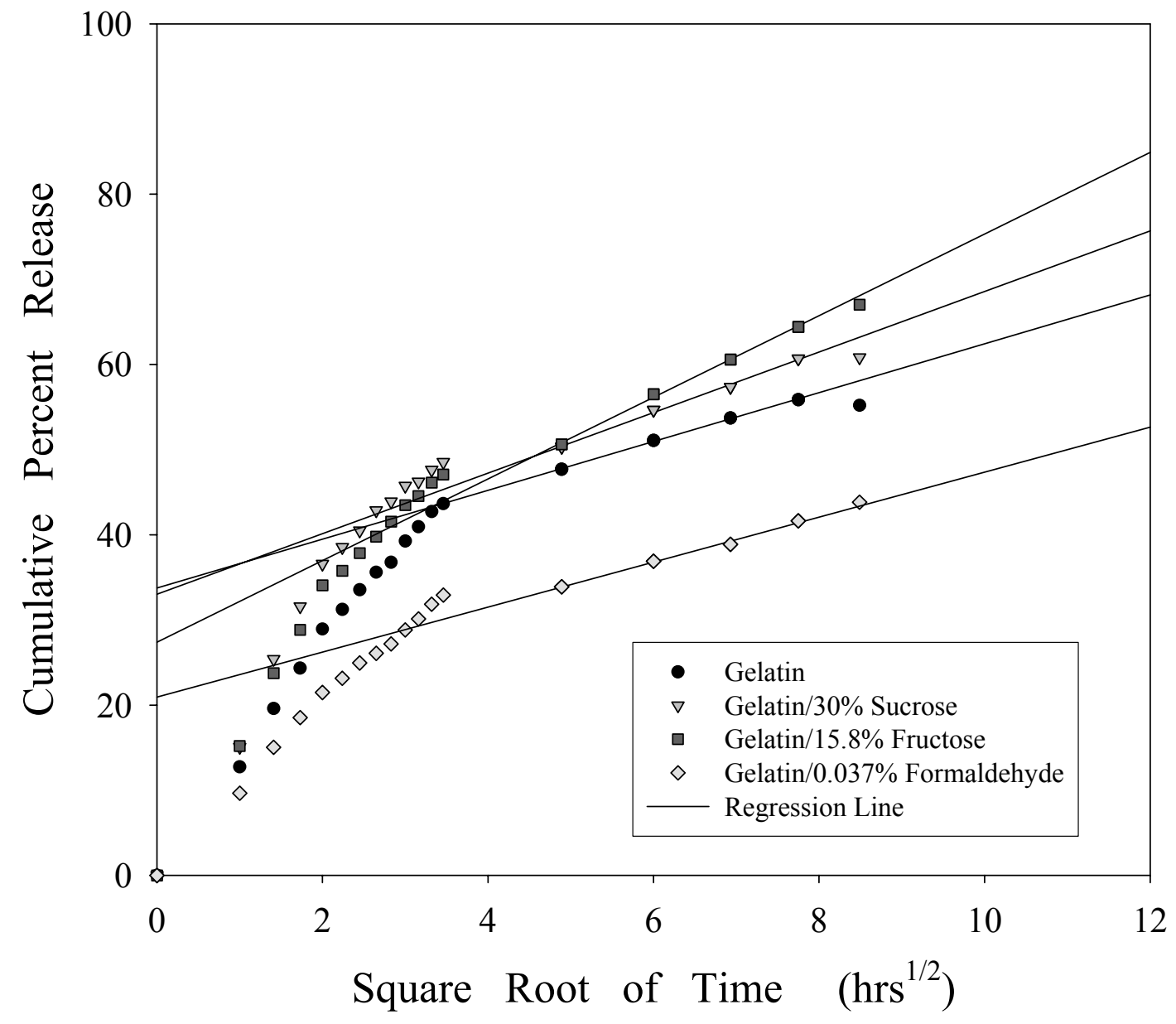

Figure 73. Linear regression for the Higuchi square root equation over the third release phase (Hours 24-64) for matrices loaded with green dye.

Matrices were refrigerated for 24 hours followed by 24 hours of lyophilization and evaluated under excess moisture and ambient temperatures. The dissolution medium was not changed throughout the study. The results are plotted as an average of triplicate measurements. 


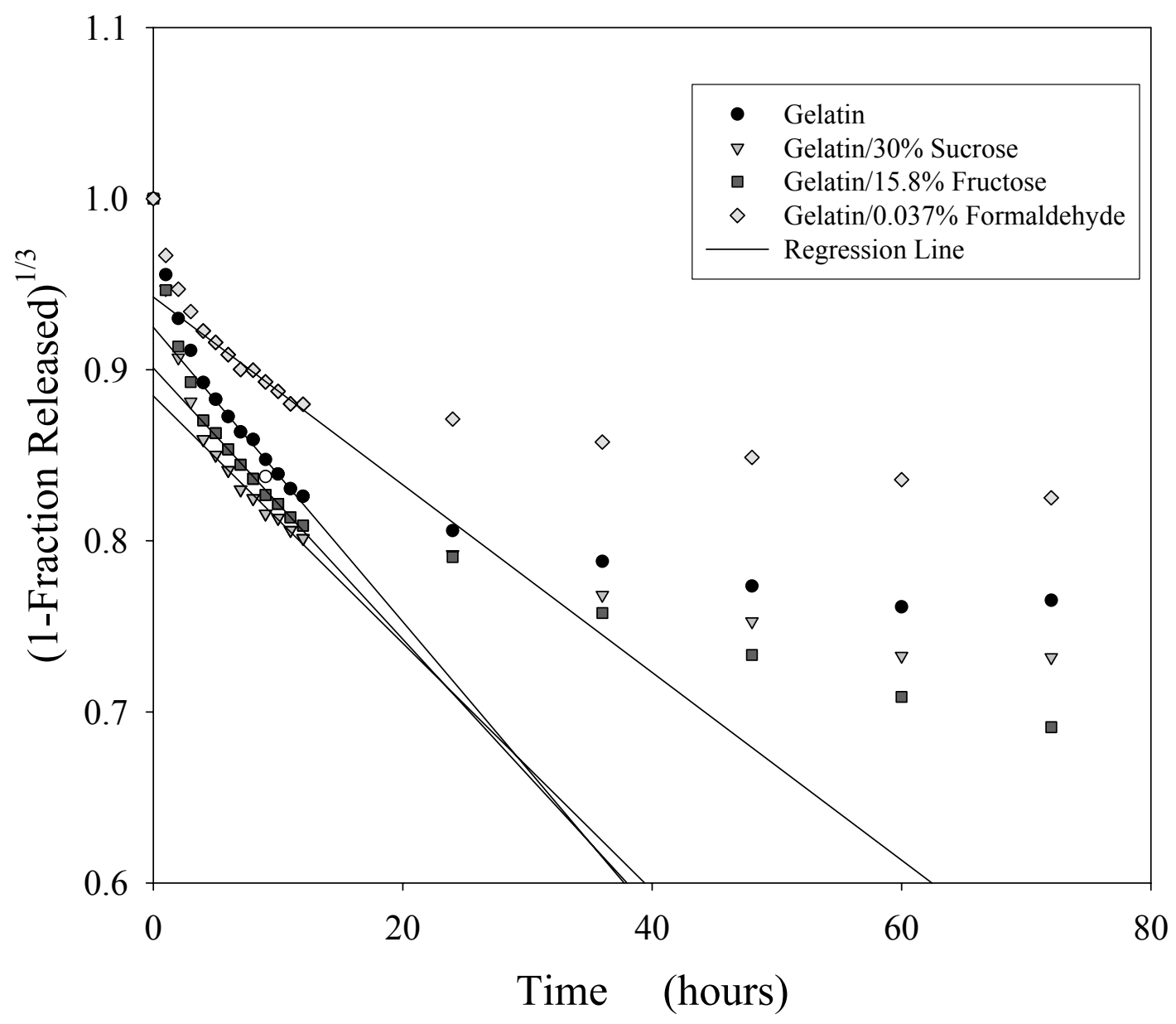

Figure 74. Linear regression for the Hixson-Crowell cube root equation over the second release phase (Hours 4-12) for matrices loaded with green dye.

Matrices were refrigerated for 24 hours followed by 24 hours of lyophilization and evaluated under excess moisture and ambient temperatures. The dissolution medium was not changed throughout the study. The results are plotted as an average of triplicate measurements. 


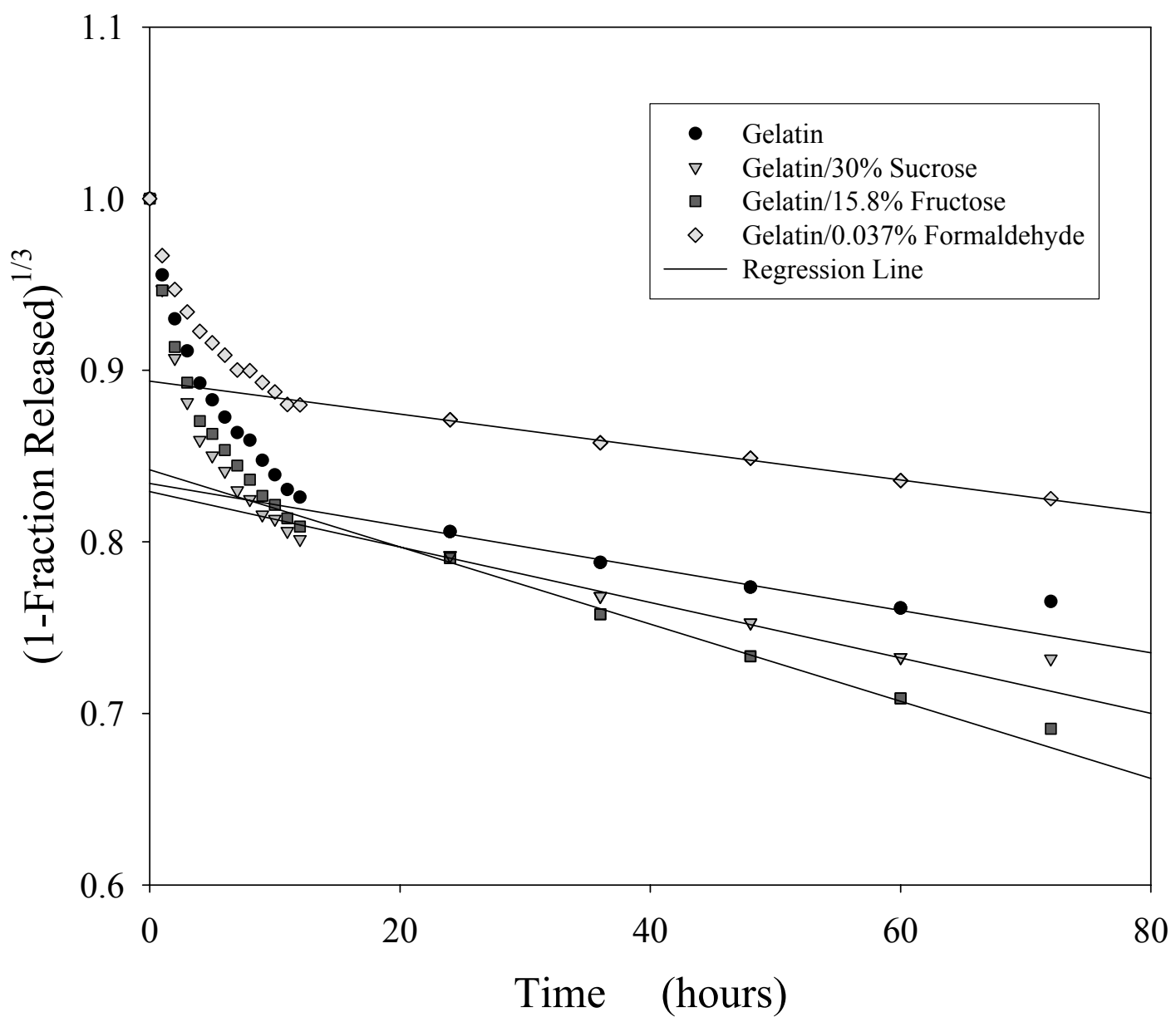

Figure 75. Linear regression for the Hixson-Crowell cube root equation over the third release phase (Hours 24-64) for matrices loaded with green dye.

Matrices were refrigerated for 24 hours followed by 24 hours of lyophilization and evaluated under excess moisture and ambient temperatures. The dissolution medium was not changed throughout the study. The results are plotted as an average of triplicate measurements. 


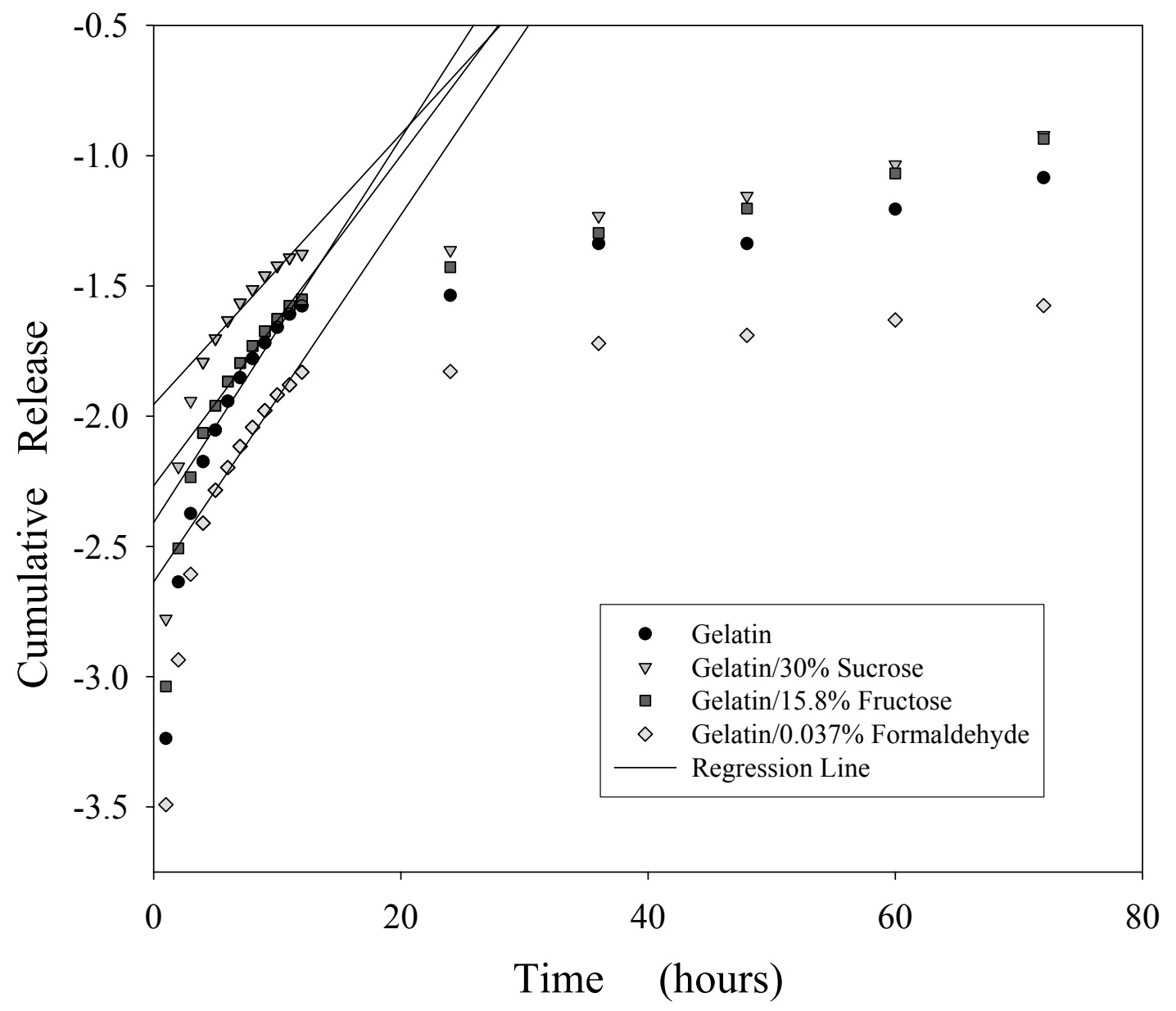

Figure 76. Linear regression for the first order equation over the second release phase (Hours 4-12) for matrices loaded with red dye.

Matrices were refrigerated for 24 hours followed by 24 hours of lyophilization and evaluated under excess moisture and ambient temperatures. The dissolution medium was not changed throughout the study. The results are plotted as an average of triplicate measurements. 


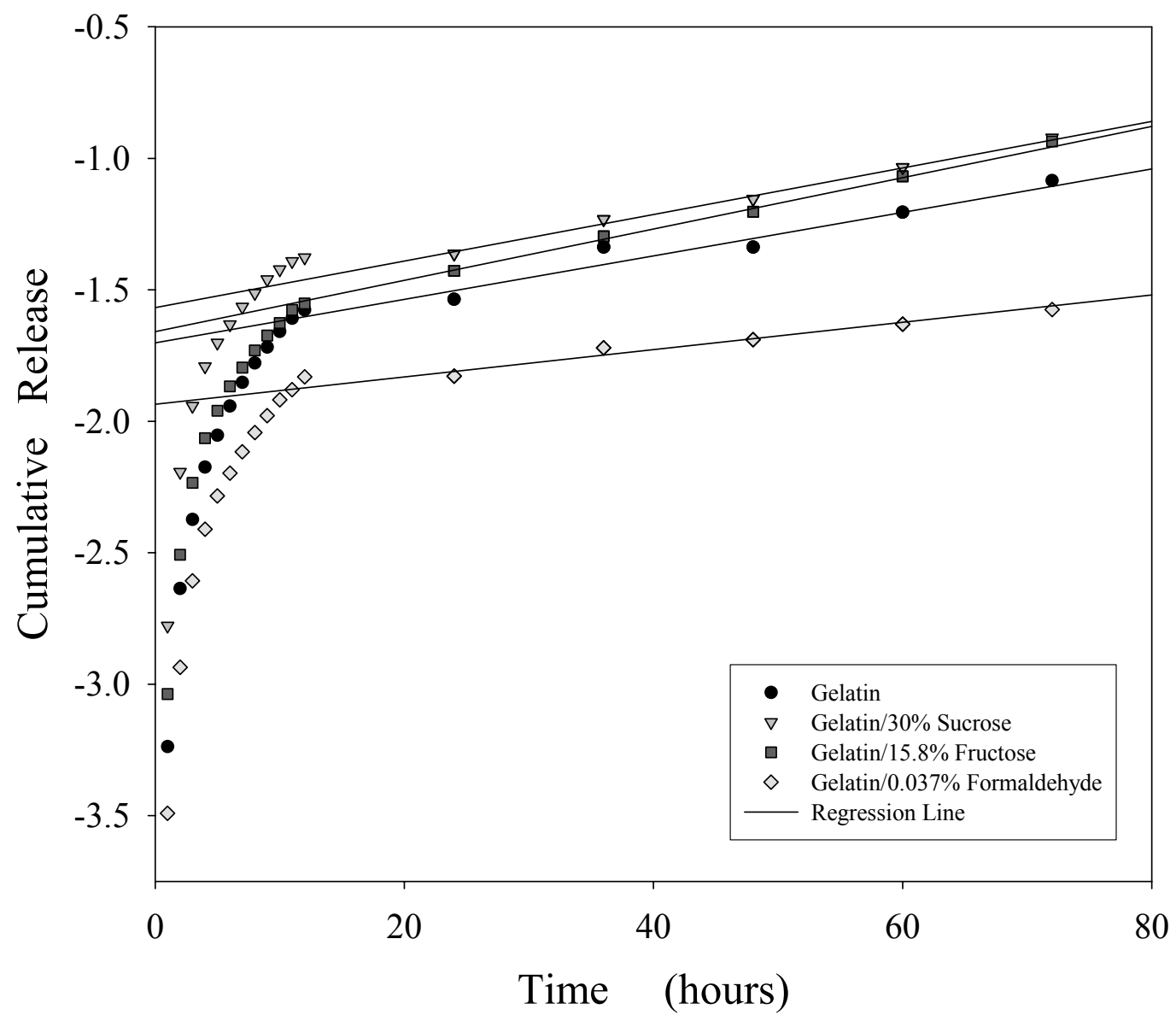

Figure 77. Linear regression for the first order equation over the third release phase (Hours 24-64) for matrices loaded with red dye.

Matrices were refrigerated for 24 hours followed by 24 hours of lyophilization and evaluated under excess moisture and ambient temperatures. The dissolution medium was not changed throughout the study. The results are plotted as an average of triplicate measurements. 


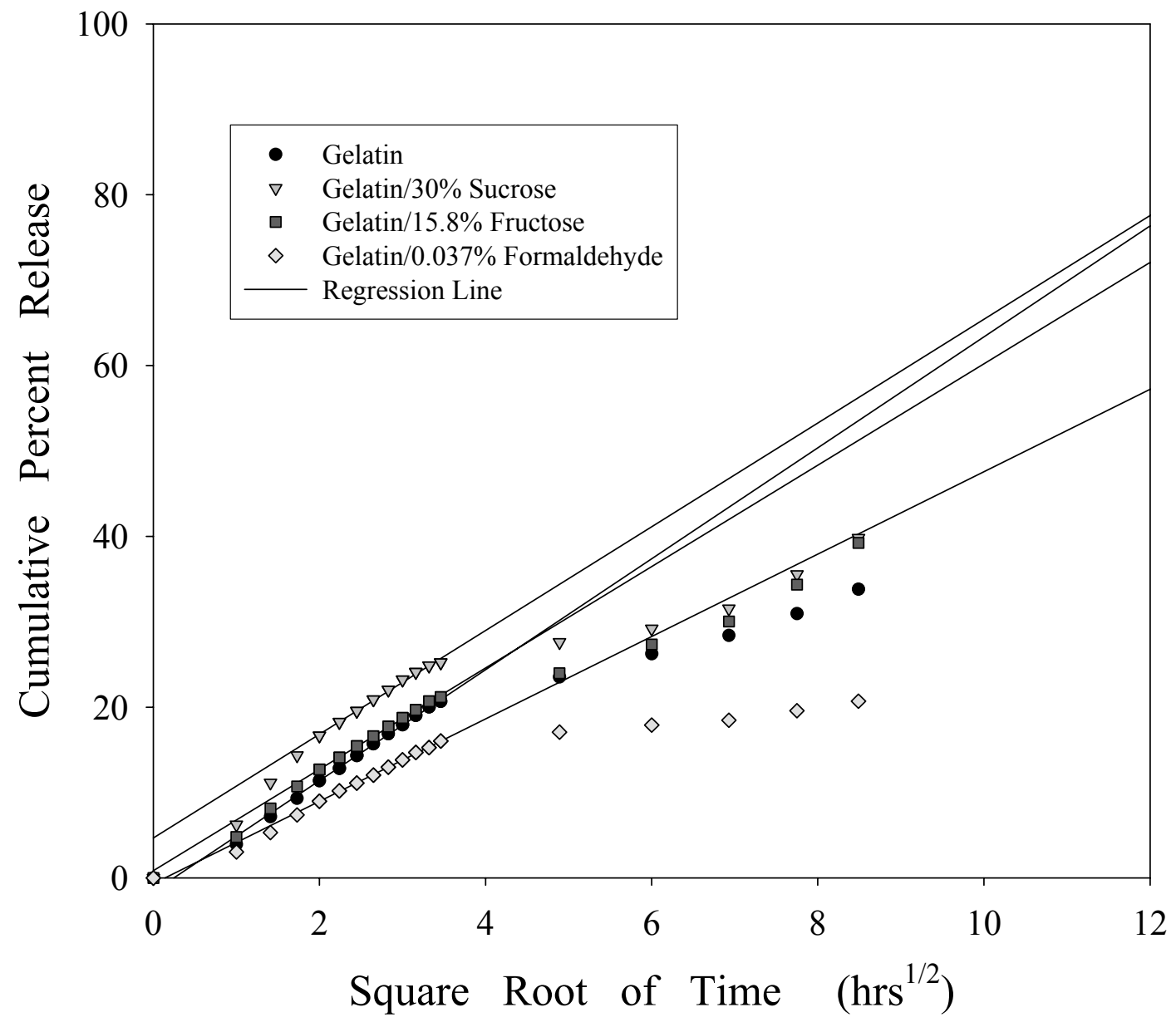

Figure 78. Linear regression for the Higuchi square root equation over the second release phase (Hours 4-12) for matrices loaded with red dye.

Matrices were refrigerated for 24 hours followed by 24 hours of lyophilization and evaluated under excess moisture and ambient temperatures. The dissolution medium was not changed throughout the study. The results are plotted as an average of triplicate measurements. 


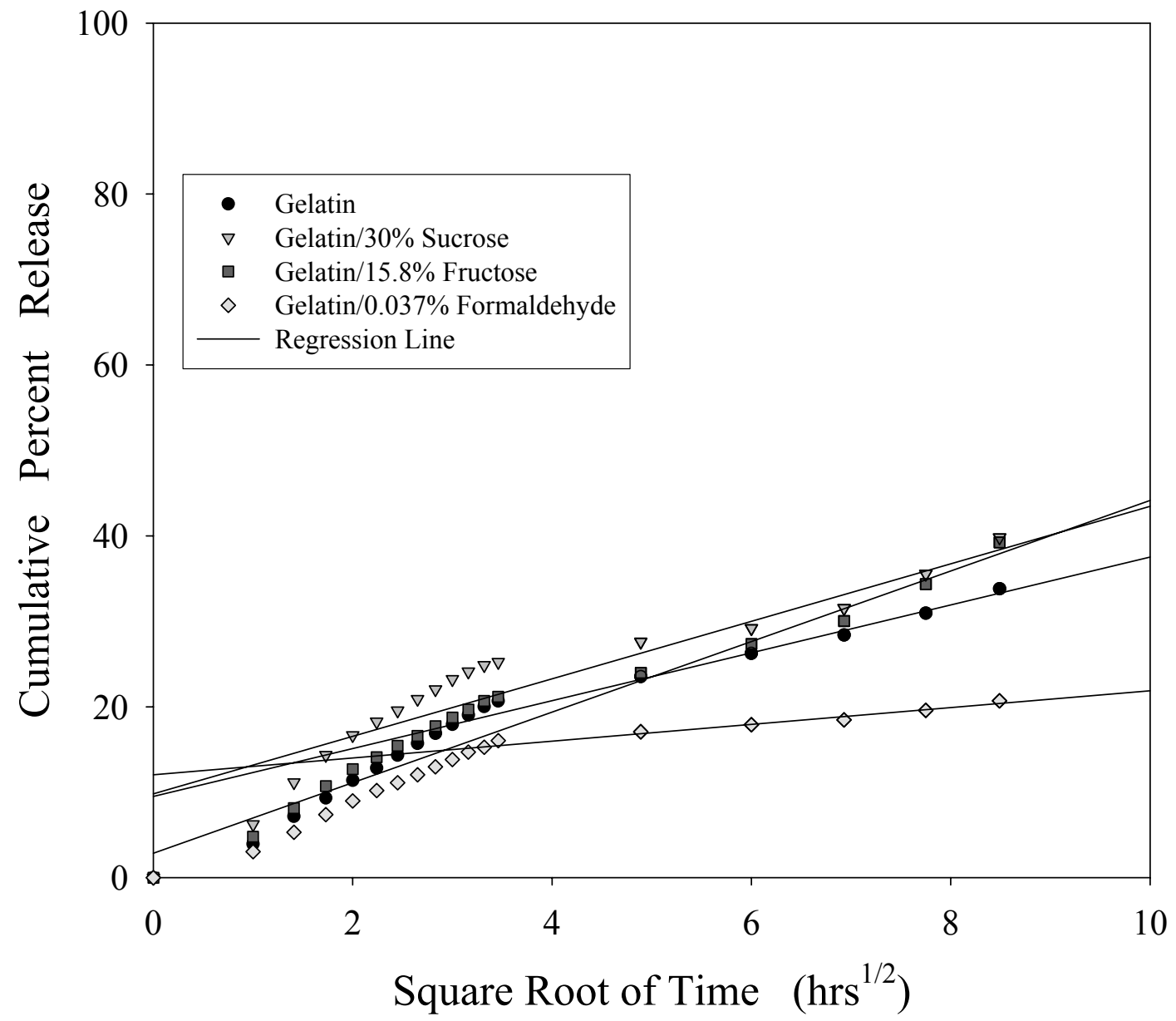

Figure 79. Linear regression for the Higuchi square root equation over the third release phase (Hours 24-64) for matrices loaded with red dye.

Matrices were refrigerated for 24 hours followed by 24 hours of lyophilization and evaluated under excess moisture and ambient temperatures. The dissolution medium was not changed throughout the study. The results are plotted as an average of triplicate measurements. 


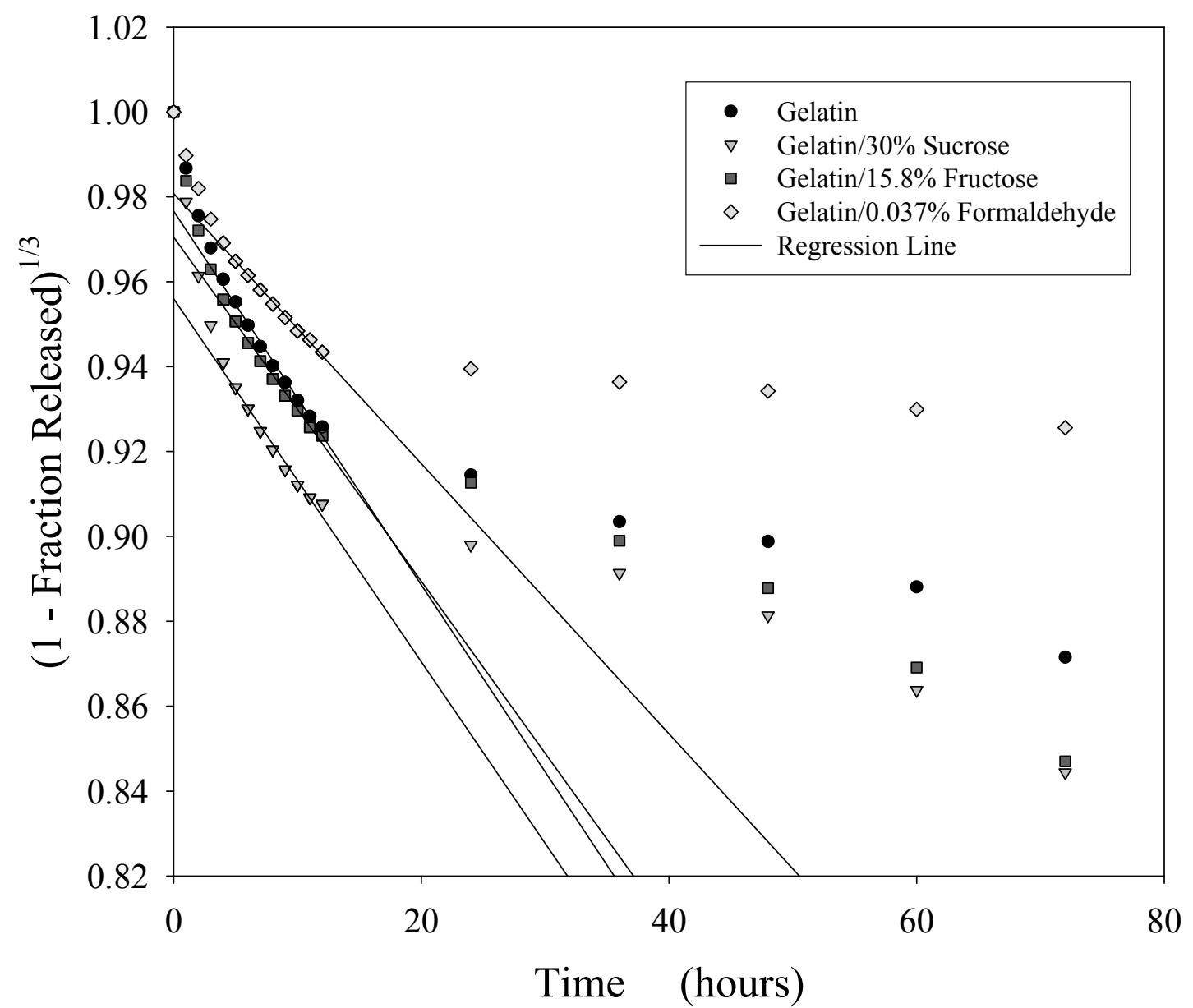

Figure 80. Linear regression for the Hixson-Crowell cube root equation over the second release phase (Hours 4-12) for matrices loaded with red dye.

Matrices were refrigerated for 24 hours followed by 24 hours of lyophilization and evaluated under excess moisture and ambient temperatures. The dissolution medium was not changed throughout the study. The results are plotted as an average of triplicate measurements. 


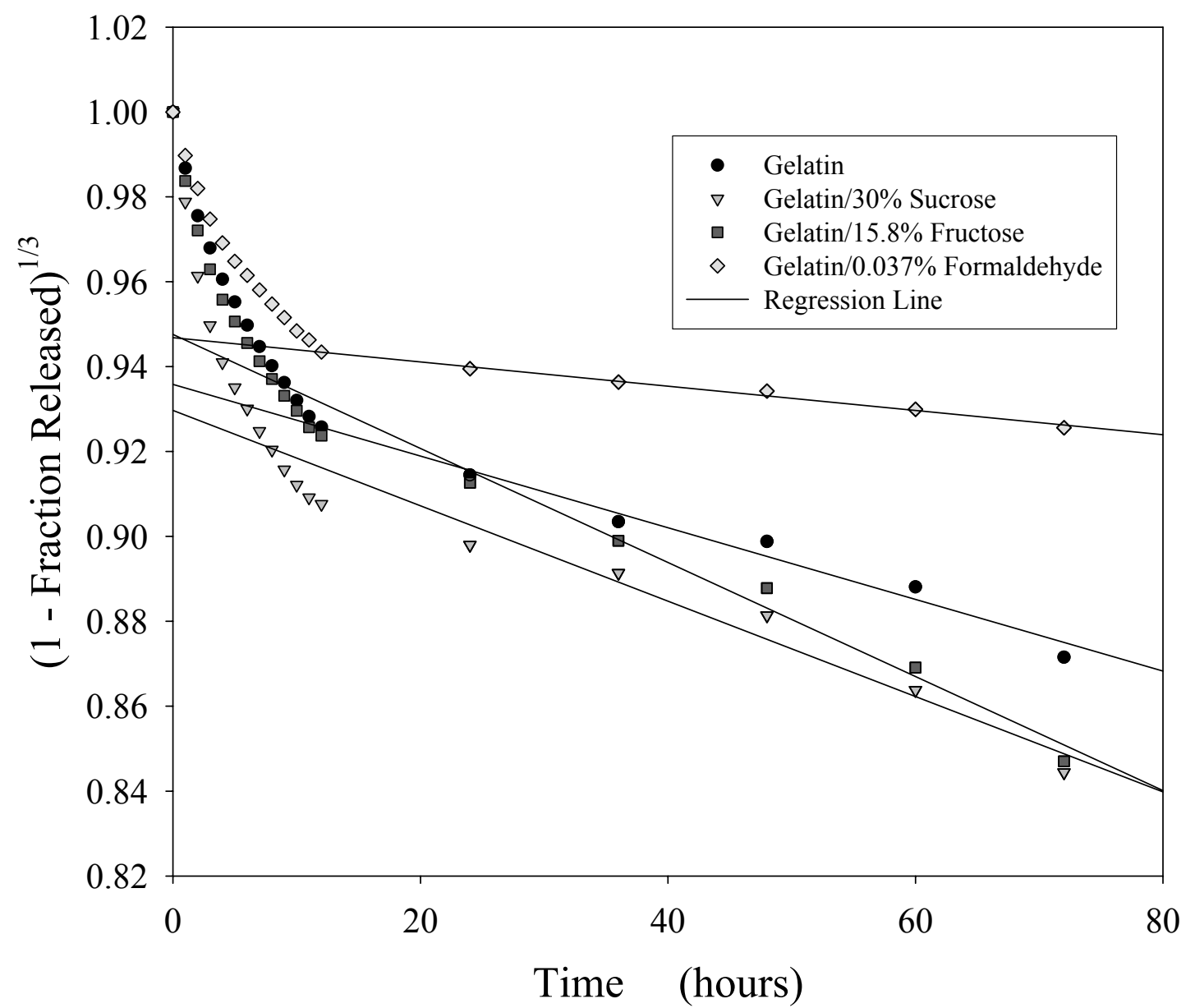

Figure 81. Linear regression for the Hixson-Crowell cube root equation over the third release phase (Hours 12-64) for matrices loaded with red dye.

Matrices were refrigerated for 24 hours followed by 24 hours of lyophilization and evaluated under excess moisture and ambient temperatures. The dissolution medium was not changed throughout the study. The results are plotted as an average of triplicate measurements. 
All phases of release correlate well with the Higuchi model (0.991) except the third release phase for the red dye. Upon comparison of the red and green formulations, the conditions which the matrices were prepared and evaluated were the same, indicating that the differences seen must be related to the physicochemical properties of the dyes. For the red, this may be due to an altered diffusion time and/or pathway required by the red components due to their charge and bulkiness. Tables 12 and 13 show the regression data for the Higuchi model. Slopes for the phases were determined from the fit of the Higuchi model. All four formulations demonstrate a decrease in the slopes as release progresses through the phases.

Further evidence confirming the relative validity of the Higuchi model was obtained by utilizing the differential form of the equation $[9,10]$. The differential forms of the Higuchi model is $d\left(M_{t} / M_{\infty}\right)=f\left(1 /\left(M_{t} / M_{\infty}\right)\right)$. The Higuchi model was the only model in which the differential forms were evaluated in this section. This is due to the fact that the Higuchi model describes the release of drug from a polymer matrix while the other two models describe the dissolution of the drug particles. The linearity was obtained when $d\left(M_{t} / M_{\infty}\right)$ was plotted as a function of $1 / M_{t} / M_{\infty}$ further confirming that the process follows the Higuchi model, as seen in Figures 82-89. All three phases of both dyes show linearity when evaluating the differential forms. This also confirms that the Higuchi model best describes the overall release of dye from the gelatin-based matrices. 
Table 12. Comparison of the regression data for three diffusional phases of the green dye.

Data represents a mean of 3 determinations. HCHO represents formaldehyde.

\begin{tabular}{|c|c|c|c|}
\hline GELATIN & Slope & Y-intercept & Correlation \\
\hline Hours 1-3 & 0.1590 & -0.0305 & 0.9989 \\
Hours 4-12 & 0.1030 & 0.0822 & 0.9971 \\
Hours 24-64 & 0.0269 & 0.3374 & 0.9989 \\
\hline
\end{tabular}

\begin{tabular}{|c|c|c|c|}
\hline $\begin{array}{c}\text { GELATIN/ } \\
\text { SUCROSE }\end{array}$ & Slope & Y-intercept & Correlation \\
\hline Hours 1-3 & 0.2275 & -0.0738 & 0.9947 \\
Hours 4-12 & 0.0828 & 0.2027 & 0.9912 \\
Hours 24-64 & 0.0355 & 0.3303 & 0.9965 \\
\hline
\end{tabular}

\begin{tabular}{|c|c|c|c|}
\hline $\begin{array}{c}\text { GELATIN/ } \\
\text { FRUCTOSE }\end{array}$ & Slope & Y-intercept & Correlation \\
\hline Hours 1-3 & 0.1877 & -0.0331 & 0.9944 \\
Hours 4-12 & 0.925 & 0.1533 & 0.9976 \\
Hours 24-64 & 0.4794 & 0.2738 & 0.9983 \\
\hline
\end{tabular}

\begin{tabular}{|c|c|c|c|}
\hline $\begin{array}{c}\text { GELATIN/ } \\
\text { HCHO }\end{array}$ & Slope & Y-intercept & Correlation \\
\hline Hours 1-3 & 0.1221 & -0.0245 & 0.9970 \\
Hours 4-12 & 0.0780 & 0.0564 & 0.9944 \\
Hours 24-64 & 0.0264 & 0.2093 & 0.9937 \\
\hline
\end{tabular}


Table 13. Comparison of the regression data for three diffusional phases of the red dye.

Data represents a mean of 3 determinations. HCHO represents formaldehyde.

\begin{tabular}{|c|c|c|c|}
\hline GELATIN & Slope & Y-intercept & Correlation \\
\hline Hours 1-3 & 0.0739 & -0.0340 & 0.9980 \\
Hours 4-11 & 0.0661 & -0.0188 & 0.9997 \\
Hours 12-64 & 0.0238 & 0.1215 & 0.9943 \\
\hline
\end{tabular}

\begin{tabular}{|c|c|c|c|}
\hline $\begin{array}{c}\text { GELATIN/ } \\
\text { SUCROSE }\end{array}$ & Slope & Y-intercept & Correlation \\
\hline Hours 1-3 & 0.1116 & -0.0484 & 0.9973 \\
Hours 4-11 & 0.0632 & 0.0409 & 0.9986 \\
Hours 12-64 & 0.0225 & 0.1673 & 0.9326 \\
\hline
\end{tabular}

\begin{tabular}{|c|c|c|c|}
\hline $\begin{array}{c}\text { GELATIN/ } \\
\text { FRUCTOSE }\end{array}$ & Slope & Y-intercept & Correlation \\
\hline Hours 1-3 & 0.0810 & -0.0329 & 1.0000 \\
Hours 4-11 & 0.0605 & 0.0059 & 0.9999 \\
Hours 12-64 & 0.0299 & 0.1001 & 0.9684 \\
\hline
\end{tabular}

\begin{tabular}{|c|c|c|c|}
\hline $\begin{array}{c}\text { GELATIN/ } \\
\text { HCHO }\end{array}$ & Slope & Y-intercept & Correlation \\
\hline Hours 1-3 & 0.0591 & -0.0291 & 0.9979 \\
Hours 4-11 & 0.0482 & -0.0067 & 0.9991 \\
Hours 12-64 & 0.0079 & 0.1320 & 0.9837 \\
\hline
\end{tabular}




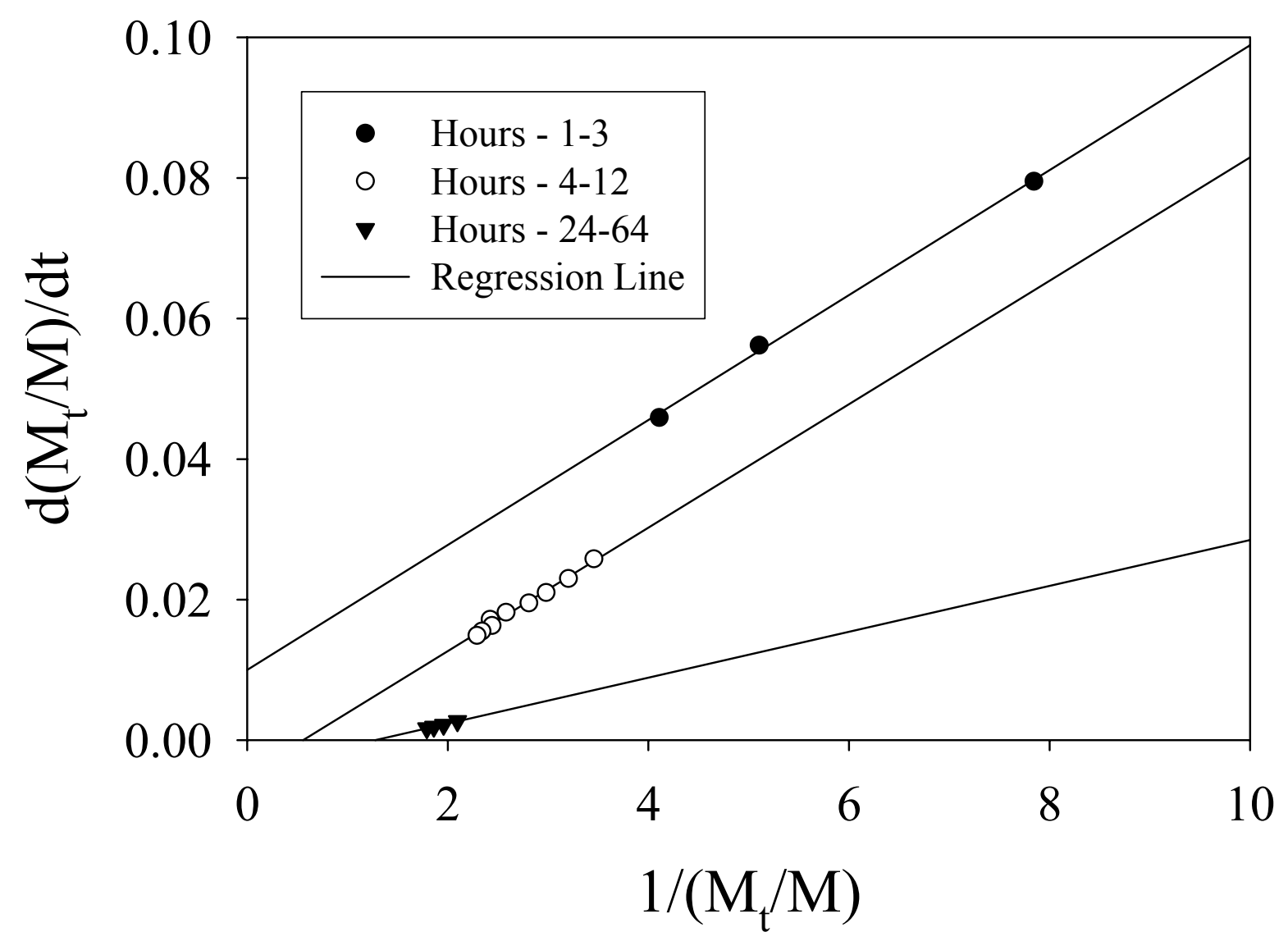

Figure 82. Plot of $d\left(M_{t} / M_{\infty}\right)$ versus $1 /\left(M_{t} / M_{\infty}\right)$ for the gelatin matrices loaded with green dye.

Matrices were refrigerated for 24 hours followed by 24 hours of lyophilization and evaluated under excess moisture and ambient temperatures. The dissolution medium was not changed throughout the study. The results are plotted as an average of triplicate measurements. 


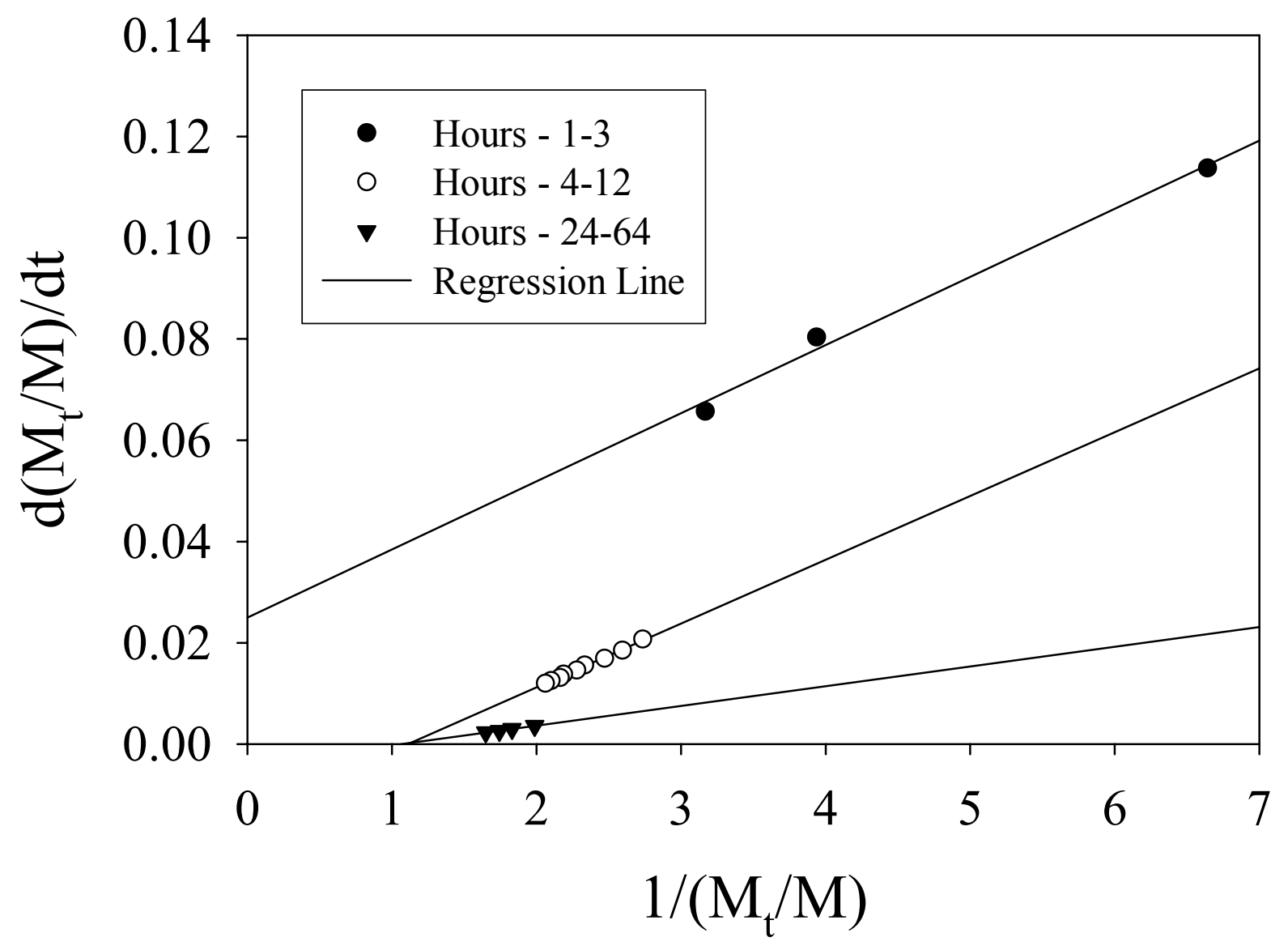

Figure 83. Plot of $d\left(M_{t} / M_{\infty}\right)$ versus $1 /\left(M_{t} / M_{\infty}\right)$ for the gelatin/30\% sucrose matrices loaded with green dye.

Matrices were refrigerated for 24 hours followed by 24 hours of lyophilization and evaluated under excess moisture and ambient temperatures. The dissolution medium was not changed throughout the study. The results are plotted as an average of triplicate measurements. 


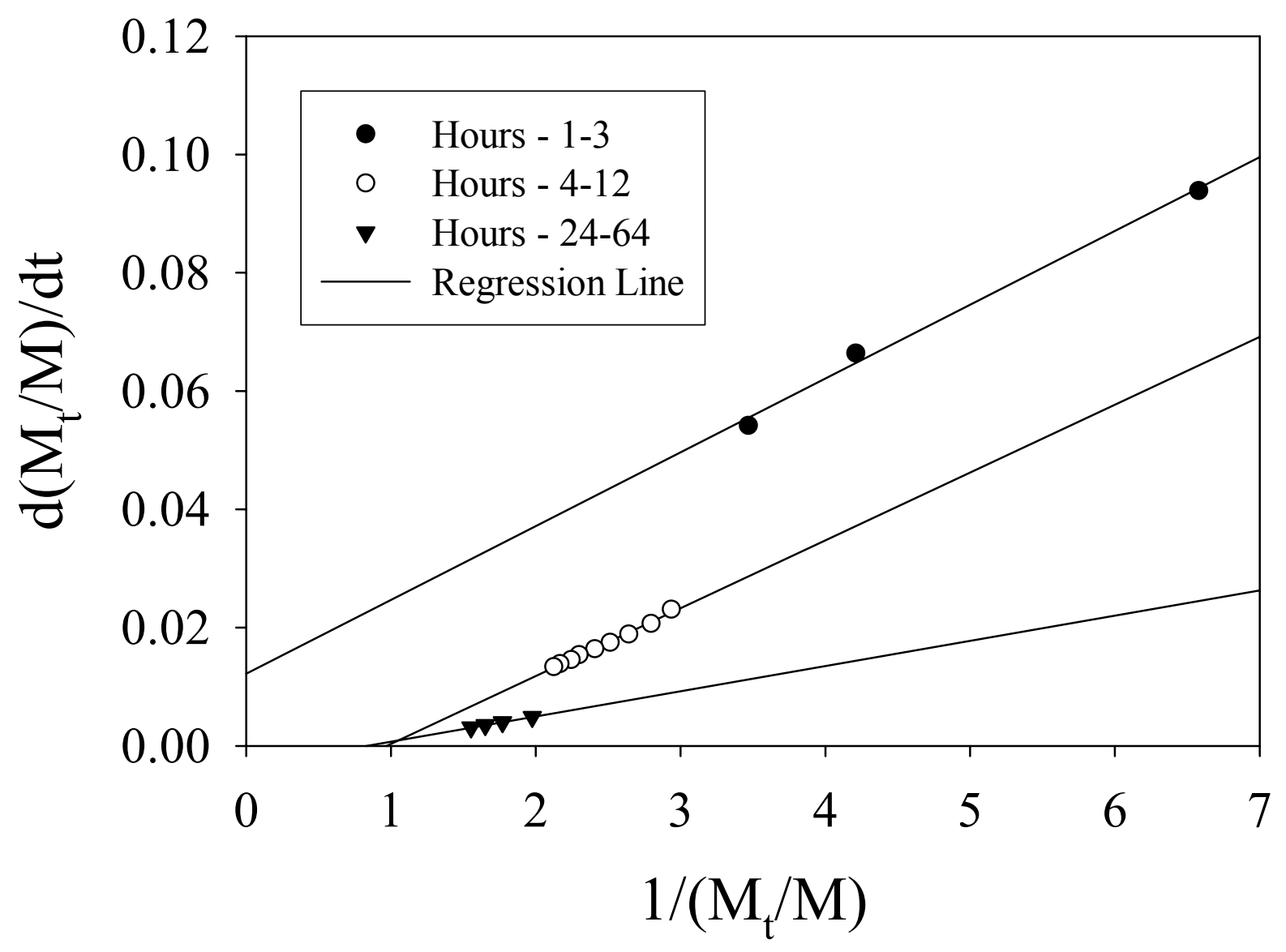

Figure 84. Plot of $d\left(M_{t} / M_{\infty}\right)$ versus $1 /\left(M_{t} / M_{\infty}\right)$ for the gelatin/15.8\% fructose matrices loaded with green dye.

Matrices were refrigerated for 24 hours followed by 24 hours of lyophilization and evaluated under excess moisture and ambient temperatures. The dissolution medium was not changed throughout the study. The results are plotted as an average of triplicate measurements. 


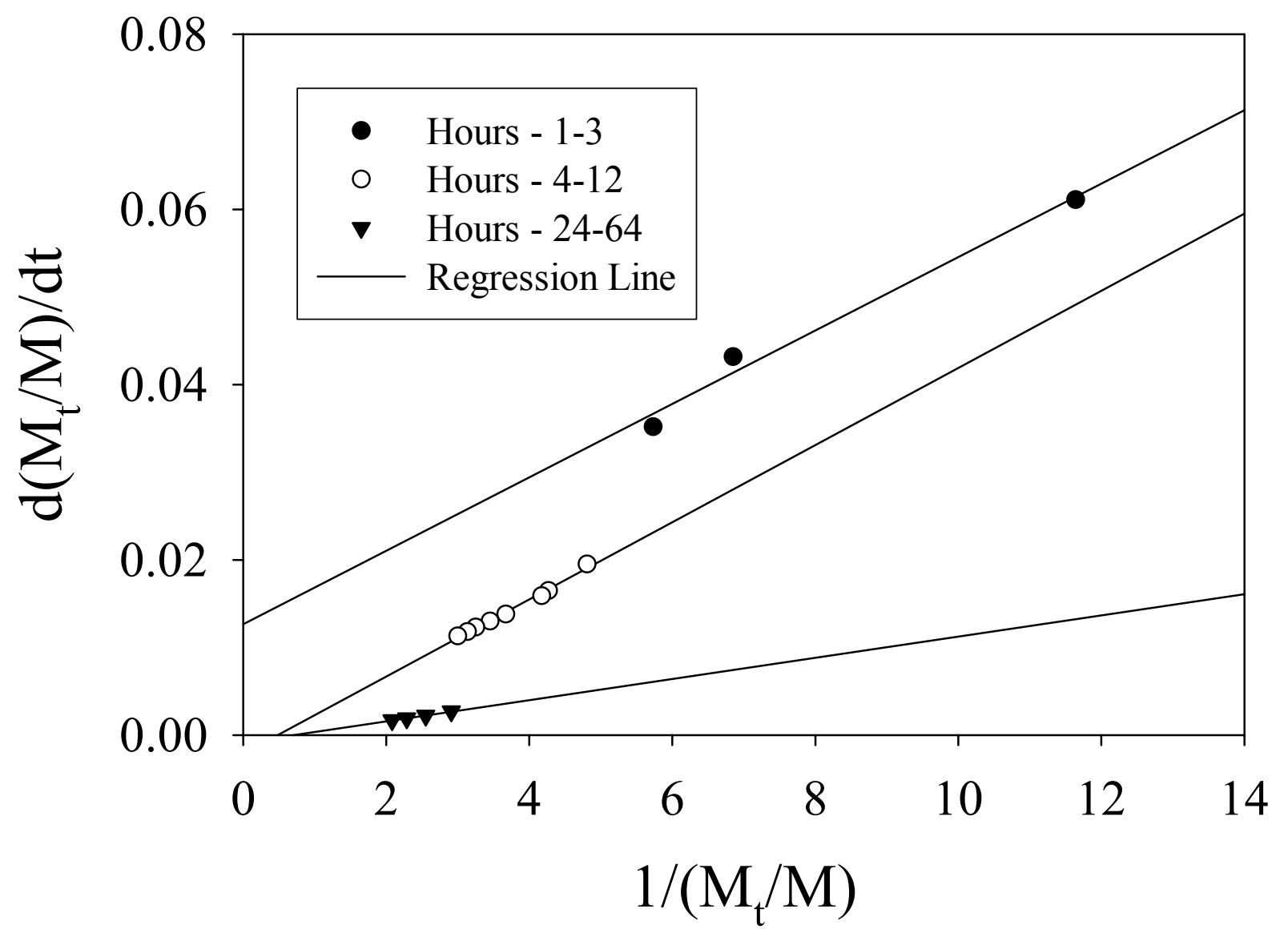

Figure 85. Plot of $d\left(M_{t} / M_{\infty}\right)$ versus $1 /\left(M_{t} / M_{\infty}\right)$ for the gelatin/0.037\% formaldehyde matrices loaded with green dye.

Matrices were refrigerated for 24 hours followed by 24 hours of lyophilization and evaluated under excess moisture and ambient temperatures. The dissolution medium was not changed throughout the study. The results are plotted as an average of triplicate measurements. 


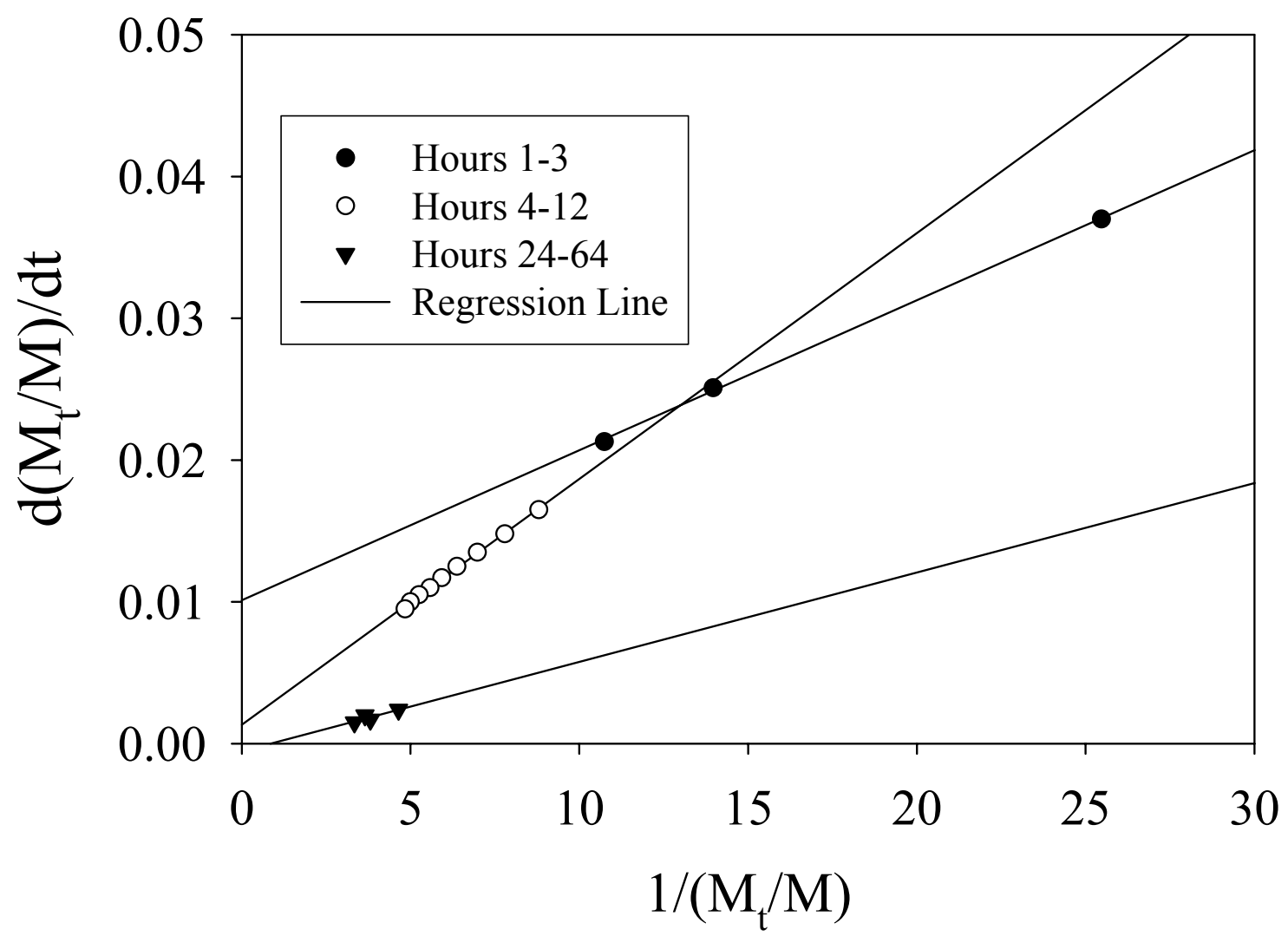

Figure 86. Plot of $d\left(M_{t} / M_{\infty}\right)$ versus $1 /\left(M_{t} / M_{\infty}\right)$ for the gelatin matrices loaded with red dye.

Matrices were refrigerated for 24 hours followed by 24 hours of lyophilization and evaluated under excess moisture and ambient temperatures. The dissolution medium was not changed throughout the study. The results are plotted as an average of triplicate measurements. 


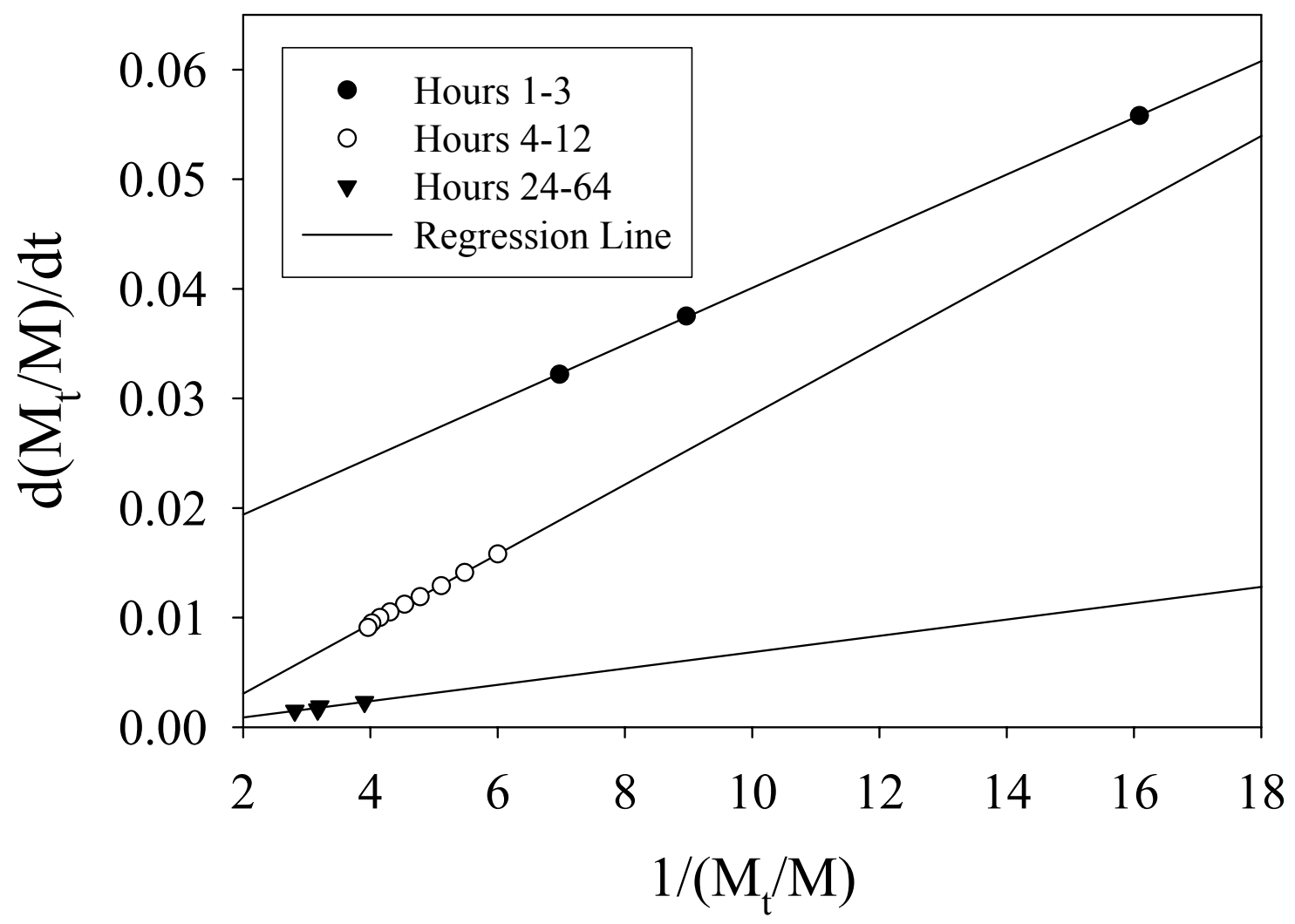

Figure 87. Plot of $d\left(M_{t} / M_{\infty}\right)$ versus $1 /\left(M_{t} / M_{\infty}\right)$ for the gelatin/30\% sucrose matrices loaded with red dye.

Matrices were refrigerated for 24 hours followed by 24 hours of lyophilization and evaluated under excess moisture and ambient temperatures. The dissolution medium was not changed throughout the study. The results are plotted as an average of triplicate measurements. 


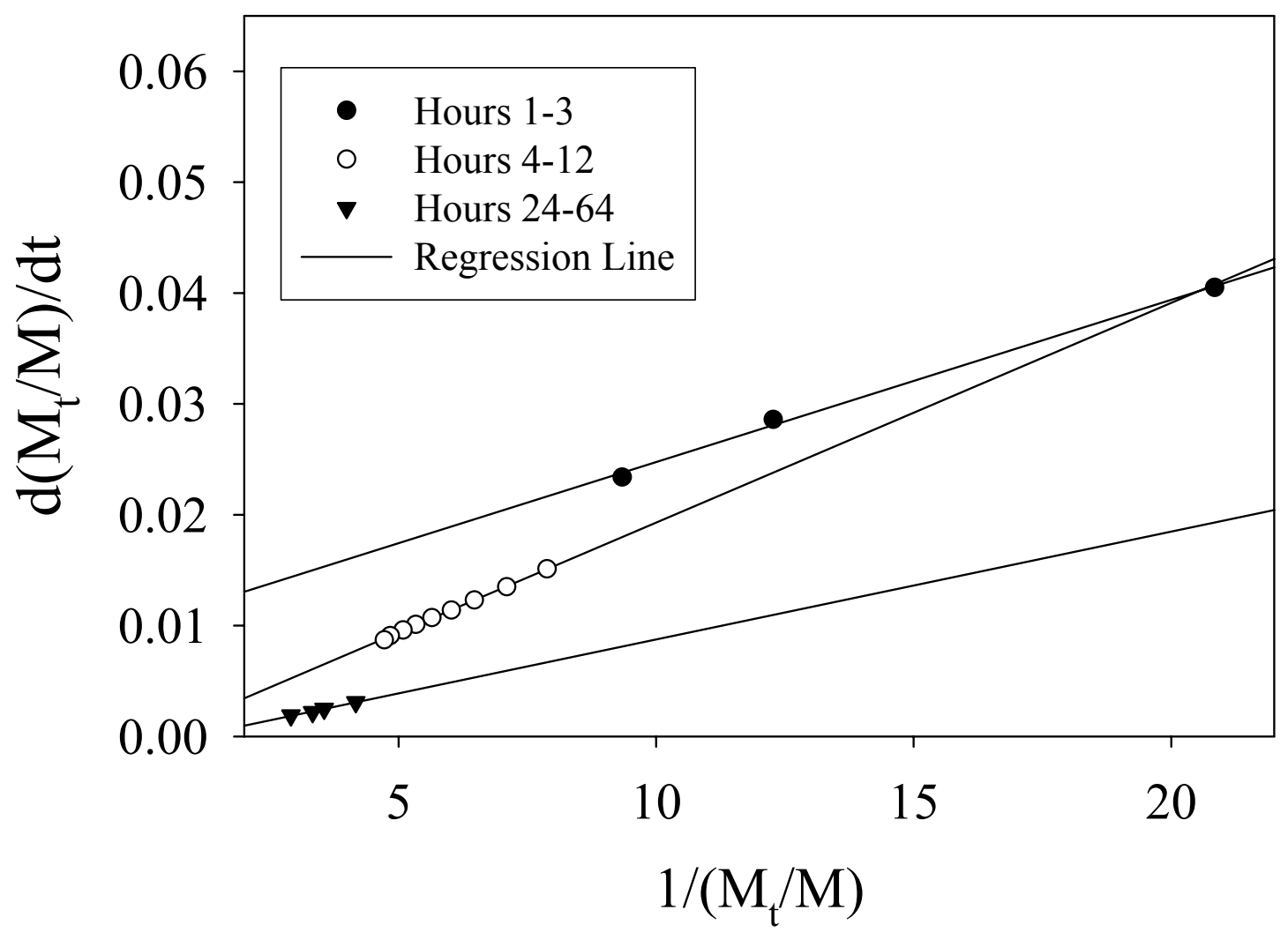

Figure 88. Plot of $d\left(M_{t} / M_{\infty}\right)$ versus $1 /\left(M_{t} / M_{\infty}\right)$ for the gelatin/15.8\% fructose matrices loaded with red dye.

Matrices were refrigerated for 24 hours followed by 24 hours of lyophilization and evaluated under excess moisture and ambient temperatures. The dissolution medium was not changed throughout the study. The results are plotted as an average of triplicate measurements. 


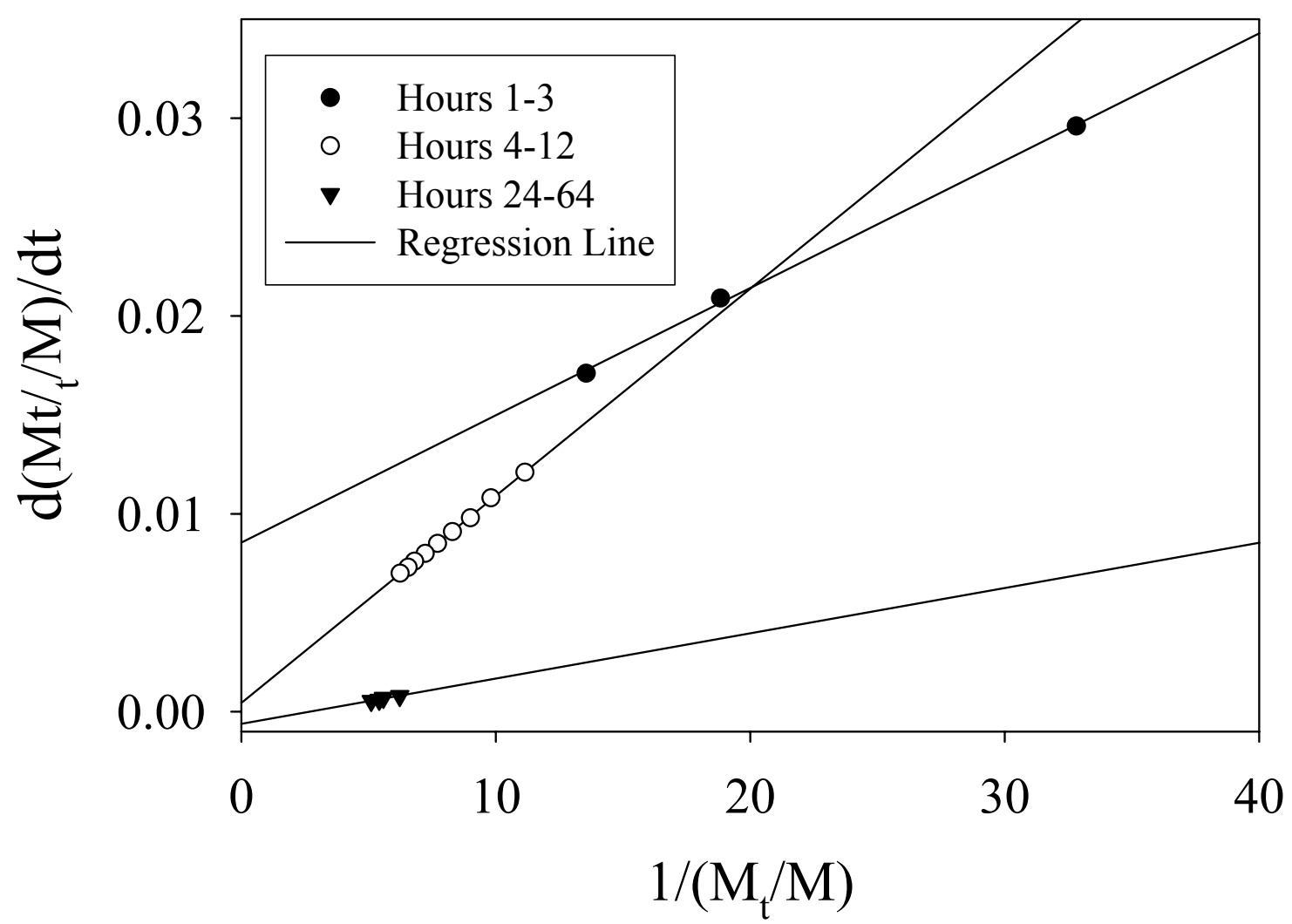

Figure 89. Plot of $d\left(M_{t} / M_{\infty}\right)$ versus $1 /\left(M_{t} / M_{\infty}\right)$ for the gelatin/ $\mathbf{0 . 0 3 7 \%}$ formaldehyde matrices loaded with red dye.

Matrices were refrigerated for 24 hours followed by 24 hours of lyophilization and evaluated under excess moisture and ambient temperatures. The dissolution medium was not changed throughout the study. The results are plotted as an average of triplicate measurements. 


\section{Sink Conditions}

Since the initial dye studies resulted in a build up of dye in the bulk, likely influencing the third phase of release, and dye remaining in the matrix, a second set of experiments were designed to assess the release characteristics of matrices while maintaining sink (more dilute) conditions. This release data (Figures 90 and 91) demonstrates a biphasic pattern of release similar to the more concentrated conditions presented in the beginning of this chapter (Figures 68 and 69). An initial rate, relating to the initial swelling phase seen when the matrix is first exposed to the water, was seen within the first 4-8 hours. This is likely due to solvent convective effects, as well as the free dye on the matrix surface. For the dye associated with the gelatin or within the matrix pores, a diffusion process becomes the main driving force, resulting in a second phase occurring between 12-32 hours for green loaded and 16-24 hours for red loaded matrices. Notably, the third phases of these profiles show an increase in percent dye release when compared with those of concentrated (non-sink) bulk conditions.

The data was first examined by fitting it to the three equations presented at the beginning of this chapter. The correlation coefficients indicate that the best fit is obtained with the Higuchi diffusional model rather than the first order or Hixson-Crowell cube root equation, as would be expected. Tables 14 and 15 suggest that dye release follows a simple diffusional mechanism. The three models demonstrated good correlations, ranging from 0.94 to 1.00 . However, based on the three models presented in the introduction of this chapter, the Higuchi model best predicts the release of dye from the non-cross-linked gelatin, gelatin/sucrose, gelatin/fructose, and gelatin/formaldehyde matrices (Figures 93-105). The first order release model and the Hixson-Crowell 


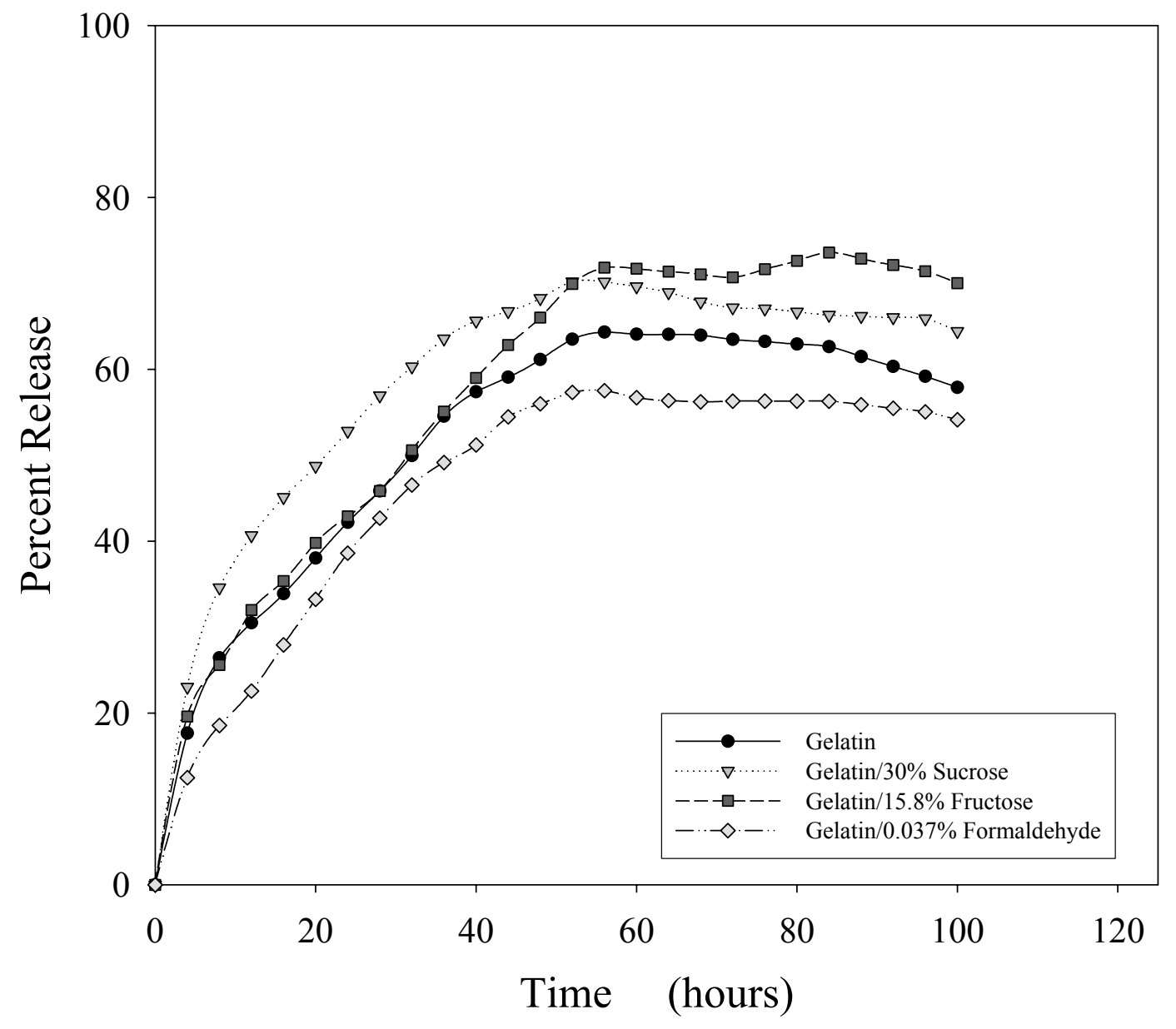

Figure 90. Release profile of gelatin, gelatin/30\% sucrose, gelatin/15.8\% fructose, and gelatin $\mathbf{0 . 0 3 7 \%}$ formaldehyde matrices loaded with green dye.

Matrices were refrigerated for 24 hours followed by 24 hours of lyophilization and evaluated under excess moisture, ambient temperatures, and sink conditions. The results are plotted as an average of triplicate measurements. 


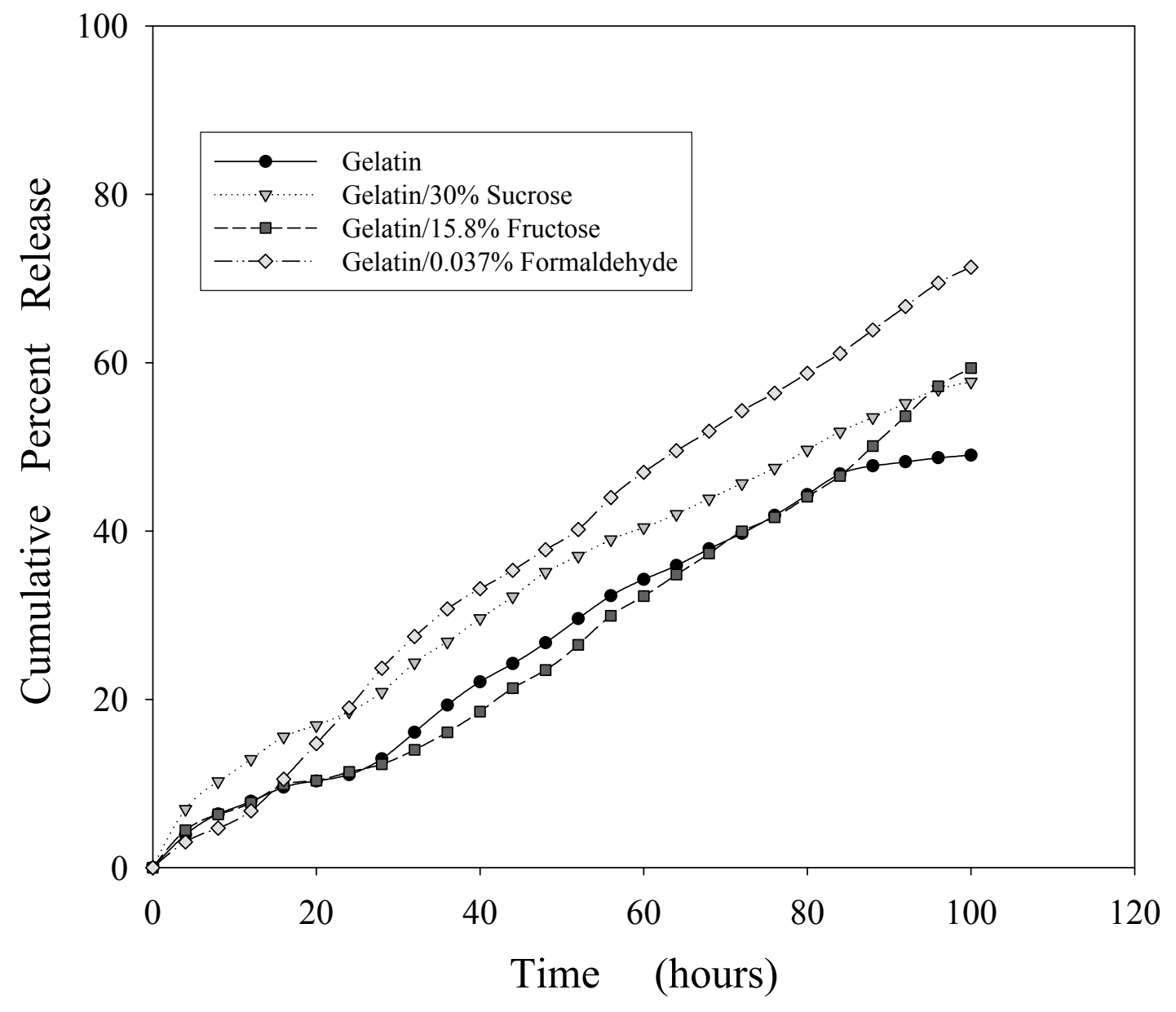

Figure 91. Release profile of gelatin, gelatin/30\% sucrose, gelatin $/ \mathbf{1 5 . 8 \%}$ fructose, and gelatin $/ \mathbf{0 . 0 3 7 \%}$ formaldehyde matrices loaded with red dye.

Matrices were refrigerated for 24 hours followed by 24 hours of lyophilization and evaluated under excess moisture, ambient temperatures, and sink conditions. The results are plotted as an average of triplicate measurements. 
Table 14. Correlation coefficients of different mathematical models for green dye.

Data represents a mean of 3 determinations. HCHO represents formaldehyde.

\begin{tabular}{|l|ccc|}
\hline GELATIN & $\begin{array}{c}\text { First Order } \\
\text { Model }\end{array}$ & $\begin{array}{c}\text { Higuchi Square Root } \\
\text { of Time Model }\end{array}$ & $\begin{array}{c}\text { Hixson-Crowell Cube Root } \\
\text { Equation }\end{array}$ \\
\hline Hours 12-32 & 0.9960 & 0.9924 & 0.9976 \\
Hours 36-52 & 0.9923 & 0.9946 & 0.9952 \\
\hline
\end{tabular}

\begin{tabular}{|c|c|c|c|}
\hline $\begin{array}{c}\text { GELATIN/ } \\
\text { SUCROSE }\end{array}$ & $\begin{array}{c}\text { First Order } \\
\text { Model }\end{array}$ & $\begin{array}{c}\text { Higuchi Square Root } \\
\text { of Time Model }\end{array}$ & $\begin{array}{c}\text { Hixson-Crowell Cube Root } \\
\text { Equation }\end{array}$ \\
\hline Hours 12-32 & 0.9931 & 0.9970 & 0.9991 \\
Hours 36-52 & 0.9915 & 0.9902 & 0.9911 \\
\hline
\end{tabular}

\begin{tabular}{|c|c|c|c|}
\hline $\begin{array}{c}\text { GELATIN/ } \\
\text { FRUCTOSE }\end{array}$ & $\begin{array}{c}\text { First Order } \\
\text { Model }\end{array}$ & $\begin{array}{c}\text { Higuchi Square Root } \\
\text { of Time Model }\end{array}$ & $\begin{array}{c}\text { Hixson-Crowell Cube Root } \\
\text { Equation }\end{array}$ \\
\hline Hours 12-32 & 0.9920 & 0.9904 & 0.9772 \\
Hours 36-52 & 0.9966 & 0.9989 & 0.9802 \\
\hline
\end{tabular}

\begin{tabular}{|c|c|c|c|}
\hline $\begin{array}{c}\text { GELATIN/ } \\
\text { HCHO }\end{array}$ & $\begin{array}{c}\text { First Order } \\
\text { Model }\end{array}$ & $\begin{array}{c}\text { Higuchi Square Root } \\
\text { of Time Model }\end{array}$ & $\begin{array}{c}\text { Hixson-Crowell Cube Root } \\
\text { Equation }\end{array}$ \\
\hline Hours 12-32 & 0.9722 & 0.9990 & 0.9983 \\
Hours 36-52 & 0.9844 & 0.9928 & 0.9925 \\
\hline
\end{tabular}


Table 15. Correlation coefficients of different mathematical models for red dye.

Data represents a mean of 3 determinations. HCHO represents formaldehyde.

\begin{tabular}{|c|ccc|}
\hline GELATIN & $\begin{array}{c}\text { First Order } \\
\text { Model }\end{array}$ & $\begin{array}{c}\text { Higuchi Square Root } \\
\text { of Time Model }\end{array}$ & $\begin{array}{c}\text { Hixson-Crowell Cube Root } \\
\text { Equation }\end{array}$ \\
\hline Hours 4-12 & 0.9494 & 0.9960 & 0.9824 \\
Hours 16-24 & 0.9999 & 0.9986 & 0.9995 \\
& & 0.9981 & 0.9965 \\
\hline
\end{tabular}

\begin{tabular}{|c|ccc|}
\hline $\begin{array}{c}\text { GELATIN/ } \\
\text { SUCROSE }\end{array}$ & $\begin{array}{c}\text { First Order } \\
\text { Model }\end{array}$ & $\begin{array}{c}\text { Higuchi Square Root } \\
\text { of Time Model }\end{array}$ & $\begin{array}{c}\text { Hixson-Crowell Cube Root } \\
\text { Equation }\end{array}$ \\
\hline Hours 4-12 & 0.9783 & 0.9998 & 0.9968 \\
Hours 16-24 & 0.9994 & 0.9943 & 0.9974 \\
& & 0.9956 & 0.9915 \\
\hline
\end{tabular}

\begin{tabular}{|c|c|c|c|}
\hline $\begin{array}{c}\text { GELATIN/ } \\
\text { FRUCTOSE }\end{array}$ & $\begin{array}{c}\text { First Order } \\
\text { Model }\end{array}$ & $\begin{array}{c}\text { Higuchi Square Root } \\
\text { of Time Model }\end{array}$ & $\begin{array}{c}\text { Hixson-Crowell Cube Root } \\
\text { Equation }\end{array}$ \\
\hline Hours 4-12 & 0.9747 & 1.0000 & 0.9918 \\
Hours 16-24 & 0.9624 & 0.9982 & 0.9503 \\
& & 0.9938 & 0.9887 \\
Hours 28-72 & 0.9844 & & \\
\hline
\end{tabular}

\begin{tabular}{|c|c|c|c|}
\hline $\begin{array}{c}\text { GELATIN/ } \\
\text { HCHO }\end{array}$ & $\begin{array}{c}\text { First Order } \\
\text { Model }\end{array}$ & $\begin{array}{c}\text { Higuchi Square Root } \\
\text { of Time Model }\end{array}$ & $\begin{array}{c}\text { Hixson-Crowell Cube Root } \\
\text { Equation }\end{array}$ \\
\hline Hours 4-12 & 0.9973 & 0.9806 & 0.9954 \\
Hours 16-24 & 0.9929 & 0.9994 & 0.9999 \\
& & 0.9944 & 0.9963 \\
Hours 28-72 & 0.9803 & & \\
\hline
\end{tabular}




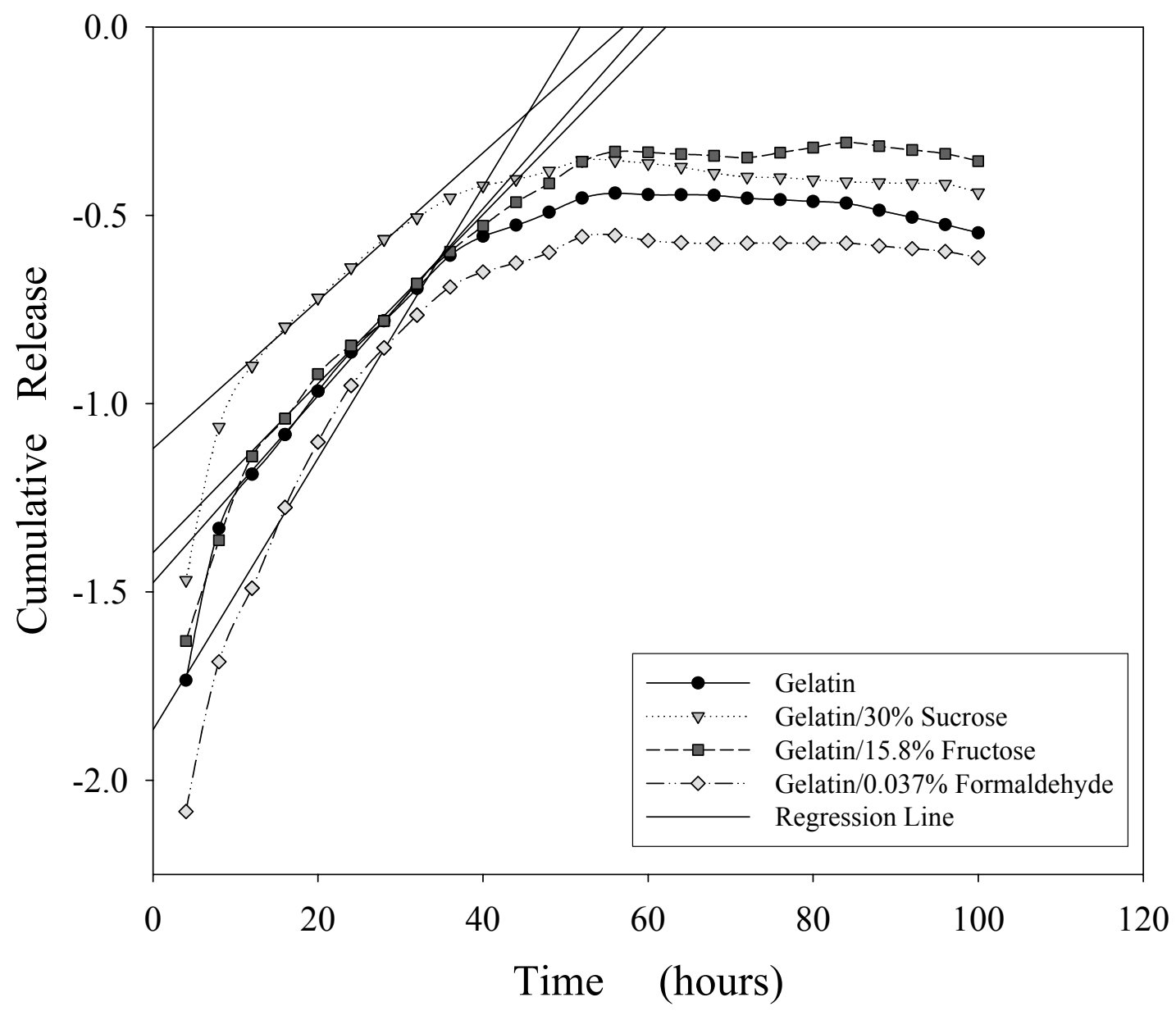

Figure 92. Linear regression for the first order equation over the second release phase (Hours 12-32) for matrices loaded with green dye.

Matrices were refrigerated for 24 hours followed by 24 hours of lyophilization and evaluated under excess moisture, ambient temperatures, and sink conditions. The results are plotted as an average of triplicate measurements. 


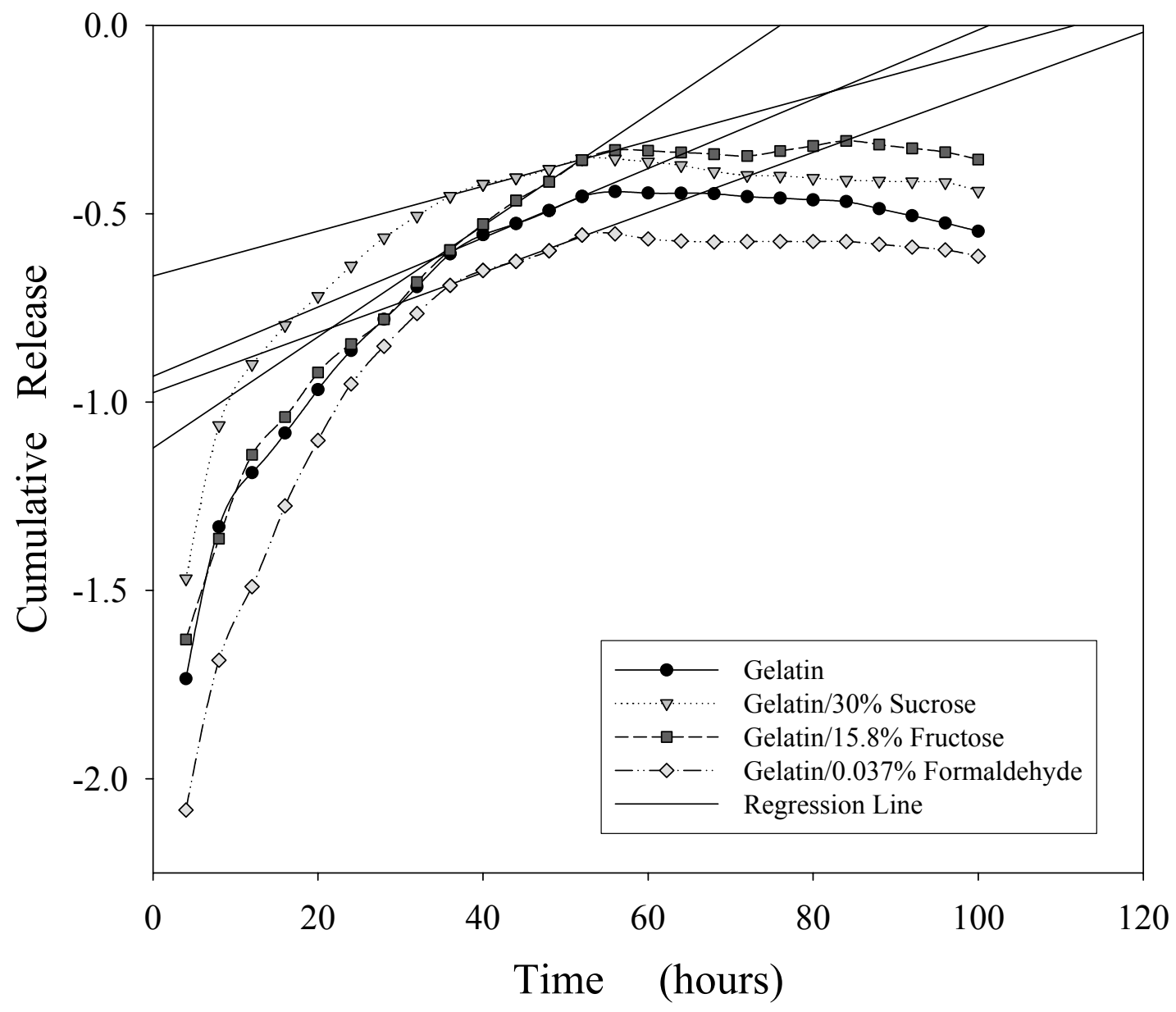

Figure 93. Linear regression for the first order equation over the third release phase (Hours 36-52) for matrices loaded with green dye.

Matrices were refrigerated for 24 hours followed by 24 hours of lyophilization and evaluated under excess moisture, ambient temperatures, and sink conditions. The results are plotted as an average of triplicate measurements. 


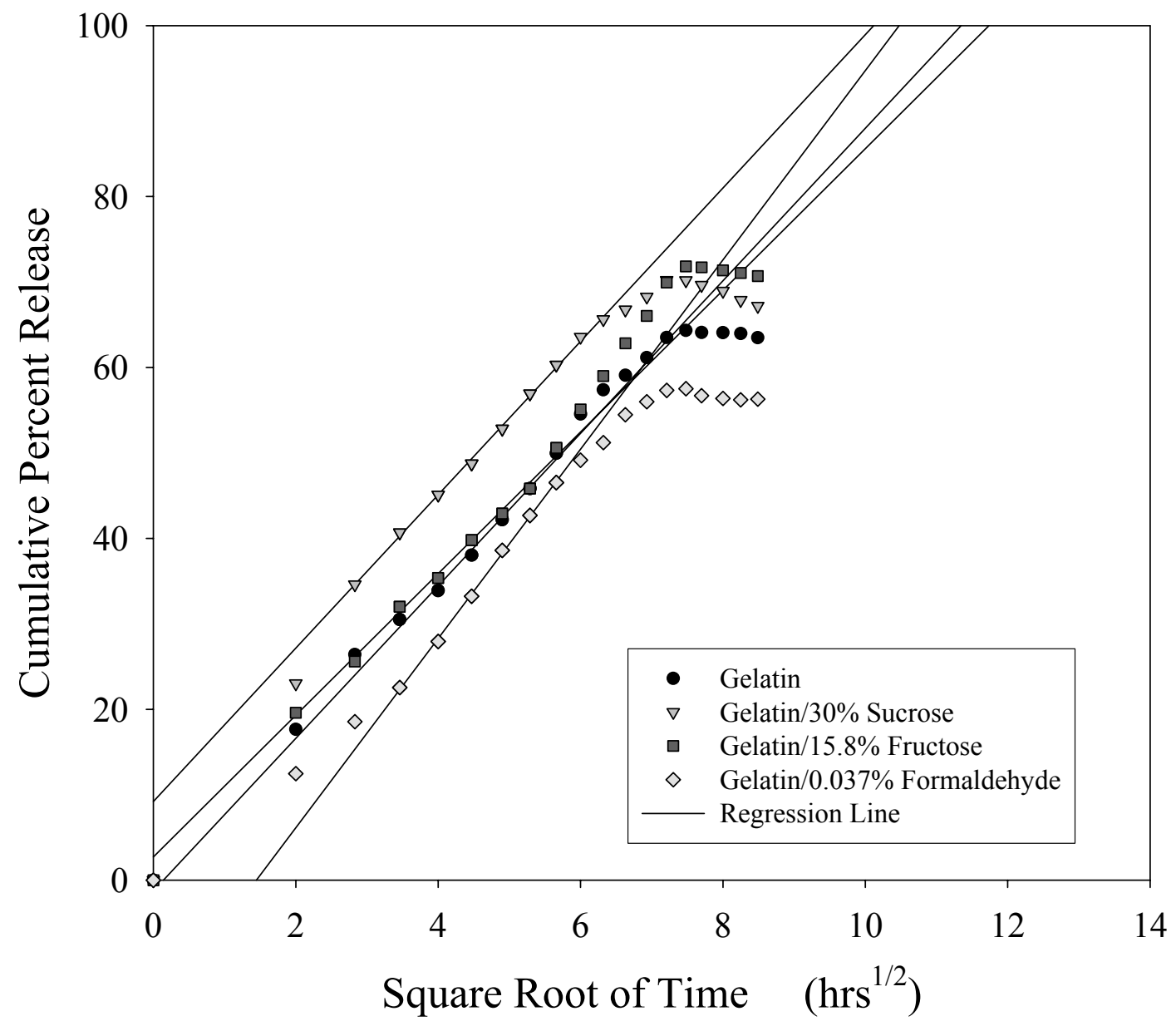

Figure 94. Linear regression for the Higuchi square root equation over the second release phase (Hours 12-32) for matrices loaded with green dye.

Matrices were refrigerated for 24 hours followed by 24 hours of lyophilization and evaluated under excess moisture, ambient temperatures, and sink conditions. The results are plotted as an average of triplicate measurements. 


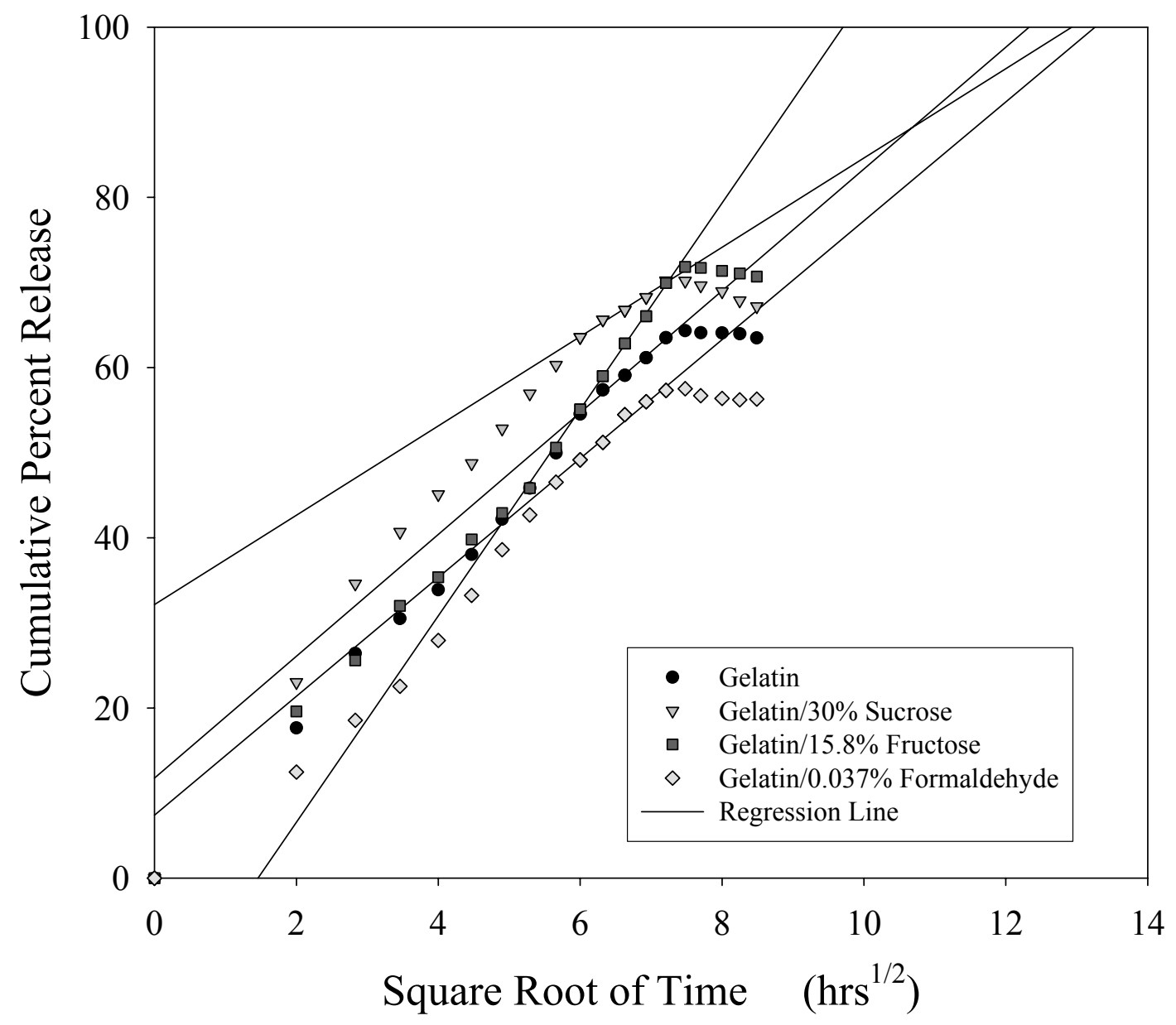

Figure 95. Linear regression for the Higuchi square root equation over the third release phase (Hours 36-52) for matrices loaded with green dye.

Matrices were refrigerated for 24 hours followed by 24 hours of lyophilization and evaluated under excess moisture, ambient temperatures, and sink conditions. The results are plotted as an average of triplicate measurements. 


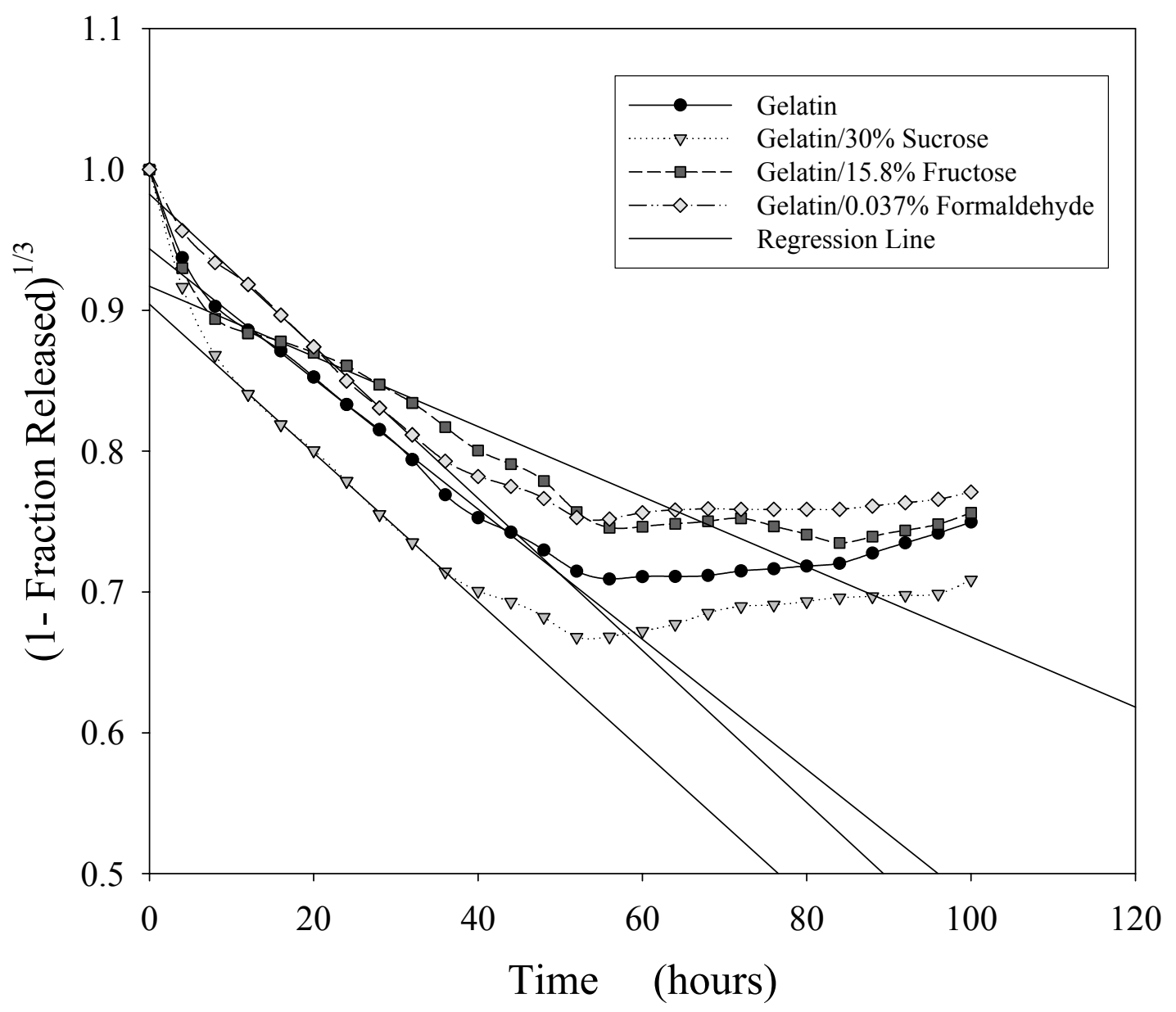

Figure 96. Linear regression for the Hixson-Crowell cube root equation over the second release phase (Hours 12-32) for matrices loaded with green dye.

Matrices were refrigerated for 24 hours followed by 24 hours of lyophilization and evaluated under excess moisture, ambient temperatures, and sink conditions. The results are plotted as an average of triplicate measurements. 


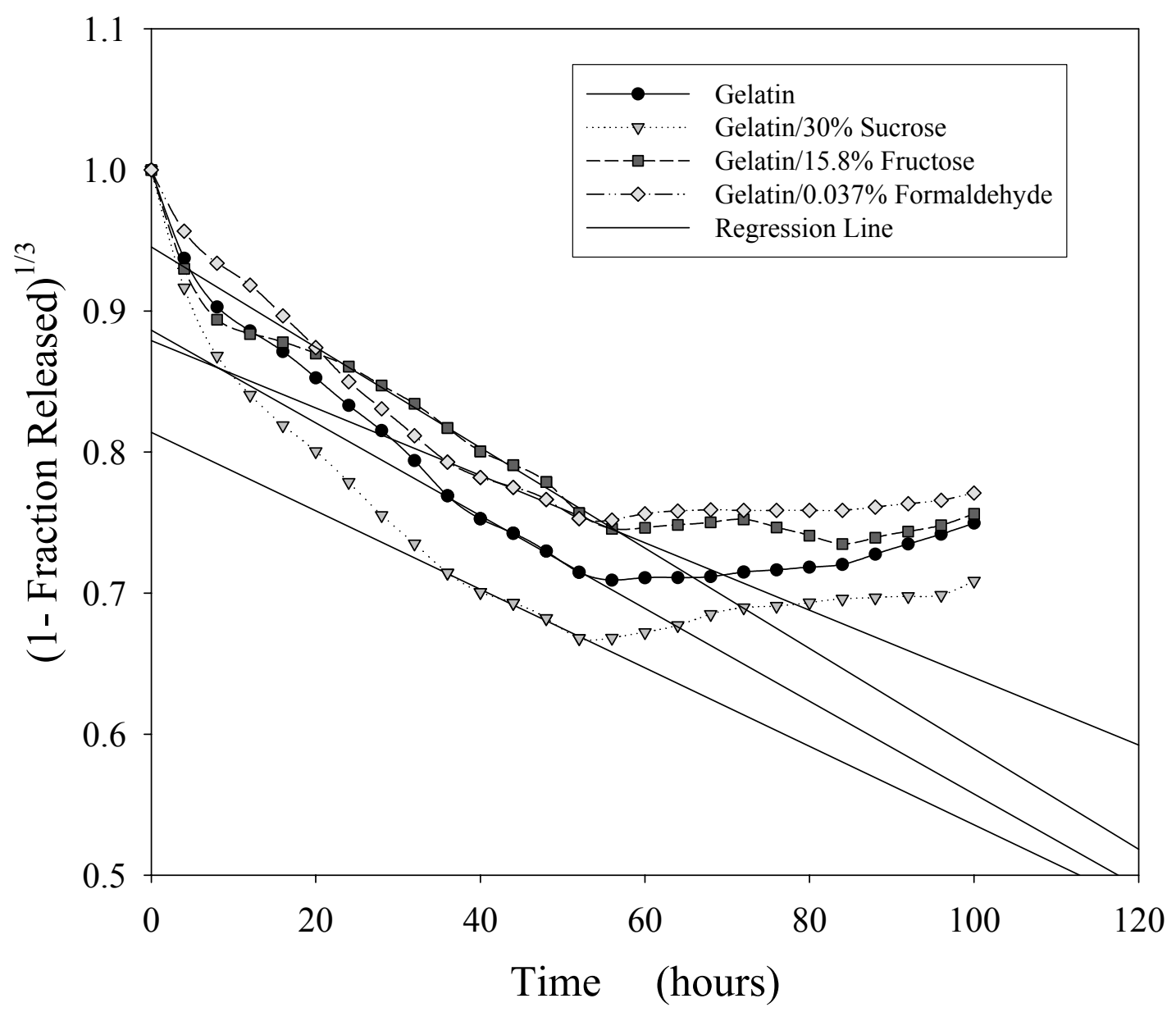

Figure 97. Linear regression for the Hixson-Crowell cube root equation over the third release phase (Hours 36-52) for matrices loaded with green dye.

Matrices were refrigerated for 24 hours followed by 24 hours of lyophilization and evaluated under excess moisture, ambient temperatures, and sink conditions. The results are plotted as an average of triplicate measurements. 


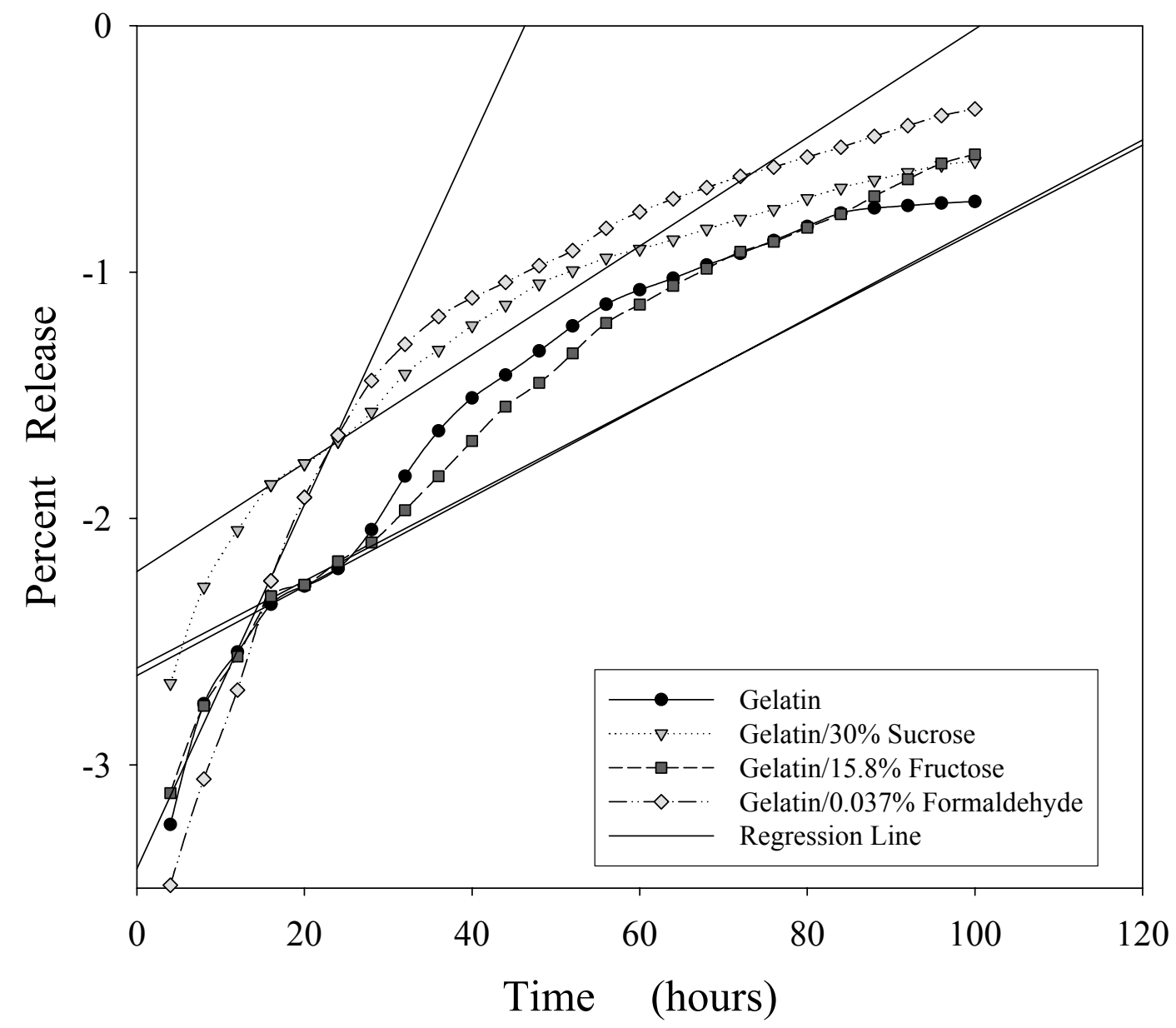

Figure 98. Linear regression for the first order equation over the second release phase (Hours 16-24) for matrices loaded with red dye.

Matrices were refrigerated for 24 hours followed by 24 hours of lyophilization and evaluated under excess moisture, ambient temperatures, and sink conditions. The results are plotted as an average of triplicate measurements. 


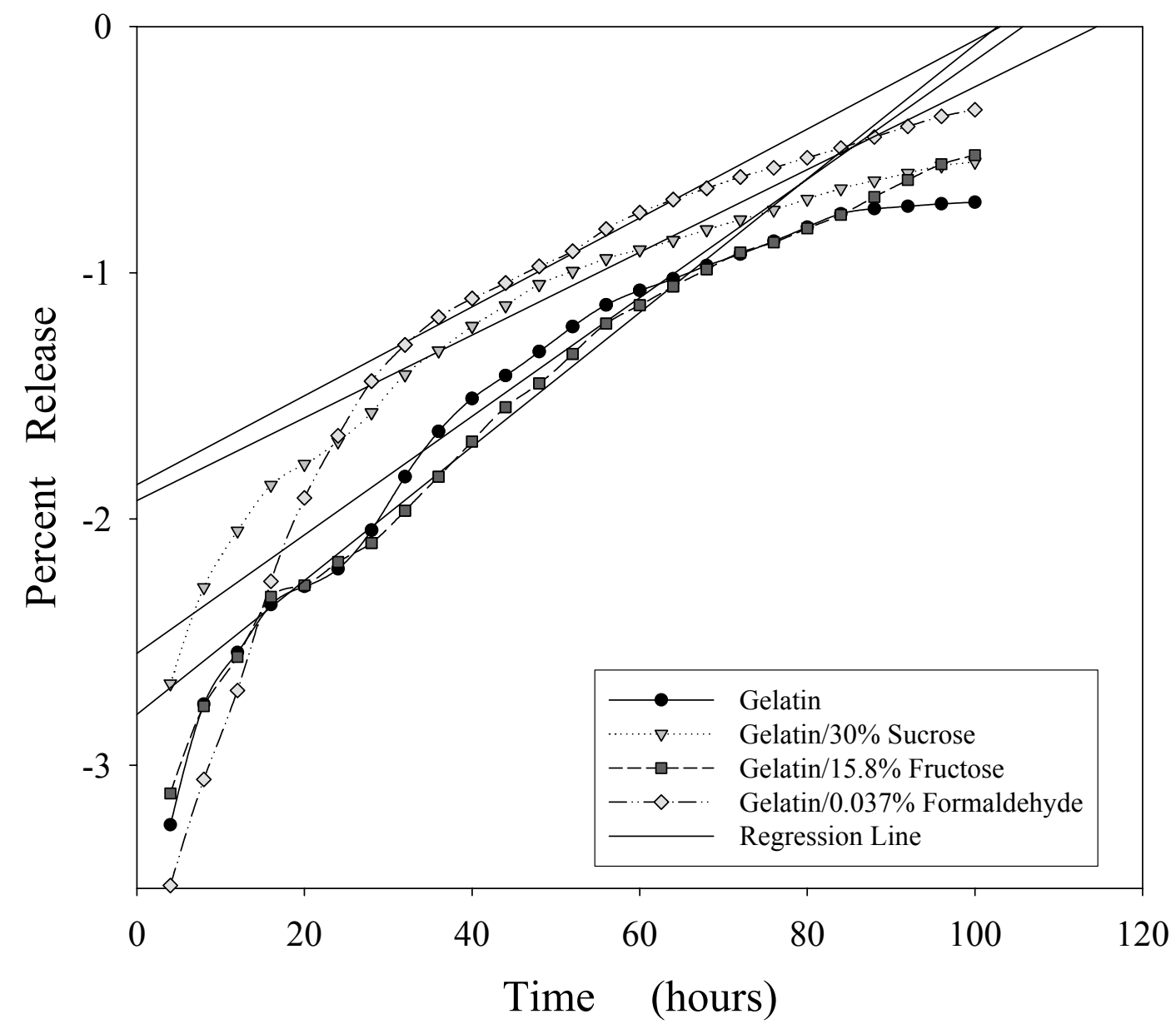

Figure 99. Linear regression for the first order equation over the third release phase (Hours 28-72) for matrices loaded with red dye.

Matrices were refrigerated for 24 hours followed by 24 hours of lyophilization and evaluated under excess moisture, ambient temperatures, and sink conditions. The results are plotted as an average of triplicate measurements. 


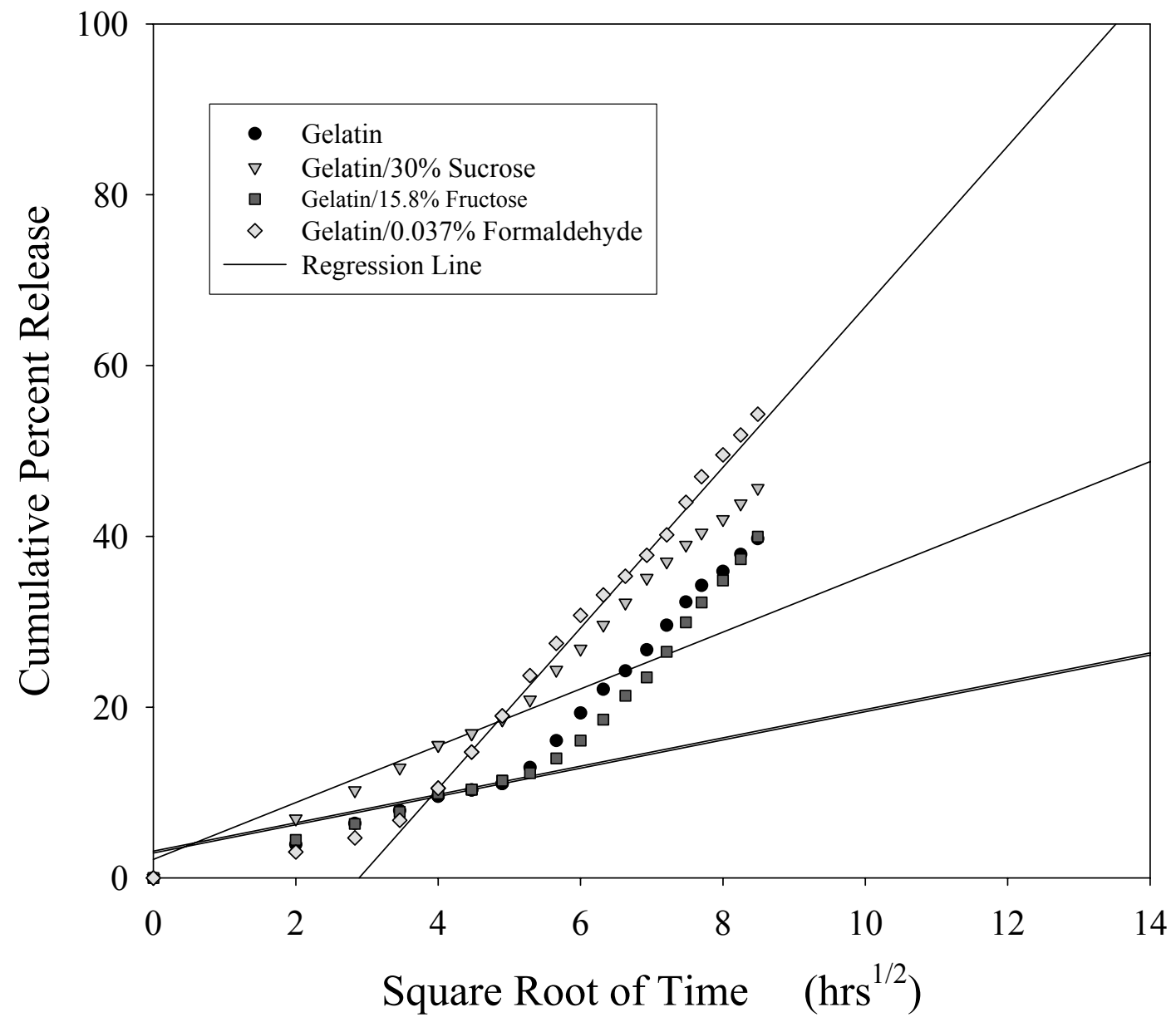

Figure 100. Linear regression for the Higuchi square root equation over the second release phase (Hours 16-24) for matrices loaded with red dye.

Matrices were refrigerated for 24 hours followed by 24 hours of lyophilization and evaluated under excess moisture, ambient temperatures, and sink conditions. The results are plotted as an average of triplicate measurements. 


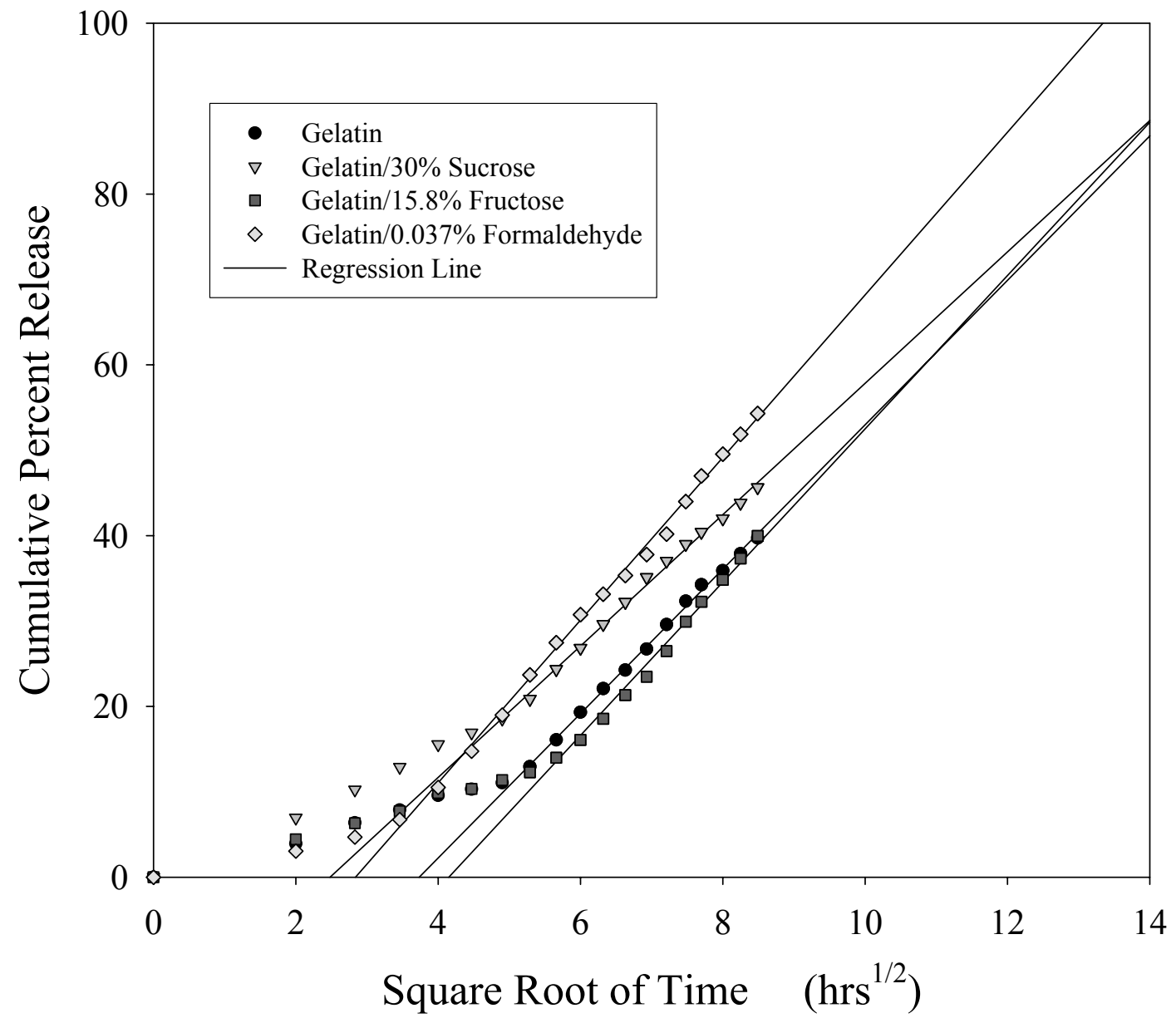

Figure 101. Linear regression for the Higuchi square root equation over the third release phase (Hours 28-72) for matrices loaded with red dye.

Matrices were refrigerated for 24 hours followed by 24 hours of lyophilization and evaluated under excess moisture, ambient temperatures, and sink conditions. The results are plotted as an average of triplicate measurements. 


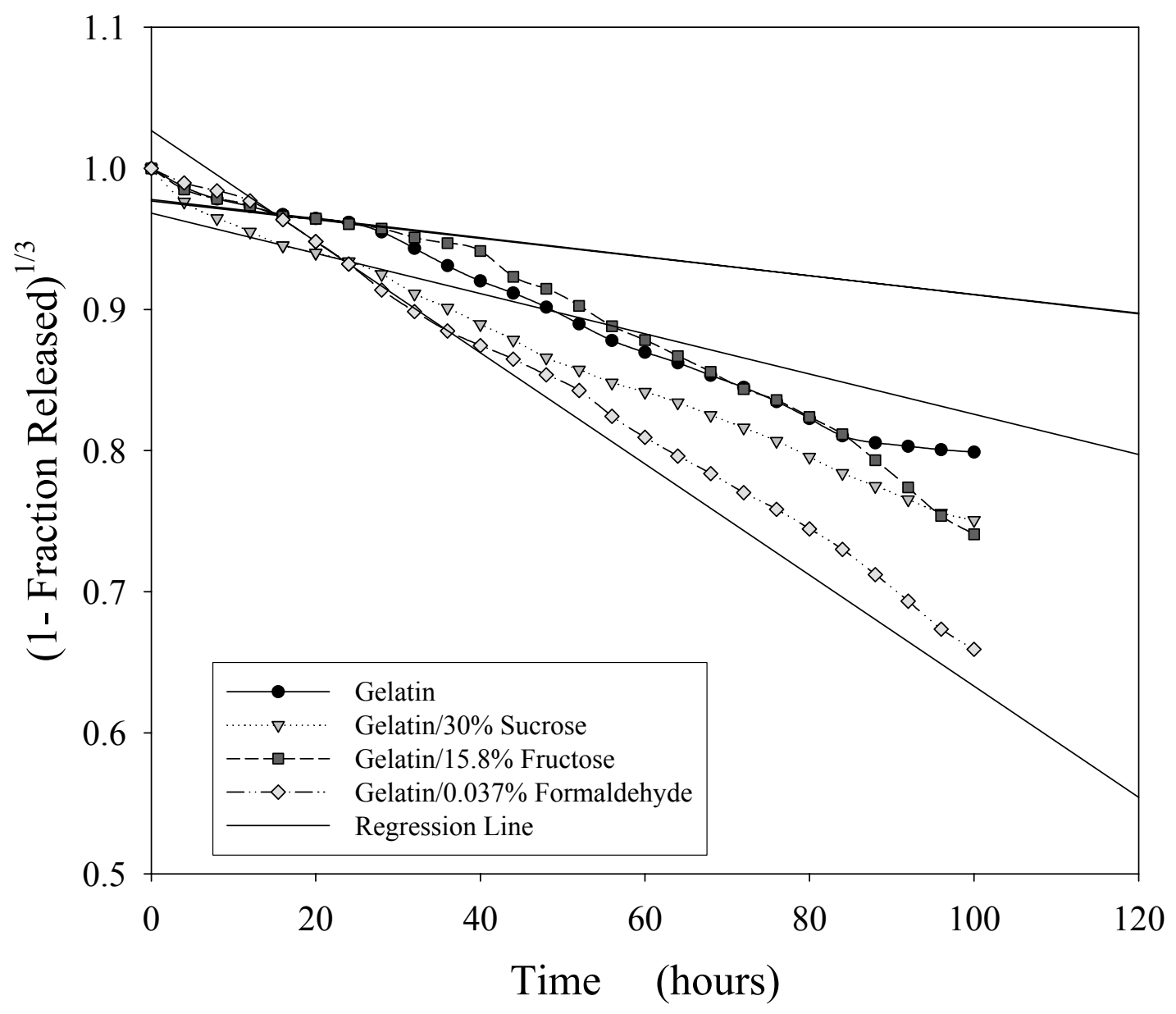

Figure 102. Linear regression for the Hixson-Crowell cube root equation over the second release phase (Hours 16-24) for matrices loaded with red dye.

Matrices were refrigerated for 24 hours followed by 24 hours of lyophilization and evaluated under excess moisture, ambient temperatures, and sink conditions. The results are plotted as an average of triplicate measurements. 


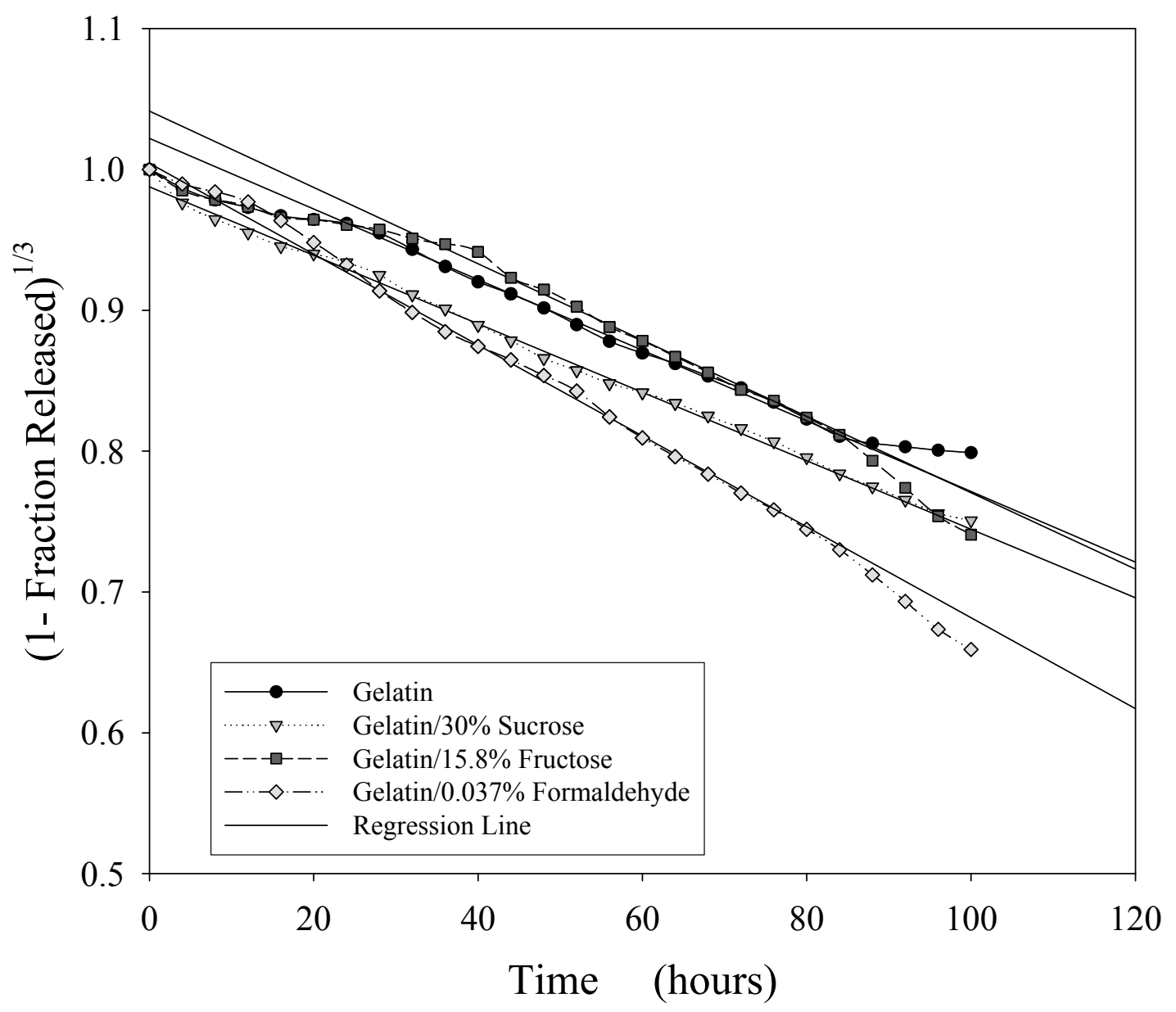

Figure 103. Linear regression for the Hixson-Crowell cube root equation over the third release phase (Hours 28-72) for matrices loaded with red dye.

Matrices were refrigerated for 24 hours followed by 24 hours of lyophilization and evaluated under excess moisture, ambient temperatures, and sink conditions. The results are plotted as an average of triplicate measurements. 
equation describe the dissolution of drug particles and do not consider release from a polymer matrix. Tables 16 and 17 show the regression data for the Higuchi model. Slopes for the phases were determined from the fit of the Higuchi model.

As with the more concentrated conditions, a third phase is seen in both the green and red loaded matrices. The gelatin, gelatin/sucrose, and gelatin/formaldehyde matrices loaded with green dye exhibit a slower diffusion phase. At this point in the release process, this could be due to water passing further into the matrix, through the matted arrangement of gelatin strands, and creating a gelatinous diffusional barrier that the dye must diffuse through. The gelatin/fructose matrices exhibit a slight increase in release, suggesting a loss of resistance to transport.

Comparison of the green and red release parameters show that the red loaded matrices exhibit a much slower release during the second phase. The third phase of the red dyes does not follow this same pattern of slowed diffusion as with the green dye. Instead, the rate of red dye increases in this terminal phase. This is different from that seen with release in a dye concentrated bulk solvent. This suggests that both the concentration gradient for the red dye is being maintained, and that the red dye gives some indication of matrix erosion in this phase. At this point in the release process, this could be due to the loss of gelatin matrix physical integrity (start of erosion) of the fructose formulation. 
Table 16. Comparison of the regression data for three diffusional phases of the green dye.

Data represents a mean of 3 determinations. HCHO represents formaldehyde.

\begin{tabular}{|c|c|c|c|}
\hline GELATIN & Slope & Y-intercept & Correlation \\
\hline Hours 12-32 & 0.0892 & -0.0125 & 0.9924 \\
Hours 36-52 & 0.0715 & 0.1176 & 0.9946 \\
\hline
\end{tabular}

\begin{tabular}{|c|c|c|c|}
\hline $\begin{array}{c}\text { GELATIN/ } \\
\text { SUCROSE }\end{array}$ & Slope & Y-intercept & Correlation \\
\hline Hours 12-32 & 0.0897 & 0.0920 & 0.9970 \\
Hours 36-52 & 0.0525 & 0.3214 & 0.9902 \\
\hline
\end{tabular}

\begin{tabular}{|c|c|c|c|}
\hline GELATIN/ & Slope & Y-intercept & Correlation \\
FRUCTOSE & 0.0830 & 0.0270 & 0.9904 \\
\hline Hours 12-32 & 0.1213 & -0.1769 & 0.9989 \\
Hours 36-52 & & \\
\hline
\end{tabular}

\begin{tabular}{|c|c|c|c|}
\hline $\begin{array}{c}\text { GELATIN/ } \\
\text { HCHO }\end{array}$ & Slope & Y-intercept & Correlation \\
\hline Hours 12-32 & 0.1108 & -0.1604 & 0.9990 \\
Hours 36-52 & 0.0699 & 0.0740 & 0.9928 \\
\hline
\end{tabular}


Table 17. Comparison of the regression data for three diffusional phases of the red dye.

Data represents a mean of 3 determinations. HCHO represents formaldehyde.

\begin{tabular}{|c|c|c|c|}
\hline GELATIN & Slope & Y-intercept & Correlation \\
\hline Hours 4-12 & 0.0273 & -0.0148 & 0.9960 \\
Hours 16-24 & 0.0165 & 0.0293 & 0.9986 \\
Hours 28-72 & 0.0846 & -0.3158 & 0.9981 \\
\hline
\end{tabular}

\begin{tabular}{|c|c|c|c|}
\hline $\begin{array}{c}\text { GELATIN/ } \\
\text { SUCROSE }\end{array}$ & Slope & Y-intercept & Correlation \\
\hline Hours 4-12 & 0.0408 & -0.0124 & 0.9998 \\
Hours 16-24 & 0.0333 & 0.0217 & 0.9943 \\
& 0.0769 & -0.1909 & 0.9956 \\
\hline
\end{tabular}

\begin{tabular}{|c|c|c|c|}
\hline $\begin{array}{c}\text { GELATIN/ } \\
\text { FRUCTOSE }\end{array}$ & Slope & Y-intercept & Correlation \\
\hline Hours 4-12 & 0.0225 & -0.0060 & 1.0000 \\
Hours 16-24 & 0.0166 & 0.0314 & 0.9982 \\
& & -0.3727 & 0.9938 \\
\hline Hours 28-72 & 0.0898 & & \\
\hline
\end{tabular}

\begin{tabular}{|c|c|c|c|}
\hline $\begin{array}{c}\text { GELATIN/ } \\
\text { HCHO }\end{array}$ & Slope & Y-intercept & Correlation \\
\hline Hours 4-12 & 0.0250 & -0.0210 & 0.9806 \\
Hours 16-24 & 0.0941 & -0.2717 & 0.9994 \\
Hours 28-72 & 0.0966 & -0.2808 & 0.9944 \\
\hline
\end{tabular}


Further evidence confirming the relative validity of the Higuchi model was obtained by utilizing the differential form of the equation $[9,10]$. The differential forms of the Higuchi model is $d\left(M_{t} / M_{\infty}\right)=f\left(1 /\left(M_{t} / M_{\infty}\right)\right)$. The Higuchi model was the only model in which the differential forms were evaluated in this section. This is due to the fact that the Higuchi model describes the release of drug from a polymer matrix while the other two models describe the dissolution of the drug particles. The linearity was obtained when $d\left(M_{t} / M_{\infty}\right)$ was plotted as a function of $1 / M_{t} / M_{\infty}$ further confirming that the process follows the Higuchi model, as seen in Figures 106-113. All three phases of both dyes show linearity when evaluating the differential forms. This also confirms that the Higuchi model best describes the overall release of dye from the gelatin-based matrices.

\section{Drug Studies}

Based on the sink and non-sink condition dye release data, the fluorescein data was evaluated using the Higuchi model (Figures 112 and 113). The fluorescein data does not fit the Higuchi model very well (Table 18). This supports the observation that the release of highly soluble, relatively neutral molecules may depend more on the swelling of the matrix rather than the diffusion of the drug out of the system. 


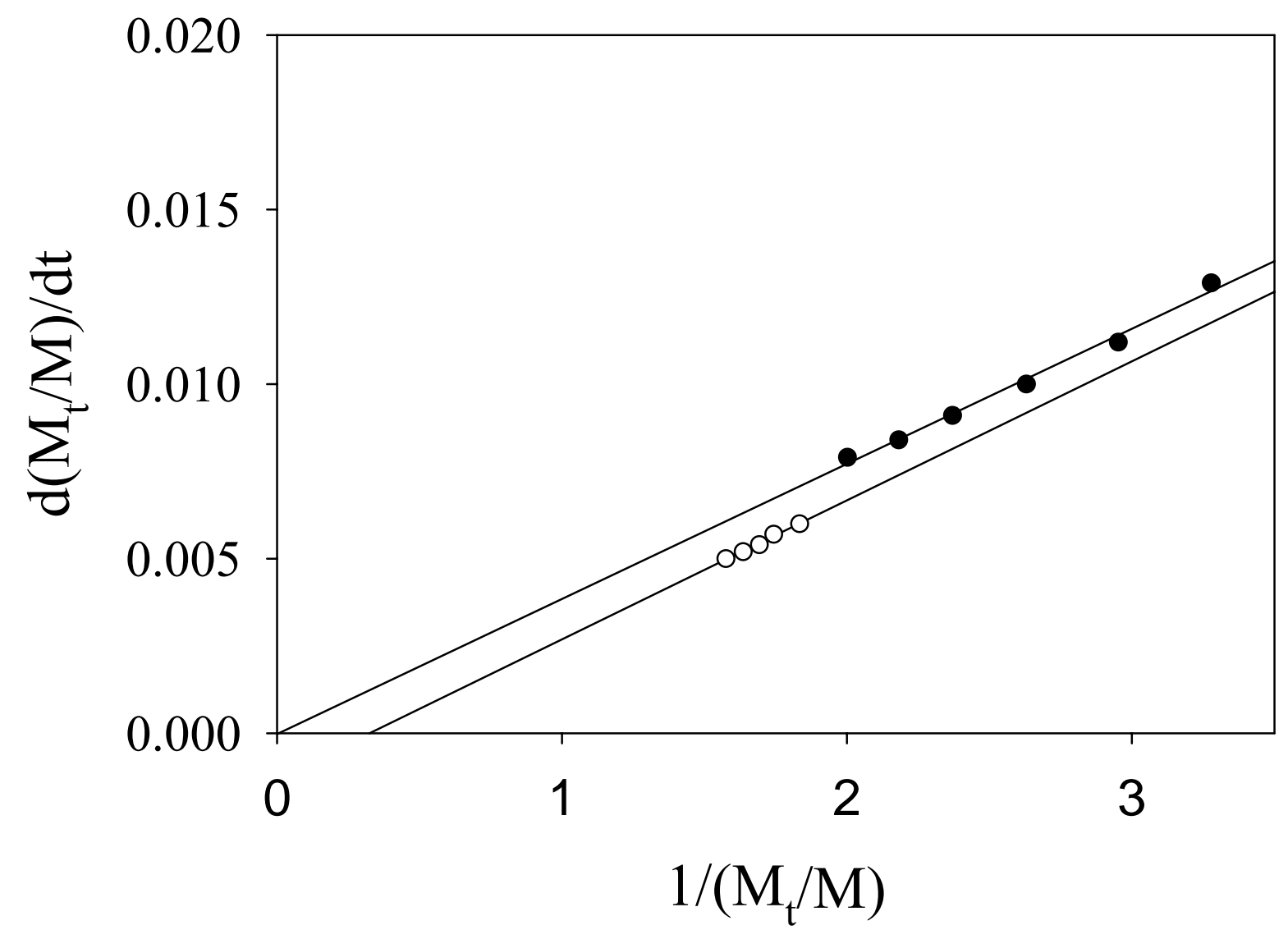

Figure 104. Plot of $d\left(M_{t} / M_{\infty}\right)$ versus $1 /\left(M_{t} / M_{\infty}\right)$ for the gelatin matrices loaded with green dye.

Matrices were refrigerated for 24 hours followed by 24 hours of lyophilization and evaluated under excess moisture, ambient temperatures, and sink conditions. The results are plotted as an average of triplicate measurements. 


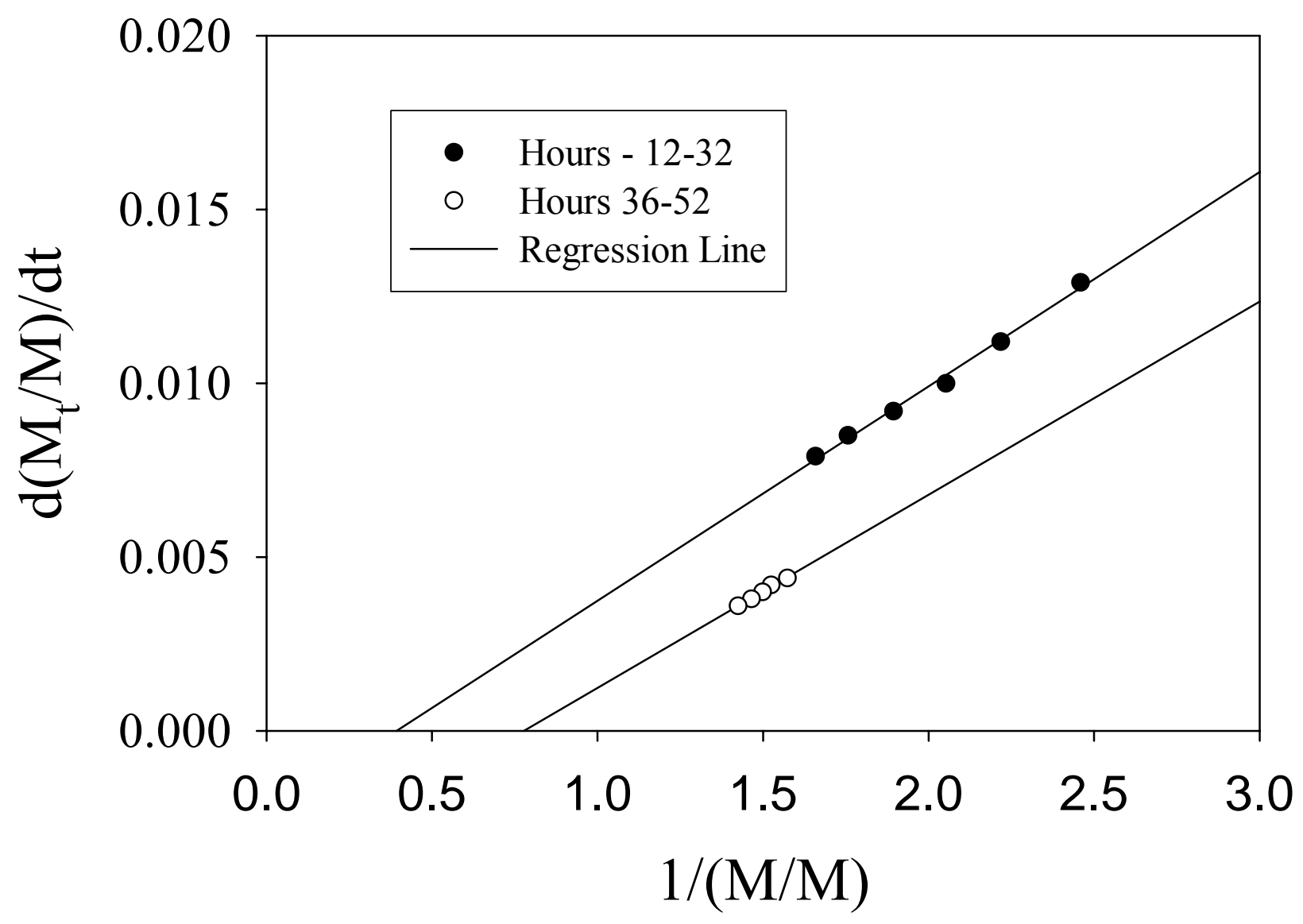

Figure 105. Plot of $d\left(M_{t} / M_{\infty}\right)$ versus $1 /\left(M_{t} / M_{\infty}\right)$ for the gelatin/30\% sucrose matrices loaded with green dye.

Matrices were refrigerated for 24 hours followed by 24 hours of lyophilization and evaluated under excess moisture, ambient temperatures, and sink conditions. The results are plotted as an average of triplicate measurements. 


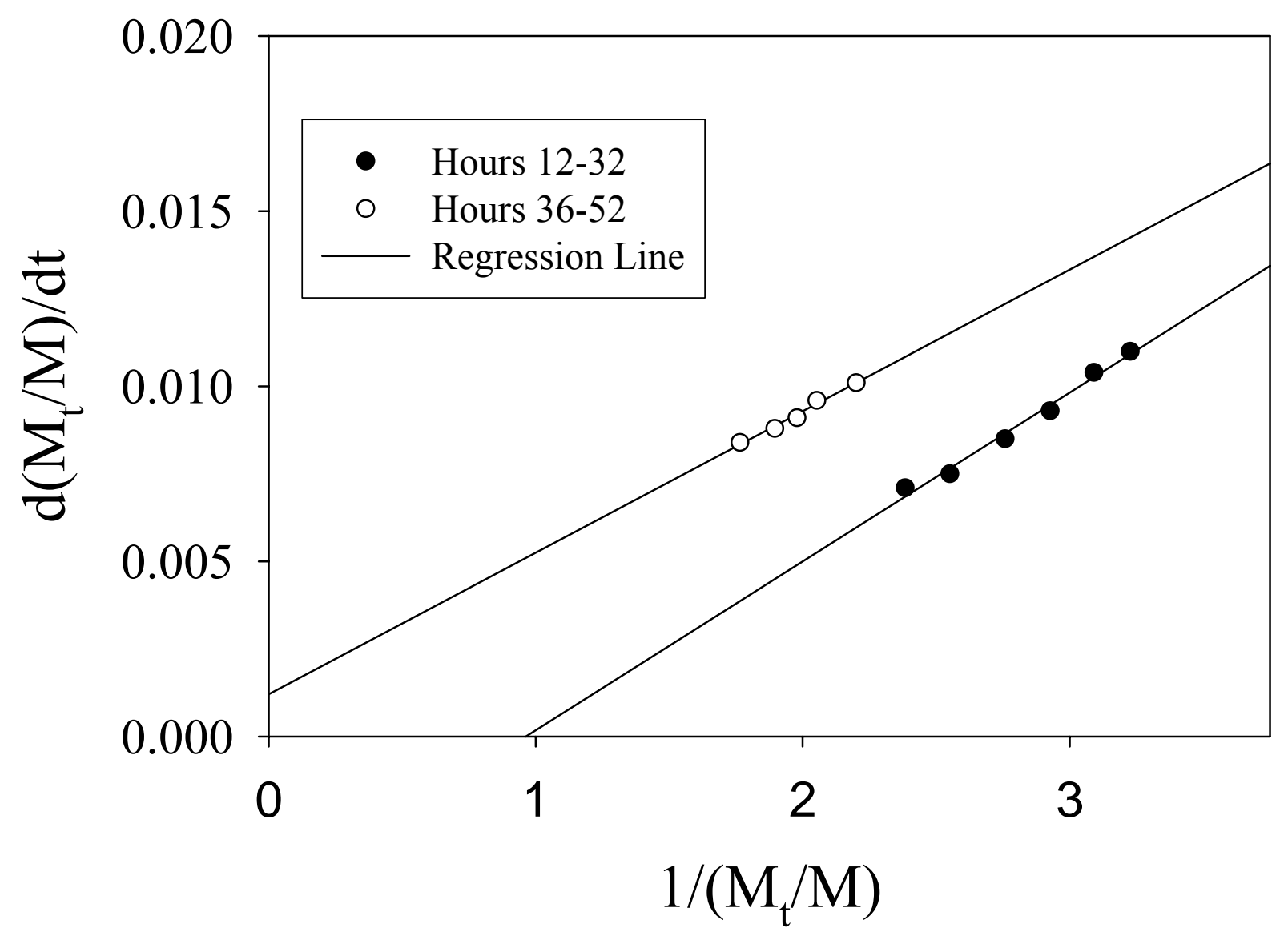

Figure 106. Plot of $d\left(M_{t} / M_{\infty}\right)$ versus $1 /\left(M_{t} / M_{\infty}\right)$ for the gelatin/15.8\% fructose matrices loaded with green dye.

Matrices were refrigerated for 24 hours followed by 24 hours of lyophilization and evaluated under excess moisture, ambient temperatures, and sink conditions. The results are plotted as an average of triplicate measurements. 


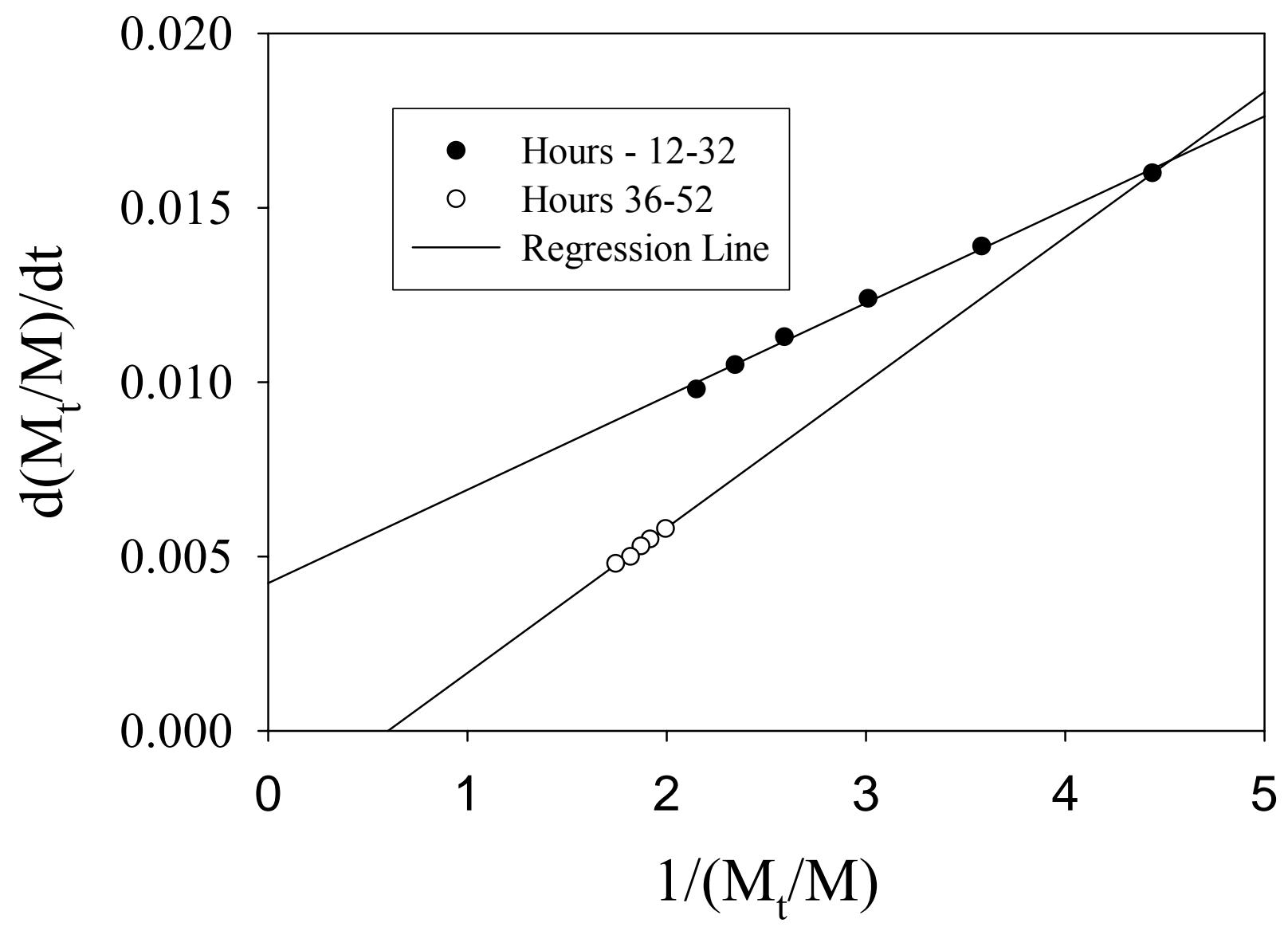

Figure 107. Plot of $d\left(M_{t} / M_{\infty}\right)$ versus $1 /\left(M_{t} / M_{\infty}\right)$ for the gelatin/0.037\% formaldehyde matrices loaded with green dye.

Matrices were refrigerated for 24 hours followed by 24 hours of lyophilization and evaluated under excess moisture, ambient temperatures, and sink conditions. The results are plotted as an average of triplicate measurements. 


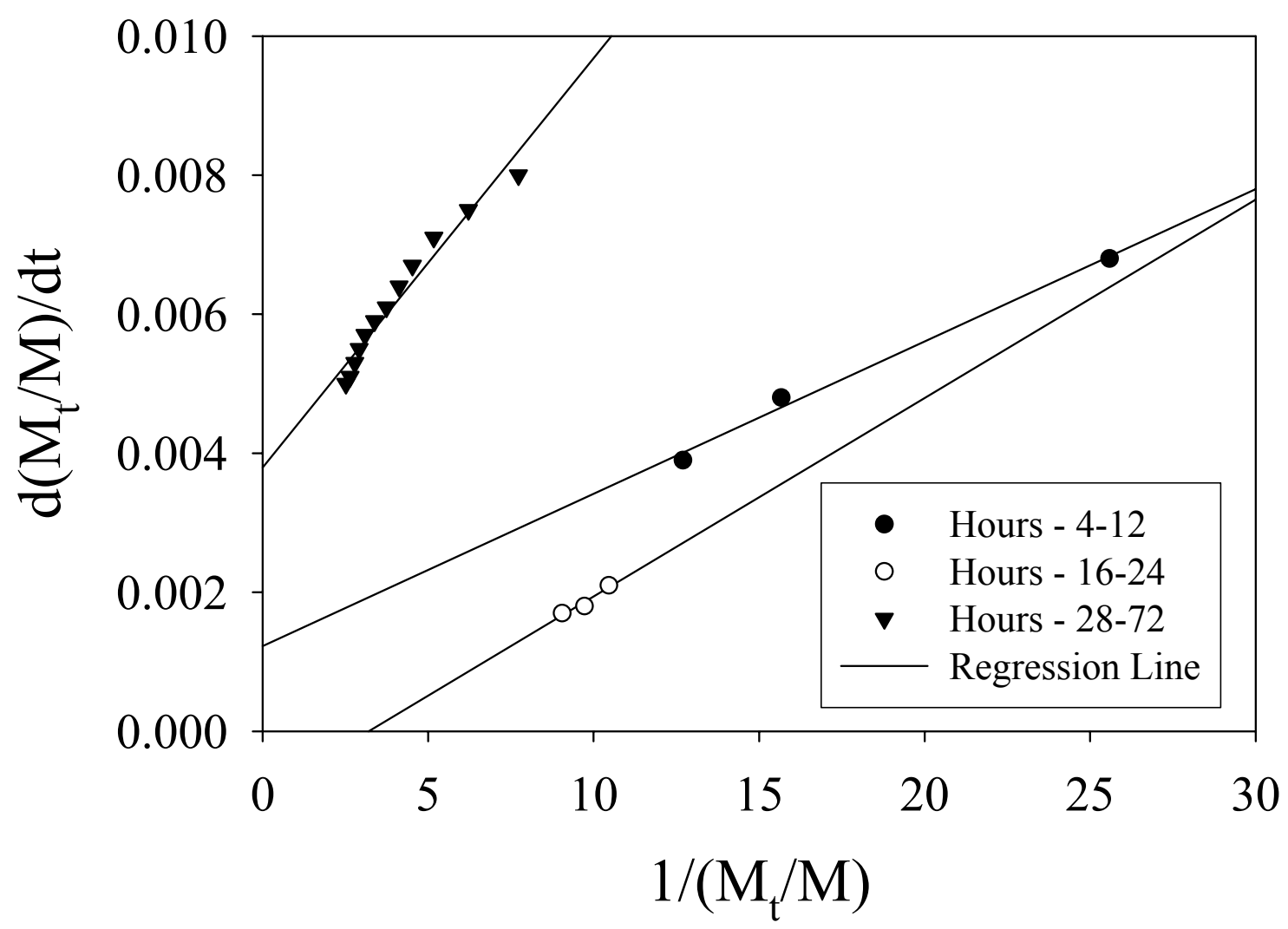

Figure 108. Plot of $d\left(M_{t} / M_{\infty}\right)$ versus $1 /\left(M_{t} / M_{\infty}\right)$ for the gelatin matrices loaded with red dye.

Matrices were refrigerated for 24 hours followed by 24 hours of lyophilization and evaluated under excess moisture, ambient temperatures, and sink conditions. The results are plotted as an average of triplicate measurements. 


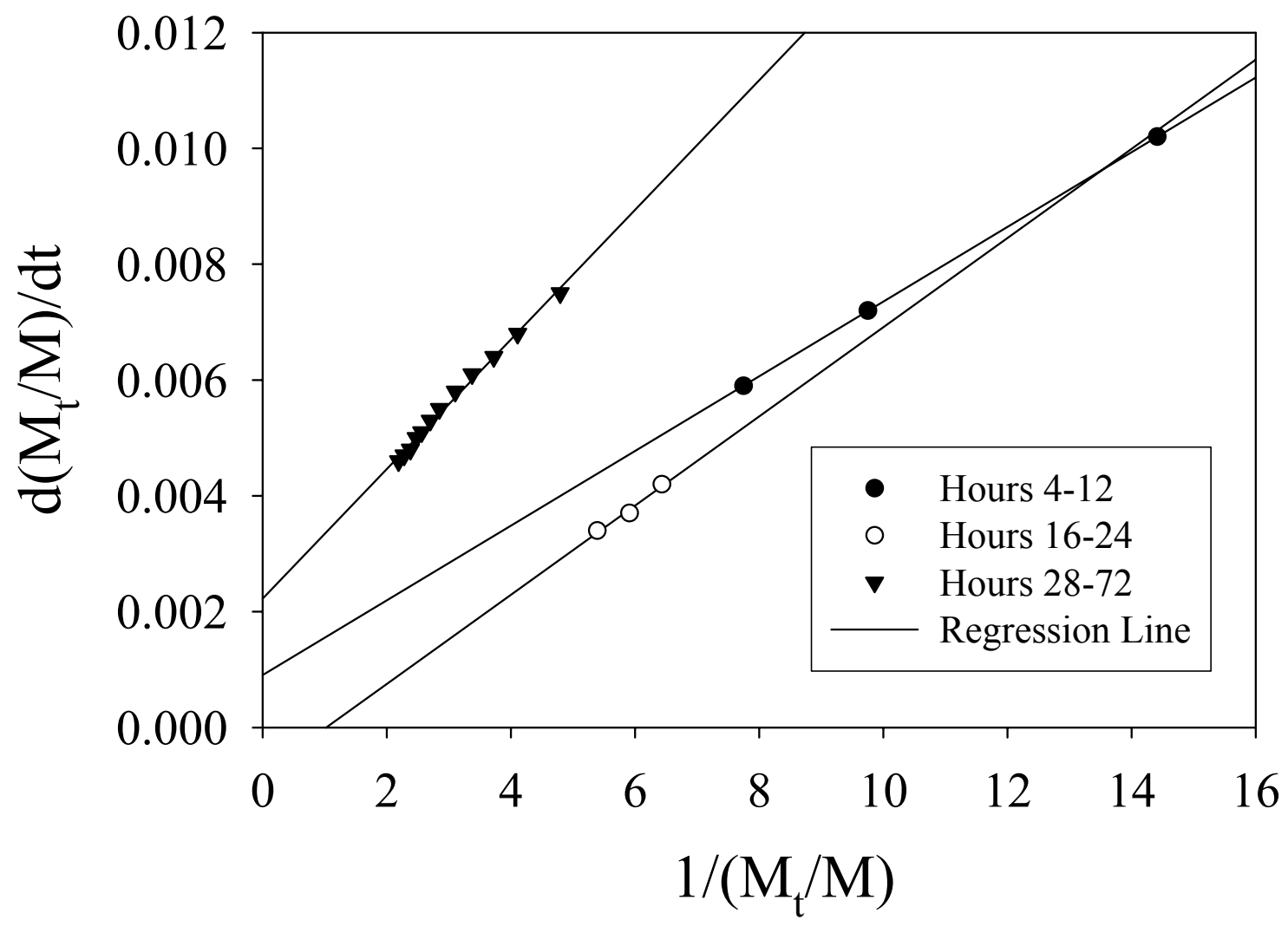

Figure 109. Plot of $d\left(M_{t} / M_{\infty}\right)$ versus $1 /\left(M_{t} / M_{\infty}\right)$ for the gelatin/30\% sucrose matrices loaded with red dye.

Matrices were refrigerated for 24 hours followed by 24 hours of lyophilization and evaluated under excess moisture, ambient temperatures, and sink conditions. The results are plotted as an average of triplicate measurements. 


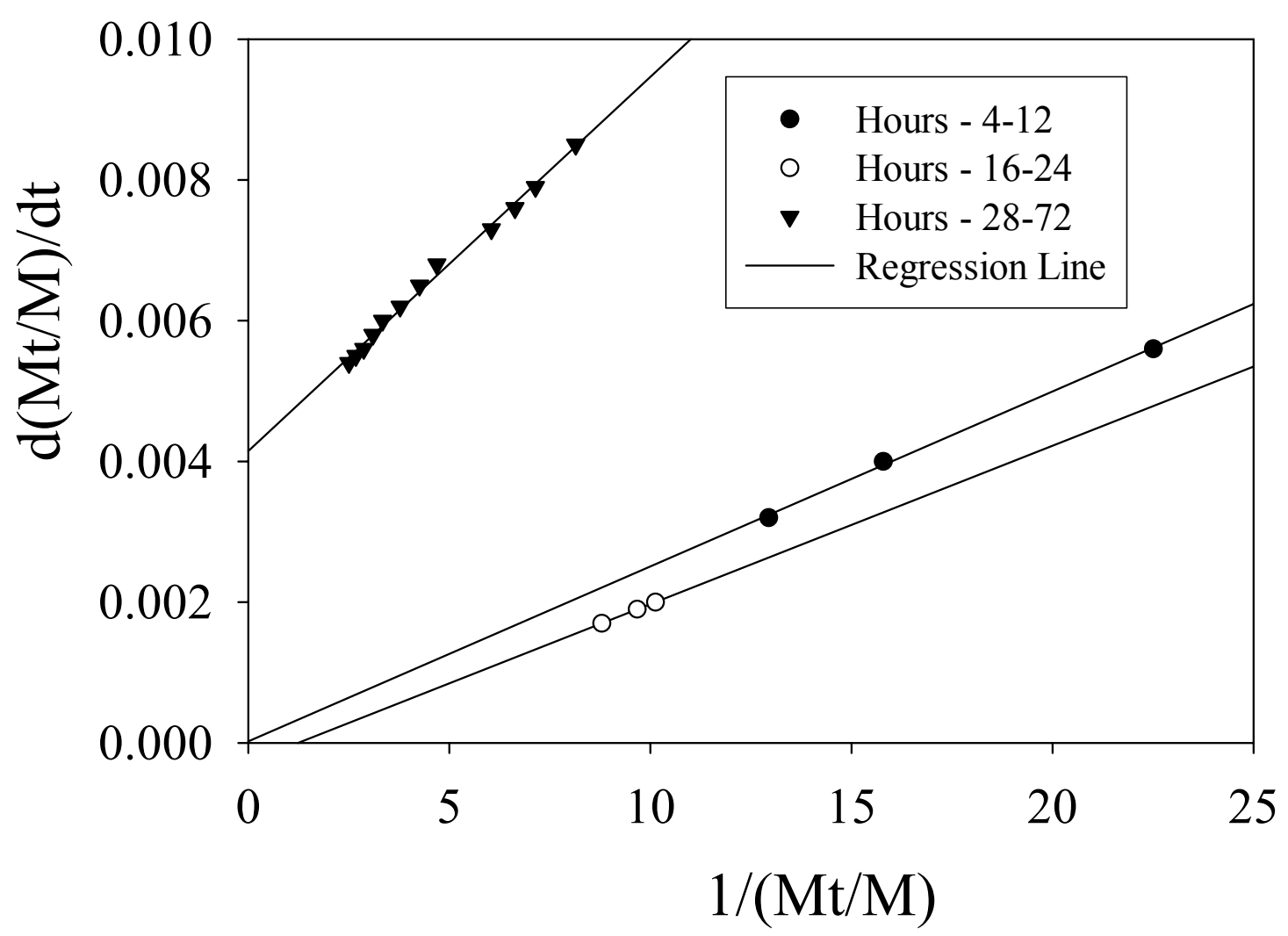

Figure 110. Plot of $d\left(M_{t} / M_{\infty}\right)$ versus $1 /\left(M_{t} / M_{\infty}\right)$ for the gelatin $/ 15.8 \%$ fructose matrices loaded with red dye.

Matrices were refrigerated for 24 hours followed by 24 hours of lyophilization and evaluated under excess moisture, ambient temperatures, and sink conditions. The results are plotted as an average of triplicate measurements. 


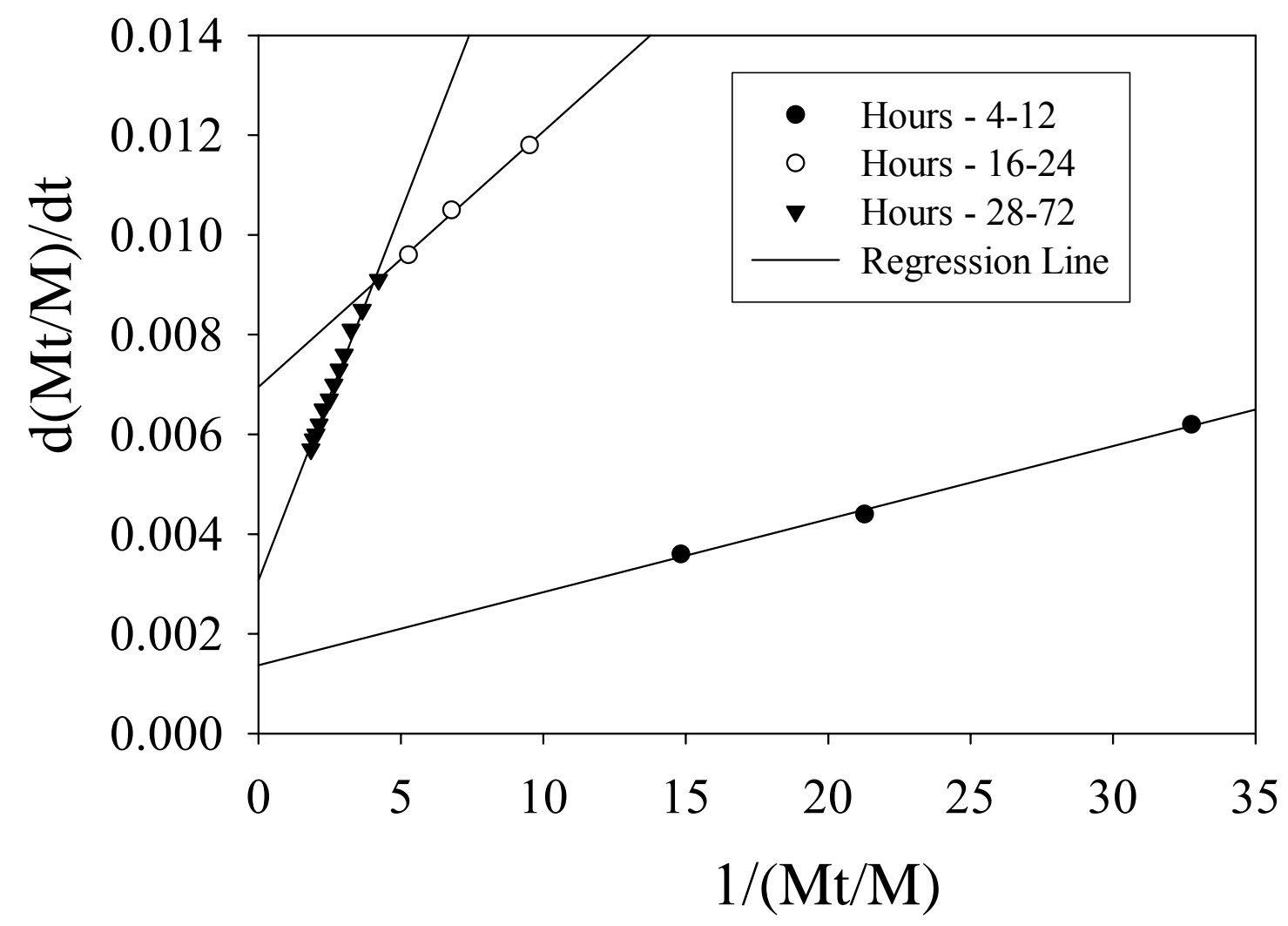

Figure 111. Plot of $d\left(M_{t} / M_{\infty}\right)$ versus $1 /\left(M_{t} / M_{\infty}\right)$ for the gelatin/0.037\% formaldehyde matrices loaded with red dye.

Matrices were refrigerated for 24 hours followed by 24 hours of lyophilization and evaluated under excess moisture, ambient temperatures, and sink conditions. The results are plotted as an average of triplicate measurements. 


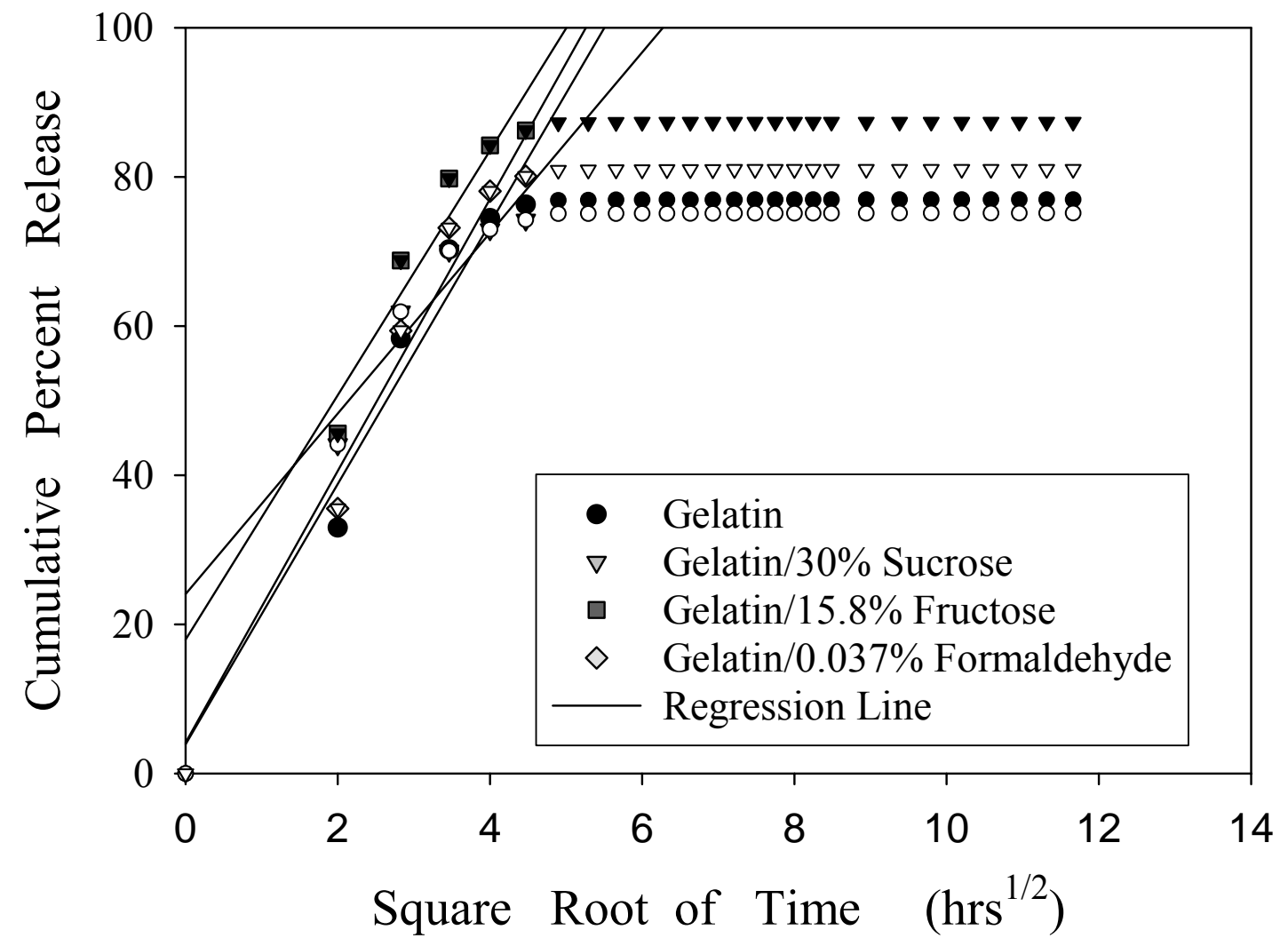

Figure 112. Linear regression for the Higuchi square root equation over the first phase (Hours 4-20) for matrices loaded with fluorescein.

Matrices were refrigerated for 24 hours followed by 24 hours of lyophilization and evaluated under excess moisture, ambient temperatures, and sink conditions. The results are plotted as an average of triplicate measurements. 


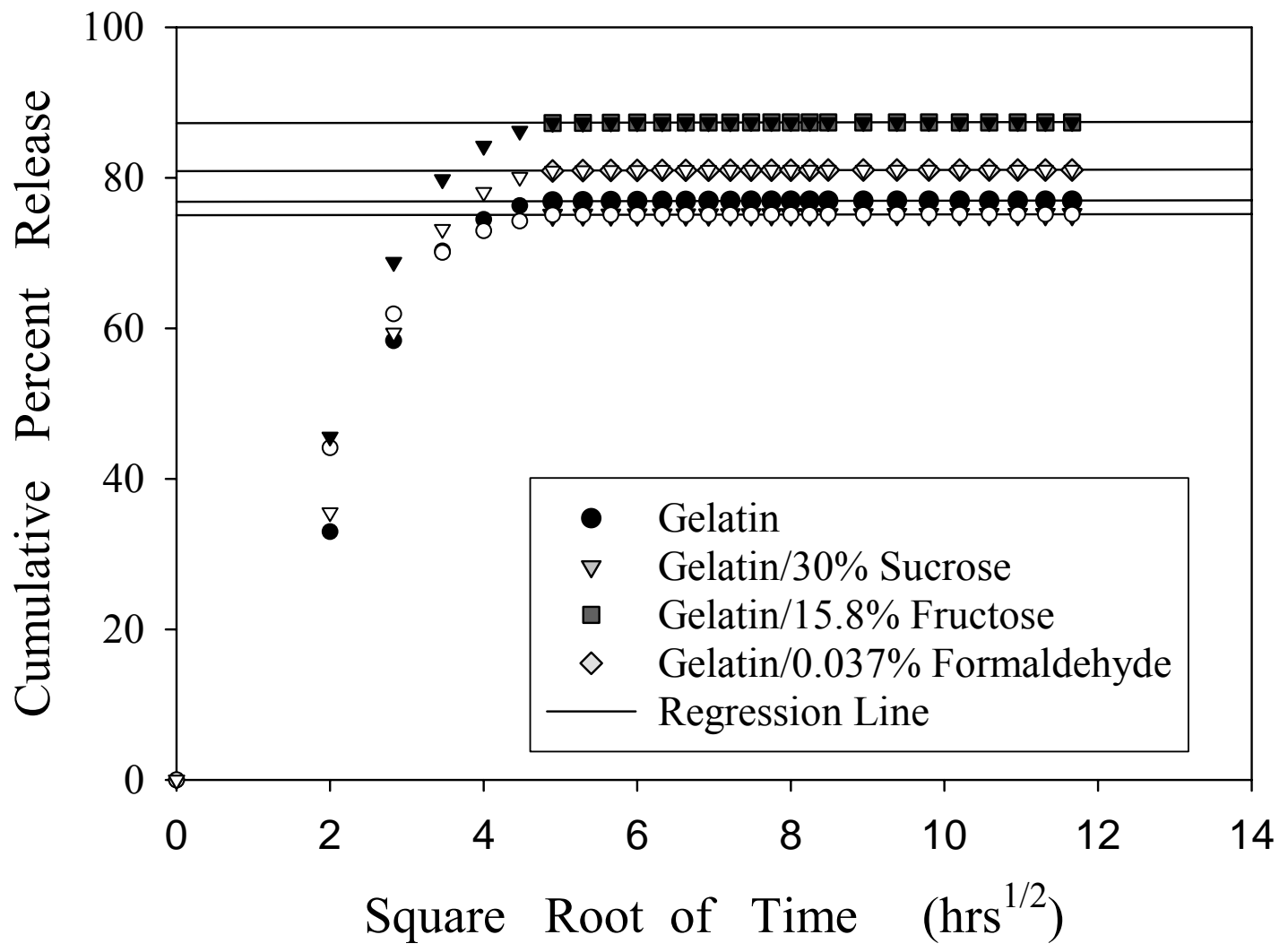

Figure 113. Linear regression for the Higuchi square root equation over the second phase (Hours 24-136) for matrices loaded with fluorescein.

Matrices were refrigerated for 24 hours followed by 24 hours of lyophilization and evaluated under excess moisture, ambient temperatures, and sink conditions. The results are plotted as an average of triplicate measurements. 
Table 18. Correlation coefficients and linear regression data for fluorescein loaded matrices.

Data represents a mean of 3 determinations. HCHO represents formaldehyde.

\begin{tabular}{|c|c|c|c|}
\hline GELATIN & Slope & Y-intercept & Correlation \\
\hline Hours 4-20 & 17.4600 & 3.9182 & 0.9008 \\
Hours 24-136 & 0.0131 & 76.8147 & 0.6835 \\
\hline
\end{tabular}

\begin{tabular}{|c|c|c|c|}
\hline $\begin{array}{c}\text { GELATIN/ } \\
\text { SUCROSE }\end{array}$ & Slope & Y-intercept & Correlation \\
\hline Hours 4-20 & 12.1085 & 24.0525 & 0.8983 \\
Hours 28-72 & 0.0106 & 75.0127 & 0.7649 \\
\hline
\end{tabular}

\begin{tabular}{|c|c|c|c|}
\hline $\begin{array}{c}\text { GELATIN/ } \\
\text { FRUCTOSE }\end{array}$ & Slope & Y-intercept & Correlation \\
\hline Hours 4-20 & 16.3890 & 17.9669 & 0.9121 \\
Hours 24-136 & 0.0124 & 87.2447 & 0.7448 \\
\hline
\end{tabular}

\begin{tabular}{|c|c|c|c|}
\hline $\begin{array}{c}\text { GELATIN/ } \\
\text { HCHO }\end{array}$ & Slope & Y-intercept & Correlation \\
\hline Hours 4-20 & 18.2245 & 4.1430 & 0.9224 \\
Hours 24-136 & 0.0158 & 80.8745 & 0.8252 \\
\hline
\end{tabular}




\section{Discussion}

In vitro release and modeling studies revealed that the release of dye from the matrices appears to be diffusion-controlled, with minimal affect of swelling on release kinetics.

Based on the three models presented in the beginning of this chapter, the Higuchi model best describes the release of dye from the gelatin-based matrices. The Higuchi model describes the release of drug from a polymer matrix while the other two models describe the dissolution of the drug particles. The first order and Hixson-Crowell models do not take into account the path length in which the drug must travel in order to be released from the matrix. The Higuchi model also takes into account that release from the matrix follows a two step process. This correlates with the visual data, presented in the previous chapter, which has shown an initial release followed by a second, slower release.

All three models are based on the assumption that perfect sink conditions are maintained throughout the drug release profile. Sink conditions were not maintained during the course of dye release in the first set of data presented. The lack of sink conditions produces a profile that will reach a plateau over time as a result of the loss of the concentration gradient. Under sink conditions, the difference between $c_{s}$ and $c_{t}$ is large compared to non-sink conditions where the difference $\mathrm{c}_{\mathrm{s}}$ and $\mathrm{c}_{\mathrm{t}}$ is small. This results in a decrease in the diffusion rate of drug out of the matrix. Sink conditions were difficult to maintain for the reason that the concentrations of the dyes were not known. The dyes utilized in this study were purchased as a pre-formulated dye solution from 
McCormick \& Co, Inc (Hunt Valley, MD). The lack of sink conditions in the first set of data may have led to both a diminishing concentration gradient that then increased resistance to the dye's outward diffusion. In the second set of studies, sink conditions were bettered maintained by changing the dissolution medium every 4 hours. The loss of the concentration gradient is not seen in this set of studies, as the extent and rate of dye release is greater under these conditions.

The models presented here have some limitations due to the complexity of the polymer system. These models predict solute transport as varying functions of concentration. The three models do not incorporate elements of either a swelling or erosion model that consider both solvent and matrix effects. Swelling and erosion effects can play a major role at the beginning and end of the release profile, respectively. Furthermore, a surface versus a bulk erosion of the matrix would lead to different effects on drug release. Visual observations of the films' erosion characteristics suggest that the gelatin/sucrose and the gelatin/fructose matrices may undergo surface erosion, while the gelatin and gelatin/formaldehyde matrices erode by a bulk mechanism. Thus, even though diffusion may be the driving force in these phases, diffusion may not be the rate limiting step. Another limitation is that the three models do not consider any interactions that may be taking place between the gelatin, sugar, and/or drug/dye. The rate of diffusion can be greatly affected if the dye/drug is strongly bound to the gelatin or sugar either by a physical or chemical bond. There is some evidence in this study's data to suggest that net charge differences between the molecules may be influencing dye/drug 
release. These types of interactions could potentially inhibit the time it takes to reach a steady-state by several hours.

Of the three models evaluated, the Higuchi model best predicts the release of dye from the four formulations, and provides the closest approximate fit. Both sink and nonsink conditions support that diffusion is the driving force for dye release from all formulations. The use of dilute and concentrated conditions offers some insight into the clinical significance of the sugar-based matrices. These conditions mimic the conditions of disease states in which this delivery system could be utilized, a site with constant fluid turnover (delivery on the surface of the eye) or a site where the drug may build up in the fluids (delivery within the periodontal pocket).

The use of the pre-formulated dyes have made it difficult to completely characterize the release characteristics of these gelatin-based matrices. Further studies will need to be done to better understand the release characteristics of the gelatin, gelatin/sugar, and gelatin/formaldehyde matrices. These studies should include evaluating the release characteristics of prototype drugs that are currently being used in the treatment of periodontal disease as well as ophthalmic disorders. These therapeutic agents should include neutral as well as charged compounds and low and high solubility compounds. Basic mechanistic studies should also be performed to assess the extent of which net charge differences (attraction or repulsion) between matrix/drug influence drug release. 


\section{References}

1. Tomlinson E. Microsphere delivery systems for drug targeting and controlled release. Int J Pharm Tech Prod Manufact 1983;4:49-53.

2. Chowdhury DK, Mitra AK. Kinetics of in vitro release of model nucleoside deoxyuridine from crosslinked insoluble collagen and collagen-gelatin microspheres. Int J Pharm 199;193:113-122.

3. Ritger PL, Peppas NA. A simple equation for description of solute release II. Fickian and anomalous release from swellable devices. J Control Rel 1987;5:37-42.

4. Harland RS, Gazzaniga A, Sangalli ME, Colombo P, Peppas NA. Drug/polymer matrix swelling and dissolution Pharm Res 1988;5:488-494.

5. Amiji MM. Mass transport. In: Amiji MM, Sandmann BJ, editors. Applied Physical Pharmacy. New York:McGraw-Hill, 2003. p. 285-310.

6. Abdou HM, Hanna S, Muhammad N. Dissolution. In: Gennero AR, editor. Remington: The science and practice of pharmacy. Baltimore, MD:Lippencott Williams \& Wilkins, 2000. p. 654-656.

7. Martin A, Swarbrick J, Cammarata A. In: Physical Pharmacy. Philadelphia:Lea \& Febiger, 1983. p. 399-444.

8. Higuchi T. Mechanism of sustained-action medication. J Pharm Sci 1963;52(12):1145-1149.

9. Samuelov Y, Donbrow M, Friedman M. Sustained release of drugs from ethylcellulose-polyethylene glycol films and kinetics of drug release. J Pharm Sci 1979;68(3):325-329.

10. Leucuta SE. Controlled release of nifedipine from gelatin microspheres and microcapsules: in vitro kinetics and pharmacokinetics in man. J Microencap 1990;7(2):209-217. 


\section{Chapter VIII}

\section{Summary and Conclusions}

The global objective of this research was to apply a material science-based approach to develop a localized delivery system utilizing gelatin as a drug carrier which bioerodes and provides prolonged drug release in a reproducible and predictable fashion. The best polymer candidates for sustained release delivery systems provide slow swelling and prolonged erosion.

The first specific goal of this work evaluated the potential of two sugars (sucrose and fructose) as cross-linking agents, replacing the more toxic aldehydes. Varying concentrations of the sugars in the formulation were assessed. The gelatin/sucrose and gelatin/fructose matrices demonstrated significant improvement in swelling/erosion behavior in comparison to non-cross-linked matrices. Gelatin/sugar matrix performance is sensitive to the cross-linking level and curing conditions, which promote water loss from the matrix. In an attempt to decrease the amount of water used in the gelatin dispersion, ethanol was used to substitute a percentage of water. The use of ethanol as a dispersing agent offers no advantage over the water-based formulations, as the matrices eroded more quickly. Thermal analysis, supported by matrix erosion studies, has revealed the affect of the sugars on the cross-linking and increased structure and stability of the matrices. These studies demonstrated that sucrose and fructose are viable alternatives to the aldehydes as cross-linking agents for gelatin/collagen-based matrices. 
Due to gelatin's sensitivity to curing conditions, several processing variables were assessed for their ability to form a strong and dry matrix. The refrigeration and vacuum drying process demonstrated performance improvements over curing under ambient conditions; however, these required long curing times (6-7 days and 4 days, respectively). These limitations led to the addition of a third processing approach, lyophilization. Overall, the lyophilization process improved the performance of all matrices, reducing the curing time to 2 days. A comparison of multiple refrigeration and lyophilization times revealed that the swelling and erosion profiles of matrices refrigerated for 24 hours followed by 24 hours of lyophilization best fits the ideal profile of an initial burst followed by a plateau, while these curing conditions minimized the matrix processing time.

The second goal of the research was to compare and evaluate the efficacy of the sugars as cross-linking agents to a more traditional cross-linking agent, formaldehyde. The gelatin/sucrose and gelatin/fructose matrices demonstrated significant improvement in swelling behavior, but had a shorter time to erosion. After the initial swelling phase, the gelatin/sucrose and gelatin/fructose matrices reach a plateau, in which the matrices reach a static condition where the matrix ceases to swell (reaches maximum water content), but has no or minimal erosion. The gelatin/formaldehyde and non-cross-linked matrices never reach the plateau before absorbing a sufficient amount of solvent to cause disintegration of the matrix. 
Based on the results of both formulation and process optimization studies reported in Chapters III-V, the optimized formulations were developed using the following conditions: $15 \%$ non-cross-linked gelatin, $15 \%$ gelatin $/ 30 \%$ sucrose, $15 \%$ gelatin $/ 15.8 \%$ fructose, $15 \%$ gelatin $/ 0.037 \%$ formaldehyde matrices (with water as the dispersion agent), cured for 24 hours under refrigerated conditions followed by 24 hours of lyophilization.

The third goal was to assess the drug release characteristics of the gelatin-based matrices and test models to identify those properties contributing to drug release. Visual release studies have demonstrated that the release of dye occurs over the entire swelling and erosion profile. At the point in which the matrices break down, the matrices still retained a slight tint of dye. The red loaded matrices appeared to retain more dye than the green loaded matrices. Visual studies demonstrated that two different solutes transport out of the matrices at different rates. At ambient temperatures and excess moisture, all four formulations remained intact until the matrix reached an aqueous saturation point, at which time the matrices became maximally swollen, resulting in the disintegration of the matrix. The gelatin, gelatin/sucrose, and gelatin/fructose matrices remained intact for 2024 days while the gelatin/formaldehyde matrices lasted 30-35 days. At physiological temperatures and limited moisture, the gelatin, gelatin/sucrose, and gelatin/fructose matrices remained intact for 12-24 hours while the gelatin/formaldehyde lasted for $>3$ days. 
The rate and extent of swelling does not appear to play a major role in the release of dye from the gelatin-based matrices. For fluorescein release, the rate and extent of swelling does seem to play a role in the release. For fluorescein, the shape of the release profile is similar in shape to the swelling profile. This indicates that swelling may play a role in the release of fluorescein (or other highly soluble drugs) from the gelatin-based matrices.

The release of the dye and fluorescein suggests that the solute release is a function, to some extent, of solute physicochemical properties. These include drug solubility, the salt form of the drug, net charge, and the surface area of the solid drug particle. The release profile of fluorescein, a highly soluble sodium salt, shows a rapid burst of fluorescein followed by a slow gradual release. On the other hand, the green and red dyes are less soluble and demonstrated a much slower release over time. The overall net charge of fluorescein is lower than the dyes, which may contribute to the quick release of fluorescein. The number of interactions between the dye and gelatin may be greater than with fluorescein leading to a slower release of the dye.

In vitro release studies demonstrated a biphasic pattern of release for both sink and concentrated conditions. The initial rate is related to the initial diffusion phase of the matrix, when first exposed to the water medium releasing, free dye on the matrix surface. For dye within the matrix pores or associated with the gelatin, a diffusion process becomes the main driving force, resulting in the second phase. A third phase appears later and is most likely due to a less favorable concentration gradient, that ultimately, 
results in a much slower release rate, in most cases. In vitro release and modeling studies revealed that the release of dye from the matrices appears to be diffusion-controlled. The evaluation of three diffusional models; first order, Higuchi square root of time, and Hixson-Crowell; have revealed that dye release follows the Higuchi square root of time model. The utilization of the differential form of the model further confirms that dye release follows the Higuchi square root of time model. Of the three models evaluated, the Higuchi model best predicts the release of dye from the four formulations, and provides the closest approximate fit. Evaluation of the fluorescein data indicates that release may be influenced by the swelling of the matrices. The use of swelling models to evaluate this data should be considered in future studies.

The advantage of gelatin-based matrices is the ability to develop a delivery system that can utilize geometry differences as a function of delivery and delivery site needs. Of the two sugars, the gelatin/fructose matrices demonstrated the most potential due to desired performance materials characteristics (low percent swelling and prolonged erosion profile) and matrix stability (large peak area and high endothermic melting temperature). The release of dye from the gelatin/fructose matrices show a prolonged release throughout the swelling/erosion profile and retains a small quantity of dye at the time of erosion. While the sugars could not replace formaldehyde in formulations needed for extended sustained release delivery ( $>2$ days), the sugars offer a better alternative in formulations requiring an extended release of drug over 12-48 hours and offer the advantage of minimized risk of toxic damage to soft tissue at the delivery site. 


\section{APPENDIX A}

\section{Identification of Cross-links}

To date, there have been several proposed mechanisms of gelatin cross-linking but few direct methods have been identified which can measure the extent of crosslinking. Digenis, et al [1] proposed several mechanisms in which all of the reactions involve $\varepsilon$-amino groups of lysine. Bubnis, et al [2] modified a previous assay [3-4] utilizing 2, 4, 6-trinitrobenzenesulfonic acid (TBNS) to determine the concentration of $\varepsilon$ amino groups. This assay is a step toward defining the extent of cross-linking but has its limitations. The assay provides a reliable measure of the $\varepsilon$-amino groups; however, it is limited to a small range of sample weights and is dependent on the number of $\varepsilon$-amino groups per molecule or protein. This assay does not take into account any other chemical interactions that may take place within the matrix, such as arginine-arginine interactions.

Traditional techniques, such as x-ray diffraction, neutron scattering, and solutionstate NMR, can measure intermolecular distances between atoms. However, these techniques have limitations. Scattering techniques are of limited use for amorphous systems such as glassy polymers and for large macromolecular systems lacking high quality crystals [5]. Solution-state NMR requires a liquid sample along with low molecular weight systems due to the solubility issues and spectral crowding. Due to the physical nature and complex system of the gelatin-based matrix, these techniques do not provide further insight for the extent of cross-linking. Solid-state NMR can provide 
structural information without the limits imposed by molecular weight and can be applied to crystalline, microcrystalline, and amorphous systems [5].

Solid-state NMR can be employed to identify any changes in state of the sugar cross-linked gelatin matrices. Digenis, et al [1] proposed that the aldehyde group of the sugar can react with a free $\varepsilon$-amino group on the side chains of the gelatin molecule producing a ketose sugar. The ketose sugar can then further react with another free $\varepsilon$ amino group and form the cross-linked product. Solid-state NMR can be employed to identify any changes in state of the sugar cross-linked gelatin matrices. The expected changes in the NMR spectra when comparing gelatin alone with sugar cross-linked gelatin include the appearance of a peak that represents the hydroxyl groups found on the sugars, a decrease in the aldehyde region due to the loss of aldehyde moiety on the sugars, and an increase in the amide peak as a result of the new amide bonds formed between the gelatin strands and the sugars. The comparison of gelatin powder to the gelatin matrix shows an increase in the number of amide bonds (data not shown).

To obtain NMR spectra, samples were sent to Dr. Terry Gullion in the Chemistry Department of West Virginia University.

Appendix 1a shows the NMR spectra of the gelatin matrix. The first set of peaks, between 0 and $2750 \mathrm{~Hz}$ represent the nitrogen region. The second peak, between 6,000 and 7,000 $\mathrm{Hz}$ represents the carboxyl region of the gelatin strands. Appendix $1 \mathrm{~b}$ shows the NMR spectra of the gelatin/15.8\% fructose matrix. Both the nitrogen and carboxyl 
region are present with addition of a 3,600 to $3,800 \mathrm{~Hz}$ peak. This peak represents the hydroxyl groups that are found on the sugar. A contrast of the two spectra (Appendix 1c) shows that there is an increase in the size of the nitrogen peak. This indicates that the number of nitrogen bonds has increased after the fructose and gelatin are allowed to cross-link over time. This confirms that the sucrose and fructose may interact with the $\varepsilon$ lysyl groups on the gelatin strand as reported by Digenis, et al [1]. In Appendix 2, the gelatin/formaldehyde matrix shows a similar NMR spectra to the non-cross-linked gelatin (Appendix 1a). This may be due to the extremely low concentration of aldehyde $(0.037 \%)$ present in the matrix. Due to the difficulty in packing the gelatin/sucrose sample, no NMR spectra of the gelatin/sucrose matrix is available.

In conclusion, analysis of the NMR spectra indicate that new bonds are formed and the gelatin strands are connected through inter-strand and intra-strand bridges. Further studies need to be done in order to identify the specific amino acids involved in the cross-linking process. 
a
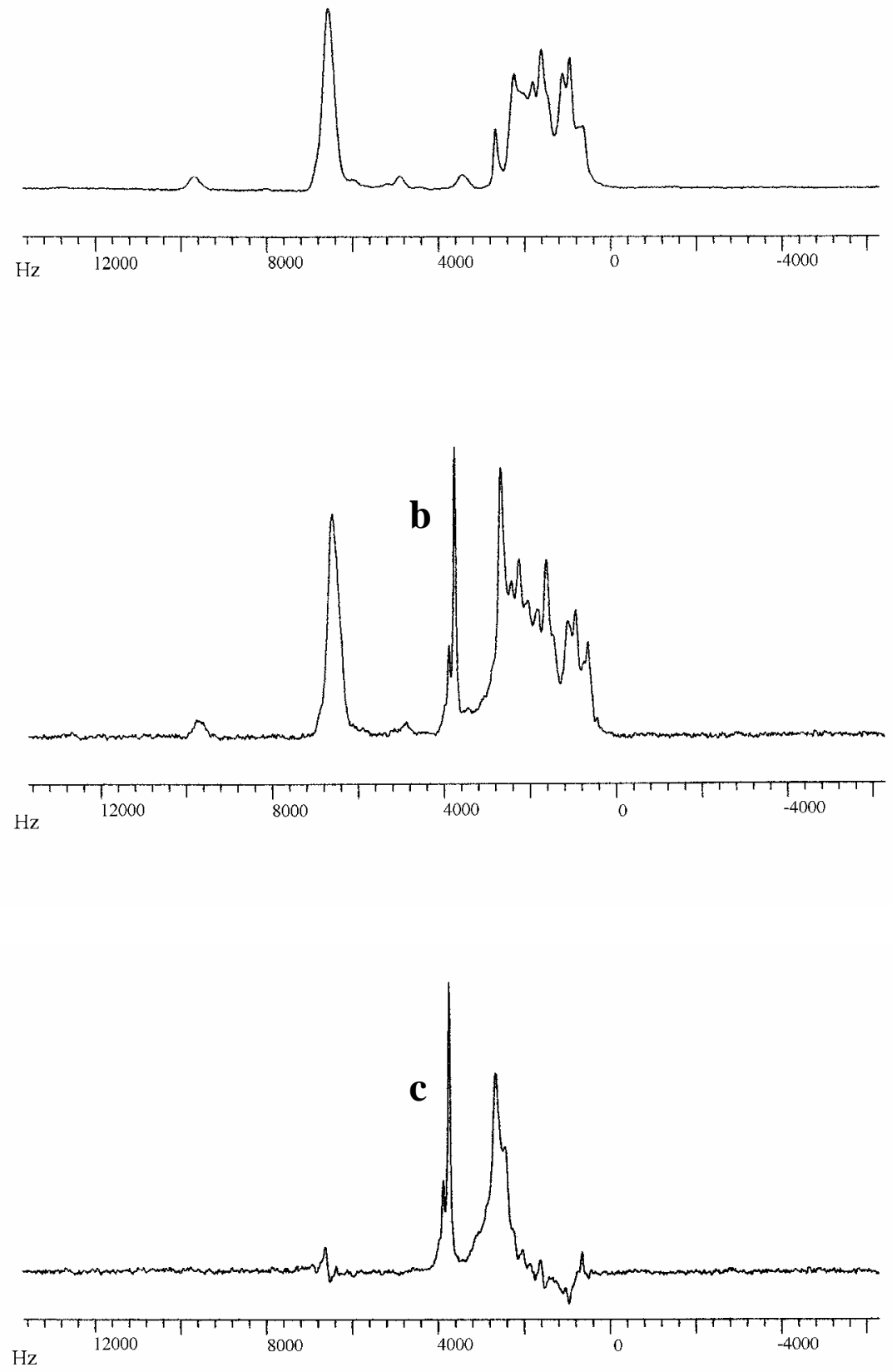

Appendix 1 NMR spectra of non-cross-linked gelatin (a), gelatin/15.8\% fructose (b), and difference of the gelatin and gelatin/15.8\% fructose (c). 


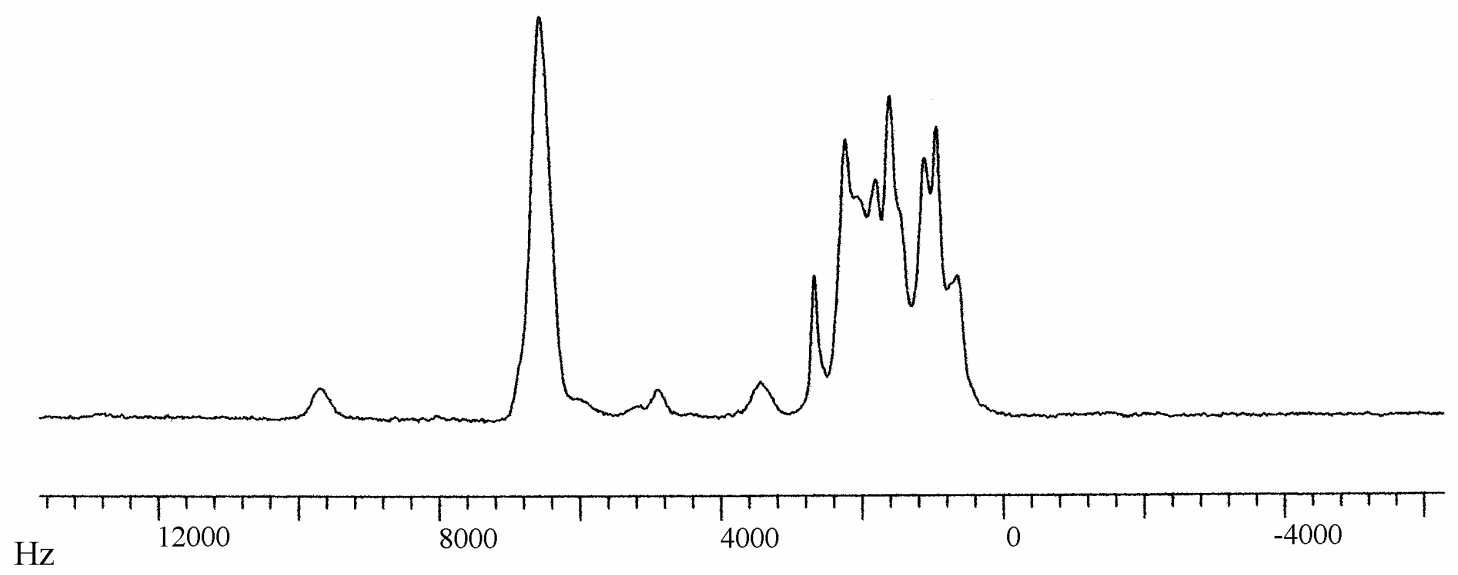

Appendix 2. NMR spectra of gelatin/0.037\% formaldehyde matrices. 


\section{References}

1. Digenis GA, Gold TB, Shah VP. Cross-linking of gelatin capsules and its relevance to their in vitro-in vivo peroformance. J Pharm Sci 1994;83(7):915-921.

2. Bubnis WA, Ofner III CM. The determination of $\varepsilon$-amino groups in soluble and poorly soluble proteinaceous materials by a spectrophotometric method using trinitrobenzenesulfonic acid. Anal Biochem 1992;207:129-133.

3. Gilbert DL, Okano T, Miyata T, Kim SW. Macromolecular diffusion through collagen membranes. Int J Pharm 1988;47:79-88.

4. Gilbert DL, Kim SW. Macromolecular release from collagen monolithic devices. $J$ Biomed Mater Res 1990;24:1221-1239.

5. Gullion T. Introduction to Rotational-Echo Double Resonance NMR. Concepts of Magnetic Resonance1998;10(5):277-289. 


\section{APPENDIX B}

\section{Standard Curves for Dye Markers}

Method: UV-Vis Spectrophotometer

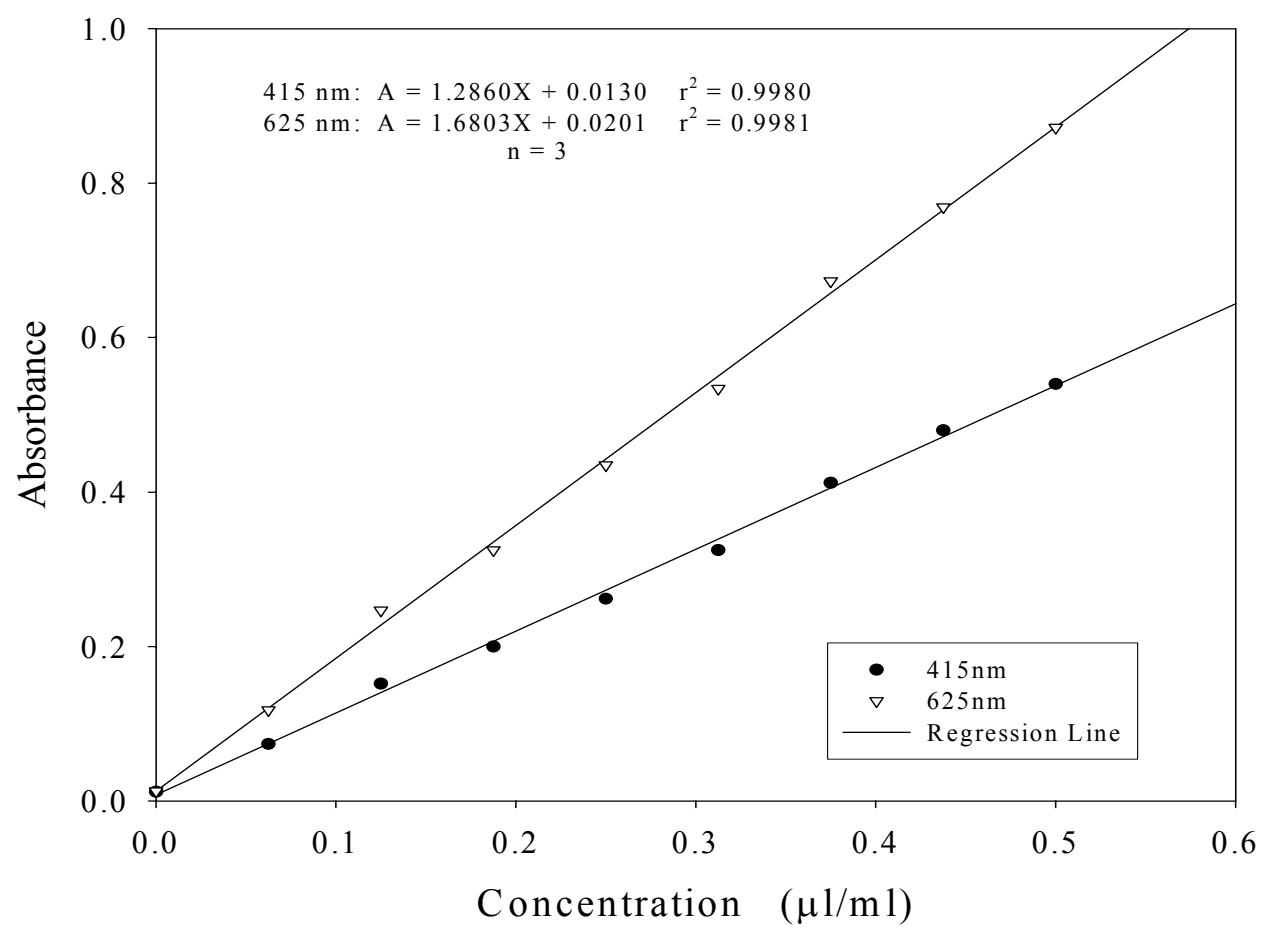

Appendix 3. Standard curve for green dye.

Wavelength $415 \mathrm{~nm}$ represents Tartrazine. Wavelength $625 \mathrm{~nm}$ represents Brilliant Blue. 


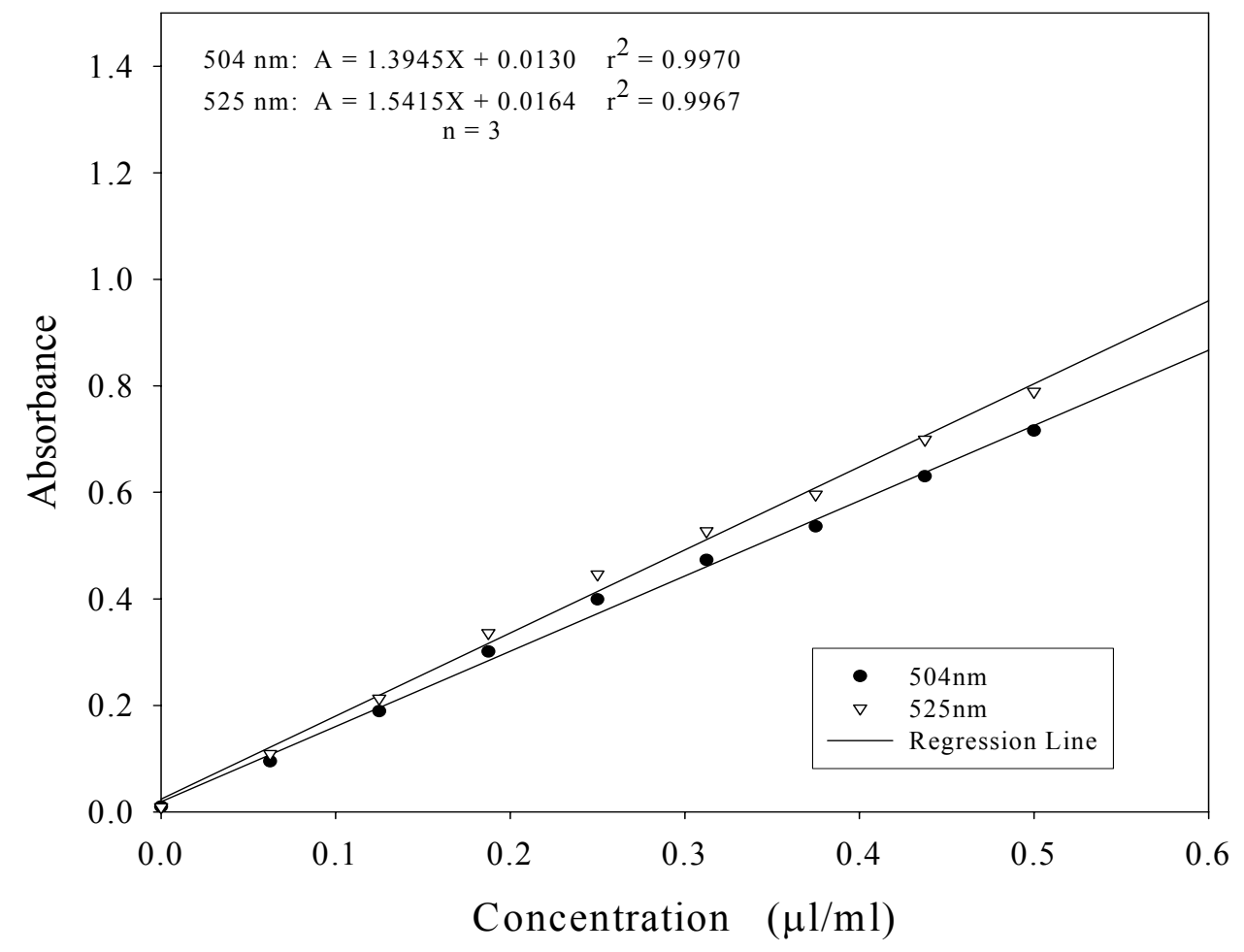

Appendix 4. Standard curve for red dye.

Wavelength $504 \mathrm{~nm}$ represents Allura Red. Wavelength $525 \mathrm{~nm}$ represents Erythrosine. 


\section{APPENDIX C}

Standard Curves for Fluorescein

Method: HPLC

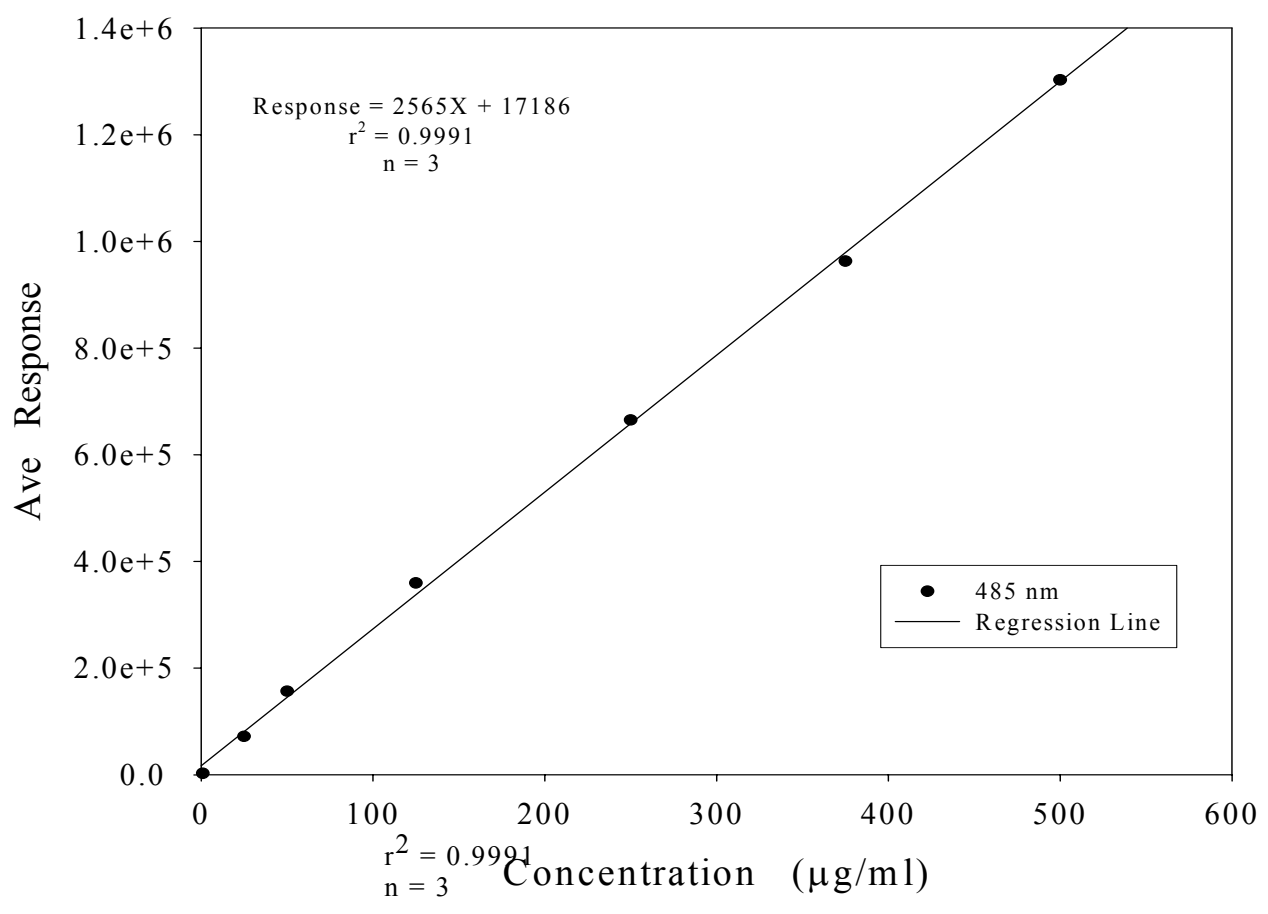

Appendix 5. Standard curve for high concentrations of fluorescein. 


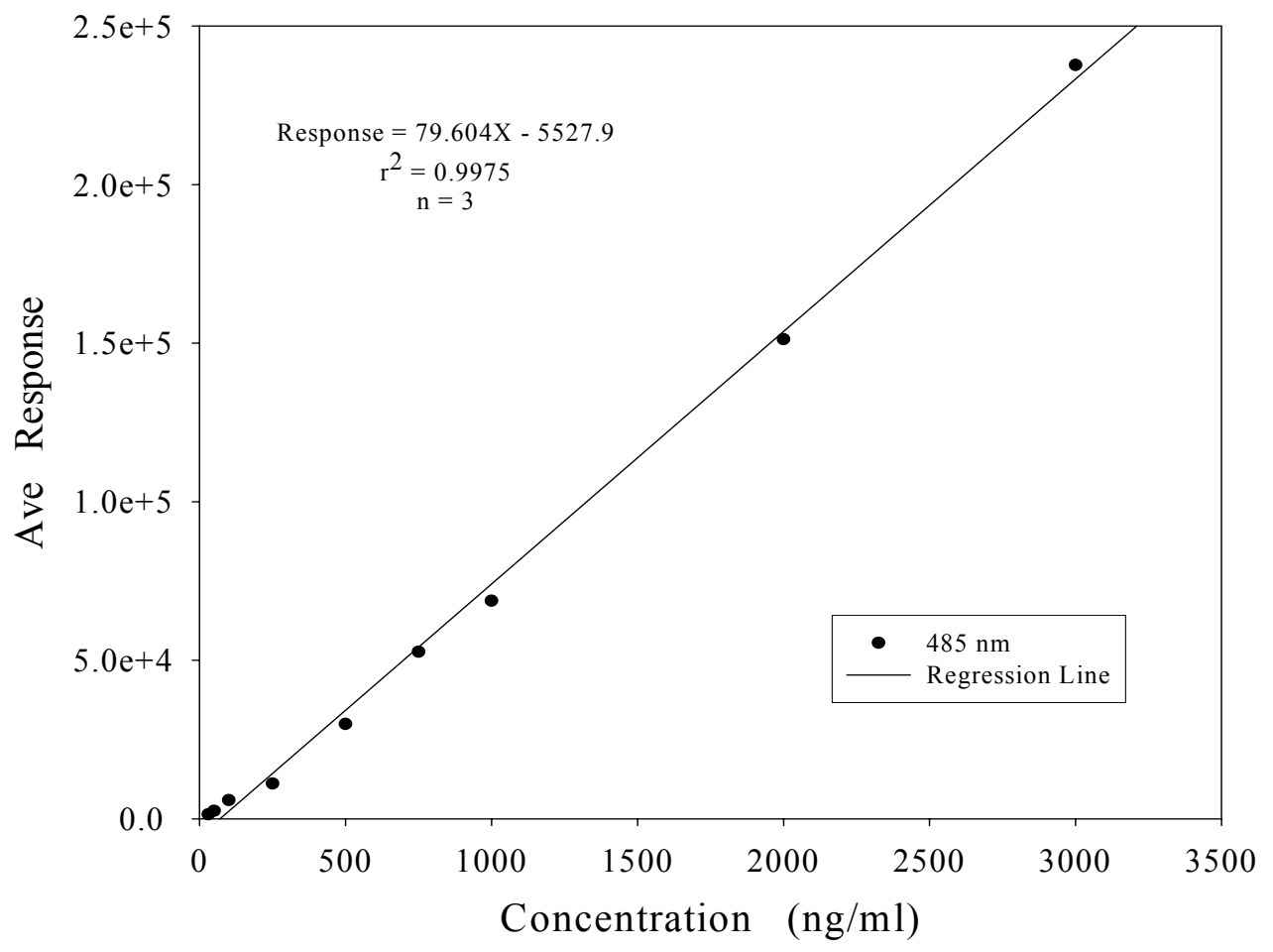

Appendix 6. Standard curve for low concentration of fluorescein. 

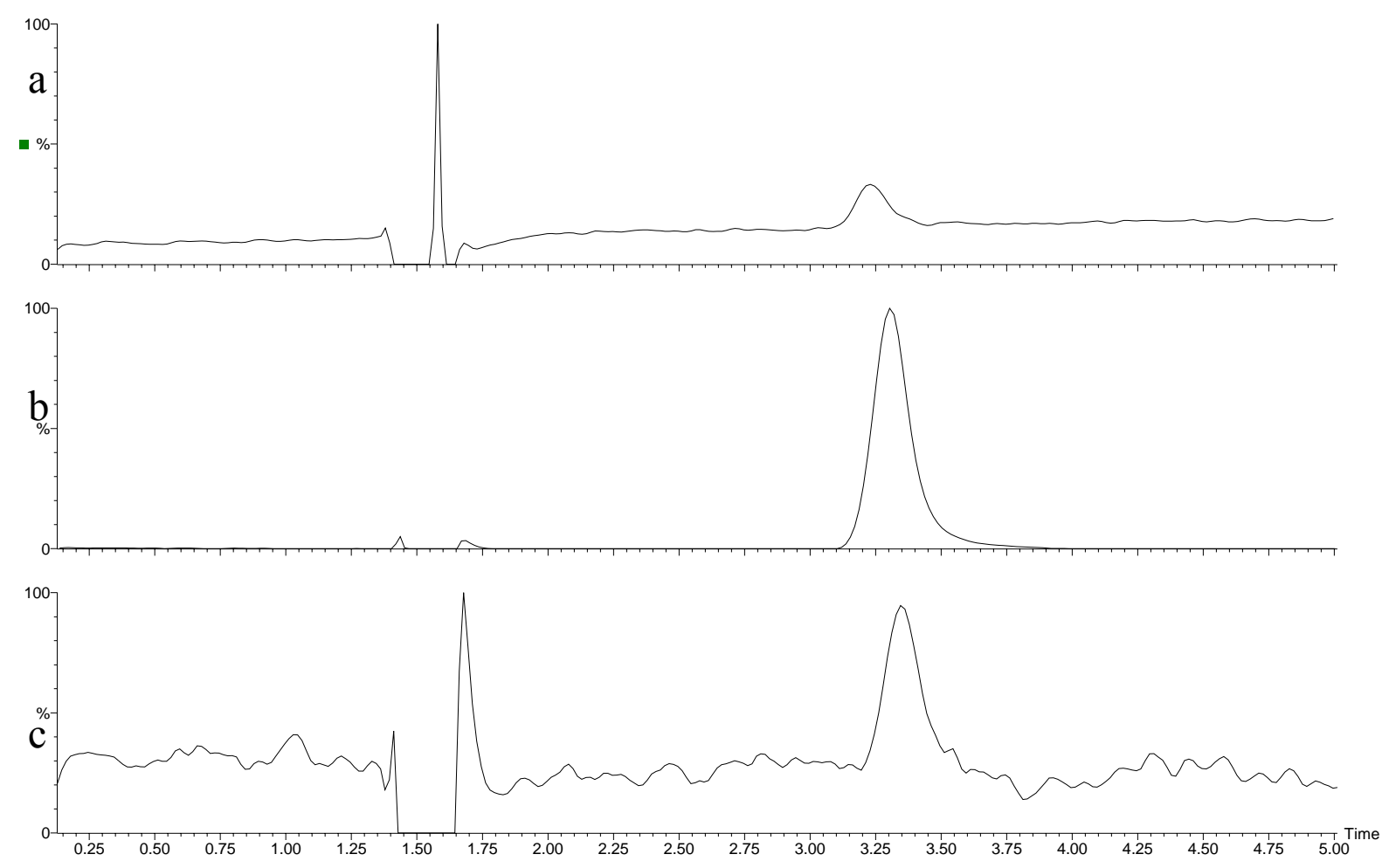

Appendix 7. Sample chromatograms for a $30 \mathrm{ng}$ standard (a), a $750 \mathrm{ng}$ standard (b), and an unknown sample (c) of fluorescein. 


\section{Brenda J. Schuler}

\section{EDUCATION}

West Virginia University, Morgantown, WV. Ph. D. Candidate in Basic

Pharmaceutical Sciences, expected graduation date December 2003.

Emphasis on formulation development and materials analysis.

Philadelphia College of Pharmacy and Science, Philadelphia, PA. B.S. in Pharmacy with a minor in Mathematics and Physics

\section{ACADEMIC HONORS}

Research Award, WVU School of Pharmacy- May 2002 - awarded based on poster presentation.

Teaching Award - WVU School of Pharmacy - May 2001 - awarded based on teaching portfolio.

\section{ADDITIONAL EDUCATION}

Pharmaceutical Technology Journal Discussion Group - Comprised of scientists from WVU Basic Pharmaceutical Sciences and Chemical Engineering Departments as well as Mylan Research and Development laboratories

Drexel University, Philadelphia, PA. Completed 9 credit hours of Physical Chemistry, 3 credit hours of Instrumental Analysis, and 9 credit hours towards an MBA.

Young Pharmacist Conference - May 1996

NIPCO Certification, Diplomat Status - Diabetes Care 1997, Respiratory Care 1997, Pharmacist's Care 1998

\section{PROFESSIONAL EXPERIENCE}

Teaching Assistantship, Basic Pharmaceutical Sciences, WVU 1999-present Laboratory manager (supervised and trained new graduate student TAs) for the first year Pharmaceutical Care Laboratory. Taught pre-laboratory and supervised pharmaceutics laboratories on emulsions. Assisted in the Advanced Compounding Laboratory for $3^{\text {rd }}$ year Pharmacy students. Assisted in the Pharmaceutical Care Laboratory for Pharmacy students, graded laboratory reports, and answered questions about the laboratory and laboratory procedures. 
Processed medication orders, answered drug information questions, conducted diabetes and respiratory care evaluations, made recommendations to improve patient drug therapy, used computer for billing purposes, supervised students, conducted in-services for hospital and community.

Program Assistant, Penn State Cooperative Extension

1996-1999

Created and established 'The Tobacco Free Youth Coalition of Monroe County' which is concerned with youth and tobacco issues. Organized annual youth rallies for local high school students, organized compliance checks for local businesses, worked with high school physical education teachers to start programs within their schools, organized a resource center for county residents.

\section{PUBLICATIONS}

Schuler BJ, Aurora TS. Calculation of Defect Formation Energy from the Thermal Expansion Data for Lead Fluoride. Materials Research Society Symposium Procedure, 1994; Volume 369.

\section{POSTER PRESENTATIONS}

BJ Schuler, D Kolwankar, PJ Stout. Formulation of a Sugar Cross-linked Gelatin Matrix for Localized Doxycycline Drug Delivery. American Association for Dental Research - West Virginia Section, Sixth Annual Research Day, Morgantown, WV, February 9, 2000

BJ Schuler, D Kolwankar, PJ Stout. Formulation of a Sugar Cross-linked Gelatin Matrix for Localized Doxycycline Drug Delivery. West Virginia University School of Medicine, Research Day, Morgantown, WV, April 7, 2000

BJ Schuler, D Kolwankar, PJ Stout. Formulation of Sugar Cross-linked Gelatin Matrices for Localized, Sustained Release Drug Delivery. American Association of Pharmaceutical Scientists, Annual Meeting and Exposition, Indianapolis, IN, October 30, 2000

BJ Schuler, PJ Stout. Characterization of Sugar Cross-linked Gelatin Matrices for Localized Drug Delivery. West Virginia University - School of Medicine, Research Day, Morgantown, WV, April 12, 2001

BJ Schuler, PJ Stout. Characterization of Sugar Cross-linked Gelatin Matrices for Localized Drug Delivery. American Association of Pharmaceutical Scientists, Annual Meeting and Exposition, Denver, CO, October 23, 2001

BJ Schuler, PJ Stout. Design of Bioerodable Delivery Systems: Influence of Excipient Selection. West Virginia University - School of Medicine, Research Day, Morgantown, WV, April 12, 2002 
BJ Schuler, PJ Stout. Design of Bioerodable Delivery Systems: Influence of Excipient Selection. American Association of Pharmaceutical Scientists, Annual Meeting and Exposition, Toronto, Ontario, Canada, November 12, 2002

M Sanga, BJ Schuler, SS Wolfe, R Carrier, PS Callery, TM Bland, TS Tracy. Pyrolytic Products of Methamphetamine Hydrochloride as Potential Markers for Smoked Methamphetamine. American Association of Forensic Scientists (AAFS) Annual Meeting, Salt Lake City, UT, March 2003

\section{PROFESSIONAL PRESENTATIONS}

April 15, $1999 \quad$ Better Breather's Club of Carbon County

March 25, 1999 Respiratory Care Society, Lehigh Valley

April 25, 1998 ACLS Provider Care, Palmerton Hospital

April 7, 1998 ACLS Provider Care, Palmerton Hospital

March 31, 1998 ACLS Provider Care, Palmerton Hospital

February 26, 1998 Boy Scouts of America, Pleasant Valley Elementary School

February 24, 1998 Cardiac Rehabilitation Program, Muhlenberg Hospital

October 7,1996 ACLS Provider Care, Palmerton Hospital

August 10, 1995 Cardiac Rehabilitation Program, Muhlenberg Hospital

\section{PROFESSIONAL AFFILIATIONS}

American Association of Pharmaceutical Scientists

American Chemical Society

American Pharmacists Association

American Society of Health-System Pharmacists

Pennsylvania Pharmacist Association 